DOC.20041012.0003

QA: QA

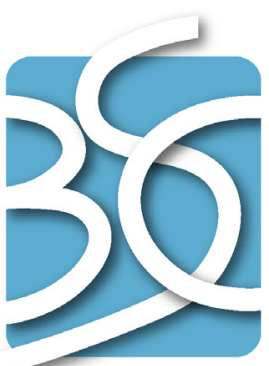

BECHTEL SAIC COMPANYLC

\title{
Aqueous Corrosion Rates for Waste Package
}

Materials

ANL-DSD-MD-000001 REV 01

October 2004

Prepared for:

U.S. Department of Energy

Office of Civilian Radioactive Waste Management

Office of Repository Development

1551 Hillshire Drive

Las Vegas, Nevada 89134-6321

Prepared by:

Bechtel SAIC Company, LLC

1180 Town Center Drive

Las Vegas, Nevada 89144

Under Contract Number

DE-AC28-01RW12101 


\section{DISCLAIMER}

This report was prepared as an account of work sponsored by an agency of the United States Government. Neither the United States Government nor any agency thereof, nor any of their employees, nor any of their contractors, subcontractors or their employees, makes any warranty, express or implied, or assumes any legal liability or responsibility for the accuracy, completeness, or any third party's use or the results of such use of any information, apparatus, product, or process disclosed, or represents that its use would not infringe privately owned rights. Reference herein to any specific commercial product, process, or service by trade name, trademark, manufacturer, or otherwise, does not necessarily constitute or imply its endorsement, recommendation, or favoring by the United States Government or any agency thereof or its contractors or subcontractors. The views and opinions of authors expressed herein do not necessarily state or reflect those of the United States Government or any agency thereof. 
QA: QA

Aqueous Corrosion Rates for Waste Package Materials ANL-DSD-MD-000001 REV 01

October 2004 
2. Scientific Analysis Title

Aqueous Corrosion Rates for Waste Package Materials

3. DI (including Revision Number)

ANL-DSD-MD-000001 REV 01

\begin{tabular}{|c|c|c|c|}
\hline $\begin{array}{l}\text { 4. Total Appendices } \\
\text { Three (3) }\end{array}$ & & $\begin{array}{l}\text { 5. Number of Pages in Each Appendix } \\
\text { I-2; II-2+CD; III-12 }\end{array}$ & \\
\hline & Printed Name & Signature & Date \\
\hline 6. Originator & Sarah Arthur & & \\
\hline 7. Checker & Susan LeStrange & & \\
\hline 8. QER & Darrell Svalstad & & \\
\hline 9. Responsible Manager/Lead & Dennis Thomas & & \\
\hline 10. Responsible Manager & Neil Brown & & \\
\hline
\end{tabular}

11. Remarks

Contributing author: Patricia Bernot

Change History

\begin{tabular}{|l|l|}
\hline \multicolumn{2}{|c|}{ Change History } \\
\hline 12. Revision No. & \multicolumn{1}{c|}{ 13. Description of Change } \\
\hline 00 & Initial Issue. \\
\hline 01 & $\begin{array}{l}\text { Changes to address regulatory integration issues. Corrosion rates of materials } \\
\text { described in other reports (e.g. waste form materials and Alloy 22) are removed. } \\
\text { Corrosion rates for drip shield materials added. Change bars were not used because } \\
\text { changes were too extensive. CR-3489 was addressed by adding the oxygen content of } \\
\text { alloy N06464 to Table 6-2. }\end{array}$ \\
\hline & \multicolumn{2}{|c|}{} \\
\hline & \\
\hline
\end{tabular}




\section{INTENTIONALLY LEFT BLANK}




\section{CONTENTS}

Page

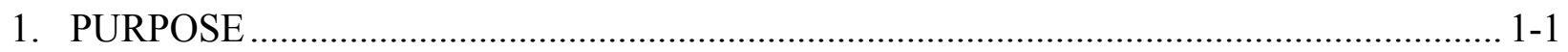

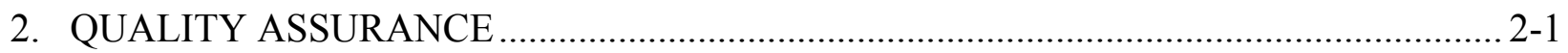

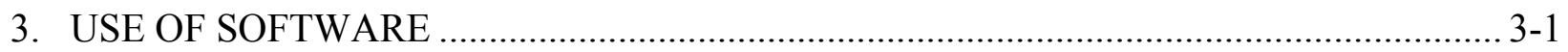

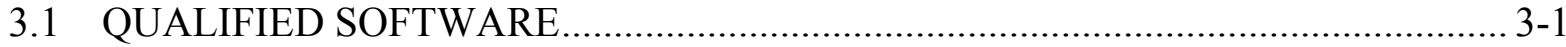

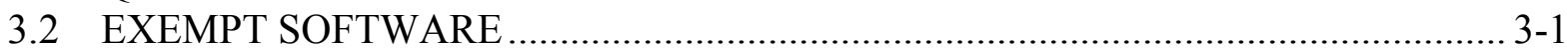

4. INPUTS

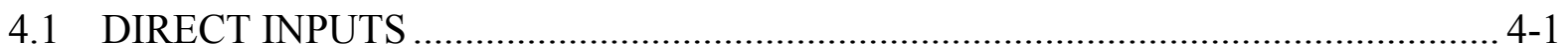

4.1.1 Waste Package Materials ............................................................................ 4-1

4.1.2 Spent Fuels and Waste Forms ................................................................... 4-4

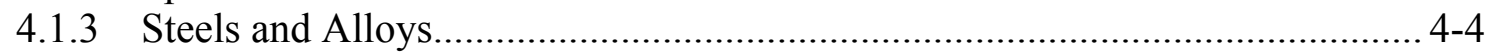

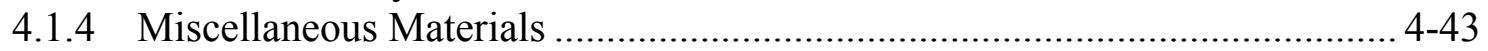

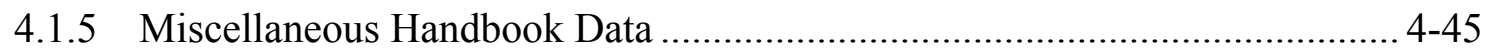

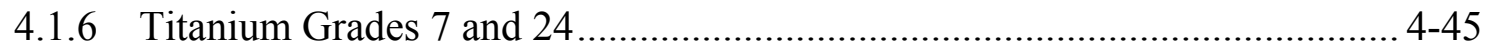

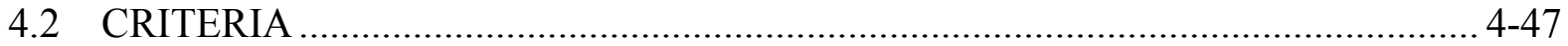

4.3 CODES, STANDARDS, AND REGULATIONS ............................................... 4-47

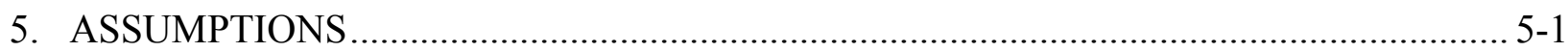

5.1 ASSUMPTIONS CONCERNING STEEL TYPES INVESTIGATED IN THIS

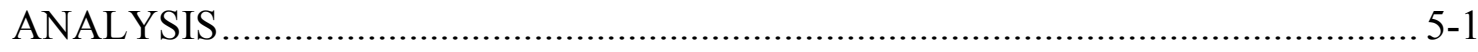

5.1.1 AM-350 Corrosion Rate ……………………........................................ 5-1

5.1.2 Use of Corrosion of Stainless Steel Type 302 ............................................... 5-1

5.2 ASSUMPTIONS CONCERNING ALLOYS INVESTIGATED IN THIS

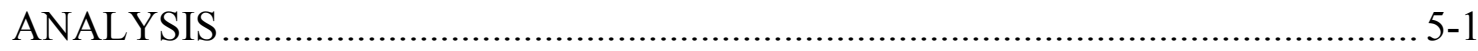

5.2.1 Corrosion of Inconel Alloys 600 and X-750 ............................................ 5-1

5.2.2 Density of Aluminum Alloys.................................................................... 5-2

5.3 ASSUMPTIONS CONCERNING MISCELLANEOUS MATERIALS ..................... 5-2

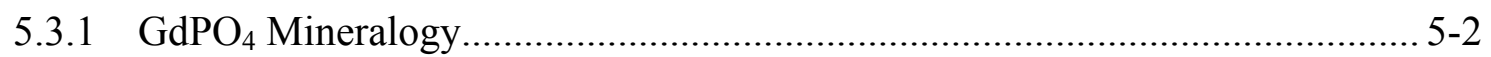

5.4 ASSUMPTIONS CONCERNING GLASS FRACTURING ………………............ 5-3

5.4.1 Cooling Fractures............................................................................ 5-3

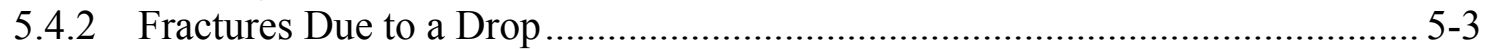

5.4.3 Availability of Cooling Fractures …………….............................................. 5-4

5.4.4 Reactivity of Glass Fractures .................................................................. 5-4

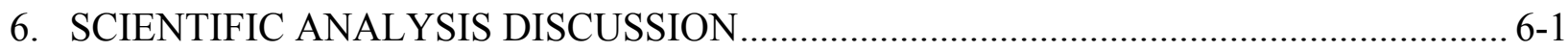

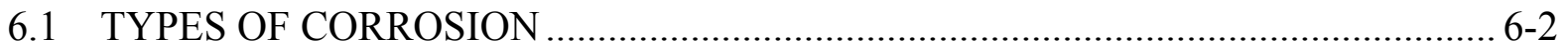

6.1.1 General Corrosion................................................................................. 6-2

6.1.2 Localized Corrosion..................................................................................... 6-2

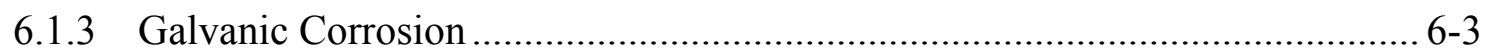

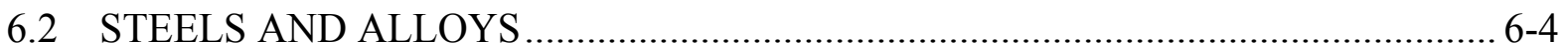

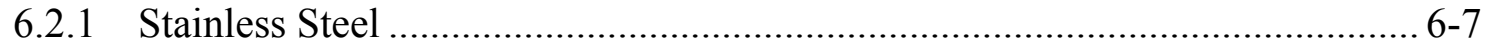

6.2.2 Steel Containing Neutron Absorbers ............................................................ 6-15 


\section{CONTENTS (Continued)}

Page

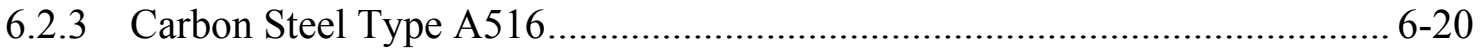

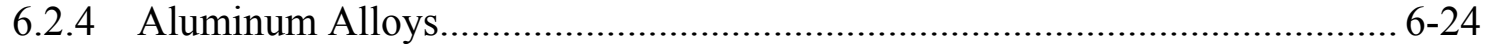

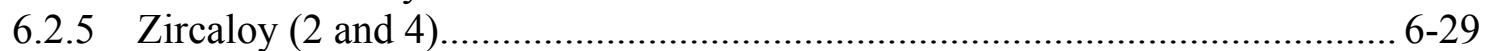

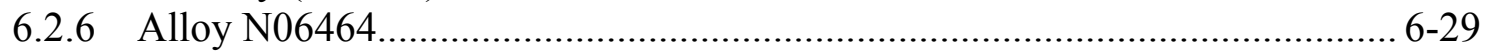

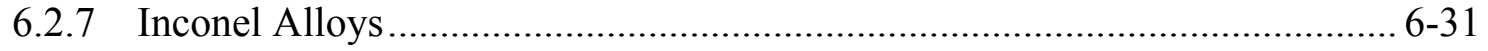

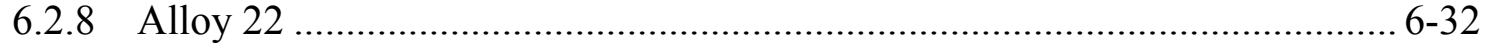

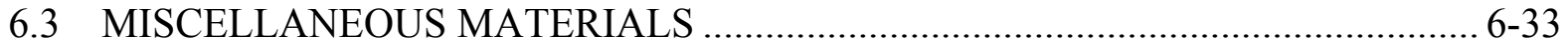

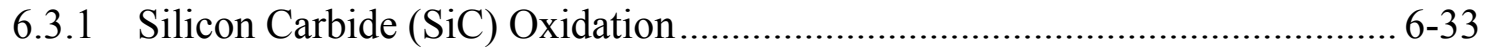

6.3.2 Pyrolytic Carbon and Graphite Oxidation ................................................. 6-34

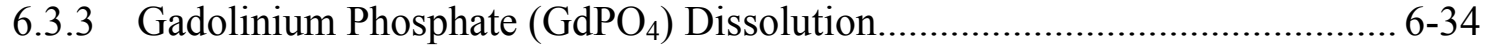

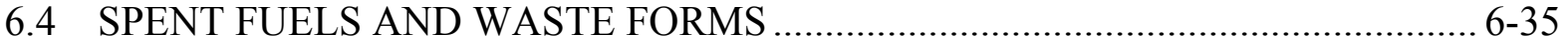

6.4.1 DOE Spent Nuclear Fuel ....................................................................... 6-35

6.4.2 Commercial Spent Nuclear Fuel ........................................................... 6-35

6.4.3 Defense High-Level Waste Glass ............................................................ 6-35

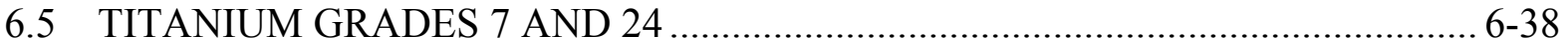

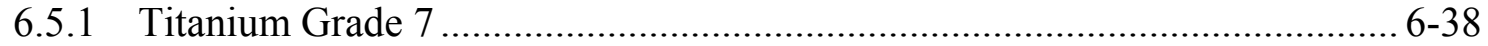

6.5.2 Titanium Grade 24 ................................................................................ 6-39

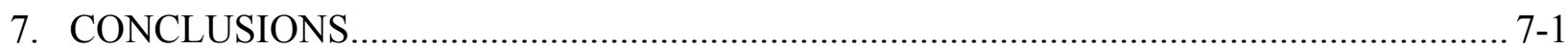

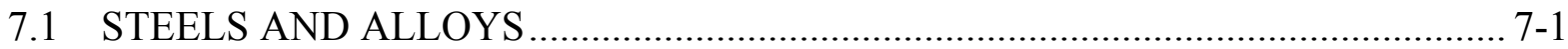

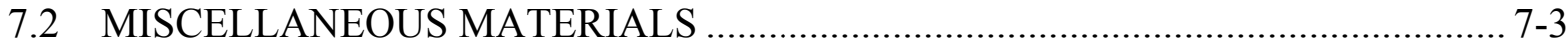

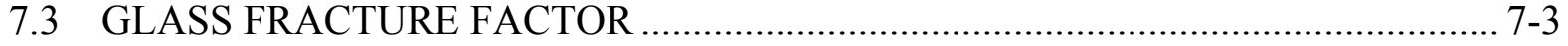

7.4 YUCCA MOUNTAIN REVIEW PLAN ACCEPTANCE CRITERIA ...................... 7-4

8. INPUTS AND REFERENCES ............................................................................ $8-1$

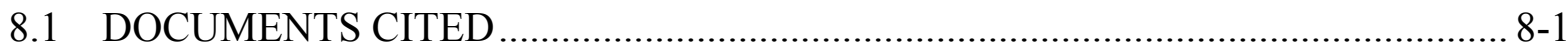

8.2 CODES, STANDARDS, REGULATIONS, AND PROCEDURES ....................... 8-10

8.3 SOURCE DATA, LISTED BY DATA TRACKING NUMBER …...................... 8-12

8.4 OUTPUT DATA, LISTED BY DATA TRACKING NUMBER ............................ 8-12

APPENDIX I $\quad$ LISTING OF FILES ON CD …...........................................................

APPENDIX II ATTACHED CD-ROM ...................................................................... II

APPENDIX III QUALIFICATION OF DATA THAT SUPPORTS AQUEOUS CORROSION RATES FOR WASTE PACKAGES MATERIALS (ANL-DSD-MD-000001)..... 


\section{FIGURES}

6-1. ECDF for Stainless Steel Types 302/304/304L in All Water Types Investigated........... 6-9

6-2. ECDF for Stainless Steel Types 302/304/304L Showing Detail for Slower Rates ......... 6-9

6-3. ECDF for Stainless Steel Types 316/316L/AM-350 in Freshwater ............................ 6-11

6-4. ECDF for Stainless Steel Types 316/316L/AM-350 in Saltwater............................... 6-11

6-5. ECDF for Stainless Steel Types 316/316L/AM-350 in Saltwater Showing More

Detail for Corrosion Rates Below $2.5 \mu \mathrm{m} / \mathrm{yr}$............................................................ 6-12

6-6. Experimental Results of Boil-Down Tests ........................................................ 6-13

6-7. Corrosion Rate of Stainless Steel Type 304L as a Function of $\mathrm{H}_{2} \mathrm{O}_{2}$ from Radiolysis . 6-14

6-8. ECDF for Neutronit (Using Stainless Steel Type 321 Surrogate) in Freshwater ......... 6-16

6-9. ECDF for Neutronit (Using Stainless Steel Type 321 Surrogate) in Saltwater ............ 6-17

6-10. ECDF for 0.3 Percent Borated Stainless Steel Type 304 in All Water Types Investigated ................................................................................................... 6-18

6-11. ECDF for 1.5 Percent Borated Stainless Steel Type 304 in All Water Types

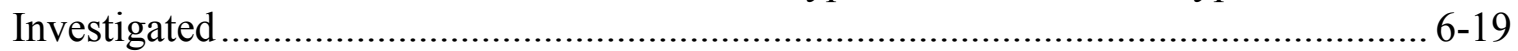

6-12. Corrosion Rates versus Temperature for Mild Steel............................................... 6-22

6-13. ECDF for Carbon Steel Type A516 in All Water Types Investigated (Time $\geq 1$

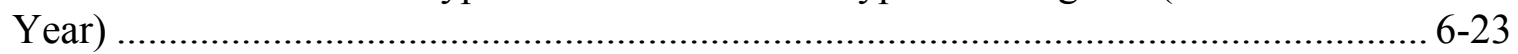

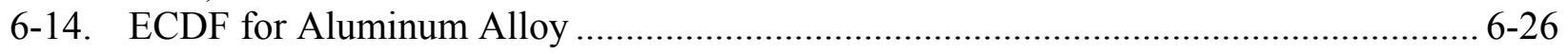

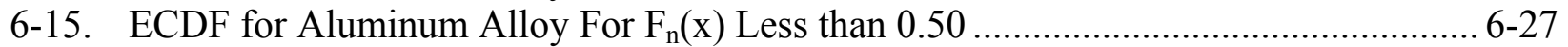

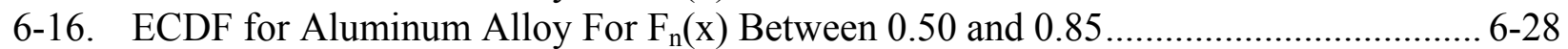

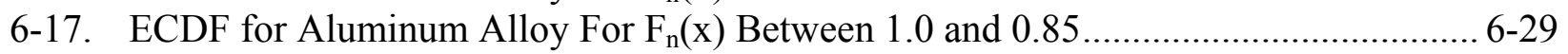

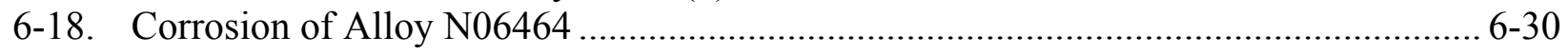

6-19. ECDF for Alloy N06464 in Freshwater at Time $\leq 0.8$ Years .................................... 6-31

6-20. ECDF for Inconel Alloys in All Water Types Investigated..................................... 6-32

6-21. Titanium Grade 7 Weight-Loss Corrosion Rates after 2.5 Years ............................... 6-39

6-22. Corrosion Rates of Titanium Grades 2, 5, 7, 16, and 24 in Boiling HCl.................... 6-40

6-23. ECDF for Titanium Grade 24 in All Water Types Investigated............................... 6-41 


\section{INTENTIONALLY LEFT BLANK}




\section{TABLES}

Page

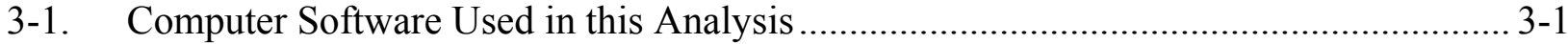

3-2. Computers Used in Analysis............................................................................ 3-1

4-1. $\quad$ Summary of Waste Package Materials .................................................................... 4-3

4-2. Stainless Steel Types 302/304/304L Corrosion in Freshwater ...................................... 4-6

4-3. Stainless Steel Types 316/316L Corrosion in Freshwater ........................................ 4-10

4-4. Stainless Steel Types 302/304/304L Corrosion in Seawater ...................................... 4-12

4-5. Stainless Steel Types 316/316L Corrosion in Seawater ............................................ 4-15

4-6. Corrosion of Stainless Steel Type 304L in Boil-Down Tests.................................... 4-17

4-7. Corrosion Rates of Stainless Steel Type 304L in Solution Containing Radiolysis

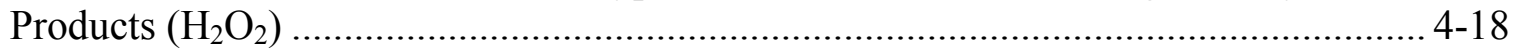

4-8. Neutronit-Corrosion Rates (Using Stainless Steel Type 321 Surrogate) in Freshwater......................................................................................... 4-19

4-9. Neutronit Corrosion Rates (Using Stainless Steel Type 321 Surrogate) in Seawater ... 4-20

4-10. Corrosion of Borated Stainless Steel Type 304L for Use as "Freshwater" Rates ........ 4-21

4-11. Corrosion of Borated Stainless Steel Type 304L for Use as "Saltwater" Rates ........... 4-22

4-12. Corrosion Rates of Gadolinium-Alloyed Steel ........................................................ 4-25

4-13. Carbon Steel Corrosion at 1 Year in Freshwater at $90^{\circ} \mathrm{C}$.......................................... 4-26

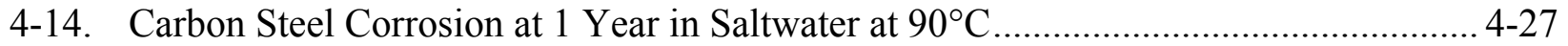

4-15. Carbon Steel Corrosion at 1 Year in Freshwater at $60^{\circ} \mathrm{C} \mathrm{.........................................} \mathrm{4-28}$

4-16. Carbon Steel Corrosion at 1 Year in Saltwater at $60^{\circ} \mathrm{C}$............................................. 4-29

4-17. Carbon Steel Corrosion at 0.53 Years in Freshwater at $90^{\circ} \mathrm{C}$.................................... 4-30

4-18. Carbon Steel Corrosion at 0.5 Years in Saltwater at $90^{\circ} \mathrm{C}$........................................ 4-31

4-19. Carbon Steel Corrosion at 0.52 Years in Freshwater at $60^{\circ} \mathrm{C}$.................................... 4-32

4-20. Carbon Steel Corrosion at 0.50 Years in Saltwater at $60^{\circ} \mathrm{C}$...................................... 4-33

4-21. Corrosion Rates of Aluminum in Seawater ............................................................ 4-34

4-22. Corrosion Rates of Aluminum in Freshwater ........................................................ 4-37

4-23. Corrosion Rates of Alloy N06464 in J-13 Water..................................................... 4-39

4-24. Corrosion Rates of Inconel Alloys in Freshwater .................................................. 4-40

4-25. Corrosion Rates of Inconel Alloys in Acidified Water............................................. 4-40

4-26. Corrosion Rates of Inconel Alloys in Saltwater .................................................... 4-42

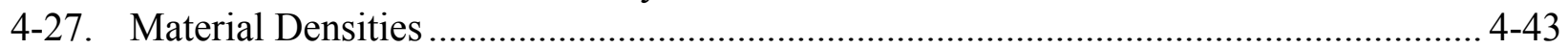

4-28. Corrosion Behavior of Sintered Carbon Materials .................................................... 4-44

4-29. High Temperature Values for SiC Oxidation ..................................................... 4-44

4-30. Miscellaneous Constants Used in Conversions ...................................................... 4-45

4-31. Titanium Grade 7 Weight-Loss Corrosion Rates in Aqueous Media (2.5-Year Data).. 4-46

6-1. Major Bi-Metallic Couples Located Within Waste Packages .................................... 6-4

6-2. Composition (wt \%) of Steel and Alloys .................................................................. 6-5

6-3. Halide Content of Several Waters from Yucca Mountain Strata Compared to J-13

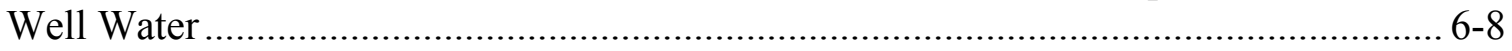

6-4. Minimum, Maximum, Mean, and Median Aqueous Corrosion Rates of Stainless Steel Types 302/304/304L 
6-5. Minimum, Maximum, Mean, and Median Aqueous Corrosion Rates of Stainless Steel Types 316/316L/AM-350

6-6. Minimum, Maximum, Mean, and Median Aqueous Corrosion Rates of Neutronit (Using Stainless Steel Type 321 Surrogate)

6-7. Minimum, Maximum, Mean, and Median Aqueous Corrosion Rates of Borated Stainless Steel Type 304

6-8. Comparison of Minimum, Maximum, Mean, and Median Corrosion Rates of Gadolinium-Alloyed and Non Gadolinium-Alloyed Stainless Steel Type 304L

6-9. Minimum, Maximum, Mean, and Median Aqueous Corrosion Rates of Carbon Steel Type A516

6-10. Pitting in Aluminum Alloys

6-11. Minimum, Maximum, Mean, and Median Aqueous Corrosion Rates of Aluminum Alloy 6-28

6-12. Minimum, Maximum, Mean, and Median Corrosion Rates of Alloy N06464 in Solution

6-13. Minimum, Maximum, Mean, and Median Aqueous Corrosion Rates of Inconel Alloys..... $6-32$

6-14. Approximate Values of Oxidation for Graphite and Pyrolytic Carbon 6-34

6-15. Probability of Loading Damaged Glass Pour Canisters into Waste Packages. 6-36

6-16. Fracture Factor Probabilities (Fracture Factor Averaged Over 5 Glass Pour Canisters) for High Level Waste Glass Based on Loading Damaged Glass Pour Canisters into Waste Packages.

6-17. True Fracture Factors for High Level Waste Glass in One Glass Pour Canister. 6-38

6-18. Minimum, Maximum, Mean, and Median Corrosion Rates of Titanium Alloy Grade 24 in Solution

7-1. Summary of Statistical Values of Corrosion for Waste Package Metals. 7-1

7-2. Comparison of Minimum, Maximum, Mean, and Median Corrosion Rates of Gadolinium-Alloyed and Non Gadolinium-Alloyed Stainless Steel Type 304.

7-3. Summary of Corrosion Behavior and Rates for Graphite, SiC, and Gadolinium Phosphate

7-4. Fracture Factor Probabilities (Fracture Factor Averaged Over 5 Glass Pour Canisters) for High-Level Waste Glass Based on Loading Damaged Glass Pour Canisters into Waste Packages.

7-5. True Fracture Factors for High-Level Waste Glass in One Damaged Glass Pour Canister 


\section{ACRONYMS AND ABBREVIATIONS}

ANL

ATWT

BSC

BWR

CDF

CRWMS

CSNF

DHLW

DOE

DTN

ECDF

EPRI

FFTF

FSVR

LA

LWBR

LLNL

LTCTF

$\mu \mathrm{m}$

mil

$\mathrm{M} \& \mathrm{O}$

$\mathrm{MCO}$

MOX

MR

PNNL

PWR

SAW

SCW

SDW

SKB

SNF

TRIGA

TSPA

TSPA-LA
Argonne National Laboratory

atomic weight

Bechtel SAIC Company

boiling water reactor

cumulative distribution function

Civilian Radioactive Waste Management System

commercial spent nuclear fuel

defense high-level waste

U.S. Department of Energy

data tracking number

empirical cumulative distribution function

Electric Power Research Institute

Fast Flux Test Facility

Fort Saint Vrain Reactor

License Application

light water breeder reactor

Lawrence Livermore National Laboratory

Long Term Corrosion Test Facility

micron or micrometer, or $10^{-6}$ meter

$1 / 1,000^{\text {th }}$ of an inch, or $25.4 \mu \mathrm{m}$

Management and Operating Contractor

multi-canister overpack

mixed oxide

model report

Pacific Northwest National Laboratory

pressurized water reactor

simulated acidified well

simulated concentrated well

simulated dilute well

Swedish Nuclear Fuel and Waste Management Company

spent nuclear fuel

Training, Research, Isotopes, General Atomics

Total System Performance Assessment

Total System Performance Assessment License Application 


\section{INTENTIONALLY LEFT BLANK}




\section{PURPOSE}

The purpose of this analysis, as directed by Technical Work Plan for: Regulatory Integration Modeling and Analysis of the Waste Form and Waste Package (BSC 2004 [DIRS 171583]), is to compile applicable corrosion data from the literature (journal articles, engineering documents, materials handbooks, or standards, and national laboratory reports), evaluate the quality of these data, and use these to perform statistical analyses and distributions for aqueous corrosion rates of waste package materials. The purpose of this report is not to describe the performance of engineered barriers for the TSPA-LA. Instead, the analysis provides simple statistics on aqueous corrosion rates of steels and alloys.

These rates are limited by various aqueous parameters such as temperature (up to $100^{\circ} \mathrm{C}$ ), water type (i.e., fresh versus saline), and $\mathrm{pH}$. Corrosion data of materials at $\mathrm{pH}$ extremes (below 4 and above 9) are not included in this analysis, as materials commonly display different corrosion behaviors under these conditions. The exception is highly corrosion-resistant materials (Inconel Alloys) for which rate data from corrosion tests at a $\mathrm{pH}$ of approximately 3 were included. The waste package materials investigated are those from the long and short 5-DHLW waste packages, 2-MCO/2-DHLW waste package, and the 21-PWR commercial waste package. This analysis also contains rate data for some of the materials present inside the fuel canisters for the following fuel types: U-Mo (Fermi U-10\%Mo), MOX (FFTF), Thorium Carbide and Th/U Carbide (Fort Saint Vrain [FSVR]), Th/U Oxide (Shippingport LWBR), U-metal (N Reactor), Intact U-Oxide (Shippingport PWR, Commercial), aluminum-based, and U-Zr-H (TRIGA).

Analysis of corrosion rates for Alloy 22, spent nuclear fuel, defense high level waste (DHLW) glass, and Titanium Grade 7 can be found in other analysis or model reports (See Sections 6.2.8, 6.4, and 6.5.1).

This analysis supports risk and criticality calculations or analyses, but will not be used directly in the TSPA-LA. Output from this analysis will be used to support calculations or analyses using the EQ6 computer code for reaction-path modeling of aqueous geochemical systems (Wolery and Daveler 1992 [DIRS 100097]). The following downstream criticality documents use this analysis or its output data as input: Impacts of Updated Design and Rates on EQ6 Calculations for Chemical Degradation of Fermi and TRIGA Codisposal Waste Packages, EQ6 Calculations for Chemical Degradation of ATR Aluminum-Based DOE SNF in a Waste Package, Screening Analysis of Criticality Features, Events, and Processes for License Application, Boron Loss from CSNF Waste Packages, Probability of External Criticality for Codisposal Waste Packages, and Configuration Generator Model for In-Package Criticality. This analysis supports the determination of the probability of criticality for DOE SNF codisposal waste packages by providing, where appropriate: 1) minimum, maximum, mean, and median values of corrosion rates with the corresponding standard deviations; and 2) empirical cumulative distribution functions (ECDFs) of the corrosion rates of materials.

Output from this report is limited to general corrosion rates of waste package materials. A statistical analysis of galvanic and localized corrosion rates is not provided in this analysis. 


\section{INTENTIONALLY LEFT BLANK}




\section{QUALITY ASSURANCE}

The preparation of this analysis and the supporting activities are subject to Quality Assurance Requirements and Description (DOE 2004 [DIRS 171539]), as stated in the technical work plan (BSC 2004 [DIRS 171583]). This analysis was prepared in accordance with AP-SIII.9Q, Technical Reports, and reviewed in accordance AP-2.14Q, Document Review. It does not investigate any items in Q-List (BSC 2004 [DIRS 168361]).

The control of the electronic management of information was evaluated in accordance with AP-SV.1Q, Control of the Electronic Management of Information. Methods used for electronic information control, as specified in the technical work plan (BSC 2004 [DIRS 171583]) include:

- Records submitted in accordance with AP-17.1Q, Records Management, and AP-6.1Q, Document Control, are retrievable through the records processing center.

- Engineering technical information stored on writeable CD-ROMs that are not rewriteable. Conditions, location, retention time, and access are controlled through the records center following submittal in accordance with AP-17.1Q, Records Management, and AP-6.1Q, Document Control.

- Media identified in accordance with AP-17.1Q, Records Management, and AP-SIII.9Q, Technical Reports. 


\section{INTENTIONALLY LEFT BLANK}




\section{USE OF SOFTWARE}

\subsection{QUALIFIED SOFTWARE}

No software that requires project or application-specific qualification or validation in accordance with LP-SI.11Q-BSC, Software Management, was used in this analysis.

\subsection{EXEMPT SOFTWARE}

This section describes the computer software used to carry out the calculations in Section 4 and the regression analysis in Section 6. Table 3-1 lists computer software used in this analysis.

Table 3-1. Computer Software Used in this Analysis

\begin{tabular}{|l|l|l|l|l|}
\hline $\begin{array}{c}\text { Software } \\
\text { Name }\end{array}$ & \multicolumn{1}{|c|}{ Version } & \multicolumn{1}{|c|}{$\begin{array}{c}\text { Software Tracking } \\
\text { Number } \\
\text { (Qualification Status) }\end{array}$} & \multicolumn{1}{c|}{$\begin{array}{c}\text { Description and } \\
\text { Components Used }\end{array}$} & $\begin{array}{c}\text { Input and Output } \\
\text { Files (Included in } \\
\text { Appendix II) }\end{array}$ \\
\hline $\begin{array}{l}\text { Microsoft } \\
\text { Excel }\end{array}$ & $\begin{array}{l}\text { Versions } 97 \\
\text { SR-2, 9.0.4402 } \\
\text { SR-1 }\end{array}$ & $\begin{array}{l}\text { Commercial off-the- } \\
\text { shelf software }\end{array}$ & $\begin{array}{l}\text { Used in this document for } \\
\text { graphical representation and } \\
\text { arithmetical manipulations }\end{array}$ & output: * *xls \\
\hline
\end{tabular}

Microsoft Excel for Windows 97 (Versions SR-1 and SR-2) is used in calculations and analyses to manipulate the inputs using standard mathematical expressions and operations. It is also used to tabulate and chart results. The user-defined formulas, inputs, and results are documented in sufficient detail to allow an independent repetition of computations. Thus, Microsoft Excel is used only as a worksheet, not as a software routine; therefore, in accordance with LP-SI.11Q-BSC, Software Management, it is not required to be qualified or documented.

Microsoft Excel was run on standard personal computers listed in Table 3-2.

Table 3-2. Computers Used in Analysis

\begin{tabular}{|l|l|l|l|}
\hline \multicolumn{1}{|c|}{ Computer Make } & \multicolumn{1}{c|}{ CPU \# } & \multicolumn{1}{c|}{$\begin{array}{c}\text { Operating } \\
\text { System }\end{array}$} & \multicolumn{1}{c|}{ Software Used } \\
\hline Dell Optiplex GX300 & CRWMS-M\&O Tag \# 117728 & Windows 95 & Microsoft Excel (97 SR-2) \\
\hline Dell Latititude C/Port II APR & $\begin{array}{l}\text { Framatome ANP Inc. Service } \\
\text { Tag \# H22MT11 }\end{array}$ & Windows 2000 & Microsoft Excel (9.0.4402 SR-1) \\
\hline Dell Optiplex GX260 & CRWMS-M\&O Tag \# 152393 & Windows 2000 & Microsoft Excel (97 SR-2) \\
\hline
\end{tabular}




\section{INTENTIONALLY LEFT BLANK}




\section{INPUTS}

\subsection{DIRECT INPUTS}

This section includes the types of materials used within the waste packages, as well as the corrosion rate data of those materials. Section 4.1.1 lists the components of waste packages analyzed in this report and identifies the materials of which these are made. Input data and information necessary to perform the analyses for these materials are provided in Sections 4.1.2 through 4.1.5. Section 4.1.6 includes input data and information for drip shield materials.

\subsubsection{Waste Package Materials}

There are four basic waste package designs used in geochemistry calculations for DOE and commercial SNF. These include 5-DHLW long and short, 2-MCO/2-DHLW, and commercial 21-PWR waste packages. The major components of these waste package types and the materials of which these are made are provided in the following sections. A synopsis of waste package materials is presented in Table 4-1.

\subsubsection{Components Common to All DOE Waste Packages}

The following components are common to all DOE waste packages:

- The outer shell, constructed of Alloy 22 (BSC 2004 [DIRS 169472])

- The inner vessel (also called liner) constructed of Stainless Steel Type 316 (BSC 2004 [DIRS 169472])

- A Carbon Steel Type A516 structural basket (divider plate assembly or fuel divider assembly). For 5-DHLW packages (BSC 2004 [DIRS 166860]; BSC 2004 [DIRS 166861]) per D\&E / PA/C IED Typical Waste Package Components Assembly (BSC 2004 [DIRS 169480]), the structural basket is designed to hold the high-level waste glass pour canisters in place. For the 2-MCO/2-DHLW packages (BSC 2004 [DIRS 166862]) per D\&E/PA/C IED Typical Waste Package Components Assembly (BSC 2004 [DIRS 169480]), the basket consists of perpendicularly aligned A-plates designed to hold the glass pour canisters and multicanister overpacks (MCOs) in place.

- The glass pour canisters, consisting of Stainless Steel Type 304L canisters (Baxter 1988 [DIRS 106164]), are filled with solidified high-level waste glass. 


\subsubsection{Components of the 5-DHLW Long and Short Waste Packages}

The major fuel types that will potentially be stored in these waste packages include Shippingport PWR, Shippingport LWBR, Fort Saint Vrain, FFTF, aluminum-based, Fermi, and TRIGA. It is convenient to consider the 5-DHLW waste packages as several structural components:

- Centrally located Stainless Steel Type 316 DOE SNF canister (sometimes called the "18-inch canister") with internal Carbon Steel Type A516 impact plates (DOE 1999 [DIRS 140225]).

- Individual DOE SNF canister internals, including:

- Shippingport PWR - borated Stainless Steel Type 304L, chrome plating, Inconel X spacer rings, Zircaloy-4 fuel cluster structure, Zircaloy-2 spacers, and $\mathrm{UO}_{2}-\mathrm{CaO}-\mathrm{ZrO}_{2}$ alloy fuel wafers (DOE 1999 [DIRS 104940])

- Shippingport LWBR - Stainless Steel Type 316L basket and spacers, AM-350 stainless steel grid, Aluminum Alloy Type 6061-T6 fill material with 1\% added gadolinium, Zircaloy-4 cladding and support shell, Inconel X-750 Wire Compression Spring, Inconel 600 base plates, $\mathrm{ThO}_{2}$, and $\mathrm{UO}_{2}-\mathrm{ThO}_{2}$ SNF (DOE 1999 [DIRS 105007])

- Fort Saint Vrain - fuel elements (graphite blocks that hold the fuel compacts). The fuel compacts contain the fuel kernels. Fuel kernels are composed of a Thorium Carbide or $\mathrm{Th} / \mathrm{U}$ carbide core surrounded by four protective coatings of $\mathrm{SiC}$ and pyrolytic carbon (Taylor 2001 [DIRS 154726])

- FFTF - (“driver assembly") Stainless Steel Type 316 cladding, wire, and spacers, Inconel 600 reflectors, Stainless Steel Type 316L pins, Stainless Steel Type 316L basket doped with $2.75 \mathrm{wt} \% \mathrm{GdPO}_{4}$, Stainless Steel Type 302 spring, $\mathrm{MOX}\left(\mathrm{UO}_{2}\right.$ and $\mathrm{PuO}_{2}$ ) fuel, and $\mathrm{UO}_{2}$ fuel (INEEL 2002 [DIRS 158820])

- Aluminum-Based Fuels - Fuel assemblies composed of aluminum-based fuels, Aluminum Alloy Types 1100 or 6061-T6 cladding and frame, Aluminum Alloy Type 6061-T6 side plates, comb, and pin (Paige 1969 [DIRS 167978]). Basket composed of Alloy N06464 and Stainless Steel Type 304L

- Enrico-Fermi (Fermi) - Stainless Steel Type 316L basket, Carbon Steel Type A516 and $\mathrm{GdPO}_{4}$ shot (filler material), U-Mo fuel meat metallurgically bonded to a zirconium cladding tube (DOE 1999 [DIRS 104110])

- TRIGA - Graphite reflectors, Zr rods, Stainless Steel Type 304L cladding, and U-Zr hydride fuel (DOE 1999 [DIRS 103891]).

\subsubsection{Components of the 2-MCO/2-DHLW Waste Package}

The following components are found in the 2-MCO/2-DHLW waste packages: 
- Two MCO stands composed of Carbon Steel Type A516 (DOE 2000 [DIRS 150095])

- Two Stainless Steel Type 304L MCOs (DOE 2000 [DIRS 150095])

- MCO internals - Stainless Steel Type 304L baskets, aluminum (Aluminum Alloy Type 1100) spacers, N Reactor (U-metal) SNF rods (DOE 2000 [DIRS 150095]).

\subsubsection{CSNF Waste Package}

The CSNF waste package also contains the Alloy 22 outer shell and Stainless Steel Type 316 inner vessel. The fuel basket assembly, which is located inside the Stainless Steel Type 316 liner, is made up of the following components:

- Four Fuel Plate Assemblies constructed of plates of Alloy N06464 and Aluminum Alloy Type 6061 (BSC 2004 [DIRS 171319]; BSC 2004 [DIRS 171320]) (former design used Neutronit, BSC 2004 [DIRS 169472])

- Four Side/Corner-Guide/Stiffeners constructed of Carbon Steel Type A516 (BSC 2004 [DIRS 169472]

- 21 Fuel Basket Tubes constructed of Carbon Steel Type A516 (BSC 2004 [DIRS 169472])

- 21-PWR Fuel Assemblies constructed of zircaloy cladding and PWR fuel (BSC 2003 [DIRS 169110]; Punatar 2001 [DIRS 155635]).

Table 4-1. Summary of Waste Package Materials

\begin{tabular}{|c|c|c|c|}
\hline $\begin{array}{c}\text { Fuel Types/Waste } \\
\text { Forms }^{\text {a }}\end{array}$ & Steels & Alloys & $\begin{array}{c}\text { Miscellaneous } \\
\text { Materials }\end{array}$ \\
\hline U-metal & Stainless Steel Type 302 & Zircaloy-2 & $\mathrm{GdPO}_{4}$ \\
\hline U-Mo & Stainless Steel Type 304L & Zircaloy-4 & $\mathrm{SiC}$ \\
\hline Aluminum-Based SNF & Stainless Steel Types 316/316L & Alloy $22^{c}$ & Graphite \\
\hline MOX & Carbon Steel Type A516 & Aluminum Alloy Type 1100 & \\
\hline $\mathrm{UO}_{2}$ & Stainless Steel Type AM-350 & Aluminum Alloy Type 6061-T6 & \\
\hline U-Th/Th Oxide & Gd-Doped Stainless Steel Type & Gd-doped Aluminum Alloy Type & \\
\hline U-Th/Th Carbide & $316 L^{\circ}$ & $6061^{\circ}$ & \\
\hline Ca-U-Zr Oxide & Borated Stainless Steel Type 304L & Inconel X-750 & \\
\hline U-Zr Hydride & Neutronit & Inconel 600 & \\
\hline Commercial $\cup_{2}$ & & Alloy N06464 & \\
\hline DHLW Glass & & Chrome plating $^{\mathrm{d}}$ & \\
\hline
\end{tabular}

NOTES: ${ }^{a}$ See Section 4.1.2.

${ }^{\mathrm{b}}$ No rate data was available for these materials and they are not analyzed further in this document.

${ }^{c}$ See Section 4.1.3.6.

${ }^{\mathrm{d}}$ See Section 4.1.3.9.

This table summarizes Sections 4.1.1.1 through 4.1.1.4. 


\subsubsection{Drip Shield Material}

The drip shield is not a waste package component. However, its proximity and position relative to the waste package warrants investigation of corrosion rates for drip shield materials. According to D\&E / PA/C IED Interlocking Drip Shield and Emplacement Pallet (BSC 2004 [DIRS 169220], the primary components of the drip shield are composed of Titanium Grades 7 (SB-265 R52400) and 24 (SB-265 R56405).

\subsubsection{Spent Fuels and Waste Forms}

Since analysis of degradation and dissolution rates for DOE fuels and waste forms, including high-level waste glass and commercial SNF, can be found in other sources (Section 6.4), no further analysis of this information is provided in this document.

\subsubsection{Steels and Alloys}

\subsubsection{Stainless Steel (Types 302/304/304L and 316/316L/AM-350)}

The corrosion rates for different types of stainless steel are presented in Sections 4.1.3.1.1 through 4.1.3.1.5. These sections are divided into various environmental conditions to correlate with Section 6.2 as properties of the aqueous solution determine the rate of corrosion for stainless steels (Section 6.2). Discussions of corrosion rates for Stainless Steel Type 304L also apply to Stainless Steel Types 304 and 302 (Section 5.1.2). Discussions of corrosion rates for Stainless Steel Type 316L also apply to Stainless Steel Types 316 and AM-350 (Section 5.1.1).

For this analysis, all values for corrosion of stainless steels were converted to the same units of $\mu \mathrm{m} / \mathrm{yr}$. When only a value in $\mu \mathrm{m} / \mathrm{yr}$ is provided in tables, the rates were already in those units in the reference. When values for the corrosion rate are presented in any other units in the tables, these are the units presented in the reference, which are then converted to a rate in $\mu \mathrm{m} / \mathrm{yr}$ and indicated with an asterisk. These converted rates are calculated in spreadsheets in Appendix II. These calculated rates are presented in Section 4 along with the rates from the original source for transparency and tracebility. When a value of zero corrosion was reported in the reference, the detection limit of the measurement equipment was used (and recorded in the tables) as the weight loss.

\subsection{Corrosion of Stainless Steel in Fresh/Dilute Waters}

Corrosion rates for Stainless Steel Types 304L and 316L, used in this analysis for fresh or dilute waters (similar to J-13 well water, DTN: MO0006J13WTRCM.000 [DIRS 151029]), can be found in Table 4-2 and Table 4-3. Further explanation on the use of these rates can be found in Sections 6.2.1.1 and 6.2.1.2.

\subsection{Corrosion of Stainless Steel in Saltwater}

General corrosion rates for Stainless Steel Types 304L and 316L in saltwater are found in Table 4-4 and Table 4-5. Further explanation on the use of these rates can be found in Sections 6.2.1.1 and 6.2.1.2. 


\subsection{Boil-Down Tests}

The results from 80-week boil-down tests are located in Table 4-6. Section 6.2.1.3 provides further explanation on the use of these rates.

\subsection{Radiolysis}

The results of experiments simulating the effects of radiolysis products on corrosion can be found in Table 4-7. Section 6.2.1.4 further explains the use of these rates.

\subsection{Sensitization of Stainless Steel}

This section does not report any data on corrosion of sensitized stainless steel. The rationale for this can be found in Section 6.2.1.5.

\subsubsection{Steels Containing Neutron Absorbers (Boron and Gadolinium)}

Neutronit corrosion rates are based on the corrosion of Stainless Steel Type 321 (Section 6.2.2.1) and can be found in Tables 4-8 and 4-9. The corrosion rates of borated Stainless Steel Type 304L and gadolinium-alloyed stainless steel are also presented below in Table 4-10 through Table 4-12. Further explanation on the use of these rates can be found in Sections 6.2.2.2 and 6.2.2.3. All rates for corrosion of stainless steel were converted to the same units of $\mu \mathrm{m} / \mathrm{yr}$. These converted rates are presented in this section along with their values in the original units taken directly from the sources. These converted rates are determined in spreadsheets in Appendix II. 
Table 4-2. Stainless Steel Types 302/304/304L Corrosion in Freshwater

\begin{tabular}{|c|c|c|c|c|c|c|c|c|c|}
\hline $\begin{array}{l}\text { See } \\
\text { Note }\end{array}$ & $\begin{array}{c}\text { Time } \\
\text { (years) }\end{array}$ & $\begin{array}{c}\text { Corrosion } \\
\text { Rate }\end{array}$ & $\begin{array}{l}\text { Corrosion } \\
\text { Rate }(\mu \mathrm{m} / \mathrm{yr})\end{array}$ & $\begin{array}{l}\text { Temp } \\
\left({ }^{\circ} \mathrm{C}\right)\end{array}$ & $\begin{array}{c}\text { Type of } \\
\text { Analysis }\end{array}$ & $\begin{array}{l}\text { Steel } \\
\text { Type }\end{array}$ & $\begin{array}{l}\text { Table in } \\
\text { Source }\end{array}$ & Fluid Type & Other Comments \\
\hline a & 1.00 & $\mathrm{~N} / \mathrm{A}$ & 0.0811 & 28 & WL & $304 \mathrm{~L}$ & 7 & $\begin{array}{l}\text { Irradiated J-13 Well Water } \\
\text { Containing Crushed Tuff }\end{array}$ & $\begin{array}{l}\text { Average of } 6 \text { Stainless Steel Type } \\
304 \mathrm{~L} \text { Coupons in Set }\end{array}$ \\
\hline a & 1.00 & N/A & 0.151 & 28 & WL & $304 \mathrm{~L}$ & 7 & Irradiated J-13 Well Water & $\begin{array}{l}\text { Average of } 6 \text { Stainless Steel Type } \\
\text { 304L Coupons in Set }\end{array}$ \\
\hline a & 1.00 & N/A & 0.123 & 28 & WL & $304 \mathrm{~L}$ & 7 & $\begin{array}{l}\text { Irradiated J-13 Well Water } \\
\text { Containing Crushed Tuff }\end{array}$ & $\begin{array}{l}\text { Average of } 6 \text { Stainless Steel Type } \\
304 \mathrm{~L} \text { Coupons in Set, Heat-Treated, } \\
650^{\circ} \mathrm{C} \text { for } 1 \text { Hour }\end{array}$ \\
\hline a & 1.00 & N/A & 0.116 & 28 & WL & $304 \mathrm{~L}$ & 7 & Irradiated J-13 Well Water & $\begin{array}{l}\text { Average of } 6 \text { Stainless Steel Type } \\
304 \mathrm{~L} \text { Coupons in Set, Heat-Treated, } \\
650^{\circ} \mathrm{C} \text { for } 1 \text { Hour }\end{array}$ \\
\hline a & 1.00 & $\mathrm{~N} / \mathrm{A}$ & 0.242 & 28 & WL & $304 \mathrm{~L}$ & 7 & $\begin{array}{l}\text { Nonirradiated J-13 Well } \\
\text { Water Containing Crushed } \\
\text { Tuff }\end{array}$ & $\begin{array}{l}\text { Average of } 6 \text { Stainless Steel Type } \\
\text { 304L Coupons in Set }\end{array}$ \\
\hline a & 1.00 & N/A & 0.285 & 28 & WL & $304 \mathrm{~L}$ & 7 & $\begin{array}{l}\text { Nonirradiated J-13 Well } \\
\text { Water }\end{array}$ & $\begin{array}{l}\text { Average of } 6 \text { Stainless Steel Type } \\
304 \mathrm{~L} \text { Coupons in Set }\end{array}$ \\
\hline a & 1.00 & N/A & 0.249 & 28 & WL & $304 \mathrm{~L}$ & 7 & $\begin{array}{l}\text { Nonirradiated J-13 Well } \\
\text { Water Containing Crushed } \\
\text { Tuff }\end{array}$ & $\begin{array}{l}\text { Average of } 6 \text { Stainless Steel Type } \\
304 \mathrm{~L} \text { Coupons in Set, Heat-Treated, } \\
650^{\circ} \mathrm{C} \text { for } 1 \text { Hour }\end{array}$ \\
\hline a & 1.00 & $N / A$ & 0.283 & 28 & WL & $304 \mathrm{~L}$ & 7 & $\begin{array}{l}\text { Nonirradiated J-13 Well } \\
\text { Water }\end{array}$ & $\begin{array}{l}\text { Average of } 6 \text { Stainless Steel Type } \\
304 \mathrm{~L} \text { Coupons in Set, Heat-Treated, } \\
650^{\circ} \mathrm{C} \text { for } 1 \text { Hour }\end{array}$ \\
\hline a & 1.31 & $\mathrm{~N} / \mathrm{A}$ & 0.133 & 50 & WL & $304 \mathrm{~L}$ & 6 & Freshwater & Average of 3 Specimens \\
\hline a & 1.26 & $\mathrm{~N} / \mathrm{A}$ & 0.085 & 80 & WL & $304 \mathrm{~L}$ & 6 & Freshwater & Average of 3 Specimens \\
\hline$a$ & 1.18 & $\mathrm{~N} / \mathrm{A}$ & 0.072 & 100 & WL & $304 \mathrm{~L}$ & 6 & Freshwater & Average of 3 Specimens \\
\hline & & $\begin{array}{l}\text { Detection } \\
\text { Limit }(\mathbf{g})^{\mathrm{h}}\end{array}$ & & & & & & & \\
\hline $\mathrm{b}$ & 1 & 0.01 & $0.0113^{*}$ & $29.5^{c}$ & WL & 302 & 2 & Freshwater & $\begin{array}{l}\text { Gatun Lake, Panama Canal Zone, } \\
\text { Immersion Test }\end{array}$ \\
\hline$b$ & 2 & 0.01 & $0.0057^{*}$ & $29.5^{c}$ & WL & 302 & 2 & Freshwater & $\begin{array}{l}\text { Gatun Lake, Panama Canal Zone, } \\
\text { Immersion Test }\end{array}$ \\
\hline $\mathrm{b}$ & 4 & 0.01 & $0.0028^{*}$ & $29.5^{c}$ & WL & 302 & 2 & Freshwater & $\begin{array}{l}\text { Gatun Lake, Panama Canal Zone, } \\
\text { Immersion Test }\end{array}$ \\
\hline $\mathrm{b}$ & 8 & 0.01 & $0.0014^{*}$ & $29.5^{c}$ & WL & 302 & 2 & Freshwater & $\begin{array}{l}\text { Gatun Lake, Panama Canal Zone, } \\
\text { immersion Test }\end{array}$ \\
\hline
\end{tabular}


Table 4-2. Stainless Steel Types 302/304/304L Corrosion in Freshwater (Continued)

\begin{tabular}{|c|c|c|c|c|c|c|c|c|c|}
\hline $\begin{array}{l}\text { See } \\
\text { Note }\end{array}$ & $\begin{array}{c}\text { Time } \\
\text { (years) }\end{array}$ & $\begin{array}{c}\text { Corrosion } \\
\text { Rate }\end{array}$ & $\begin{array}{l}\text { Corrosion } \\
\text { Rate }(\mu \mathrm{m} / \mathrm{yr})\end{array}$ & $\begin{array}{l}\text { Temp } \\
\left({ }^{\circ} \mathrm{C}\right)\end{array}$ & $\begin{array}{c}\text { Type of } \\
\text { Analysis }\end{array}$ & $\begin{array}{l}\text { Steel } \\
\text { Type }\end{array}$ & $\begin{array}{l}\text { Table in } \\
\text { Source }\end{array}$ & Fluid Type & Other Comments \\
\hline$d$ & 16 & 0.01 & $0.0007^{*}$ & $29.5^{c}$ & WL & 302 & 4 & Freshwater & $\begin{array}{l}\text { Gatun Lake, Panama Canal Zone, } \\
\text { Immersion Test }\end{array}$ \\
\hline$d$ & 1 & 0.01 & $0.0478^{*}$ & $29.5^{\mathrm{c}}$ & WL & 302 & 6 & Freshwater & $\begin{array}{l}\text { Gatun Lake, Panama Canal Zone, } \\
\text { Immersion Test, 302/302 Couple }\end{array}$ \\
\hline \multicolumn{10}{|c|}{ mils } \\
\hline$d$ & 8 & 0.1 & $0.3175^{*}$ & $29.5^{\mathrm{c}}$ & WL & 302 & 6 & Freshwater & $\begin{array}{l}\text { Gatun Lake, Panama Canal Zone, } \\
\text { Immersion Test, } 302 / 302 \text { Couple }\end{array}$ \\
\hline$d$ & 16 & 0.1 & $0.1588^{*}$ & $29.5^{\mathrm{c}}$ & WL & 302 & 6 & Freshwater & $\begin{array}{l}\text { Gatun Lake, Panama Canal Zone, } \\
\text { Immersion Test, } 302 / 302 \text { Couple }\end{array}$ \\
\hline & & \multicolumn{8}{|l|}{$\begin{array}{l}\text { Detection } \\
\text { Limit }(g)^{\mathrm{h}}\end{array}$} \\
\hline$d$ & 1 & 0.01 & $0.0113^{*}$ & $29.5^{\mathrm{C}}$ & WL & 302 & 6 & Freshwater & $\begin{array}{l}\text { Gatun Lake, Panama Canal Zone, } \\
\text { Immersion Test, } 302 / 302 \text { Couple }\end{array}$ \\
\hline$d$ & 8 & 0.01 & $0.0014^{*}$ & $29.5^{\mathrm{c}}$ & WL & 302 & 6 & Freshwater & $\begin{array}{l}\text { Gatun Lake, Panama Canal Zone, } \\
\text { Immersion Test, } 302 / 302 \text { Couple }\end{array}$ \\
\hline$d$ & 16 & 0.01 & $0.0007^{*}$ & $29.5^{\mathrm{c}}$ & WL & 302 & 6 & Freshwater & $\begin{array}{l}\text { Gatun Lake, Panama Canal Zone, } \\
\text { Immersion Test, } 302 / 302 \text { Couple }\end{array}$ \\
\hline \multicolumn{10}{|c|}{ mpy } \\
\hline $\mathrm{e}$ & 0.40 & 0.001 & $0.0254^{*}$ & 50 & $\mathrm{WL}$ & $304 \mathrm{~L}$ & 4 & J-13 Well Water & \\
\hline e & 0.57 & 0.009 & $0.2286^{*}$ & 50 & WL & $304 \mathrm{~L}$ & 4 & J-13 Well Water & \\
\hline e & 0.40 & 0.008 & $0.2032^{*}$ & 70 & $\mathrm{WL}$ & $304 \mathrm{~L}$ & 4 & J-13 Well Water & \\
\hline $\mathrm{e}$ & 0.57 & 0.008 & $0.2032^{*}$ & 70 & WL & $304 \mathrm{~L}$ & 4 & J-13 Well Water & \\
\hline $\mathrm{e}$ & 0.40 & 0.008 & $0.2032^{*}$ & 80 & WL & $304 \mathrm{~L}$ & 4 & J-13 Well Water & \\
\hline e & 0.57 & 0.009 & $0.2286^{*}$ & 80 & WL & $304 \mathrm{~L}$ & 4 & J-13 Well Water & \\
\hline e & 0.40 & 0.006 & $0.1524^{*}$ & 90 & WL & $304 \mathrm{~L}$ & 4 & J-13 Well Water & \\
\hline $\mathrm{e}$ & 0.57 & 0.006 & $0.1524^{*}$ & 90 & $\mathrm{WL}$ & $304 \mathrm{~L}$ & 4 & J-13 Well Water & \\
\hline $\mathrm{e}$ & 0.40 & 0.004 & $0.1016^{*}$ & 100 & WL & $304 \mathrm{~L}$ & 4 & J-13 Well Water & \\
\hline e & 0.57 & 0.005 & $0.127^{*}$ & 100 & WL & $304 \mathrm{~L}$ & 4 & $\mathrm{~J}-13$ Well Water & \\
\hline $\mathrm{f}$ & 0.003 & $\mathrm{~N} / \mathrm{A}$ & 0.37 & 90 & EM & $304 \mathrm{~L}$ & 4.8 & Simulated J-13 Well Water & Galvanic Couple of $304 \mathrm{~L}$ and 825 \\
\hline$f$ & 0.003 & $\mathrm{~N} / \mathrm{A}$ & 1.06 & 90 & EM & $304 \mathrm{~L}$ & 4.9 & Solution 10 , Table 2.5 & Galvanic Couple of $304 \mathrm{~L}$ and 825 \\
\hline $\mathrm{f}$ & 0.02 & $\mathrm{~N} / \mathrm{A}$ & 0.49 & 90 & EM & $304 \mathrm{~L}$ & 4.9 & Solution 10 , Table 2.5 & Galvanic Couple of $304 \mathrm{~L}$ and 825 \\
\hline $\mathrm{f}$ & 0.02 & $\mathrm{~N} / \mathrm{A}$ & 0.12 & 90 & EM & $304 \mathrm{~L}$ & 4.8 & Simulated J-13 Well Water & Galvanic Couple of $304 \mathrm{~L}$ and 825 \\
\hline
\end{tabular}


Table 4-2. Stainless Steel Types 302/304/304L Corrosion in Freshwater (Continued)

\begin{tabular}{|c|c|c|c|c|c|c|c|c|c|}
\hline $\begin{array}{l}\text { See } \\
\text { Note }\end{array}$ & $\begin{array}{c}\text { Time } \\
\text { (years) }\end{array}$ & $\begin{array}{c}\text { Corrosion } \\
\text { Rate }\end{array}$ & $\begin{array}{c}\text { Corrosion } \\
\text { Rate }(\mu \mathrm{m} / \mathrm{yr})\end{array}$ & $\begin{array}{c}\text { Temp } \\
\left({ }^{\circ} \mathrm{C}\right)\end{array}$ & $\begin{array}{r}\text { Type of } \\
\text { Analysis }\end{array}$ & $\begin{array}{l}\text { Steel } \\
\text { Type }\end{array}$ & $\begin{array}{l}\text { Table in } \\
\text { Source }\end{array}$ & Fluid Type & Other Comments \\
\hline $\mathrm{f}$ & 0.04 & N/A & 0.46 & 90 & EM & $304 \mathrm{~L}$ & 4.9 & Solution 10 , Table 2.5 & Galvanic Couple of $304 \mathrm{~L}$ and 825 \\
\hline $\mathrm{f}$ & 0.04 & N/A & 0.09 & 90 & EM & $304 \mathrm{~L}$ & 4.8 & Simulated J-13 well water & Galvanic Couple of $304 \mathrm{~L}$ and 825 \\
\hline $\mathrm{f}$ & 0.06 & N/A & 0.47 & 90 & EM & $304 \mathrm{~L}$ & 4.9 & Solution 10 , Table 2.5 & Galvanic Couple of $304 \mathrm{~L}$ and 825 \\
\hline$f$ & 0.06 & $\mathrm{~N} / \mathrm{A}$ & 0.09 & 90 & EM & $304 \mathrm{~L}$ & 4.8 & Simulated J-13 Well Water & Galvanic Couple of $304 \mathrm{~L}$ and 825 \\
\hline $\mathrm{f}$ & 0.07 & $\mathrm{~N} / \mathrm{A}$ & 0.68 & 90 & EM & $304 \mathrm{~L}$ & 4.9 & Solution 10 , Table 2.5 & Galvanic Couple of $304 \mathrm{~L}$ and 825 \\
\hline$f$ & 0.07 & $\mathrm{~N} / \mathrm{A}$ & 0.08 & 90 & EM & $304 \mathrm{~L}$ & 4.8 & Simulated J-13 Well Water & Galvanic Couple of $304 \mathrm{~L}$ and 825 \\
\hline$f$ & 0.09 & N/A & 1.12 & 90 & EM & $304 \mathrm{~L}$ & 4.9 & Solution 10 , Table 2.5 & Galvanic Couple of $304 \mathrm{~L}$ and 825 \\
\hline$f$ & 0.09 & $\mathrm{~N} / \mathrm{A}$ & 0.15 & 90 & EM & $304 \mathrm{~L}$ & 4.8 & Simulated J-13 Well Water & Galvanic Couple of $304 \mathrm{~L}$ and 825 \\
\hline $\mathrm{f}$ & 0.11 & $\mathrm{~N} / \mathrm{A}$ & 0.26 & 90 & EM & $304 \mathrm{~L}$ & 4.9 & Solution 10 , Table 2.5 & Galvanic Couple of $304 \mathrm{~L}$ and 825 \\
\hline$f$ & 0.11 & N/A & $\begin{array}{l}\text { No data } \\
\text { presented }\end{array}$ & 90 & EM & $304 \mathrm{~L}$ & 4.8 & Simulated J-13 Well Water & Galvanic Couple of $304 \mathrm{~L}$ and 825 \\
\hline $\mathrm{f}$ & 0.12 & $\mathrm{~N} / \mathrm{A}$ & 0.07 & 90 & EM & $304 \mathrm{~L}$ & 4.8 & Simulated J-13 Well Water & Galvanic Couple of $304 \mathrm{~L}$ and 825 \\
\hline $\mathrm{f}$ & 0.12 & $\mathrm{~N} / \mathrm{A}$ & 1.29 & 90 & EM & $304 \mathrm{~L}$ & 4.9 & Solution 10 , Table 2.5 & Galvanic Couple of $304 \mathrm{~L}$ and 825 \\
\hline$f$ & 0.13 & $\mathrm{~N} / \mathrm{A}$ & 0.07 & 90 & EM & $304 \mathrm{~L}$ & 4.8 & Simulated J-13 Well Water & Galvanic Couple of $304 \mathrm{~L}$ and 825 \\
\hline$f$ & 0.13 & $\mathrm{~N} / \mathrm{A}$ & 0.21 & 90 & EM & $304 \mathrm{~L}$ & 4.9 & Solution 10 , Table 2.5 & Galvanic Couple of $304 \mathrm{~L}$ and 825 \\
\hline$f$ & 0.16 & $\mathrm{~N} / \mathrm{A}$ & 0.06 & 90 & EM & $304 \mathrm{~L}$ & 4.8 & Simulated J-13 Well Water & Galvanic Couple of $304 \mathrm{~L}$ and 825 \\
\hline $\mathrm{f}$ & 0.16 & $\mathrm{~N} / \mathrm{A}$ & 0.15 & 90 & EM & $304 \mathrm{~L}$ & 4.9 & Solution 10 , Table 2.5 & Galvanic Couple of $304 \mathrm{~L}$ and 825 \\
\hline$f$ & 0.17 & $\mathrm{~N} / \mathrm{A}$ & 0.05 & 90 & EM & $304 \mathrm{~L}$ & 4.8 & Simulated J-13 Well Water & Galvanic Couple of $304 \mathrm{~L}$ and 825 \\
\hline$f$ & 0.17 & $\mathrm{~N} / \mathrm{A}$ & 0.25 & 90 & EM & $304 \mathrm{~L}$ & 4.9 & Solution 10 , Table 2.5 & Galvanic Couple of $304 \mathrm{~L}$ and 825 \\
\hline$f$ & 0.19 & N/A & 0.04 & 90 & EM & $304 \mathrm{~L}$ & 4.8 & Simulated J-13 Well Water & Galvanic Couple of $304 \mathrm{~L}$ and 825 \\
\hline$f$ & 0.19 & $\mathrm{~N} / \mathrm{A}$ & 0.04 & 90 & EM & $304 \mathrm{~L}$ & 4.9 & Solution 10 , Table 2.5 & Galvanic Couple of $304 \mathrm{~L}$ and 825 \\
\hline$f$ & 0.21 & $\mathrm{~N} / \mathrm{A}$ & 0.05 & 90 & EM & $304 \mathrm{~L}$ & 4.8 & Simulated J-13 Well Water & Galvanic Couple of $304 \mathrm{~L}$ and 825 \\
\hline$f$ & 0.21 & $\mathrm{~N} / \mathrm{A}$ & 0.04 & 90 & EM & $304 \mathrm{~L}$ & 4.9 & Solution 10 , Table 2.5 & Galvanic Couple of $304 \mathrm{~L}$ and 825 \\
\hline$f$ & 0.23 & $\mathrm{~N} / \mathrm{A}$ & 0.05 & 90 & EM & $304 \mathrm{~L}$ & 4.8 & Simulated J-13 Well Water & Galvanic Couple of $304 \mathrm{~L}$ and 825 \\
\hline $\mathrm{f}$ & 0.23 & $\mathrm{~N} / \mathrm{A}$ & 0.11 & 90 & EM & $304 \mathrm{~L}$ & 4.9 & Solution 10 , Table 2.5 & Galvanic Couple of $304 \mathrm{~L}$ and 825 \\
\hline $\mathrm{f}$ & 0.23 & $\mathrm{~N} / \mathrm{A}$ & 0.57 & 90 & WL & $304 \mathrm{~L}$ & 4.10 & Simulated J-13 Well Water & Galvanic Couple of $304 \mathrm{~L}$ and 825 \\
\hline$f$ & 0.23 & $\mathrm{~N} / \mathrm{A}$ & 1.57 & 90 & WL & $304 \mathrm{~L}$ & 4.10 & Solution 10 , Table 2.5 & Galvanic Couple of $304 \mathrm{~L}$ and 825 \\
\hline $\mathrm{g}$ & 0.02 & N/A & 0.14 & 90 & EM & $304 \mathrm{~L}$ & 4.2 & $\begin{array}{l}\text { Aerated Simulated J-13 Well } \\
\text { Water }\end{array}$ & \\
\hline $\mathrm{g}$ & 0.04 & $\mathrm{~N} / \mathrm{A}$ & 0.02 & 90 & EM & $304 \mathrm{~L}$ & 4.2 & $\begin{array}{l}\text { Aerated Simulated J-13 Well } \\
\text { Water }\end{array}$ & \\
\hline
\end{tabular}


Table 4-2. Stainless Steel Types 302/304/304L Corrosion in Freshwater (Continued)

\begin{tabular}{|c|c|c|c|c|c|c|c|c|c|}
\hline $\begin{array}{l}\text { See } \\
\text { Note }\end{array}$ & $\begin{array}{c}\begin{array}{c}\text { Time } \\
\text { (years) }\end{array} \\
\end{array}$ & $\begin{array}{c}\text { Corrosion } \\
\text { Rate }\end{array}$ & $\begin{array}{c}\text { Corrosion } \\
\text { Rate }(\mu \mathrm{m} / \mathrm{yr})\end{array}$ & $\begin{array}{c}\text { Temp } \\
\left({ }^{\circ} \mathrm{C}\right)\end{array}$ & $\begin{array}{c}\text { Type of } \\
\text { Analysis }\end{array}$ & $\begin{array}{l}\text { Steel } \\
\text { Type }\end{array}$ & $\begin{array}{l}\text { Table in } \\
\text { Source }\end{array}$ & Fluid Type & Other Comments \\
\hline $\mathrm{g}$ & 0.06 & $\mathrm{~N} / \mathrm{A}$ & 0.02 & 90 & EM & $304 \mathrm{~L}$ & 4.2 & $\begin{array}{l}\text { Aerated Simulated J-13 Well } \\
\text { Water }\end{array}$ & \\
\hline$g$ & 0.17 & N/A & 0.13 & 90 & EM & $304 \mathrm{~L}$ & 4.2 & $\begin{array}{l}\text { Aerated Simulated J-13 Well } \\
\text { Water }\end{array}$ & \\
\hline g & 0.12 & $\mathrm{~N} / \mathrm{A}$ & 0.04 & 90 & EM & $304 \mathrm{~L}$ & 4.2 & $\begin{array}{l}\text { Aerated Simulated J-13 Well } \\
\text { Water }\end{array}$ & \\
\hline g & 0.23 & $\mathrm{~N} / \mathrm{A}$ & 0.06 & 90 & EM & $304 \mathrm{~L}$ & 4.2 & $\begin{array}{l}\text { Aerated Simulated J-13 Well } \\
\text { Water }\end{array}$ & \\
\hline g & 0.11 & $\mathrm{~N} / \mathrm{A}$ & 0.13 & 100 & WL & $304 \mathrm{~L}$ & 7.1 & $\begin{array}{l}\text { Tuff Conditioned Water and } \\
\text { Steam }\end{array}$ & $\begin{array}{l}\text { Reported as "Nil," Which is Less } \\
\text { Than the Weight-Loss Detection } \\
\text { Limit of } 0.13 \text {. Given Value for } \\
\text { Detection Limit }\end{array}$ \\
\hline g & 0.11 & $\mathrm{~N} / \mathrm{A}$ & 0.25 & 100 & WL & $304 \mathrm{~L}$ & 7.1 & $\begin{array}{l}\text { Tuff Conditioned Water and } \\
\text { Steam }\end{array}$ & \\
\hline g & 0.11 & $\mathrm{~N} / \mathrm{A}$ & 0.13 & 100 & $\mathrm{WL}$ & $304 \mathrm{~L}$ & 7.1 & $\begin{array}{l}\text { Tuff Conditioned Water and } \\
\text { Steam }\end{array}$ & $\begin{array}{l}\text { Reported as "Nil," Which is Less } \\
\text { Than the Weight-Loss Detection } \\
\text { Limit of } 0.13 \text {. Given Value for } \\
\text { Detection Limit }\end{array}$ \\
\hline
\end{tabular}

Sources: ${ }^{2}$ McCright et al. 1987 [DIRS 159336].

${ }^{\mathrm{b}}$ Alexander et al. 1961 [DIRS 162265].

${ }^{\circ}$ Forgeson et al. 1958 [DIRS 159343] (Average temperature of Gatun Lake, Panama is approximately $85^{\circ} \mathrm{F}$. This reference is Part 1 of a 5-part series and indicates the temperatures for corrosion studies presented by Alexander et al. 1961 [DIRS 162265] (Table 2) and Southwell et al. 1976 [DIRS 100927]).

dSouthwell et al. 1976 [DIRS 100927].

'Glass et al. 1984 [DIRS 159340].

Beavers et al. 1992 [DIRS 159339].

${ }^{9}$ Beavers and Durr 1991 [DIRS 159341].

hWen the reported corrosion rate is zero, it was set to the detection limit (Southwell et al. 1976 [DIRS 100927]).

NOTE: Values marked with an asterisk (*) calculated in aqueous-304L.XIs in Appendix II.

$\mathrm{WL}=$ weight loss, $\mathrm{EM}=$ electrochemical measurement, $1 \mathrm{mil}=25.4 \mu \mathrm{m}$. 
Table 4-3. Stainless Steel Types 316/316L Corrosion in Freshwater

\begin{tabular}{|c|c|c|c|c|c|c|c|c|c|}
\hline $\begin{array}{l}\text { See } \\
\text { NOTES }\end{array}$ & $\begin{array}{l}\text { Time } \\
\text { (years) }\end{array}$ & $\begin{array}{l}\text { Corrosion } \\
\text { Rate }\end{array}$ & $\begin{array}{c}\text { Corrosion } \\
\text { Rate } \\
(\mu \mathrm{m} / \mathrm{yr}) \\
\end{array}$ & $\begin{array}{l}\text { Temp } \\
\left({ }^{\circ} \mathrm{C}\right)\end{array}$ & $\begin{array}{l}\text { Type of } \\
\text { Analysis }\end{array}$ & $\begin{array}{l}\text { Steel } \\
\text { Type }\end{array}$ & $\begin{array}{l}\text { Table in } \\
\text { Source }\end{array}$ & Fluid Type & Other Comments \\
\hline & & $\begin{array}{l}\text { Detection } \\
{\text { Limit }(g)^{g}}^{\text {gin }}\end{array}$ & & & & & & & \\
\hline a & 1 & 0.01 & $0.0113^{*}$ & $29.5^{\mathrm{c}}$ & WL & 316 & 2 & Gatun Lake (freshwater) & $\begin{array}{l}\text { Panama Canal Zone, Immersion } \\
\text { Test }\end{array}$ \\
\hline a & 2 & 0.01 & $0.0056^{*}$ & $29.5^{\mathrm{c}}$ & WL & 316 & 2 & Gatun Lake (freshwater) & $\begin{array}{l}\text { Panama Canal Zone, Immersion } \\
\text { Test }\end{array}$ \\
\hline a & 4 & 0.01 & $0.0028^{*}$ & $29.5^{\mathrm{c}}$ & WL & 316 & 2 & Gatun Lake (freshwater) & $\begin{array}{l}\text { Panama Canal Zone, Immersion } \\
\text { Test }\end{array}$ \\
\hline a & 8 & 0.01 & $0.0014^{*}$ & $29.5^{\mathrm{c}}$ & WL & 316 & 2 & Gatun Lake (freshwater) & $\begin{array}{l}\text { Panama Canal Zone, Immersion } \\
\text { Test }\end{array}$ \\
\hline $\mathrm{b}$ & 16 & 0.01 & $0.0007^{*}$ & $29.5^{\mathrm{c}}$ & WL & 316 & 4 & Gatun Lake (freshwater) & $\begin{array}{l}\text { Panama Canal Zone, Immersion } \\
\text { Test }\end{array}$ \\
\hline$b$ & 1 & 0.01 & $0.0113^{*}$ & $29.5^{\mathrm{c}}$ & WL & 316 & 6 & Gatun Lake (freshwater) & $\begin{array}{l}\text { Panama Canal Zone, Immersion } \\
\text { Test, Stainless Steel Type } \\
316 / 316 \text { Couple }\end{array}$ \\
\hline$b$ & 8 & 0.01 & $0.0014^{*}$ & $29.5^{\mathrm{c}}$ & WL & 316 & 6 & Gatun Lake (freshwater) & $\begin{array}{l}\text { Panama Canal Zone, Immersion } \\
\text { Test, Stainless Steel Type } \\
\text { 316/316 Couple }\end{array}$ \\
\hline $\mathrm{b}$ & 16 & 0.01 & $0.0007^{*}$ & $29.5^{\mathrm{c}}$ & WL & 316 & 6 & Gatun Lake (freshwater) & $\begin{array}{l}\text { Panama Canal Zone, Immersion } \\
\text { Test, Stainless Steel Type } \\
\text { 316/316 Couple } \\
\end{array}$ \\
\hline $\mathrm{b}$ & 1 & 0.01 & $0.0475^{*}$ & $29.5^{\mathrm{c}}$ & WL & 316 & 6 & Gatun Lake (freshwater) & $\begin{array}{l}\text { Panama Canal Zone, Immersion } \\
\text { Test, Stainless Steel Type } \\
\text { 316/316 Couple } \\
\end{array}$ \\
\hline $\mathrm{b}$ & 8 & 0.01 & $0.0059^{*}$ & $29.5^{\mathrm{c}}$ & WL & 316 & 6 & Gatun Lake (freshwater) & $\begin{array}{l}\text { Panama Canal Zone, Immersion } \\
\text { Test, Stainless Steel Type } \\
\text { 316/316 Couple }\end{array}$ \\
\hline $\mathrm{b}$ & 16 & 0.01 & $0.0030^{*}$ & $29.5^{c}$ & WL & 316 & 6 & Gatun Lake (freshwater) & $\begin{array}{l}\text { Panama Canal Zone, Immersion } \\
\text { Test, Stainless Steel Type } \\
\text { 316/316 Couple }\end{array}$ \\
\hline & & mpy & & & & & & & \\
\hline d & 0.40 & 0.009 & $0.229^{*}$ & 50 & WL & $316 \mathrm{~L}$ & 4 & J-13 Well Water & \\
\hline d & 0.57 & 0.004 & $0.102^{*}$ & 50 & WL & $316 \mathrm{~L}$ & 4 & $\mathrm{~J}-13$ Well Water & \\
\hline d & 0.40 & 0.01 & $0.254^{*}$ & 70 & WL & $316 \mathrm{~L}$ & 4 & J-13 Well Water & \\
\hline
\end{tabular}


Table 4-3. Stainless Steel Types 316/316L Corrosion in Freshwater (Continued)

\begin{tabular}{|c|c|c|c|c|c|c|c|c|c|}
\hline $\begin{array}{l}\text { See } \\
\text { NOTES }\end{array}$ & $\begin{array}{c}\text { Time } \\
\text { (years) }\end{array}$ & $\begin{array}{l}\text { Corrosion } \\
\text { Rate }\end{array}$ & $\begin{array}{c}\text { Corrosion } \\
\text { Rate } \\
(\mu \mathrm{m} / \mathrm{yr})\end{array}$ & $\begin{array}{l}\text { Temp } \\
\left({ }^{\circ} \mathrm{C}\right)\end{array}$ & $\begin{array}{l}\text { Type of } \\
\text { Analysis }\end{array}$ & $\begin{array}{l}\text { Steel } \\
\text { Type }\end{array}$ & $\begin{array}{l}\text { Table in } \\
\text { Source }\end{array}$ & Fluid Type & Other Comments \\
\hline d & 0.57 & 0.009 & $0.229^{*}$ & 70 & WL & $316 \mathrm{~L}$ & 4 & J-13 Well Water & \\
\hline d & 0.40 & 0.011 & $0.279^{*}$ & 80 & WL & $316 \mathrm{~L}$ & 4 & J-13 Well Water & \\
\hline$d$ & 0.57 & 0.01 & $0.254^{*}$ & 80 & WL & $316 \mathrm{~L}$ & 4 & J-13 Well Water & \\
\hline$d$ & 0.40 & 0.006 & $0.152^{*}$ & 90 & WL & $316 \mathrm{~L}$ & 4 & J-13 Well Water & \\
\hline d & 0.57 & 0.01 & $0.254^{*}$ & 90 & WL & $316 \mathrm{~L}$ & 4 & J-13 Well Water & \\
\hline d & 0.40 & 0.007 & $0.178^{*}$ & 100 & WL & $316 \mathrm{~L}$ & 4 & J-13 Well Water & \\
\hline d & 0.57 & 0.008 & $0.203^{*}$ & 100 & WL & $316 \mathrm{~L}$ & 4 & J-13 Well Water & \\
\hline e & 1.31 & $\mathrm{~N} / \mathrm{A}$ & 0.154 & 50 & WL & $316 \mathrm{~L}$ & 6 & J-13 Well Water & Average \\
\hline $\mathrm{e}$ & 1.26 & $\mathrm{~N} / \mathrm{A}$ & 0.109 & 80 & WL & $316 \mathrm{~L}$ & 6 & J-13 Well Water & Average \\
\hline e & 1.18 & $\mathrm{~N} / \mathrm{A}$ & 0.037 & 100 & WL & $316 \mathrm{~L}$ & 6 & J-13 Well Water & Average \\
\hline$f$ & 0.11 & $\mathrm{~N} / \mathrm{A}$ & 0.51 & 100 & EM & $316 \mathrm{~L}$ & 7.1 & $\begin{array}{l}\text { Tuff Conditioned Water and } \\
\text { Steam }\end{array}$ & \\
\hline$f$ & 0.11 & $\mathrm{~N} / \mathrm{A}$ & 0.51 & 100 & EM & $316 \mathrm{~L}$ & 7.1 & $\begin{array}{l}\text { Tuff Conditioned Water and } \\
\text { Steam }\end{array}$ & \\
\hline$f$ & 0.11 & $\mathrm{~N} / \mathrm{A}$ & 0.51 & 100 & EM & $316 \mathrm{~L}$ & 7.1 & $\begin{array}{l}\text { Tuff Conditioned Water and } \\
\text { Steam }\end{array}$ & \\
\hline
\end{tabular}

Sources: ${ }^{a}$ Alexander et al. 1961 [DIRS 162265], Table 2.

bSouthwell et al. 1976 [DIRS 100927].

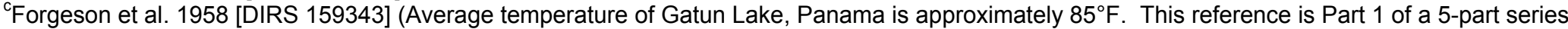
and indicates the temperatures for corrosion studies presented by Alexander et al. 1961 [DIRS 162265] and Southwell et al. 1976 [DIRS 100927 ]).

${ }^{\mathrm{d} G}$ Gass et al. 1984 [DIRS 159340].

${ }^{\mathrm{e}}$ McCright et al. 1987 [DIRS 159336].

'Beavers and Durr 1991 [DIRS 159341].

${ }^{9}$ When the detection limit (Southwell et al. 1976 [DIRS 100927]) is listed for the weight loss, the original corrosion rate was listed as zero.

NOTE: Values marked with an asterisk $\left({ }^{*}\right)$ calculated in aqueous-316L.x/s in Appendix II. $\mathrm{WL}=$ weight loss, $\mathrm{EM}=$ electrochemical measurement. 
Table 4-4. Stainless Steel Types 302/304/304L Corrosion in Seawater

\begin{tabular}{|c|c|c|c|c|c|c|c|c|c|}
\hline $\begin{array}{c}\text { See } \\
\text { NOTES }\end{array}$ & $\begin{array}{c}\text { Time } \\
\text { (years) }\end{array}$ & Corrosion Rate & $\begin{array}{c}\text { Corrosion Rate } \\
(\mu \mathrm{m} / \mathrm{yr})\end{array}$ & $\begin{array}{l}\text { Temp } \\
\text { (C) }\end{array}$ & $\begin{array}{c}\text { Type of } \\
\text { Analysis }\end{array}$ & $\begin{array}{l}\text { Steel } \\
\text { Type }\end{array}$ & $\begin{array}{l}\text { Table in } \\
\text { Source }\end{array}$ & Fluid Type & Other Comments \\
\hline a & 0.022 & N/A & 1.11 & 90 & EM & 304L & 4.7 & $\begin{array}{l}\mathrm{J}-13 \text { Well } \\
\text { Water with } \\
1,000 \mathrm{ppm} \\
\text { Chloride }\end{array}$ & $\begin{array}{l}\text { Galvanic Couple of Stainless } \\
\text { Steel Type } 304 \mathrm{~L} \text { and Carbon } \\
\text { Steel Type C1010 }\end{array}$ \\
\hline a & 0.041 & $\mathrm{~N} / \mathrm{A}$ & 0.66 & 90 & EM & $304 \mathrm{~L}$ & 4.7 & $\begin{array}{l}\mathrm{J}-13 \text { Well } \\
\text { Water with } \\
1,000 \mathrm{ppm} \\
\text { Chloride }\end{array}$ & $\begin{array}{l}\text { Galvanic Couple of Stainless } \\
\text { Steel Type } 304 \mathrm{~L} \text { and Carbon } \\
\text { Steel Type C } 1010\end{array}$ \\
\hline a & 0.060 & $\mathrm{~N} / \mathrm{A}$ & 1.36 & 90 & EM & $304 \mathrm{~L}$ & 4.7 & $\begin{array}{l}\mathrm{J}-13 \text { Well } \\
\text { Water with } \\
1,000 \mathrm{ppm} \\
\text { Chloride }\end{array}$ & $\begin{array}{l}\text { Galvanic Couple of Stainless } \\
\text { Steel Type } 304 \mathrm{~L} \text { and Carbon } \\
\text { Steel Type C1010 }\end{array}$ \\
\hline a & 0.080 & $\mathrm{~N} / \mathrm{A}$ & 1.15 & 90 & EM & $304 \mathrm{~L}$ & 4.7 & $\begin{array}{l}\mathrm{J}-13 \text { Well } \\
\text { Water with } \\
1,000 \mathrm{ppm} \\
\text { Chloride }\end{array}$ & $\begin{array}{l}\text { Galvanic Couple of Stainless } \\
\text { Steel Type } 304 \mathrm{~L} \text { and Carbon } \\
\text { Steel Type C } 1010\end{array}$ \\
\hline a & 0.099 & $\mathrm{~N} / \mathrm{A}$ & 1.65 & 90 & EM & $304 \mathrm{~L}$ & 4.7 & $\begin{array}{l}\mathrm{J}-13 \text { Well } \\
\text { Water with } \\
1,000 \mathrm{ppm} \\
\text { Chloride }\end{array}$ & $\begin{array}{l}\text { Galvanic Couple of Stainless } \\
\text { Steel Type } 304 \mathrm{~L} \text { and Carbon } \\
\text { Steel Type C1010 }\end{array}$ \\
\hline a & 0.117 & N/A & 2.03 & 90 & EM & $304 \mathrm{~L}$ & 4.7 & $\begin{array}{l}\mathrm{J}-13 \text { Well } \\
\text { Water with } \\
1,000 \mathrm{ppm} \\
\text { Chloride }\end{array}$ & $\begin{array}{l}\text { Galvanic Couple of Stainless } \\
\text { Steel Type } 304 \mathrm{~L} \text { and Carbon } \\
\text { Steel Type C } 1010\end{array}$ \\
\hline a & 0.136 & N/A & 4.75 & 90 & EM & $304 \mathrm{~L}$ & 4.7 & $\begin{array}{l}\mathrm{J}-13 \text { Well } \\
\text { Water with } \\
1,000 \mathrm{ppm} \\
\text { Chloride }\end{array}$ & $\begin{array}{l}\text { Galvanic Couple of Stainless } \\
\text { Steel Type } 304 \mathrm{~L} \text { and Carbon } \\
\text { Steel Type C1010 }\end{array}$ \\
\hline a & 0.155 & N/A & 8.2 & 90 & EM & $304 \mathrm{~L}$ & 4.7 & $\begin{array}{l}\mathrm{J}-13 \text { Well } \\
\text { Water with } \\
1,000 \mathrm{ppm} \\
\text { Chloride }\end{array}$ & $\begin{array}{l}\text { Galvanic Couple of Stainless } \\
\text { Steel Type } 304 \mathrm{~L} \text { and C1010 } \\
\text { Carbon Steel }\end{array}$ \\
\hline a & 0.196 & N/A & 15.9 & 90 & EM & $304 \mathrm{~L}$ & 4.7 & $\begin{array}{l}\mathrm{J}-13 \text { Well } \\
\text { Water with } \\
1,000 \text { ppm } \\
\text { Chloride }\end{array}$ & $\begin{array}{l}\text { Galvanic Couple of Stainless } \\
\text { Steel Type } 304 \mathrm{~L} \text { and Carbon } \\
\text { Steel Type C1010 }\end{array}$ \\
\hline
\end{tabular}


Table 4-4. Stainless Steel Types 302/304/304L Corrosion in Seawater (Continued)

\begin{tabular}{|c|c|c|c|c|c|c|c|c|c|}
\hline $\begin{array}{l}\text { See } \\
\text { NOTES }\end{array}$ & $\begin{array}{c}\text { Time } \\
\text { (years) }\end{array}$ & Corrosion Rate & $\begin{array}{c}\text { Corrosion Rate } \\
(\mu \mathrm{m} / \mathrm{yr})\end{array}$ & $\begin{array}{l}\text { Temp } \\
\text { (C) }\end{array}$ & $\begin{array}{c}\text { Type of } \\
\text { Analysis }\end{array}$ & $\begin{array}{l}\text { Steel } \\
\text { Type }\end{array}$ & $\begin{array}{l}\text { Table in } \\
\text { Source }\end{array}$ & Fluid Type & Other Comments \\
\hline a & 0.214 & N/A & 14.84 & 90 & EM & $304 \mathrm{~L}$ & 4.7 & $\begin{array}{l}\mathrm{J}-13 \text { Well } \\
\text { Water with } \\
1,000 \mathrm{ppm} \\
\text { Chloride }\end{array}$ & $\begin{array}{l}\text { Galvanic Couple of Stainless } \\
\text { Steel Type 304L and Carbon } \\
\text { Steel Type C1010 }\end{array}$ \\
\hline a & 0.232 & $\mathrm{~N} / \mathrm{A}$ & 12.33 & 90 & EM & $304 \mathrm{~L}$ & 4.7 & $\begin{array}{l}\mathrm{J}-13 \text { Well } \\
\text { Water with } \\
1,000 \mathrm{ppm} \\
\text { Chloride }\end{array}$ & $\begin{array}{l}\text { Galvanic Couple of Stainless } \\
\text { Steel Type 304L and C1010 }\end{array}$ \\
\hline & & $\left(g / d^{2}\right)$ & & & & & & & \\
\hline $\mathrm{b}$ & 1 & 2.93 & $36.90^{*}$ & $26.7^{\mathrm{c}}$ & WL & 302 & 2 & Seawater & Seawater Immersion \\
\hline $\mathrm{b}$ & 1 & 0.36 & $4.53^{*}$ & $26.7^{\mathrm{c}}$ & WL & 302 & 2 & Seawater & Mean Tide \\
\hline $\mathrm{b}$ & 2 & 4.43 & $27.90^{*}$ & $26.7^{\mathrm{c}}$ & WL & 302 & 2 & Seawater & Seawater Immersion \\
\hline $\mathrm{b}$ & 2 & 0.55 & $3.46^{\star}$ & $26.7^{\mathrm{c}}$ & WL & 302 & 2 & Seawater & Mean Tide \\
\hline $\mathrm{b}$ & 4 & 7.06 & $22.23^{*}$ & $26.7^{\mathrm{c}}$ & WL & 302 & 2 & Seawater & Seawater Immersion \\
\hline b & 4 & 0.99 & $3.12^{*}$ & $26.7^{\mathrm{c}}$ & WL & 302 & 2 & Seawater & Mean Tide \\
\hline $\mathrm{b}$ & 8 & 10.99 & $17.30^{*}$ & $26.7^{\mathrm{c}}$ & WL & 302 & 2 & Seawater & Seawater Immersion \\
\hline $\mathrm{b}$ & 8 & 1.76 & $2.77^{*}$ & $26.7^{\mathrm{c}}$ & $W L$ & 302 & 2 & Seawater & Mean Tide \\
\hline & & $\mathrm{g} / \mathrm{m}^{2}$ & & & & & & & \\
\hline d & 16 & 1870 & $14.72^{*}$ & $26.7^{\mathrm{c}}$ & WL & 302 & 4 & Seawater & Seawater Immersion \\
\hline$d$ & 16 & 330 & $2.60^{*}$ & $26.7^{\mathrm{c}}$ & $W L$ & 302 & 4 & Seawater & Mean Tide \\
\hline & & mils & & & & & & & \\
\hline d & 1 & 0.1 & $2.54^{*}$ & $26.7^{\mathrm{c}}$ & WL & 302 & 6 & Seawater & $\begin{array}{l}\text { Seawater Immersion, Stainless } \\
\text { Steel Type } 302 / 302 \text { Couple }\end{array}$ \\
\hline$d$ & 8 & 5.7 & $18.10^{*}$ & $26.7^{c}$ & WL & 302 & 6 & Seawater & $\begin{array}{l}\text { Seawater Immersion, Stainless } \\
\text { Steel Type 302/302 Couple }\end{array}$ \\
\hline$d$ & 16 & 8.2 & $13.02^{*}$ & $26.7^{c}$ & WL & 302 & 6 & Seawater & $\begin{array}{l}\text { Seawater Immersion, Stainless } \\
\text { Steel Type 302/302 Couple }\end{array}$ \\
\hline$d$ & 1 & 0.2 & $5.08^{*}$ & $26.7^{c}$ & WL & 302 & 6 & Seawater & $\begin{array}{l}\text { Mean Tide, Stainless Steel Type } \\
\text { 302/302 Couple }\end{array}$ \\
\hline$d$ & 8 & 0.5 & $1.59^{\star}$ & $26.7^{c}$ & WL & 302 & 6 & Seawater & $\begin{array}{l}\text { Mean Tide, Stainless Steel Type } \\
\text { 302/302 Couple }\end{array}$ \\
\hline d & 16 & 1.3 & $2.06^{\star}$ & $26.7^{\mathrm{C}}$ & WL & 302 & 6 & Seawater & $\begin{array}{l}\text { Mean Tide, Stainless Steel Type } \\
\text { 302/302 Couple }\end{array}$ \\
\hline
\end{tabular}


Table 4-4. Stainless Steel Types 302/304/304L Corrosion in Seawater (Continued)

\begin{tabular}{|c|c|c|c|c|c|c|c|c|c|}
\hline $\begin{array}{l}\text { See } \\
\text { NOTES }\end{array}$ & $\begin{array}{c}\text { Time } \\
\text { (years) }\end{array}$ & Corrosion Rate & $\begin{array}{l}\text { Corrosion Rate } \\
(\mu \mathrm{m} / \mathrm{yr})\end{array}$ & $\begin{array}{l}\text { Temp } \\
\text { (C) }\end{array}$ & $\begin{array}{l}\text { Type of } \\
\text { Analysis }\end{array}$ & $\begin{array}{l}\text { Steel } \\
\text { Type }\end{array}$ & $\begin{array}{l}\text { Table in } \\
\text { Source }\end{array}$ & Fluid Type & Other Comments \\
\hline$d$ & 1 & 0.3 & $7.62^{*}$ & $26.7^{c}$ & $W L$ & 302 & 6 & Seawater & $\begin{array}{l}\text { Seawater Immersion, Stainless } \\
\text { Steel Type 302/302 Couple }\end{array}$ \\
\hline$d$ & 8 & 4.5 & $14.29^{*}$ & $26.7^{c}$ & WL & 302 & 6 & Seawater & $\begin{array}{l}\text { Seawater Immersion, Stainless } \\
\text { Steel Type 302/302 Couple }\end{array}$ \\
\hline$d$ & 16 & 7.6 & $12.07^{*}$ & $26.7^{c}$ & WL & 302 & 6 & Seawater & $\begin{array}{l}\text { Seawater Immersion, Stainless } \\
\text { Steel Type 302/302 Couple }\end{array}$ \\
\hline d & 1 & 0.1 & $2.54^{*}$ & $26.7^{c}$ & WL & 302 & 6 & Seawater & $\begin{array}{l}\text { Mean Tide, Stainless Steel Type } \\
\text { 302/302 Couple }\end{array}$ \\
\hline$d$ & 8 & 0.7 & $2.22^{*}$ & $26.7^{c}$ & WL & 302 & 6 & Seawater & $\begin{array}{l}\text { Mean Tide, Stainless Steel Type } \\
\text { 302/302 Couple }\end{array}$ \\
\hline d & 16 & 1.2 & $1.91^{*}$ & $26.7^{\mathrm{c}}$ & WL & 302 & 6 & Seawater & $\begin{array}{l}\text { Mean Tide, Stainless Steel Type } \\
\text { 302/302 Couple }\end{array}$ \\
\hline & & mpy & & & & & & & \\
\hline e & 1.32 & 0.08 & $2.032^{*}$ & & WL & 302 & III & $\begin{array}{l}\text { Flowing } \\
\text { Seawater }\end{array}$ & $\begin{array}{l}\text { Flow }=3 \mathrm{ft} / \mathrm{sec} \text { in Trough, Kure } \\
\text { Beach, NC }\end{array}$ \\
\hline e & 4.5 & 0.088 & $2.235^{*}$ & & WL & 302 & III & $\begin{array}{l}\text { Flowing } \\
\text { Seawater }\end{array}$ & $\begin{array}{l}\text { Flow }=3 \mathrm{ft} / \mathrm{sec} \text { in Trough, Kure } \\
\text { Beach, NC }\end{array}$ \\
\hline e & 1.31 & 0.146 & $3.708^{*}$ & & WL & 302 & IV & $\begin{array}{l}\text { Quiet } \\
\text { Seawater }\end{array}$ & Kure Beach, NC \\
\hline & & (mg/dm $/$ day $)$ & & & & & & & \\
\hline$f$ & 0.337 & 5.91 & $27.19^{*}$ & 2.78 & WL & 304 & III & Seawater & Immersion, 5,640 feet \\
\hline$f$ & 2.056 & 8.51 & $39.15^{*}$ & 2.39 & WL & 304 & III & Seawater & Immersion, 5,640 feet \\
\hline$f$ & 2.913 & 5.31 & $24.43^{*}$ & 2.50 & WL & 304 & III & Seawater & Immersion, 5,300 feet \\
\hline$f$ & 1.057 & 3.15 & $14.49^{*}$ & 17.61 & WL & 304 & III & Seawater & Immersion, Surface \\
\hline
\end{tabular}

Sources: ${ }^{a}$ Beavers et al. 1992 [DIRS 159339].

b Alexander et al. 1961 [DIRS 162265].

${ }^{\circ}$ Forgeson et al. 1958 [DIRS 159343] (Average temperature of Pacific Ocean around canal zone, Panama is approximately $80^{\circ} \mathrm{F}$. This reference is Part 1 of a 5-part series and indicates the temperatures for corrosion studies presented by Alexander et al. 1961 [DIRS 162265] and

Southwell et al. 1976 [DIRS 100927]).

d Southwell et al. 1976 [DIRS 100927].

e Bomberger et al. 1954 [DIRS 163699].

Wheatfall 1967 [DIRS 164934].

NOTE: Values marked with an asterisk $\left(^{*}\right)$ calculated in aqueous-304L.x/s in Appendix II. $\mathrm{WL}=$ weight loss, $\mathrm{EM}=$ electrochemical measurement, $1 \mathrm{mil}=25.4 \mu \mathrm{m}$. 
Table 4-5. Stainless Steel Types 316/316L Corrosion in Seawater

\begin{tabular}{|c|c|c|c|c|c|c|c|c|c|}
\hline $\begin{array}{c}\text { See } \\
\text { NOTES }\end{array}$ & $\begin{array}{c}\text { Time } \\
\text { (years) }\end{array}$ & $\begin{array}{c}\text { Corrosion } \\
\text { Rate }\end{array}$ & $\begin{array}{c}\text { Corrosion } \\
\text { Rate }(\mu \mathrm{m} / \mathrm{yr})\end{array}$ & $\begin{array}{c}\text { Temp } \\
\left({ }^{\circ} \mathrm{C}\right)\end{array}$ & $\begin{array}{c}\text { Type of } \\
\text { Analysis }\end{array}$ & $\begin{array}{l}\text { Steel } \\
\text { Type }\end{array}$ & $\begin{array}{l}\text { Table in } \\
\text { Source }\end{array}$ & Fluid Type & Other Comments \\
\hline & & mpy & & & & & & & \\
\hline$a$ & 1.32 & 0.16 & $4.064^{*}$ & & WL & 316 & III & Seawater & Kure Beach, NC \\
\hline$a$ & 4.5 & 0.061 & $1.549^{*}$ & & WL & 316 & III & Seawater & Kure Beach, NC \\
\hline \multirow[t]{2}{*}{$a$} & 1.31 & 0.24 & $6.096^{*}$ & & WL & 316 & IV & Seawater & Kure Beach, NC \\
\hline & & (g/dm²/yr) & & & & & & & \\
\hline$b$ & 1 & 1.18 & $14.79^{*}$ & $26.7^{\circ}$ & WL & 316 & 2 & Seawater & Canal Zone, Immersion \\
\hline$b$ & 2 & 0.65 & $4.07^{*}$ & $26.7^{\circ}$ & $\mathrm{WL}$ & 316 & 2 & Seawater & Canal Zone, Immersion \\
\hline $\mathrm{b}$ & 4 & 0.54 & $1.69^{*}$ & $26.7^{\circ}$ & WL & 316 & 2 & Seawater & Canal Zone, Immersion \\
\hline $\mathrm{b}$ & 8 & 4.08 & $6.39^{*}$ & $26.7^{\circ}$ & $\mathrm{WL}$ & 316 & 2 & Seawater & Canal Zone, Immersion \\
\hline$b$ & 1 & 0.13 & $1.63^{*}$ & $26.7^{\circ}$ & $\mathrm{WL}$ & 316 & 2 & Seawater & Canal Zone, Mean Tide \\
\hline$b$ & 2 & 0.1 & $0.63^{*}$ & $26.7^{\mathrm{C}}$ & WL & 316 & 2 & Seawater & Canal Zone, Mean Tide \\
\hline$b$ & 4 & 0.27 & $0.85^{*}$ & $26.7^{\mathrm{C}}$ & WL & 316 & 2 & Seawater & Canal Zone, Mean Tide \\
\hline $\mathrm{b}$ & 8 & 0.39 & $0.61^{*}$ & $26.7^{\circ}$ & WL & 316 & 2 & Seawater & Canal Zone, Mean Tide \\
\hline & & $\mathrm{g} / \mathrm{m}^{2}$ & & & & & & & \\
\hline $\mathrm{d}$ & 16 & 160 & $1.25^{*}$ & $26.7^{\circ}$ & WL & 316 & 4 & Seawater & Canal Zone, Immersion \\
\hline$d$ & 16 & 20 & $0.16^{*}$ & $26.7^{\circ}$ & WL & 316 & 4 & Seawater & Canal Zone, Mean Tide \\
\hline & & mils & & & & & & & \\
\hline$d$ & 8 & 0.3 & $0.9525^{*}$ & $26.7^{\mathrm{C}}$ & WL & 316 & 6 & Seawater & $\begin{array}{l}\text { Canal Zone, Immersion, Stainless Steel } \\
\text { Type } 316 / 316 \text { Couple }\end{array}$ \\
\hline$d$ & 16 & 3.1 & $4.92125^{\star}$ & $26.7^{\mathrm{C}}$ & WL & 316 & 6 & Seawater & $\begin{array}{l}\text { Canal Zone, Immersion, Stainless Steel } \\
\text { Type } 316 / 316 \text { Couple }\end{array}$ \\
\hline$d$ & 8 & 0.1 & $0.3175^{*}$ & $26.7^{\mathrm{C}}$ & WL & 316 & 6 & Seawater & $\begin{array}{l}\text { Canal Zone, Mean Tide, Stainless Steel } \\
\text { Type } 316 / 316 \text { Couple }\end{array}$ \\
\hline$d$ & 16 & 0.1 & $0.15875^{\star}$ & $26.7^{\mathrm{C}}$ & WL & 316 & 6 & Seawater & $\begin{array}{l}\text { Canal Zone, Mean Tide, Stainless Steel } \\
\text { Type } 316 / 316 \text { Couple }\end{array}$ \\
\hline$d$ & 8 & 0.6 & $1.905^{*}$ & $26.7^{\mathrm{C}}$ & WL & 316 & 6 & Seawater & $\begin{array}{l}\text { Canal Zone, Immersion, Stainless Steel } \\
\text { Type } 316 / 316 \text { Couple }\end{array}$ \\
\hline$d$ & 16 & 1.3 & $2.06375^{\star}$ & $26.7^{\circ}$ & WL & 316 & 6 & Seawater & $\begin{array}{l}\text { Canal Zone, Immersion, Stainless Steel } \\
\text { Type } 316 / 316 \text { Couple }\end{array}$ \\
\hline$d$ & 16 & 0.1 & $0.15875^{*}$ & $26.7^{\circ}$ & WL & 316 & 6 & Seawater & $\begin{array}{l}\text { Canal Zone, Mean Tide, Stainless Steel } \\
\text { Type } 316 / 316 \text { Couple }\end{array}$ \\
\hline
\end{tabular}




\begin{tabular}{|c|c|c|c|c|c|c|c|c|c|}
\hline $\begin{array}{c}\text { See } \\
\text { NOTES }\end{array}$ & $\begin{array}{c}\text { Time } \\
\text { (years) }\end{array}$ & $\begin{array}{l}\text { Corrosion } \\
\text { Rate }\end{array}$ & $\begin{array}{c}\text { Corrosion } \\
\text { Rate }(\mu \mathrm{m} / \mathrm{yr})\end{array}$ & $\begin{array}{c}\text { Temp } \\
\left({ }^{\circ} \mathrm{C}\right)\end{array}$ & $\begin{array}{l}\text { Type of } \\
\text { Analysis }\end{array}$ & $\begin{array}{l}\text { Steel } \\
\text { Type }\end{array}$ & $\begin{array}{l}\text { Table in } \\
\text { Source }\end{array}$ & Fluid Type & Other Comments \\
\hline & & $\begin{array}{l}\text { g (detection } \\
\text { limit) }^{\mathrm{e}}\end{array}$ & & & & & & & \\
\hline$d$ & 1 & 0.01 & $0.05^{*}$ & $26.7^{c}$ & WL & 316 & 6 & Seawater & $\begin{array}{l}\text { Canal Zone, Immersion, Stainless Steel } \\
\text { Type } 316 / 316 \text { Couple }\end{array}$ \\
\hline$d$ & 1 & 0.01 & $0.05^{\star}$ & $26.7^{c}$ & WL & 316 & 6 & Seawater & $\begin{array}{l}\text { Canal Zone, Mean Tide, Stainless Steel } \\
\text { Type } 316 / 316 \text { Couple }\end{array}$ \\
\hline$d$ & 1 & 0.01 & $0.01^{*}$ & $26.7^{c}$ & WL & 316 & 6 & Seawater & $\begin{array}{l}\text { Canal Zone, Immersion, Stainless Steel } \\
\text { Type } 316 / 316 \text { Couple }\end{array}$ \\
\hline$d$ & 1 & 0.01 & $0.01^{*}$ & $26.7^{c}$ & WL & 316 & 6 & Seawater & $\begin{array}{l}\text { Canal Zone, Mean Tide, Stainless Steel } \\
\text { Type } 316 / 316 \text { Couple }\end{array}$ \\
\hline$d$ & 8 & 0.01 & $0.001^{*}$ & $26.7^{c}$ & WL & 316 & 6 & Seawater & $\begin{array}{l}\text { Canal Zone, Mean Tide, Stainless Steel } \\
\text { Type } 316 / 316 \text { Couple }\end{array}$ \\
\hline$f$ & 0.11 & $\mathrm{~N} / \mathrm{A}$ & 0.51 & 100 & EM & $316 \mathrm{~L}$ & 7.1 & $\begin{array}{l}\text { Tuff Conditioned } \\
\text { Water and Steam }\end{array}$ & \\
\hline$f$ & 0.11 & $\mathrm{~N} / \mathrm{A}$ & 0.51 & 100 & EM & $316 \mathrm{~L}$ & 7.1 & $\begin{array}{l}\text { Tuff Conditioned } \\
\text { Water and Steam }\end{array}$ & \\
\hline
\end{tabular}

Sources: a Bomberger et al. 1954 [DIRS 163699].

${ }^{b}$ Alexander et al. 1961 [DIRS 162265].

${ }^{\mathrm{C}}$ Forgeson et al. 1958 [DIRS 159343] (Average temperature of pacific ocean around canal zone, Panama is approximately $80^{\circ} \mathrm{F}$. This reference is Part 1 of a 5-part series and indicates the temperatures for corrosion studies presented in Alexander et al. 1961 [DIRS 162265] and Southwell et al. 1976 [DIRS 100927]).

d Southwell et al. 1976 [DIRS 100927].

e When the reported corrosion rate is zero, it was set to the detection limit (Southwell et al. 1976 [DIRS 100927]).

f Beavers and Durr 1991 [DIRS 159341].

NOTE: Values marked with an asterisk (*) are calculated in aqueous-304L.x/s in Appendix II.

$\mathrm{WL}=$ weight loss, $\mathrm{EM}=$ electrochemical measurement, $1 \mathrm{mil}=25.4 \mu \mathrm{m}$. 
Table 4-6. Corrosion of Stainless Steel Type 304L in Boil-Down Tests

\begin{tabular}{|c|c|c|c|c|c|l|}
\hline $\begin{array}{c}\text { Time } \\
(\mathbf{y e a r s})\end{array}$ & $\begin{array}{c}\text { Corrosion Rate } \\
(\boldsymbol{\mu \mathrm { m }} / \mathbf{y r})\end{array}$ & ${\text { Temp }\left({ }^{\circ} \mathbf{C}\right)}$ & Type of Analysis & Steel Type & $\begin{array}{c}\text { Table in } \\
\text { Source }\end{array}$ & \multicolumn{1}{|c|}{ Fluid Type } \\
\hline 0.04 & 0.1 & 90 & EM & $304 \mathrm{~L}$ & 5.4 & Aerated J-13 Well Water \\
\hline 0.08 & 0.09 & 90 & EM & $304 \mathrm{~L}$ & 5.4 & Aerated J-13 Well Water \\
\hline 0.17 & 0.26 & 90 & EM & $304 \mathrm{~L}$ & 5.4 & Aerated J-13 Well Water \\
\hline 0.23 & 0.13 & 90 & EM & $304 \mathrm{~L}$ & 5.4 & Aerated J-13 Well Water \\
\hline 0.31 & 1.08 & 90 & EM & $304 \mathrm{~L}$ & 5.4 & Aerated J-13 Well Water \\
\hline 0.38 & 1.28 & 90 & EM & $304 \mathrm{~L}$ & 5.4 & Aerated J-13 Well Water \\
\hline 0.46 & 0.6 & 90 & EM & $304 \mathrm{~L}$ & 5.4 & Aerated J-13 Well Water \\
\hline 0.54 & 11.8 & 90 & EM & $304 \mathrm{~L}$ & 5.4 & Aerated J-13 Well Water \\
\hline 0.61 & 0.78 & 90 & EM & $304 \mathrm{~L}$ & 5.4 & Aerated J-13 Well Water \\
\hline 0.71 & 7.71 & 90 & EM & $304 \mathrm{~L}$ & 5.4 & Aerated J-13 Well Water \\
\hline 0.77 & 4.49 & 90 & EM & $304 \mathrm{~L}$ & 5.4 & Aerated J-13 Well Water \\
\hline 0.84 & 8.76 & 90 & EM & $304 \mathrm{~L}$ & 5.4 & Aerated J-13 Well Water \\
\hline 0.92 & 3.34 & 90 & EM & $304 \mathrm{~L}$ & 5.4 & Aerated J-13 Well Water \\
\hline 1.00 & 2.25 & 90 & EM & $304 \mathrm{~L}$ & 5.4 & Aerated J-13 Well Water \\
\hline 1.07 & 2.13 & 90 & EM & $304 \mathrm{~L}$ & 5.4 & Aerated J-13 Well Water \\
\hline 1.15 & 3.36 & 90 & EM & $304 \mathrm{~L}$ & 5.4 & Aerated J-13 Well Water \\
\hline 1.23 & 3.14 & 90 & EM & $304 \mathrm{~L}$ & 5.4 & Aerated J-13 Well Water \\
\hline 1.32 & 3.54 & 90 & EM & $304 \mathrm{~L}$ & 5.4 & Aerated J-13 Well Water \\
\hline 1.38 & 1.93 & 90 & EM & $304 \mathrm{~L}$ & 5.4 & Aerated J-13 Well Water \\
\hline 1.48 & 2.31 & 90 & EM & $304 \mathrm{~L}$ & 5.4 & Aerated J-13 Well Water \\
\hline 1.53 & 2.32 & 90 & EM & $304 \mathrm{~L}$ & 5.4 & Aerated J-13 Well Water \\
\hline
\end{tabular}

Source: Beavers et al. 1992 [DIRS 159339].

NOTE: $E M=$ electrochemical measurement. 
Table 4-7. Corrosion Rates of Stainless Steel Type 304L in Solution Containing Radiolysis Products $\left(\mathrm{H}_{2} \mathrm{O}_{2}\right)$

\begin{tabular}{|c|c|c|c|c|c|c|c|}
\hline $\begin{array}{c}\text { Time } \\
\text { (years) }\end{array}$ & $\begin{array}{c}\text { Corrosion } \\
\text { Rate }(\mu \mathrm{m} / \mathrm{yr})\end{array}$ & $\begin{array}{c}\text { Temp } \\
\left({ }^{\circ} \mathrm{C}\right)\end{array}$ & $\begin{array}{l}\text { Type of } \\
\text { Analysis }\end{array}$ & $\begin{array}{l}\text { Steel } \\
\text { Type }\end{array}$ & $\begin{array}{c}\text { Table in } \\
\text { Source }\end{array}$ & Fluid Type & $\begin{array}{c}\text { Other } \\
\text { Comments }\end{array}$ \\
\hline 0.29 & 0.04 & 90 & EM & $304 \mathrm{~L}$ & 5.5 & \multirow{7}{*}{$\begin{array}{l}\text { Solution } 20 \text { (Main } \\
\text { Components = } 200 \text { ppm F-, } \\
1,000 \text { ppm } \mathrm{Cl}^{-}, 200 \text { ppm } \mathrm{NO}_{2}^{-} \text {, } \\
\text { and } 408 \mathrm{ppm} \mathrm{K}^{+} \text {) }\end{array}$} & $0 \mathrm{ppm} \mathrm{\textrm {H } _ { 2 } \mathrm { O } _ { 2 }}$ \\
\hline 0.29 & 0.18 & 90 & EM & $304 \mathrm{~L}$ & 5.5 & & $200 \mathrm{ppm} \mathrm{H}_{2} \mathrm{O}_{2}$ \\
\hline 0.30 & 0.6 & 90 & EM & $304 \mathrm{~L}$ & 5.5 & & $400 \mathrm{ppm} \mathrm{\textrm {H } _ { 2 } \mathrm { O } _ { 2 }}$ \\
\hline 0.30 & 0.88 & 90 & EM & $304 \mathrm{~L}$ & 5.5 & & $600 \mathrm{ppm} \mathrm{\textrm {H } _ { 2 } \mathrm { O } _ { 2 }}$ \\
\hline 0.31 & 1.16 & 90 & EM & $304 \mathrm{~L}$ & 5.5 & & $1,200 \mathrm{ppm} \mathrm{H} \mathrm{O}_{2}$ \\
\hline 0.31 & 3.44 & 90 & EM & $304 \mathrm{~L}$ & 5.5 & & $2,400 \mathrm{ppm} \mathrm{H}_{2} \mathrm{O}_{2}$ \\
\hline 0.32 & 6.58 & 90 & EM & $304 \mathrm{~L}$ & 5.5 & & 4,800 ppm $\mathrm{H}_{2} \mathrm{O}_{2}$ \\
\hline
\end{tabular}

Source: Beavers and Durr 1991 [DIRS 159341].

NOTE: $E M=$ electrochemical measurement. 
Table 4-8. Neutronit-Corrosion Rates (Using Stainless Steel Type 321 Surrogate) in Freshwater

\begin{tabular}{|c|c|c|c|c|c|c|c|c|}
\hline $\begin{array}{l}\text { See } \\
\text { Note }\end{array}$ & $\begin{array}{c}\text { Time } \\
\text { (years) }\end{array}$ & $\begin{array}{l}\text { Corrosion } \\
\text { Rate }\end{array}$ & $\begin{array}{c}\text { Corrosion Rate } \\
(\mu \mathrm{m} / \mathrm{yr})\end{array}$ & $\begin{array}{l}\text { Temp } \\
\left({ }^{\circ} \mathrm{C}\right)\end{array}$ & $\begin{array}{l}\text { Type of } \\
\text { Analysis }\end{array}$ & $\begin{array}{l}\text { Table From } \\
\text { Reference }\end{array}$ & $\begin{array}{l}\text { Steel } \\
\text { Type }\end{array}$ & Fluid Type \\
\hline & & $\begin{array}{l}\text { Detection } \\
\text { Limit }(g)^{\mathrm{e}}\end{array}$ & & & & & & \\
\hline a & 2 & 0.01 & $0.006^{*}$ & $29.5^{\mathrm{d}}$ & $W L$ & 2 & 321 & $\begin{array}{l}\text { Gatun Lake, Panama Canal Zone, } \\
\text { Freshwater Lake }\end{array}$ \\
\hline a & 4 & 0.01 & $0.003^{*}$ & $29.5^{d}$ & WL & 2 & 321 & $\begin{array}{l}\text { Gatun Lake, Panama Canal Zone, } \\
\text { Freshwater Lake }\end{array}$ \\
\hline a & 1 & 0.01 & $0.01^{*}$ & $29.5^{d}$ & WL & 2 & 321 & $\begin{array}{l}\text { Gatun Lake, Panama Canal Zone, } \\
\text { Freshwater Lake }\end{array}$ \\
\hline$b$ & 8 & 0.01 & $0.001^{*}$ & $29.5^{d}$ & WL & 2 & 321 & $\begin{array}{l}\text { Gatun Lake, Panama Canal Zone, } \\
\text { Freshwater Lake }\end{array}$ \\
\hline$b$ & 16 & 0.01 & $0.0007^{*}$ & $29.5^{d}$ & WL & 4 & 321 & $\begin{array}{l}\text { Gatun Lake, Panama Canal Zone, } \\
\text { Freshwater Lake }\end{array}$ \\
\hline & & mils/yr & & & & & & \\
\hline $\mathrm{c}$ & 0.405 & 0.007 & $0.2^{*}$ & 50 & WL & 4 & 321 & J-13 Well Water \\
\hline $\mathrm{c}$ & 0.570 & 0.005 & $0.1^{*}$ & 50 & WL & 4 & 321 & J-13 Well Water \\
\hline c & 0.405 & 0.012 & $0.30^{*}$ & 70 & WL & 4 & 321 & J-13 Well Water \\
\hline $\mathrm{c}$ & 0.570 & 0.011 & $0.28^{*}$ & 70 & WL & 4 & 321 & J-13 Well Water \\
\hline $\mathrm{c}$ & 0.405 & 0.008 & $0.2^{*}$ & 80 & WL & 4 & 321 & J-13 Well Water \\
\hline $\mathrm{c}$ & 0.570 & 0.008 & $0.2^{*}$ & 80 & WL & 4 & 321 & J-13 Well Water \\
\hline c & 0.405 & 0.008 & $0.2^{*}$ & 90 & WL & 4 & 321 & J-13 Well Water \\
\hline c & 0.570 & 0.013 & $0.33^{*}$ & 90 & WL & 4 & 321 & J-13 Well Water \\
\hline $\mathrm{c}$ & 0.405 & 0.008 & $0.2^{*}$ & 100 & WL & 4 & 321 & J-13 Well Water \\
\hline c & 0.570 & 0.001 & $0.03^{*}$ & 100 & WL & 4 & 321 & J-13 Well Water \\
\hline
\end{tabular}

Sources: ${ }^{a}$ Alexander et al. 1961 [DIRS 162265].

${ }^{b}$ Southwell et al. 1976 [DIRS 100927]

${ }^{c}$ Glass et al. 1984 [DIRS 159340].

d Forgeson et al. 1958 [DIRS 159343].

e When the reported corrosion rate is zero, it was set to the detection limit (Southwell et al. 1976 [DIRS 100927]). 
Table 4-9. Neutronit Corrosion Rates (Using Stainless Steel Type 321 Surrogate) in Seawater

\begin{tabular}{|c|c|c|c|c|c|c|c|c|}
\hline $\begin{array}{l}\text { See } \\
\text { Note }\end{array}$ & $\begin{array}{c}\text { Time } \\
\text { (years) }\end{array}$ & $\begin{array}{c}\text { Corrosion } \\
\text { Rate }\end{array}$ & $\begin{array}{c}\text { Corrosion Rate } \\
(\mu \mathrm{m} / \mathrm{yr})\end{array}$ & $\begin{array}{c}\text { Temp } \\
\left({ }^{\circ} \mathrm{C}\right)\end{array}$ & $\begin{array}{c}\text { Type of } \\
\text { Analysis }\end{array}$ & $\begin{array}{c}\text { Table From } \\
\text { Reference }\end{array}$ & $\begin{array}{l}\text { Steel } \\
\text { Type }\end{array}$ & Fluid Type \\
\hline & & $\mathrm{g} / \mathrm{m}^{2}$ & & & & & & \\
\hline a & 16 & 1,460 & $11.49^{*}$ & $26.7^{\mathrm{c}}$ & WL & 4 & 321 & $\begin{array}{l}\text { Seawater, Panama Canal } \\
\text { Zone/Immersion }\end{array}$ \\
\hline \multirow[t]{2}{*}{ a } & 16 & 230 & $1.81^{*}$ & $26.7^{c}$ & WL & 4 & 321 & $\begin{array}{l}\text { Seawater, Panama Canal Zone/Mean } \\
\text { Tide }\end{array}$ \\
\hline & & $\mathrm{g} / \mathrm{dm}^{2}$ & & & & & & \\
\hline $\mathrm{b}$ & 1 & 2.32 & $29.2^{*}$ & $26.7^{\mathrm{c}}$ & WL & 2 & 321 & $\begin{array}{l}\text { Seawater, Panama Canal } \\
\text { Zone/Immersion }\end{array}$ \\
\hline$b$ & 2 & 3.44 & $21.7^{\star}$ & $26.7^{c}$ & WL & 2 & 321 & $\begin{array}{l}\text { Seawater, Panama Canal } \\
\text { Zone/Immersion }\end{array}$ \\
\hline$b$ & 4 & 6.55 & $20.6^{*}$ & $26.7^{c}$ & WL & 2 & 321 & $\begin{array}{l}\text { Seawater, Panama Canal } \\
\text { Zone/Immersion }\end{array}$ \\
\hline$b$ & 8 & 10.02 & $15.77^{*}$ & $26.7^{\mathrm{c}}$ & WL & 2 & 321 & $\begin{array}{l}\text { Seawater, Panama Canal } \\
\text { Zone/Immersion }\end{array}$ \\
\hline$b$ & 1 & 0.26 & $3.3^{*}$ & $26.7^{c}$ & WL & 2 & 321 & $\begin{array}{l}\text { Seawater, Panama Canal Zone/Mean } \\
\text { Tide }\end{array}$ \\
\hline$b$ & 2 & 0.4 & $3^{*}$ & $26.7^{c}$ & WL & 2 & 321 & $\begin{array}{l}\text { Seawater, Panama Canal Zone/Mean } \\
\text { Tide }\end{array}$ \\
\hline$b$ & 4 & 0.67 & $2.1^{*}$ & $26.7^{c}$ & WL & 2 & 321 & $\begin{array}{l}\text { Seawater, Panama Canal Zone/Mean } \\
\text { Tide }\end{array}$ \\
\hline b & 8 & 1.34 & $2.11^{*}$ & $26.7^{\mathrm{c}}$ & WL & 2 & 321 & $\begin{array}{l}\text { Seawater, Panama Canal Zone/Mean } \\
\text { Tide }\end{array}$ \\
\hline
\end{tabular}

Sources: a Southwell et al. 1976 [DIRS 100927].

${ }^{\mathrm{b}}$ Alexander et al. 1961 [DIRS 162265].

'Forgeson et al. 1958 [DIRS 159343].

NOTE: Values marked with an asterisk $\left(^{*}\right)$ calculated in aqueous-B-Gd-steels.xls in Appendix II.

$\mathrm{WL}=$ weight loss, $\mathrm{EM}=$ electrochemical measurement. 
Table 4-10. Corrosion of Borated Stainless Steel Type 304L for Use as "Freshwater" Rates

\begin{tabular}{|c|c|c|c|c|c|c|c|}
\hline $\begin{array}{l}\text { Corrosion Rate } \\
\text { (mils/month) }\end{array}$ & $\begin{array}{l}\text { Corrosion Rate } \\
(\mu \mathrm{m} / \mathrm{yr})\end{array}$ & $\begin{array}{l}\text { Temp } \\
\left({ }^{\circ} \mathrm{C}\right)\end{array}$ & $\underset{B}{\text { Wt. } \%}$ & $\begin{array}{l}\text { Steel } \\
\text { Type }\end{array}$ & $\begin{array}{l}\text { Solution } \\
\text { Type }\end{array}$ & $\begin{array}{l}\text { Table in } \\
\text { Source }\end{array}$ & Other Comments \\
\hline 0.67 & $200^{*}$ & Boiling & 1.5 & 304 & 6 & VII & \\
\hline 0.72 & $220^{*}$ & Boiling & 1.5 & 304 & 6 & VII & \\
\hline 0.62 & $190^{*}$ & Boiling & 1.5 & 304 & 6 & VII & With Chemically Sacrificial Zirconium \\
\hline 0.53 & $160^{*}$ & Boiling & 1.5 & 304 & 6 & VII & With Chemically Sacrificial Zirconium \\
\hline 2.36 & $719^{*}$ & Ambient & 1.5 & 304 & 11 & VIII & \\
\hline 2.53 & $771^{*}$ & Ambient & 1.5 & 304 & 11 & VIII & \\
\hline 0.77 & $230^{*}$ & Ambient & 1.5 & 304 & 11 & VIII & With Chemically Sacrificial Zirconium \\
\hline 0.83 & $250^{*}$ & Ambient & 1.5 & 304 & 11 & VIII & With Chemically Sacrificial Zirconium \\
\hline 0.01 & $3^{*}$ & 50 & 0.3 & 304 & 11 & VIII & \\
\hline 0.01 & $3^{*}$ & 50 & 0.3 & 304 & 11 & VIII & \\
\hline 0.04 & $10^{*}$ & 50 & 0.3 & 304 & 11 & VIII & Stainless Steel Type 304 Welded with ER-310 Rod \\
\hline 0.06 & $20^{*}$ & 50 & 0.3 & 304 & 11 & VIII & Stainless Steel Type 304 Welded with ER-310 Rod \\
\hline 0.09 & $30^{*}$ & 50 & 0.3 & 304 & 11 & VIII & $\begin{array}{l}\text { Stainless Steel Type } 304 \text { Welded to Stainless Steel } \\
\text { Type } 304 \text { with ER- } 310 \text { Rod }\end{array}$ \\
\hline 0.07 & $20^{*}$ & 50 & 0.3 & 304 & 11 & VIII & $\begin{array}{l}\text { Stainless Steel Type } 304 \text { Welded to Stainless Steel } \\
\text { Type } 304 \text { with ER-310 Rod }\end{array}$ \\
\hline 0.04 & $10^{*}$ & Boiling & 0.3 & 304 & 6 & VII & \\
\hline 0.03 & $9^{*}$ & Boiling & 0.3 & 304 & 6 & VII & \\
\hline 0.07 & $20^{*}$ & Boiling & 0.3 & 304 & 6 & VII & Stainless Steel Type 304 Welded with ER-310 Rod \\
\hline 0.05 & $20^{*}$ & Boiling & 0.3 & 304 & 6 & VII & Stainless Steel Type 304 Welded with ER-310 Rod \\
\hline 0.08 & $20^{*}$ & Boiling & 0.3 & 304 & 6 & VII & $\begin{array}{l}\text { Stainless Steel Type } 304 \text { Welded to Stainless Steel } \\
\text { Type } 304 \text { With ER-310 Rod }\end{array}$ \\
\hline 0.07 & $20^{*}$ & Boiling & 0.3 & 304 & 6 & VII & $\begin{array}{l}\text { Stainless Steel Type } 304 \text { Welded to Stainless Steel } \\
\text { Type } 304 \text { With ER-310 Rod }\end{array}$ \\
\hline 0.01 & $3^{*}$ & Ambient & 0.3 & 304 & 11 & VIII & \\
\hline 0.01 & $3^{*}$ & Ambient & 0.3 & 304 & 11 & VIII & \\
\hline 0.01 & $3^{*}$ & Ambient & 0.3 & 304 & 11 & VIIII & Stainless Steel Type 304 Welded with ER-310 Rod \\
\hline 0.02 & $6^{*}$ & Ambient & 0.3 & 304 & 11 & VIII & Stainless Steel Type 304 Welded with ER-310 Rod \\
\hline 0.04 & $10^{*}$ & Ambient & 0.3 & 304 & 11 & VIII & $\begin{array}{l}\text { Stainless Steel Type } 304 \text { Welded to Stainless Steel } \\
\text { Type } 304 \text { with ER-310 Rod }\end{array}$ \\
\hline 0.03 & $10^{*}$ & Ambient & 0.3 & 304 & 11 & VIII & $\begin{array}{l}\text { Stainless Steel Type } 304 \text { Welded to Stainless Steel } \\
\text { Type } 304 \text { with ER-310 Rod }\end{array}$ \\
\hline
\end{tabular}

Source: Cole 1976 [DIRS 159369].

NOTES: For more information on the solution type, see aqueous-B-Gd-steels.x/s in Appendix II. Values marked with an asterisk $\left(^{*}\right)$ calculated in aqueous-B-Gd-steels.Xls in Appendix II. 
Table 4-11. Corrosion of Borated Stainless Steel Type 304L for Use as "Saltwater" Rates

\begin{tabular}{|c|c|c|c|c|c|c|c|}
\hline $\begin{array}{c}\text { Corrosion Rate } \\
\text { (mils/month) }\end{array}$ & $\begin{array}{c}\text { Corrosion Rate } \\
(\mu \mathrm{m} / \mathrm{yr})\end{array}$ & $\begin{array}{l}\text { Temp } \\
\left({ }^{\circ} \mathrm{C}\right)\end{array}$ & $\begin{array}{c}\text { Wt. \% } \\
\text { B }\end{array}$ & $\begin{array}{l}\text { Steel } \\
\text { Type }\end{array}$ & $\begin{array}{c}\text { Solution } \\
\text { Type }\end{array}$ & $\begin{array}{l}\text { Table in } \\
\text { Source }\end{array}$ & Other Comments \\
\hline 1.95 & $573^{*}$ & 100 & 1.5 & 304 & 2 & VII & \\
\hline 1.51 & $444^{*}$ & 100 & 1.5 & 304 & 2 & VII & \\
\hline 2.54 & $747^{*}$ & 100 & 1.5 & 304 & 3 & VII & \\
\hline 1.91 & $562^{*}$ & 100 & 1.5 & 304 & 3 & VII & \\
\hline 6.04 & $1,780^{*}$ & 100 & 1.5 & 304 & 5 & VII & \\
\hline 6.44 & $1,890^{*}$ & 100 & 1.5 & 304 & 5 & VII & \\
\hline 0.92 & $270^{*}$ & 100 & 1.5 & 304 & 9 & VII & \\
\hline 1.03 & $303^{*}$ & 100 & 1.5 & 304 & 9 & VII & \\
\hline 0.58 & $170^{*}$ & 100 & 1.5 & 304 & 2 & VII & $\begin{array}{l}\text { With Chemically } \\
\text { Sacrificial Zirconium }\end{array}$ \\
\hline 0.72 & $210^{*}$ & 100 & 1.5 & 304 & 2 & VII & $\begin{array}{l}\text { With Chemically } \\
\text { Sacrificial Zirconium }\end{array}$ \\
\hline 0.57 & $170^{*}$ & 100 & 1.5 & 304 & 3 & VII & $\begin{array}{l}\text { With Chemically } \\
\text { Sacrificial Zirconium }\end{array}$ \\
\hline 0.56 & $160^{*}$ & 100 & 1.5 & 304 & 3 & VII & $\begin{array}{l}\text { With Chemically } \\
\text { Sacrificial Zirconium }\end{array}$ \\
\hline 0.84 & $250^{*}$ & 100 & 1.5 & 304 & 5 & VII & $\begin{array}{l}\text { With Chemically } \\
\text { Sacrificial Zirconium }\end{array}$ \\
\hline 0.86 & $250^{*}$ & 100 & 1.5 & 304 & 5 & VII & $\begin{array}{l}\text { With Chemically } \\
\text { Sacrificial Zirconium }\end{array}$ \\
\hline 1.19 & $350^{*}$ & 100 & 1.5 & 304 & 9 & VII & $\begin{array}{l}\text { With Chemically } \\
\text { Sacrificial Zirconium }\end{array}$ \\
\hline 1.26 & $370^{*}$ & 100 & 1.5 & 304 & 9 & VII & $\begin{array}{l}\text { With Chemically } \\
\text { Sacrificial Zirconium }\end{array}$ \\
\hline 6.14 & $1,810^{*}$ & Ambient & 1.5 & 304 & 13 & VIII & \\
\hline 5.99 & $1,760^{*}$ & Ambient & 1.5 & 304 & 13 & VIII & \\
\hline 2.34 & $688^{*}$ & Ambient & 1.5 & 304 & 13 & VIII & $\begin{array}{l}\text { With Chemically } \\
\text { Sacrificial Zirconium }\end{array}$ \\
\hline 2.21 & $650^{*}$ & Ambient & 1.5 & 304 & 13 & VIII & $\begin{array}{l}\text { With Chemically } \\
\text { Sacrificial Zirconium }\end{array}$ \\
\hline 3.6 & $* 1,100$ & 50 & 1.5 & 304 & 10 & VIII & $\begin{array}{l}\text { With Chemically } \\
\text { Sacrificial Zirconium }\end{array}$ \\
\hline 3.19 & $938^{*}$ & 50 & 1.5 & 304 & 10 & VIII & $\begin{array}{l}\text { With Chemically } \\
\text { Sacrificial Zirconium }\end{array}$ \\
\hline 0.09 & $30^{*}$ & 100 & 0.3 & 304 & 2 & VII & \\
\hline 0.1 & $30^{*}$ & 100 & 0.3 & 304 & 2 & VII & \\
\hline 0.05 & $10^{*}$ & 100 & 0.3 & 304 & 3 & VII & \\
\hline 0.06 & $20^{*}$ & 100 & 0.3 & 304 & 3 & VII & \\
\hline 0.12 & $35^{*}$ & 100 & 0.3 & 304 & 5 & VII & \\
\hline 0.11 & $32^{*}$ & 100 & 0.3 & 304 & 5 & VII & \\
\hline 0.08 & $20^{*}$ & 100 & 0.3 & 304 & 9 & VII & \\
\hline 0.06 & $20^{*}$ & 100 & 0.3 & 304 & 9 & VII & \\
\hline 0.2 & $60^{*}$ & 100 & 0.3 & 304 & 2 & VII & $\begin{array}{l}\text { Stainless Steel Type } \\
304 \text { Welded with ER- } \\
310 \text { Rod }\end{array}$ \\
\hline 0.18 & $53^{*}$ & 100 & 0.3 & 304 & 2 & VII & $\begin{array}{l}\text { Stainless Steel Type } \\
304 \text { Welded with ER- } \\
310 \text { Rod }\end{array}$ \\
\hline
\end{tabular}


Table 4-11. Corrosion of Borated Stainless Steel Type 304L for Use as "Saltwater" Rates (Continued)

\begin{tabular}{|c|c|c|c|c|c|c|c|}
\hline $\begin{array}{c}\text { Corrosion Rate } \\
\text { (mils/month) }\end{array}$ & $\begin{array}{c}\text { Corrosion Rate } \\
(\mu \mathrm{m} / \mathrm{yr})\end{array}$ & $\begin{array}{c}\text { Temp } \\
\left({ }^{\circ} \mathrm{C}\right) \\
\end{array}$ & $\begin{array}{c}\text { Wt. \% } \\
\text { B }\end{array}$ & $\begin{array}{l}\text { Steel } \\
\text { Type }\end{array}$ & $\begin{array}{c}\text { Solution } \\
\text { Type }\end{array}$ & $\begin{array}{l}\text { Table in } \\
\text { Source }\end{array}$ & Other Comments \\
\hline 0.06 & $20^{*}$ & 100 & 0.3 & 304 & 3 & VII & $\begin{array}{l}\text { Stainless Steel Type } \\
304 \text { Welded with ER- } \\
310 \text { Rod }\end{array}$ \\
\hline 0.08 & $20^{*}$ & 100 & 0.3 & 304 & 3 & VII & $\begin{array}{l}\text { Stainless Steel Type } \\
304 \text { Welded with ER- } \\
310 \text { Rod }\end{array}$ \\
\hline 0.09 & $30 *$ & 100 & 0.3 & 304 & 5 & VII & $\begin{array}{l}\text { Stainless Steel Type } \\
304 \text { Welded with ER- } \\
310 \text { Rod }\end{array}$ \\
\hline 0.1 & *30 & 100 & 0.3 & 304 & 5 & VII & $\begin{array}{l}\text { Stainless Steel Type } \\
304 \text { Welded with ER- } \\
310 \text { Rod }\end{array}$ \\
\hline 0.11 & $32^{*}$ & 100 & 0.3 & 304 & 9 & VII & $\begin{array}{l}\text { Stainless Steel Type } \\
304 \text { Welded with ER- } \\
310 \text { Rod }\end{array}$ \\
\hline 0.09 & $30^{*}$ & 100 & 0.3 & 304 & 9 & VII & $\begin{array}{l}\text { Stainless Steel Type } \\
304 \text { Welded with ER- } \\
310 \text { Rod }\end{array}$ \\
\hline 0.12 & $35^{*}$ & 100 & 0.3 & 304 & 2 & VII & $\begin{array}{l}\text { Stainless Steel Type } \\
304 \text { Welded to } \\
\text { Stainless Steel Type } \\
304 \text { with ER-310 Rod }\end{array}$ \\
\hline 0.13 & $38^{*}$ & 100 & 0.3 & 304 & 2 & VII & $\begin{array}{l}\text { Stainless Steel Type } \\
304 \text { Welded to } \\
\text { Stainless Steel Type } \\
304 \text { with ER-310 Rod }\end{array}$ \\
\hline 0.04 & $10^{*}$ & 100 & 0.3 & 304 & 3 & VII & $\begin{array}{l}\text { Stainless Steel Type } \\
304 \text { Welded to } \\
\text { Stainless Steel Type } \\
304 \text { with ER-310 Rod }\end{array}$ \\
\hline 0.05 & $10^{*}$ & 100 & 0.3 & 304 & 3 & VII & $\begin{array}{l}\text { Stainless Steel Type } \\
304 \text { Welded to } \\
\text { Stainless Steel Type } \\
304 \text { with ER-310 Rod }\end{array}$ \\
\hline 0.19 & $56^{*}$ & 100 & 0.3 & 304 & 5 & VII & $\begin{array}{l}\text { Stainless Steel Type } \\
304 \text { Welded to } \\
\text { Stainless Steel Type } \\
304 \text { with ER-310 Rod }\end{array}$ \\
\hline 0.12 & $35^{*}$ & 100 & 0.3 & 304 & 5 & VII & $\begin{array}{l}\text { Stainless Steel Type } \\
304 \text { Welded to } \\
\text { Stainless Steel Type } \\
304 \text { with ER-310 Rod }\end{array}$ \\
\hline 0.09 & $26.46^{*}$ & 100 & 0.3 & 304 & 9 & VII & $\begin{array}{l}\text { Stainless Steel Type } \\
304 \text { Welded to } \\
\text { Stainless Steel Type } \\
304 \text { with ER-310 Rod }\end{array}$ \\
\hline 0.11 & $32.34^{*}$ & 100 & 0.3 & 304 & 9 & VII & $\begin{array}{l}\text { Stainless Steel Type } \\
304 \text { Welded to } \\
\text { Stainless Steel Type } \\
304 \text { with ER-310 Rod }\end{array}$ \\
\hline 0.01 & $3^{*}$ & Ambient & 0.3 & 304 & 10 & VIII & \\
\hline 0.01 & $3^{*}$ & Ambient & 0.3 & 304 & 10 & VIII & \\
\hline 0.02 & $6^{*}$ & Ambient & 0.3 & 304 & 13 & VIII & \\
\hline 0.07 & $20^{*}$ & Ambient & 0.3 & 304 & 13 & VIII & \\
\hline
\end{tabular}


Table 4-11. Corrosion of Borated Stainless Steel Type 304L for Use as "Saltwater" Rates (Continued)

\begin{tabular}{|c|c|c|c|c|c|c|c|}
\hline $\begin{array}{c}\text { Corrosion Rate } \\
\text { (mils/month) }\end{array}$ & $\begin{array}{c}\text { Corrosion Rate } \\
(\mu \mathrm{m} / \mathrm{yr})\end{array}$ & $\begin{array}{c}\text { Temp } \\
\left({ }^{\circ} \mathrm{C}\right)\end{array}$ & $\begin{array}{c}\text { Wt. \% } \\
\text { B }\end{array}$ & $\begin{array}{l}\text { Steel } \\
\text { Type }\end{array}$ & $\begin{array}{c}\text { Solution } \\
\text { Type }\end{array}$ & $\begin{array}{l}\text { Table in } \\
\text { Source }\end{array}$ & Other Comments \\
\hline 0.09 & $30^{*}$ & Ambient & 0.3 & 304 & 10 & VIII & $\begin{array}{l}\text { Stainless Steel Type } \\
304 \text { Welded with } \\
\text { Stainless Steel Type } \\
\text { ER-310 Rod }\end{array}$ \\
\hline 0.08 & $20^{*}$ & Ambient & 0.3 & 304 & 10 & VIII & $\begin{array}{l}\text { Stainless Steel Type } \\
304 \text { Welded with } \\
\text { Stainless Steel Type } \\
\text { ER-310 Rod }\end{array}$ \\
\hline 0.08 & $20^{*}$ & Ambient & 0.3 & 304 & 13 & VIII & $\begin{array}{l}\text { Stainless Steel Type } \\
304 \text { Welded with } \\
\text { Stainless Steel Type } \\
\text { ER-310 Rod }\end{array}$ \\
\hline 0.06 & $20^{*}$ & Ambient & 0.3 & 304 & 13 & VIII & $\begin{array}{l}\text { Stainless Steel Type } \\
304 \text { Welded with } \\
\text { Stainless Steel Type } \\
\text { ER-310 Rod }\end{array}$ \\
\hline 0.09 & $30^{*}$ & Ambient & 0.3 & 304 & 10 & VIII & $\begin{array}{l}\text { Stainless Steel Type } \\
304 \text { Welded to } \\
\text { Stainless Steel Type } \\
304 \text { with ER-310 Rod }\end{array}$ \\
\hline 0.07 & $20^{*}$ & Ambient & 0.3 & 304 & 10 & VIII & $\begin{array}{l}\text { Stainless Steel Type } \\
304 \text { Welded to } \\
\text { Stainless Steel Type } \\
304 \text { with ER-310 Rod }\end{array}$ \\
\hline 0.33 & $97^{*}$ & 50 & 0.3 & 304 & 10 & VIII & \\
\hline 0.22 & $65^{*}$ & 50 & 0.3 & 304 & 10 & VIII & \\
\hline 0.14 & $41^{*}$ & 50 & 0.3 & 304 & 13 & VIII & \\
\hline 0.13 & $38^{*}$ & 50 & 0.3 & 304 & 13 & VIII & \\
\hline 0.47 & $140^{*}$ & 50 & 0.3 & 304 & 10 & VIII & $\begin{array}{l}\text { Stainless Steel Type } \\
304 \text { Welded with ER- } \\
310 \text { Rod }\end{array}$ \\
\hline 0.46 & $140^{*}$ & 50 & 0.3 & 304 & 10 & VIII & $\begin{array}{l}\text { Stainless Steel Type } \\
304 \text { Welded with ER- } \\
310 \text { Rod }\end{array}$ \\
\hline 0.27 & $79^{*}$ & 50 & 0.3 & 304 & 13 & VIII & $\begin{array}{l}\text { Stainless Steel Type } \\
304 \text { Welded with ER- } \\
310 \text { Rod }\end{array}$ \\
\hline 0.22 & $65^{*}$ & 50 & 0.3 & 304 & 13 & VIII & $\begin{array}{l}\text { Stainless Steel Type } \\
304 \text { Welded with ER- } \\
310 \text { Rod }\end{array}$ \\
\hline 0.48 & $140^{*}$ & 50 & 0.3 & 304 & 10 & VIII & $\begin{array}{l}\text { Stainless Steel Type } \\
304 \text { Welded to } \\
\text { Stainless Steel Type } \\
304 \text { with ER-310 Rod }\end{array}$ \\
\hline 0.5 & $100^{*}$ & 50 & 0.3 & 304 & 10 & VIII & $\begin{array}{l}\text { Stainless Steel Type } \\
304 \text { Welded to } \\
\text { Stainless Steel Type } \\
304 \text { with ER-310 Rod }\end{array}$ \\
\hline
\end{tabular}

Source: Cole 1976 [DIRS 159369].

NOTE: For more information on the solution type, see aqueous-B-Gd-steels.xls in Appendix II.

Values marked with an asterisk $\left({ }^{*}\right)$ calculated in aqueous- $B-G d-s t e e / s . x / s$ in Appendix II.

$1 \mathrm{mil}=25.4 \mu \mathrm{m}$. 
Table 4-12. Corrosion Rates of Gadolinium-Alloyed Steel

\begin{tabular}{|c|c|c|c|c|c|c|}
\hline $\begin{array}{c}\text { Corrosion Rate } \\
\text { (mils/month) }\end{array}$ & $\begin{array}{c}\text { Corrosion Rate } \\
(\mu \mathrm{m} / \mathrm{yr})\end{array}$ & Temp $\left({ }^{\circ} \mathrm{C}\right)$ & $\%$ Gd & $\begin{array}{l}\text { Steel } \\
\text { Type }\end{array}$ & $\begin{array}{c}\text { Solution } \\
\text { Type }\end{array}$ & $\begin{array}{l}\text { Table in } \\
\text { Source }\end{array}$ \\
\hline \multicolumn{7}{|c|}{ "Freshwater" } \\
\hline 0.03 & $9^{*}$ & 100 & 0.3 & 304 & 6 & VII \\
\hline 0.03 & $9^{*}$ & 100 & 0.3 & 304 & 6 & VII \\
\hline 0.01 & $3^{*}$ & Ambient & 0.3 & 304 & 11 & VIII \\
\hline 0.01 & $3^{*}$ & Ambient & 0.3 & 304 & 11 & VIII \\
\hline 0.05 & $20^{*}$ & 50 & 0.3 & 304 & 11 & VIII \\
\hline 0.01 & $3^{*}$ & 50 & 0.3 & 304 & 11 & VIII \\
\hline \multicolumn{7}{|c|}{ "Harshwater" } \\
\hline 0.03 & 9 & 100 & 0.3 & 304 & 2 & VII \\
\hline 0.04 & 10 & 100 & 0.3 & 304 & 2 & VII \\
\hline 0.02 & 6 & 100 & 0.3 & 304 & 3 & VII \\
\hline 0.02 & 6 & 100 & 0.3 & 304 & 3 & VII \\
\hline 0.07 & 20 & 100 & 0.3 & 304 & 5 & VII \\
\hline 0.08 & 20 & 100 & 0.3 & 304 & 5 & VII \\
\hline 0.02 & 6 & 100 & 0.3 & 304 & 9 & VII \\
\hline 0.02 & 6 & 100 & 0.3 & 304 & 9 & VII \\
\hline 0.01 & 3 & Ambient & 0.3 & 304 & 10 & VIII \\
\hline 0.01 & 3 & Ambient & 0.3 & 304 & 10 & VIII \\
\hline 0.01 & 3 & Ambient & 0.3 & 304 & 13 & VIII \\
\hline 0.01 & 3 & Ambient & 0.3 & 304 & 13 & VIII \\
\hline 1.04 & 306 & 50 & 0.3 & 304 & 10 & VIII \\
\hline 1.02 & 300 & 50 & 0.3 & 304 & 10 & VIII \\
\hline 0.26 & 76 & 50 & 0.3 & 304 & 13 & VIII \\
\hline 0.31 & 91 & 50 & 0.3 & 304 & 13 & VIII \\
\hline
\end{tabular}

Source: Cole 1976 [DIRS 159369]

NOTES: For more information on the solution type, see aqueous-B-Gd-steels.x/s in Appendix II. Values marked with an asterisk (*) calculated in aqueous- $B-G d-$ steels. $x / \mathrm{s}$ "in Appendix II. 1 mil $=25.4 \mu \mathrm{m}$.

\subsubsection{Carbon Steel Type A516}

The rates used to describe the corrosion of Carbon Steel Type A516 for long-term studies ( $>1$ year) are found in Table 4-13 through Table 4-16. Further explanation on the use of these rates can be found in Section 6.2.3.

The rates used to describe the corrosion of Carbon Steel Type A516 for short-term studies ( $\leq 0.53$ years) are found in Table 4-17 through Table 4-20. Further explanation on the use of these rates can be found in Section 6.2.3. 
Table 4-13. Carbon Steel Corrosion at 1 Year in Freshwater at $90^{\circ} \mathrm{C}$

\begin{tabular}{|c|c|c|c|c|}
\hline $\begin{array}{c}\text { Corrosion Rate } \\
(\mu \mathrm{m} / \mathrm{yr})\end{array}$ & $\begin{array}{l}\text { Steel } \\
\text { Type }\end{array}$ & Table in Source & $\begin{array}{l}\text { Type of } \\
\text { Analysis }\end{array}$ & Fluid Type \\
\hline 43.65 & A516 & Sec. 2.2.6, Sup. 1 , p. $2.2-40$ & WL & SDW J-13 Well Water \\
\hline 45.19 & A516 & Sec. 2.2.6, Sup. 1, p. 2.2-40 & WL & SDW J-13 Well Water \\
\hline 48.30 & A516 & Sec. 2.2 .6 , Sup. 1 , p. $2.2-40$ & WL & SDW J-13 Well Water \\
\hline 55.97 & A516 & Sec. 2.2.6, Sup. 1, p. 2.2-41 & WL & SDW J-13 Well Water \\
\hline 63.58 & A516 & Sec. 2.2.6, Sup. 1, p. 2.2-41 & WL & SDW J-13 Well Water \\
\hline 50.17 & A516 & Sec. 2.2.6, Sup. 1, p. 2.2-41 & WL & SDW J-13 Well Water \\
\hline 42.42 & A516 & Sec. 2.2.6, Sup. 2, p. 2.2-96 & WL & SDW J-13 Well Water \\
\hline 45.91 & A516 & Sec. 2.2.6, Sup. 2, p. 2.2-96 & WL & SDW J-13 Well Water \\
\hline 29.53 & A516 & Sec. 2.2.6, Sup. 2, p. 2.2-96 & WL & SDW J-13 Well Water \\
\hline 65.04 & A516 & Sec. 2.2.6, Sup. 2, p. 2.2-97 & WL & SDW J-13 Well Water \\
\hline 65.73 & A516 & Sec. 2.2.6, Sup. 2, p. 2.2-97 & WL & SDW J-13 Well Water \\
\hline 88.68 & A516 & Sec. 2.2.6, Sup. 2, p. 2.2-98 & WL & SDW J-13 Well Water \\
\hline 46.79 & A27 & Sec. 2.2 .6 , Sup. 1 , p. $2.2-43$ & WL & SDW J-13 Well Water \\
\hline 68.91 & A27 & Sec. 2.2.6, Sup. 1, p. 2.2-43 & WL & SDW J-13 Well Water \\
\hline 46.72 & A27 & Sec. 2.2.6, Sup. 1, p. 2.2-43 & WL & SDW J-13 Well Water \\
\hline 46.31 & A27 & Sec. 2.2.6, Sup. 1, p. 2.2-44 & WL & SDW J-13 Well Water \\
\hline 36.38 & A27 & Sec. 2.2.6, Sup. 1, p. 2.2-44 & WL & SDW J-13 Well Water \\
\hline 65.88 & A27 & Sec. 2.2.6, Sup. 1, p. 2.2-44 & WL & SDW J-13 Well Water \\
\hline 58.48 & $\mathrm{~A} 27$ & Sec. 2.2.6, Sup. 2, p. 2.2-99 & WL & SDW J-13 Well Water \\
\hline 35.87 & A27 & Sec. 2.2.6, Sup. 2, p. 2.2-99 & WL & SDW J-13 Well Water \\
\hline 50.14 & $\mathrm{~A} 27$ & Sec. 2.2.6, Sup. 2, p. 2.2-99 & WL & SDW J-13 Well Water \\
\hline 49.10 & A27 & Sec. 2.2.6, Sup. 2, p. 2.2-100 & WL & SDW J-13 Well Water \\
\hline 53.98 & $\mathrm{~A} 27$ & Sec. 2.2.6, Sup. 2 , p. $2.2-100$ & WL & SDW J-13 Well Water \\
\hline 40.46 & A27 & Sec. 2.2.6, Sup. 2, p. $2.2-100$ & WL & SDW J-13 Well Water \\
\hline
\end{tabular}

Source: McCright 1998 [DIRS 114637].

NOTE: $W L=$ weight loss; SDW = simulated dilute well. 
Table 4-14. Carbon Steel Corrosion at 1 Year in Saltwater at $90^{\circ} \mathrm{C}$

\begin{tabular}{|c|c|c|c|c|}
\hline $\begin{array}{c}\text { Corrosion } \\
\text { Rate } \\
(\mu \mathrm{m} / \mathrm{yr})\end{array}$ & $\begin{array}{l}\text { Steel } \\
\text { Type }\end{array}$ & Table in Source & $\begin{array}{c}\text { Type of } \\
\text { Analysis }\end{array}$ & Fluid Type \\
\hline 6.96 & A516 & Sec. 2.2.6, Sup. 1, p. 2.2-40 & WL & SCW J-13 Well Water \\
\hline 5.75 & A516 & Sec. 2.2.6, Sup. 1, p. $2.2-41$ & WL & SCW J-13 Well Water \\
\hline 3.69 & A516 & Sec. 2.2.6, Sup. 1, p. $2.2-41$ & WL & SCW J-13 Well Water \\
\hline 9.35 & A516 & Sec. 2.2.6, Sup. 1, p. 2.2-42 & WL & SCW J-13 Well Water \\
\hline 6.46 & A516 & Sec. 2.2.6, Sup. 1, p. 2.2-42 & WL & SCW J-13 Well Water \\
\hline 6.85 & A516 & Sec. 2.2.6, Sup. 1, p. 2.2-42 & WL & SCW J-13 Well Water \\
\hline 7.69 & A516 & Sec. 2.2.6, Sup. 2, p. 2.2-97 & WL & SCW J-13 Well Water \\
\hline 6.53 & A516 & Sec. 2.2.6, Sup. 2, p. 2.2-97 & WL & SCW J-13 Well Water \\
\hline 5.16 & A516 & Sec. 2.2.6, Sup. 2, p. 2.2-97 & WL & SCW J-13 Well Water \\
\hline 6.64 & A516 & Sec. 2.2.6, Sup. 2, p. 2.2-98 & WL & SCW J-13 Well Water \\
\hline 6.01 & A516 & Sec. 2.2.6, Sup. 2, p. 2.2-98 & WL & SCW J-13 Well Water \\
\hline 6.25 & A516 & Sec. 2.2.6, Sup. 2, p. 2.2-98 & WL & SCW J-13 Well Water \\
\hline 6.51 & $\mathrm{~A} 27$ & Sec. 2.2 .6 , Sup. 1 , p. 2.2-43 & WL & SCW J-13 Well Water \\
\hline 7.13 & $\mathrm{~A} 27$ & Sec. 2.2.6, Sup. 1 , p. $2.2-43$ & WL & SCW J-13 Well Water \\
\hline 5.32 & $\mathrm{~A} 27$ & Sec. 2.2.6, Sup. 1, p. $2.2-43$ & WL & SCW J-13 Well Water \\
\hline 6.13 & A27 & Sec. 2.2.6, Sup. 1, p. 2.2-45 & WL & SCW J-13 Well Water \\
\hline 5.91 & A27 & Sec. 2.2.6, Sup. 1, p. 2.2-45 & WL & SCW J-13 Well Water \\
\hline 8.20 & $\mathrm{~A} 27$ & Sec. 2.2.6, Sup. 1, p. 2.2-45 & WL & SCW J-13 Well Water \\
\hline 8.40 & A27 & Sec. 2.2.6, Sup. 2, p. 2.2-99 & WL & SCW J-13 Well Water \\
\hline 7.84 & $\mathrm{~A} 27$ & Sec. 2.2.6, Sup. 2, p. 2.2-99 & WL & SCW J-13 Well Water \\
\hline 8.24 & $\mathrm{~A} 27$ & Sec. 2.2.6, Sup. 2, p. 2.2-99 & WL & SCW J-13 Well Water \\
\hline 7.87 & $\mathrm{~A} 27$ & Sec. 2.2.6, Sup. 2 , p. $2.2-100$ & WL & SCW J-13 Well Water \\
\hline 8.04 & $\mathrm{~A} 27$ & Sec. 2.2.6, Sup. 2 , p. $2.2-100$ & WL & SCW J-13 Well Water \\
\hline 7.32 & $\mathrm{~A} 27$ & Sec. 2.2.6, Sup. 2 , p. $2.2-100$ & WL & SCW J-13 Well Water \\
\hline
\end{tabular}

Source: McCright 1998 [DIRS 114637].

NOTE: $\mathrm{WL}=$ weight loss; $\mathrm{SCW}=$ simulated concentrated well. 
Table 4-15. Carbon Steel Corrosion at 1 Year in Freshwater at $60^{\circ} \mathrm{C}$

\begin{tabular}{|c|c|c|c|c|}
\hline $\begin{array}{c}\text { Corrosion } \\
\text { Rate }(\mu \mathrm{m} / \mathrm{yr})\end{array}$ & $\begin{array}{l}\text { Steel } \\
\text { Type }\end{array}$ & Table in Source & $\begin{array}{l}\text { Type of } \\
\text { Analysis }\end{array}$ & Fluid Type \\
\hline 80.00 & A516 & Sec. 2.2.6, Sup. 1, p. 2.2-39 & WL & SDW J-13 Well Water \\
\hline 66.75 & $\mathrm{~A} 516$ & Sec. 2.2.6, Sup. 1, p. 2.2-39 & WL & SDW J-13 Well Water \\
\hline 77.31 & A516 & Sec. 2.2.6, Sup. 1, p. 2.2-39 & WL & SDW J-13 Well Water \\
\hline 69.84 & A516 & Sec. 2.2.6, Sup. 1, p. 2.2-41 & WL & SDW J-13 Well Water \\
\hline 83.74 & A516 & Sec. 2.2.6, Sup. 1, p. 2.2-41 & WL & SDW J-13 Well Water \\
\hline 79.29 & A516 & Sec. 2.2.6, Sup. 1, p. 2.2-41 & WL & SDW J-13 Well Water \\
\hline 71.25 & A516 & Sec. 2.2.6, Sup. 2, p. 2.2-96 & WL & SDW J-13 Well Water \\
\hline 65.77 & A516 & Sec. 2.2.6, Sup. 2, p. 2.2-96 & WL & SDW J-13 Well Water \\
\hline 70.00 & A516 & Sec. 2.2.6, Sup. 2, p. 2.2-96 & WL & SDW J-13 Well Water \\
\hline 72.64 & A516 & Sec. 2.2.6, Sup. 2, p. 2.2-97 & WL & SDW J-13 Well Water \\
\hline 72.89 & A516 & Sec. 2.2.6, Sup. 2, p. 2.2-97 & WL & SDW J-13 Well Water \\
\hline 106.93 & A516 & Sec. 2.2.6, Sup. 2, p. 2.2-97 & WL & SDW J-13 Well Water \\
\hline 90.97 & A27 & Sec. 2.2.6, Sup. 1, p. 2.2-42 & WL & SDW J-13 Well Water \\
\hline 83.26 & A27 & Sec. 2.2.6, Sup. 1, p. 2.2-42 & WL & SDW J-13 Well Water \\
\hline 74.29 & A27 & Sec. 2.2.6, Sup. 1, p. 2.2-42 & WL & SDW J-13 Well Water \\
\hline 72.87 & A27 & Sec. 2.2.6, Sup. 1, p. 2.2-44 & WL & SDW J-13 Well Water \\
\hline 73.47 & A27 & Sec. 2.2.6, Sup. 1, p. 2.2-44 & WL & SDW J-13 Well Water \\
\hline 83.66 & A27 & Sec. 2.2.6, Sup. 1, p. 2.2-44 & WL & SDW J-13 Well Water \\
\hline 74.51 & A27 & Sec. 2.2.6, Sup. 2, p. 2.2-98 & WL & SDW J-13 Well Water \\
\hline 74.60 & A27 & Sec. 2.2.6, Sup. 2, p. 2.2-98 & WL & SDW J-13 Well Water \\
\hline 75.41 & $\mathrm{~A} 27$ & Sec. 2.2.6, Sup. 2, p. 2.2-98 & WL & SDW J-13 Well Water \\
\hline 72.21 & $\mathrm{~A} 27$ & Sec. 2.2.6, Sup. 2, p. $2.2-100$ & WL & SDW J-13 Well Water \\
\hline 80.87 & $\mathrm{~A} 27$ & Sec. 2.2.6, Sup. 2, p. $2.2-100$ & WL & SDW J-13 Well Water \\
\hline 85.68 & A27 & Sec. 2.2 .6 , Sup. 2 , p. $2.2-100$ & WL & SDW J-13 Well Water \\
\hline
\end{tabular}

Source: McCright 1998 [DIRS 114637].

NOTE: $\mathrm{WL}=$ weight loss; SDW = simulated dilute well. 
Table 4-16. Carbon Steel Corrosion at 1 Year in Saltwater at $60^{\circ} \mathrm{C}$

\begin{tabular}{|c|c|c|c|c|}
\hline $\begin{array}{c}\text { Corrosion } \\
\text { Rate } \\
(\mu \mathrm{m} / \mathrm{yr})\end{array}$ & $\begin{array}{l}\text { Steel } \\
\text { Type }\end{array}$ & Table in Source & $\begin{array}{c}\text { Type of } \\
\text { Analysis }\end{array}$ & Fluid Type \\
\hline 7.93 & A516 & Sec. 2.2.6, Sup. 1, p. $2.2-40$ & WL & SCW J-13 Well Water \\
\hline 11.19 & A516 & Sec. 2.2 .6 , Sup. 1 , p. $2.2-40$ & WL & SCW J-13 Well Water \\
\hline 8.03 & A516 & Sec. 2.2.6, Sup. 1, p. 2.2-40 & WL & SCW J-13 Well Water \\
\hline 9.45 & A516 & Sec. 2.2.6, Sup. 1, p. 2.2-41 & WL & SCW J-13 Well Water \\
\hline 11.05 & A516 & Sec. 2.2.6, Sup. 1 , p. 2.2-42 & WL & SCW J-13 Well Water \\
\hline 9.1 & A516 & Sec. 2.2.6, Sup. 1, p. $2.2-42$ & WL & SCW J-13 Well Water \\
\hline 6.77 & A516 & Sec. 2.2.6, Sup. 2, p. 2.2-97 & WL & SCW J-13 Well Water \\
\hline 10.07 & A516 & Sec. 2.2.6, Sup. 2, p. 2.2-97 & WL & SCW J-13 Well Water \\
\hline 11.72 & A516 & Sec. 2.2.6, Sup. 2, p. 2.2-97 & WL & SCW J-13 Well Water \\
\hline 12.2 & A516 & Sec. 2.2.6, Sup. 2, p. 2.2-98 & WL & SCW J-13 Well Water \\
\hline 12.9 & A516 & Sec. 2.2.6, Sup. 2, p. 2.2-98 & WL & SCW J-13 Well Water \\
\hline 12.99 & A516 & Sec. 2.2.6, Sup. 2, p. 2.2-98 & WL & SCW J-13 Well Water \\
\hline 10.67 & $\mathrm{~A} 27$ & Sec. 2.2.6, Sup. 1, p. $2.2-43$ & WL & SCW J-13 Well Water \\
\hline 10.00 & $\mathrm{~A} 27$ & Sec. 2.2.6, Sup. 1, p. $2.2-43$ & WL & SCW J-13 Well Water \\
\hline 7.42 & $\mathrm{~A} 27$ & Sec. 2.2 .6 , Sup. 1 , p. $2.2-43$ & WL & SCW J-13 Well Water \\
\hline 8.09 & A27 & Sec. 2.2.6, Sup. 1, p. 2.2-44 & WL & SCW J-13 Well Water \\
\hline 10.32 & $\mathrm{~A} 27$ & Sec. 2.2.6, Sup. 1, p. 2.2-44 & WL & SCW J-13 Well Water \\
\hline 10.83 & A27 & Sec. 2.2.6, Sup. 1, p. 2.2-44 & WL & SCW J-13 Well Water \\
\hline 12.09 & $\mathrm{~A} 27$ & Sec. 2.2.6, Sup. 2, p. 2.2-99 & WL & SCW J-13 Well Water \\
\hline 14.36 & A27 & Sec. 2.2.6, Sup. 2, p. 2.2-99 & $W L$ & SCW J-13 Well Water \\
\hline 12.21 & A27 & Sec. 2.2.6, Sup. 2, p. $2.2-100$ & $W L$ & SCW J-13 Well Water \\
\hline 11.94 & A27 & Sec. 2.2.6, Sup. 2, p. $2.2-100$ & WL & SCW J-13 Well Water \\
\hline 12.62 & A27 & Sec. 2.2.6, Sup. 2, p. $2.2-100$ & WL & SCW J-13 Well Water \\
\hline
\end{tabular}

Source: McCright 1998 [DIRS 114637].

NOTE: $\mathrm{WL}=$ weight loss; $\mathrm{SCW}=$ simulated concentrated well. 
Table 4-17. Carbon Steel Corrosion at 0.53 Years in Freshwater at $90^{\circ} \mathrm{C}$

\begin{tabular}{|c|c|c|c|c|}
\hline $\begin{array}{c}\text { Corrosion Rate } \\
(\mu \mathrm{m} / \mathrm{yr})\end{array}$ & $\begin{array}{l}\text { Steel } \\
\text { Type }\end{array}$ & Table in Source & $\begin{array}{l}\text { Type of } \\
\text { Analysis }\end{array}$ & Fluid Type \\
\hline 89.41 & A516 & Sec. 2.2.6, Sup. 1, p. $2.2-40$ & WL & SDW J-13 Well Water \\
\hline 68.90 & A516 & Sec. 2.2.6, Sup. 1, p. $2.2-40$ & WL & SDW J-13 Well Water \\
\hline 84.02 & A516 & Sec. 2.2.6, Sup. 1 , p. $2.2-40$ & WL & SDW J-13 Well Water \\
\hline 87.65 & A516 & Sec. 2.2.6, Sup. 1, p. 2.2-41 & WL & SDW J-13 Well Water \\
\hline 107.46 & A516 & Sec. 2.2.6, Sup. 1, p. 2.2-41 & WL & SDW J-13 Well Water \\
\hline 130.02 & A516 & Sec. 2.2.6, Sup. 1, p. $2.2-41$ & $W L$ & SDW J-13 Well Water \\
\hline 76.96 & A516 & Sec. 2.2.6, Sup. 2, p. 2.2-96 & WL & SDW J-13 Well Water \\
\hline 70.45 & A516 & Sec. 2.2.6, Sup. 2, p. 2.2-96 & WL & SDW J-13 Well Water \\
\hline 74.29 & A516 & Sec. 2.2.6, Sup. 2, p. 2.2-96 & WL & SDW J-13 Well Water \\
\hline 180.42 & A516 & Sec. 2.2.6, Sup. 2, p. 2.2-97 & $W L$ & SDW J-13 Well Water \\
\hline 58.08 & A516 & Sec. 2.2.6, Sup. 2, p. 2.2-97 & WL & SDW J-13 Well Water \\
\hline 66.27 & A516 & Sec. 2.2.6, Sup. 2, p. 2.2-97 & WL & SDW J-13 Well Water \\
\hline 71.97 & $\mathrm{~A} 27$ & Sec. 2.2.6, Sup. 1 , p. $2.2-42$ & WL & SDW J-13 Well Water \\
\hline 79.61 & A27 & Sec. 2.2.6, Sup. 1, p. 2.2-42 & WL & SDW J-13 Well Water \\
\hline 97.43 & $\mathrm{~A} 27$ & Sec. 2.2.6, Sup. 1, p. 2.2-42 & WL & SDW J-13 Well Water \\
\hline 75.50 & A27 & Sec. 2.2.6, Sup. 1, p. 2.2-44 & WL & SDW J-13 Well Water \\
\hline 67.99 & $\mathrm{~A} 27$ & Sec. 2.2.6, Sup. 1, p. 2.2-44 & WL & SDW J-13 Well Water \\
\hline 89.88 & A27 & Sec. 2.2.6, Sup. 1, p. 2.2-44 & WL & SDW J-13 Well Water \\
\hline 78.85 & A27 & Sec. 2.2.6, Sup. 2, p. 2.2-99 & WL & SDW J-13 Well Water \\
\hline 85.06 & A27 & Sec. 2.2.6, Sup. 2, p. 2.2-99 & WL & SDW J-13 Well Water \\
\hline 79.56 & $\mathrm{~A} 27$ & Sec. 2.2.6, Sup. 2, p. 2.2-99 & $W L$ & SDW J-13 Well Water \\
\hline 74.56 & $\mathrm{~A} 27$ & Sec. 2.2.6, Sup. 2, p. 2.2-100 & WL & SDW J-13 Well Water \\
\hline 75.23 & $\mathrm{~A} 27$ & Sec. 2.2.6, Sup. 2, p. 2.2-100 & WL & SDW J-13 Well Water \\
\hline 77.05 & $\mathrm{~A} 27$ & Sec. 2.2.6, Sup. 2, p. $2.2-100$ & WL & SDW J-13 Well Water \\
\hline
\end{tabular}

Source: McCright 1998 [DIRS 114637].

NOTE: $\quad W L=$ weight loss; SDW = simulated dilute well. 
Table 4-18. Carbon Steel Corrosion at 0.5 Years in Saltwater at $90^{\circ} \mathrm{C}$

\begin{tabular}{|c|c|c|c|c|}
\hline $\begin{array}{c}\text { Corrosion } \\
\text { Rate } \\
(\mu \mathrm{m} / \mathrm{yr})\end{array}$ & $\begin{array}{l}\text { Steel } \\
\text { Type }\end{array}$ & Table in Source & $\begin{array}{l}\text { Type of } \\
\text { Analysis }\end{array}$ & Fluid Type \\
\hline 12.81 & A516 & Sec. 2.2.6, Sup. 1, p. 2.2-40 & WL & SCW J-13 Well Water \\
\hline 13.88 & A516 & Sec. 2.2.6, Sup. 1, p. $2.2-40$ & WL & SCW J-13 Well Water \\
\hline 10.92 & A516 & Sec. 2.2.6, Sup. 1 , p. $2.2-40$ & WL & SCW J-13 Well Water \\
\hline 19.08 & A516 & Sec. 2.2.6, Sup. 1, p. 2.2-42 & WL & SCW J-13 Well Water \\
\hline 14.01 & A516 & Sec. 2.2.6, Sup. 1, p. 2.2-42 & WL & SCW J-13 Well Water \\
\hline 16.2 & A516 & Sec. 2.2.6, Sup. 1 , p. 2.2-42 & WL & SCW J-13 Well Water \\
\hline 12.24 & A516 & Sec. 2.2.6, Sup. 2, p. 2.2-97 & WL & SCW J-13 Well Water \\
\hline 8.05 & A516 & Sec. 2.2.6, Sup. 2, p. 2.2-97 & WL & SCW J-13 Well Water \\
\hline 8.66 & A516 & Sec. 2.2.6, Sup. 2, p. 2.2-97 & WL & SCW J-13 Well Water \\
\hline 7.39 & A516 & Sec. 2.2.6, Sup. 2, p. 2.2-98 & WL & SCW J-13 Well Water \\
\hline 9.12 & A516 & Sec. 2.2.6, Sup. 2, p. 2.2-98 & WL & SCW J-13 Well Water \\
\hline 9.02 & A516 & Sec. 2.2.6, Sup. 2, p. 2.2-98 & WL & SCW J-13 Well Water \\
\hline 11.48 & $\mathrm{~A} 27$ & Sec. 2.2.6, Sup. 1, p. 2.2-43 & WL & SCW J-13 Well Water \\
\hline 13.67 & $\mathrm{~A} 27$ & Sec. 2.2.6, Sup. 1, p. $2.2-43$ & WL & SCW J-13 Well Water \\
\hline 9.03 & $\mathrm{~A} 27$ & Sec. 2.2.6, Sup. 1, p. $2.2-43$ & WL & SCW J-13 Well Water \\
\hline 16.84 & $\mathrm{~A} 27$ & Sec. 2.2.6, Sup. 1, p. 2.2-45 & WL & SCW J-13 Well Water \\
\hline 22.06 & $\mathrm{~A} 27$ & Sec. 2.2.6, Sup. 1 , p. $2.2-45$ & WL & SCW J-13 Well Water \\
\hline 18.06 & $\mathrm{~A} 27$ & Sec. 2.2.6, Sup. 1, p. 2.2-45 & WL & SCW J-13 Well Water \\
\hline 14.78 & A27 & Sec. 2.2.6, Sup. 2, p. 2.2-99 & WL & SCW J-13 Well Water \\
\hline 15.03 & $\mathrm{~A} 27$ & Sec. 2.2.6, Sup. 2, p. 2.2-99 & WL & SCW J-13 Well Water \\
\hline 12.60 & A27 & Sec. 2.2.6, Sup. 2, p. 2.2-99 & WL & SCW J-13 Well Water \\
\hline 9.74 & A27 & Sec. 2.2.6, Sup. 2, p. 2.2-100 & WL & SCW J-13 Well Water \\
\hline 10.46 & A27 & Sec. 2.2.6, Sup. 2, p. 2.2-100 & WL & SCW J-13 Well Water \\
\hline 11.47 & $\mathrm{~A} 27$ & Sec. 2.2 .6 , Sup. 2 , p. $2.2-100$ & WL & SCW J-13 Well Water \\
\hline
\end{tabular}

Source: McCright 1998 [DIRS 114637].

NOTE: $\quad W L=$ weight loss; $S C W=$ simulated concentrated well. 
Table 4-19. Carbon Steel Corrosion at 0.52 Years in Freshwater at $60^{\circ} \mathrm{C}$

\begin{tabular}{|c|c|c|c|c|}
\hline $\begin{array}{c}\text { Corrosion } \\
\text { Rate }(\mu \mathrm{m} / \mathrm{yr})\end{array}$ & $\begin{array}{l}\text { Steel } \\
\text { Type }\end{array}$ & Table in Source & $\begin{array}{l}\text { Type of } \\
\text { Analysis }\end{array}$ & Fluid Type \\
\hline 101.01 & A516 & Sec. 2.2.6, Sup. 1, p. 2.2-39 & WL & SDW J-13 Well Water \\
\hline 108.43 & $\mathrm{~A} 516$ & Sec. 2.2.6, Sup. 1, p. 2.2-39 & WL & SDW J-13 Well Water \\
\hline 105.33 & A516 & Sec. 2.2.6, Sup. 1, p. 2.2-39 & WL & SDW J-13 Well Water \\
\hline 88.15 & A516 & Sec. 2.2.6, Sup. 1, p. 2.2-41 & WL & SDW J-13 Well Water \\
\hline 101.95 & A516 & Sec. 2.2.6, Sup. 1, p. $2.2-41$ & WL & SDW J-13 Well Water \\
\hline 117.92 & A516 & Sec. 2.2.6, Sup. 1, p. $2.2-41$ & WL & SDW J-13 Well Water \\
\hline 87.80 & A516 & Sec. 2.2.6, Sup. 2, p. 2.2-96 & WL & SDW J-13 Well Water \\
\hline 88.29 & A516 & Sec. 2.2.6, Sup. 2, p. 2.2-96 & WL & SDW J-13 Well Water \\
\hline 78.71 & A516 & Sec. 2.2.6, Sup. 2, p. 2.2-96 & WL & SDW J-13 Well Water \\
\hline 112.46 & A516 & Sec. 2.2.6, Sup. 2, p. 2.2-97 & WL & SDW J-13 Well Water \\
\hline 103.18 & A516 & Sec. 2.2.6, Sup. 2, p. 2.2-97 & WL & SDW J-13 Well Water \\
\hline 99.73 & A516 & Sec. 2.2.6, Sup. 2 , p. 2.2-97 & WL & SDW J-13 Well Water \\
\hline 116.72 & A27 & Sec. 2.2.6, Sup. 1, p. 2.2-42 & WL & SDW J-13 Well Water \\
\hline 41.31 & A27 & Sec. 2.2.6, Sup. 1, p. 2.2-42 & WL & SDW J-13 Well Water \\
\hline 95.78 & A27 & Sec. 2.2.6, Sup. 1 , p. $2.2-42$ & WL & SDW J-13 Well Water \\
\hline 100.69 & A27 & Sec. 2.2 .6 , Sup. 1 , p. $2.2-43$ & WL & SDW J-13 Well Water \\
\hline 88.62 & A27 & Sec. 2.2 .6 , Sup. 1 , p. $2.2-43$ & WL & SDW J-13 Well Water \\
\hline 109.93 & A27 & Sec. 2.2.6, Sup. 1, p. 2.2-43 & WL & SDW J-13 Well Water \\
\hline 102.33 & A27 & Sec. 2.2.6, Sup. 2, p. 2.2-98 & WL & SDW J-13 Well Water \\
\hline 101.74 & A27 & Sec. 2.2.6, Sup. 2, p. 2.2-98 & WL & SDW J-13 Well Water \\
\hline 130.70 & $\mathrm{~A} 27$ & Sec. 2.2.6, Sup. 2, p. 2.2-98 & WL & SDW J-13 Well Water \\
\hline 108.44 & A27 & Sec. 2.2.6, Sup. 2, p. 2.2-99 & WL & SDW J-13 Well Water \\
\hline 92.90 & $\mathrm{~A} 27$ & Sec. 2.2.6, Sup. 2, p. 2.2-99 & WL & SDW J-13 Well Water \\
\hline 121.60 & A27 & Sec. 2.2.6, Sup. 2, p. 2.2-99 & WL & SDW J-13 Well Water \\
\hline
\end{tabular}

Source: McCright 1998 [DIRS 114637].

NOTE: $W L=$ weight loss; SDW = simulated dilute well. 
Table 4-20. Carbon Steel Corrosion at 0.50 Years in Saltwater at $60^{\circ} \mathrm{C}$

\begin{tabular}{|c|c|c|c|c|}
\hline $\begin{array}{c}\text { Corrosion } \\
\text { Rate } \\
(\mu \mathrm{m} / \mathrm{yr})\end{array}$ & $\begin{array}{l}\text { Steel } \\
\text { Type }\end{array}$ & Table in Source & $\begin{array}{l}\text { Type of } \\
\text { Analysis }\end{array}$ & Fluid Type \\
\hline 54.59 & A516 & Sec. 2.2.6, Sup. 1, p. $2.2-40$ & WL & SCW J-13 Well Water \\
\hline 54.97 & A516 & Sec. 2.2.6, Sup. 1, p. $2.2-40$ & WL & SCW J-13 Well Water \\
\hline 50.25 & A516 & Sec. 2.2.6, Sup. 1 , p. $2.2-40$ & WL & SCW J-13 Well Water \\
\hline 68.58 & A516 & Sec. 2.2.6, Sup. 1, p. 2.2-41 & WL & SCW J-13 Well Water \\
\hline 85.21 & A516 & Sec. 2.2.6, Sup. 1, p. 2.2-41 & WL & SCW J-13 Well Water \\
\hline 91.55 & A516 & Sec. 2.2.6, Sup. 1, p. 2.2-41 & WL & SCW J-13 Well Water \\
\hline 65.19 & A516 & Sec. 2.2.6, Sup. 2, p. 2.2-96 & WL & SCW J-13 Well Water \\
\hline 65.32 & A516 & Sec. 2.2.6, Sup. 2, p. 2.2-96 & WL & SCW J-13 Well Water \\
\hline 63.02 & A516 & Sec. 2.2.6, Sup. 2, p. 2.2-96 & WL & SCW J-13 Well Water \\
\hline 58.03 & A516 & Sec. 2.2.6, Sup. 2, p. 2.2-98 & WL & SCW J-13 Well Water \\
\hline 60.17 & A516 & Sec. 2.2.6, Sup. 2, p. 2.2-98 & WL & SCW J-13 Well Water \\
\hline 62.52 & A516 & Sec. 2.2.6, Sup. 2, p. 2.2-98 & WL & SCW J-13 Well Water \\
\hline 73.05 & $\mathrm{~A} 27$ & Sec. 2.2.6, Sup. 1, p. 2.2-43 & WL & SCW J-13 Well Water \\
\hline 78.39 & $\mathrm{~A} 27$ & Sec. 2.2.6, Sup. 1 , p. $2.2-43$ & WL & SCW J-13 Well Water \\
\hline 70.84 & $\mathrm{~A} 27$ & Sec. 2.2.6, Sup. 1 , p. $2.2-43$ & WL & SCW J-13 Well Water \\
\hline 53.66 & $\mathrm{~A} 27$ & Sec. 2.2.6, Sup. 1, p. 2.2-44 & WL & SCW J-13 Well Water \\
\hline 57.72 & $\mathrm{~A} 27$ & Sec. 2.2.6, Sup. 1, p. 2.2-44 & WL & SCW J-13 Well Water \\
\hline 55.38 & $\mathrm{~A} 27$ & Sec. 2.2.6, Sup. 1, p. 2.2-44 & WL & SCW J-13 Well Water \\
\hline 61.67 & A27 & Sec. 2.2.6, Sup. 2, p. 2.2-99 & WL & SCW J-13 Well Water \\
\hline 60.87 & $\mathrm{~A} 27$ & Sec. 2.2.6, Sup. 2, p. 2.2-99 & WL & SCW J-13 Well Water \\
\hline 62.14 & $\mathrm{~A} 27$ & Sec. 2.2.6, Sup. 2, p. 2.2-99 & WL & SCW J-13 Well Water \\
\hline 88.01 & A27 & Sec. 2.2.6, Sup. 2 , p. $2.2-100$ & WL & SCW J-13 Well Water \\
\hline 104.20 & $\mathrm{~A} 27$ & Sec. 2.2.6, Sup. 2 , p. $2.2-100$ & WL & SCW J-13 Well Water \\
\hline 88.57 & $\mathrm{~A} 27$ & Sec. 2.2.6, Sup. 2 , p. $2.2-100$ & WL & SCW J-13 Well Water \\
\hline
\end{tabular}

Source: McCright 1998 [DIRS 114637].

NOTE: $\mathrm{WL}=$ weight loss; SCW $=$ simulated concentrated well.

\subsubsection{Aluminum Alloys}

The corrosion rates of aluminum can be found in Table 4-21 through Table 4-22. Further explanation on the use of these rates can be found in Section 6.2.4. All aluminum alloy corrosion rates were converted to the same units of $\mu \mathrm{m} / \mathrm{yr}$ (Appendix II, aluminum_.xls). These converted rates are presented in this section along with their original values taken directly from the sources. 
Table 4-21. Corrosion Rates of Aluminum in Seawater

\begin{tabular}{|c|c|c|c|c|c|c|c|c|}
\hline \multirow[b]{2}{*}{ Type $^{\text {a }}$} & \multicolumn{4}{|c|}{ Weight Loss $(g)^{a}$} & \multicolumn{4}{|c|}{ Corrosion Rate $(\mu \mathrm{m} / \mathrm{yr})$} \\
\hline & 1 year & 2 years & 5 years & 10 years & 1 year & 2 years & 5 years & 10 years \\
\hline $1100-\mathrm{H} 14$ & 4.4 & 5.4 & 10.3 & 11.1 & 8.4 & 5.1 & 3.92 & 2.11 \\
\hline $3003-\mathrm{H} 14$ & 4.1 & 6.4 & 9.3 & 11.2 & 7.8 & 6.0 & 3.5 & 2.12 \\
\hline $5052-\mathrm{H} 34$ & 4.5 & 6.5 & 9.0 & 14.9 & 8.7 & 6.3 & 3.5 & 2.87 \\
\hline 6051-T4 & 3.7 & 4.9 & 9.9 & 12.3 & 7.1 & 4.7 & 3.8 & 2.35 \\
\hline 6051-T6 & 4.4 & 5.7 & 10.3 & 13.1 & 8.4 & 5.4 & 3.94 & 2.50 \\
\hline $6061-\mathrm{T} 4$ & 4.8 & 6.6 & 12.4 & 18.6 & 9.2 & 6.3 & 4.74 & 3.56 \\
\hline 6061-T6 & 5.5 & 7.7 & 14 & 21.5 & 11 & 7.4 & 5.4 & 4.11 \\
\hline 7072 & - & - & - & 10.2 & - & - & - & 1.87 \\
\hline 7075-T6 & - & - & - & 149 & - & - & - & 27.4 \\
\hline 5083 & 2.5 & 3.7 & - & 7.3 & 4.9 & 3.6 & - & 1.4 \\
\hline 5083 & 4.7 & 3.4 & 5.7 & 8.1 & 9.1 & 3.3 & 2.2 & 1.6 \\
\hline 5056 & 3.7 & 4.7 & 6.0 & 9.2 & 7.1 & 4.5 & 2.3 & 1.8 \\
\hline 5056 & 4.5 & 5.2 & - & 16.7 & 8.6 & 5.0 & - & 3.22 \\
\hline $6051-\mathrm{T} 4$ & 3.9 & 4.2 & 12.1 & 9.1 & 7.5 & 4.0 & 4.6 & 1.7 \\
\hline 6051-T6 & 4.1 & 4.5 & 7.7 & 10.6 & 7.8 & 4.3 & 2.9 & 2.03 \\
\hline 6053-T6 & 4.1 & 4.5 & 6.6 & 9.7 & 7.8 & 4.3 & 2.5 & 1.9 \\
\hline 6061-T6 & 7.6 & 13.4 & 29.4 & 51.6 & 15 & 13 & 11.2 & 9.86 \\
\hline 6061-T6 & 5.5 & 6.5 & 15.4 & 34.2 & 11 & 6.2 & 5.89 & 6.54 \\
\hline $\mathrm{Al}-7 \mathrm{Mg}$ & 4.1 & 4.1 & 6.5 & 9.4 & 7.8 & 3.9 & 2.5 & 1.8 \\
\hline 5154 & 2.8 & 5.2 & 6.0 & - & 5.4 & 5.0 & 2.3 & - \\
\hline 5083 & 3.5 & 4.6 & 6.0 & - & 6.8 & 4.5 & 2.3 & - \\
\hline 6053-T6 & 3.8 & 6.6 & 25.9 & - & 7.3 & 6.3 & 9.90 & - \\
\hline 7075-T6 & 60.4 & 49.3 & 74.8 & - & 111 & 45.3 & 27.5 & - \\
\hline 3003 , alclad & 4.3 & 12 & - & - & 8.1 & 11 & - & - \\
\hline 6061 , alclad & 4.3 & 3.9 & 5.7 & - & 8.2 & 3.7 & 2.2 & - \\
\hline 7075 , alclad & 4.4 & 5.2 & 6.1 & - & 8.1 & 4.8 & 2.2 & - \\
\hline $1100-\mathrm{H} 14$ & 1.9 & 3.5 & 5.3 & 12.7 & 3.6 & 3.3 & 2.0 & 2.42 \\
\hline $3003-\mathrm{H} 14$ & 0.0 & 3.3 & 4.6 & 7.5 & - & 3.1 & 1.7 & 1.4 \\
\hline $5052-\mathrm{H} 34$ & 2.8 & 3.3 & - & 14.2 & 5.4 & 3.2 & - & 2.73 \\
\hline
\end{tabular}


Table 4-21. Corrosion Rates of Aluminum in Seawater (Continued)

\begin{tabular}{|c|c|c|c|c|c|c|c|c|}
\hline \multirow[b]{2}{*}{ Type $^{a}$} & \multicolumn{4}{|c|}{ Weight Loss $(g)^{a}$} & \multicolumn{4}{|c|}{ Corrosion Rate $(\mu \mathrm{m} / \mathrm{yr})$} \\
\hline & 1 year & 2 years & 5 years & 10 years & 1 year & 2 years & 5 years & 10 years \\
\hline 6051-T4 & 0.0 & 0.7 & 3.5 & 8 & - & 0.7 & 1.3 & 2 \\
\hline 6051-T6 & 2.1 & 5.5 & 6.1 & 19.5 & 4.0 & 5.3 & 2.3 & 3.73 \\
\hline 6061-T4 & 4.4 & 6.0 & 8.0 & 15.6 & 8.4 & 5.7 & 3.1 & 2.98 \\
\hline 6061-T6 & 4.3 & 7.3 & 12.7 & 22.8 & 8.2 & 7.0 & 4.85 & 4.36 \\
\hline 7072 & - & - & - & 15.9 & - & - & - & 2.92 \\
\hline 7075-T6 & - & - & - & 242.6 & - & - & - & 44.55 \\
\hline 5083 & 2.8 & 0.0 & 6.1 & 8.5 & 5.4 & - & 2.4 & 1.6 \\
\hline 5083 & 2.6 & 3.2 & 5.2 & 7.5 & 5.0 & 3.1 & 2.0 & 1.5 \\
\hline 5056 & 2.5 & 3.3 & 5.7 & 10.4 & 4.8 & 3.2 & 2.2 & 2.00 \\
\hline 5056 & 4.0 & 4.1 & 5.5 & 11.1 & 7.7 & 3.9 & 2.1 & 2.14 \\
\hline 6051-T4 & 3.6 & 3.1 & 5.5 & 9.2 & 6.9 & 3.0 & 2.1 & 1.8 \\
\hline 6051-T6 & 5.3 & 4.1 & 8.4 & 18.6 & 10 & 3.9 & 3.2 & 3.56 \\
\hline 6053-T6 & 3.0 & 3.3 & 5.6 & 14.8 & 5.7 & 3.2 & 2.1 & 2.83 \\
\hline 6061-T6 & 9.8 & 11.2 & 33.2 & 48.5 & 19 & 10.7 & 12.7 & 9.27 \\
\hline 6061-T6 & 10.0 & 9.4 & 19.1 & 54.1 & 19.1 & 9.0 & 7.30 & 10.3 \\
\hline $\mathrm{Al}-7 \mathrm{Mg}$ & 2.4 & 2.4 & 4.6 & 8.0 & 4.6 & 2.3 & 1.8 & 1.5 \\
\hline 5154 & 2.4 & 2.6 & 3.8 & - & 4.7 & 2.5 & 1.5 & - \\
\hline 5083 & 2.0 & 2.8 & 3.6 & - & 3.9 & 2.7 & 1.4 & - \\
\hline 6053-T6 & 19.3 & 29.2 & 4.7 & - & 36.9 & 27.9 & 1.8 & - \\
\hline 7075-T6 & 44.8 & 66.1 & 116.0 & - & 82.3 & 60.70 & 42.60 & - \\
\hline 3003 , alclad & 1.6 & 2.3 & - & - & 3.0 & 2.2 & - & - \\
\hline 6061 , alclad & 8.4 & 3.3 & 6.5 & - & 16 & 3.2 & 2.5 & - \\
\hline 7075 , alclad & 2.8 & 3.6 & 6.8 & - & 5.1 & 3.3 & 2.5 & - \\
\hline $1100-\mathrm{H} 14$ & 0.0 & 2.4 & 1.3 & 2.3 & - & 2.3 & 0.50 & 0.44 \\
\hline $3003-\mathrm{H} 14$ & 0.0 & 0.0 & 3.0 & 2.2 & - & - & 1.1 & 0.42 \\
\hline $5052-\mathrm{H} 34$ & 1.7 & 0.0 & 0.0 & 0.6 & 3.3 & - & - & 0.1 \\
\hline 6051-T4 & 1.9 & 7.8 & 19.0 & 14.6 & 3.6 & 7.5 & 7.26 & 2.79 \\
\hline 6051-T6 & 22.5 & 13.8 & 19.9 & 27.3 & 43.0 & 13.2 & 7.61 & 5.22 \\
\hline 6061-T4 & 0.9 & 2.3 & 28.2 & 62 & 2 & 2.2 & 10.8 & 12 \\
\hline
\end{tabular}




\begin{tabular}{|c|c|c|c|c|c|c|c|c|}
\hline \multirow[b]{2}{*}{ Type $^{a}$} & \multicolumn{4}{|c|}{ Weight Loss $(\mathrm{g})^{\mathrm{a}}$} & \multicolumn{4}{|c|}{ Corrosion Rate $(\mu \mathrm{m} / \mathrm{yr})$} \\
\hline & 1 year & 2 years & 5 years & 10 years & 1 year & 2 years & 5 years & 10 years \\
\hline 6061-T6 & 6.7 & 7.1 & 11.1 & 44.3 & 13 & 6.8 & 4.24 & 8.47 \\
\hline 7072 & - & - & - & 3.1 & - & - & - & 0.59 \\
\hline 7075-T6 & - & - & - & 246.5 & - & - & - & 47.11 \\
\hline 5083 & 1.3 & 1.9 & 2.7 & 3.3 & 2.5 & 1.8 & 1.0 & 0.64 \\
\hline 5083 & 15.3 & 16.3 & 36.3 & 31.1 & 29.7 & 15.8 & 14.1 & 6.03 \\
\hline 5056 & 10.7 & 16.5 & 19.5 & 28.9 & 20.6 & 15.9 & 7.51 & 5.56 \\
\hline 5056 & 7.0 & 6.0 & 11.0 & 11.4 & 13 & 5.8 & 4.24 & 2.20 \\
\hline 6051-T4 & 9.1 & 18.8 & 15.3 & 51.0 & 17 & 18.0 & 5.85 & 9.75 \\
\hline 6051-T6 & 17.2 & 23.3 & 30.6 & 33.5 & 32.9 & 22.3 & 11.7 & 6.40 \\
\hline 6053-T6 & 15.7 & 25.1 & 19.3 & 25.8 & 30.0 & 24.0 & 7.38 & 4.93 \\
\hline 6061-T6 & 12.3 & 26.8 & 48.7 & 48.0 & 23.5 & 25.6 & 18.6 & 9.17 \\
\hline 6061-T6 & 7.3 & 7.0 & 21.3 & 18.6 & 14 & 6.7 & 8.14 & 3.56 \\
\hline $\mathrm{Al}-7 \mathrm{Mg}$ & 1.6 & 2.9 & 2.1 & 3.3 & 3.0 & 2.8 & 0.80 & 0.63 \\
\hline 5154 & 1.4 & 2.1 & 2.6 & - & 2.7 & 2.0 & 1.0 & - \\
\hline 5083 & 0.2 & 2.2 & 2.8 & - & 0.39 & 2.1 & 1.1 & - \\
\hline 6053-T6 & 45.6 & 80.4 & 86.0 & - & 87.1 & 76.8 & 32.9 & - \\
\hline 7075-T6 & 50.9 & 71.3 & 153.5 & - & 93.5 & 65.5 & 56.38 & - \\
\hline 3003, alclad & - & 1.9 & - & - & - & 1.8 & - & - \\
\hline 6061 , alclad & 20.8 & 15.8 & 34.3 & - & 39.8 & 15.1 & 13.1 & - \\
\hline 7075 , alclad & 8.5 & 14.5 & 16.6 & - & 16 & 13.3 & 6.10 & - \\
\hline
\end{tabular}

Source: ${ }^{\mathrm{a}}$ Hollingsworth and Hunsicker 1987 [DIRS 150403].

NOTE: Average temperature $\left(15.6^{\circ} \mathrm{C}\right)$ estimated from Figure 17 by Sedriks 1982 [DIRS 164923]. Temperature at Halifax, N.S., Esquimalt, B.C., and Kure Beach, NC are assumed similar. 
Table 4-22. Corrosion Rates of Aluminum in Freshwater

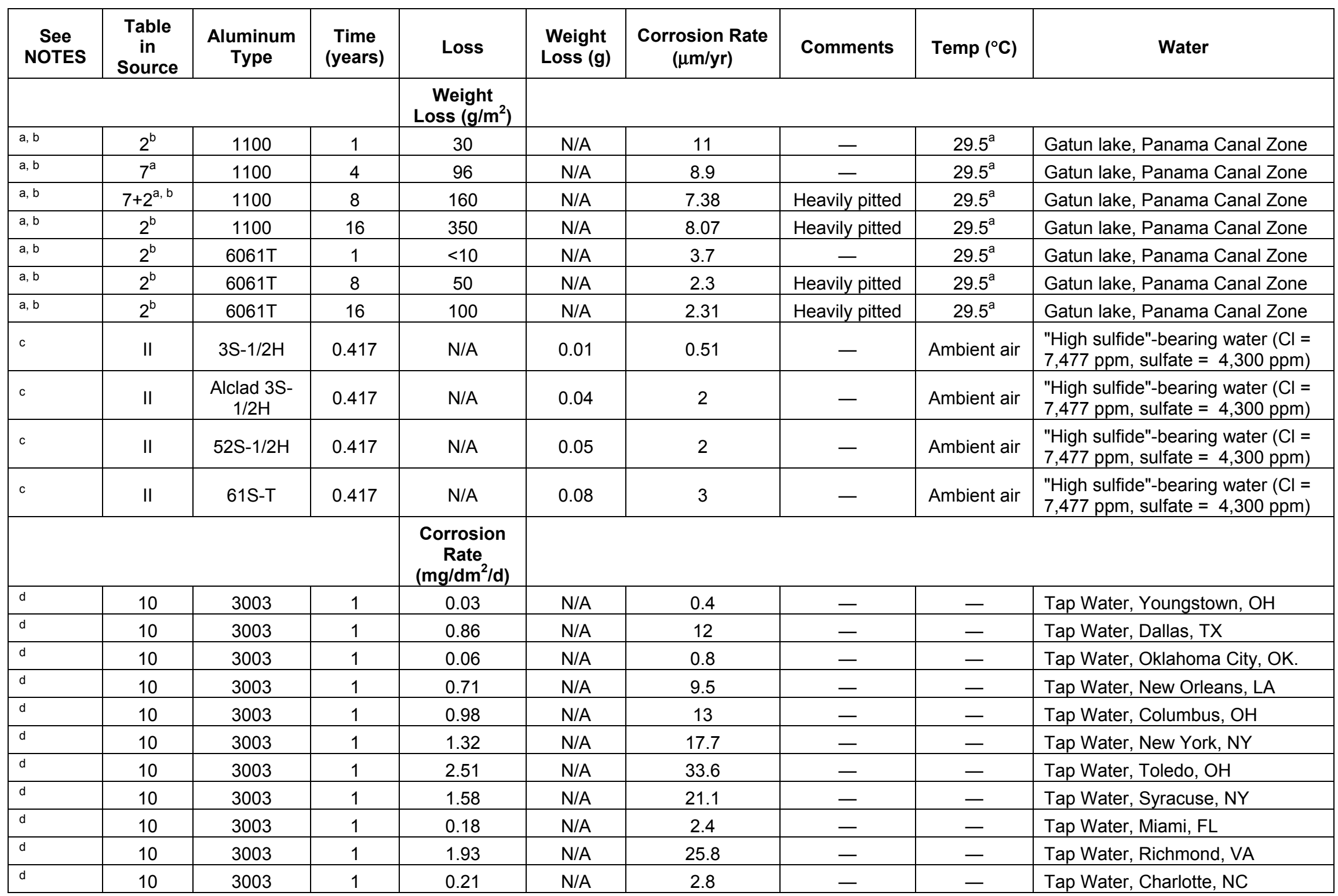




\begin{tabular}{|c|c|c|c|c|c|c|c|c|c|}
\hline $\begin{array}{c}\text { See } \\
\text { NOTES }\end{array}$ & $\begin{array}{c}\text { Table } \\
\text { in } \\
\text { Source }\end{array}$ & $\begin{array}{l}\text { Aluminum } \\
\text { Type }\end{array}$ & $\begin{array}{c}\text { Time } \\
\text { (years) }\end{array}$ & Loss & $\begin{array}{l}\text { Weight } \\
\text { Loss }(g)\end{array}$ & $\begin{array}{l}\text { Corrosion Rate } \\
(\mu \mathrm{m} / \mathrm{yr})\end{array}$ & Comments & Temp $\left({ }^{\circ} \mathrm{C}\right)$ & Water \\
\hline$d$ & 10 & 3003 & 1 & 1.01 & $\mathrm{~N} / \mathrm{A}$ & 13.5 & - & - & Tap Water, Little Rock, AR \\
\hline d & 10 & 3003 & 1 & 0.40 & N/A & 5.4 & - & - & Tap Water, Atlanta, GA \\
\hline - & 10 & 3003 & 1 & 0.33 & $\mathrm{~N} / \mathrm{A}$ & 4.4 & - & 一 & Tap Water, Portland, OR \\
\hline - & 10 & 3003 & 1 & 1.92 & N/A & 25.7 & 一 & 一 & Tap Water, Hartford, CT \\
\hline - & 10 & 3003 & 1 & 2.58 & $\mathrm{~N} / \mathrm{A}$ & 34.5 & - & - & Tap Water, Saginaw, MI \\
\hline - & 10 & 3003 & 1 & 0.04 & $\mathrm{~N} / \mathrm{A}$ & 0.5 & 一 & 一 & Tap Water, Minneapolis, MN \\
\hline - & 10 & 3003 & 1 & 1.77 & $\mathrm{~N} / \mathrm{A}$ & 23.7 & - & - & Tap Water, Boston, MA \\
\hline - & 10 & 3003 & 1 & 0.33 & N/A & 4.4 & - & 一 & Tap Water, Wichita, KS \\
\hline - & 10 & 3003 & 1 & 1.70 & $\mathrm{~N} / \mathrm{A}$ & 22.7 & - & 一 & Tap Water, Corpus Christi, TX \\
\hline - & 10 & 3003 & 1 & 0.38 & N/A & 5.1 & - & 一 & Tap Water, Birmingham, AL \\
\hline - & 10 & 3003 & 1 & 2.36 & $\mathrm{~N} / \mathrm{A}$ & 31.6 & - & - & Tap Water, Salt Lake City, UT \\
\hline - & 10 & 3003 & 1 & 1.95 & N/A & 26.1 & - & - & Tap Water, Milwaukee, WI \\
\hline - & 10 & 3003 & 1 & 2.76 & N/A & 36.9 & - & 一 & Tap Water, Philadelphia, PA \\
\hline - & 10 & 3003 & 1 & 1.52 & $\mathrm{~N} / \mathrm{A}$ & 20.3 & - & - & Tap Water, Nashville, TN \\
\hline - & 10 & 3003 & 1 & 1.78 & $\mathrm{~N} / \mathrm{A}$ & 23.8 & 一 & 一 & Tap Water, Los Angeles, CA \\
\hline - & 10 & 3003 & 1 & 0.47 & $\mathrm{~N} / \mathrm{A}$ & 6.3 & 一 & 一 & Tap Water, Chicago, IL \\
\hline - & 10 & 3003 & 1 & 0.23 & N/A & 3.1 & - & 一 & Tap Water, Pittsburgh, PA \\
\hline - & 10 & 3003 & 1 & 1.46 & $\mathrm{~N} / \mathrm{A}$ & 19.5 & - & 一 & Tap Water, Kansas City, MO \\
\hline - & 10 & 3003 & 1 & 1.21 & $\mathrm{~N} / \mathrm{A}$ & 16.2 & - & 一 & Tap Water, Washington, D.C. \\
\hline - & 10 & 3003 & 1 & 0.85 & $\mathrm{~N} / \mathrm{A}$ & 11 & - & 一 & Tap Water, Omaha, NE \\
\hline - & 10 & 3003 & 1 & 1.70 & N/A & 22.7 & - & 一 & Tap Water, Houston, TX \\
\hline 一 & 10 & 3003 & 1 & 2.06 & $\mathrm{~N} / \mathrm{A}$ & 27.6 & 一 & 一 & Tap Water, San Francisco, CA \\
\hline 一 & 10 & 3003 & 1 & 0.59 & N/A & 7.9 & - & - & Tap Water, Phoenix, AZ \\
\hline
\end{tabular}

Sources: a Forgeson et al. 1958 [DIRS 159343].

Southwell et al. 1976 [DIRS 100927].

Sawyer and Brown 1947 [DIRS 164921].

d Ailor 1969 [DIRS 164907], Table 10. 


\subsubsection{Zircaloy (2 and 4)}

Most information on the corrosion of zircaloy comes from high temperature experiments in which the weight gain over time is measured (i.e., Hillner et al. (1998 [DIRS 100455]) and Rothman 1984 [DIRS 100417]). Hillner et al. (1998 [DIRS 100455]) conclude that oxide growth in the Yucca Mountain repository over a period of one million years would be extremely small $(0.3$ mils, $1 \mathrm{mil}=25.4 \mu \mathrm{m})$.

\subsubsection{Alloy 22}

Since analysis of degradation rates for Alloy 22 can be found in other sources, no further analysis of this information will be provided in this analysis. This is discussed further in Section 6.2.8.

\subsubsection{Alloy N06464}

Corrosion data for a Ni-Cr-Mo-Gd alloy (UNS N06464, Table 6-2), is presented in Table 4-23.

Table 4-23. Corrosion Rates of Alloy N06464 in J-13 Water

\begin{tabular}{|c|c|c|}
\hline Time (years) & $\begin{array}{c}\text { Corrosion Rate } \\
\text { (nm/yr) }\end{array}$ & Fluid Type \\
\hline 0.03 & 415.8 & $\mathrm{~J}-13$ \\
\hline 0.08 & 55.7 & $\mathrm{~J}-13$ \\
\hline 0.20 & 62.1 & $\mathrm{~J}-13$ \\
\hline 0.27 & 48.5 & $\mathrm{~J}-13$ \\
\hline 0.39 & 77.4 & $\mathrm{~J}-13$ \\
\hline 0.77 & 20.1 & $\mathrm{~J}-13$ \\
\hline 0.62 & 88.9 & $\mathrm{~J}-1350 \times$ \\
\hline
\end{tabular}

Source: DOE 2004 [DIRS 168434] Table 16.

\subsubsection{Inconel Alloys}

The corrosion rates of Inconel alloys can be found in Table 4-24 through Table 4-26 below. Further explanation on the use of these rates can be found in Section 6.2.7. Inconel Alloy 625 is used in corrosion tests, which is assumed to have the same degradation rate as the Inconel alloys within the waste packages (Inconel Alloy Types 600 and X-750) (Section 5.2.1). 
Table 4-24. Corrosion Rates of Inconel Alloys in Freshwater

\begin{tabular}{|c|c|c|c|c|l|}
\hline $\begin{array}{c}\text { Time } \\
(\mathbf{y e a r s})\end{array}$ & $\begin{array}{c}\text { Corrosion Rate } \\
(\boldsymbol{\mu m} / \mathbf{y r})\end{array}$ & \multicolumn{1}{|c|}{ Table in Source } & $\begin{array}{c}\text { Temp } \\
\left({ }^{\circ} \mathbf{C}\right)\end{array}$ & $\begin{array}{c}\text { Type of } \\
\text { Analysis }\end{array}$ & \multicolumn{1}{|c|}{ Fluid Type } \\
\hline 0.51 & 0.05 & Sec.2.2.6, Sup.1, p.2.2-46 & 60 & WL & SDW J-13 Well Water \\
\hline 0.51 & 0.02 & Sec.2.2.6, Sup.1, p.2.2-47 & 60 & WL & SDW J-13 Well Water \\
\hline 0.51 & 0.01 & Sec.2.2.6, Sup.1, p.2.2-101 & 60 & WL & SDW J-13 Well Water \\
\hline 0.51 & 0.03 & Sec.2.2.6, Sup.2, p.2.2-102 & 60 & WL & SDW J-13 Well Water \\
\hline 0.51 & 0.04 & Sec.2.2.6, Sup.2, p.2.2-102 & 60 & WL & SDW J-13 Well Water \\
\hline 0.51 & 0.04 & Sec.2.2.6, Sup.2, p.2.2-103 & 60 & WL & SDW J-13 Well Water \\
\hline 0.51 & 0.01 & Sec.2.2.6, Sup.2, p.2.2-103 & 60 & WL & SDW J-13 Well Water \\
\hline 0.51 & 0.00 & Sec.2.2.6, Sup.2, p.2.2-103 & 60 & WL & SDW J-13 Well Water \\
\hline 0.50 & 0.10 & Sec.2.2.6, Sup.1, p.2.2-46 & 90 & WL & SDW J-13 Well Water \\
\hline 0.50 & 0.03 & Sec.2.2.6, Sup.1, p.2.2-47 & 90 & WL & SDW J-13 Well Water \\
\hline 0.50 & 0.01 & Sec.2.2.6, Sup.2, p.2.2-102 & 90 & WL & SDW J-13 Well Water \\
\hline 0.50 & 0.06 & Sec.2.2.6, Sup.2, p.2.2-102 & 90 & WL & SDW J-13 Well Water \\
\hline 0.50 & 0.03 & Sec.2.2.6, Sup.2, p.2.2-102 & 90 & WL & SDW J-13 Well Water \\
\hline 0.50 & 0.00 & Sec.2.2.6, Sup.2, p.2.2-103 & 90 & WL & SDW J-13 Well Water \\
\hline 0.50 & 0.02 & Sec.2.2.6, Sup.2, p.2.2-103 & 90 & WL & SDW J-13 Well Water \\
\hline 0.50 & 0.07 & Sec.2.2.6, Sup.2, p.2.2-103 & 90 & WL & SDW J-13 Well Water \\
\hline
\end{tabular}

Source: McCright 1998 [DIRS 114637].

NOTE: Negative corrosion values reported as zero in table. $\mathrm{WL}=$ weight loss; SDW = simulated dilute well.

Table 4-25. Corrosion Rates of Inconel Alloys in Acidified Water

\begin{tabular}{|c|c|c|c|c|c|}
\hline $\begin{array}{c}\text { Time } \\
\text { (years) }\end{array}$ & $\begin{array}{c}\text { Corrosion Rate } \\
(\mu \mathrm{m} / \mathrm{yr})\end{array}$ & Table in Source & $\begin{array}{c}\text { Temp } \\
\left({ }^{\circ} \mathrm{C}\right)\end{array}$ & $\begin{array}{c}\text { Type of } \\
\text { Analysis }\end{array}$ & Fluid Type \\
\hline 0.49 & 0.00 & Sec.2.2.6, Sup.1, p.2.2-45 & 60 & WL & SAW J-13 Well Water \\
\hline 0.49 & 0.00 & Sec.2.2.6, Sup.1, p.2.2-45 & 60 & WL & SAW J-13 Well Water \\
\hline 0.49 & 0.00 & Sec.2.2.6, Sup.1, p.2.2-45 & 60 & WL & SAW J-13 Well Water \\
\hline 0.96 & 0.00 & Sec.2.2.6, Sup.1, p.2.2-45 & 60 & WL & SAW J-13 Well Water \\
\hline 0.96 & 0.00 & Sec.2.2.6, Sup.1, p.2.2-45 & 60 & WL & SAW J-13 Well Water \\
\hline 0.96 & 0.00 & Sec.2.2.6, Sup.1, p.2.2-45 & 60 & WL & SAW J-13 Well Water \\
\hline 0.49 & 0.00 & Sec.2.2.6, Sup.1, p.2.2-46 & 60 & WL & SAW J-13 Well Water \\
\hline 0.49 & 0.03 & Sec.2.2.6, Sup.1, p.2.2-46 & 60 & WL & SAW J-13 Well Water \\
\hline 0.49 & 0.00 & Sec.2.2.6, Sup.1, p.2.2-46 & 60 & WL & SAW J-13 Well Water \\
\hline 0.96 & 0.00 & Sec.2.2.6, Sup.1, p.2.2-46 & 60 & WL & SAW J-13 Well Water \\
\hline 0.96 & 0.01 & Sec.2.2.6, Sup.1, p.2.2-46 & 60 & WL & SAW J-13 Well Water \\
\hline 0.96 & 0.00 & Sec.2.2.6, Sup.1, p.2.2-46 & 60 & WL & SAW J-13 Well Water \\
\hline 0.49 & 0.08 & Sec.2.2.6, Sup.2, p.2.2-101 & 60 & WL & SAW J-13 Well Water \\
\hline 0.49 & 0.03 & Sec.2.2.6, Sup.2, p.2.2-101 & 60 & WL & SAW J-13 Well Water \\
\hline 0.49 & 0.03 & Sec.2.2.6, Sup.2, p.2.2-101 & 60 & WL & SAW J-13 Well Water \\
\hline 0.96 & 0.03 & Sec.2.2.6, Sup.2, p.2.2-101 & 60 & WL & SAW J-13 Well Water \\
\hline 0.96 & 0.03 & Sec.2.2.6, Sup.2, p.2.2-101 & 60 & WL & SAW J-13 Well Water \\
\hline 0.96 & 0.02 & Sec.2.2.6, Sup.2, p.2.2-101 & 60 & WL & SAW J-13 Well Water \\
\hline 0.49 & 0.03 & Sec.2.2.6, Sup.2, p.2.2-102 & 60 & WL & SAW J-13 Well Water \\
\hline
\end{tabular}


Table 4-25. Corrosion Rates of Inconel Alloys in Acidified Water (Continued)

\begin{tabular}{|c|c|c|c|c|c|}
\hline $\begin{array}{c}\text { Time } \\
\text { (years) }\end{array}$ & $\begin{array}{c}\text { Corrosion Rate } \\
(\mu \mathrm{m} / \mathrm{yr})\end{array}$ & Table in Source & $\begin{array}{c}\text { Temp } \\
\left({ }^{\circ} \mathrm{C}\right)\end{array}$ & $\begin{array}{c}\text { Type of } \\
\text { Analysis } \\
\end{array}$ & Fluid Type \\
\hline 0.49 & 0.05 & Sec.2.2.6, Sup.2, p.2.2-102 & 60 & WL & SAW J-13 Well Water \\
\hline 0.49 & 0.08 & Sec.2.2.6, Sup.2, p.2.2-102 & 60 & WL & SAW J-13 Well Water \\
\hline 0.96 & 0.04 & Sec.2.2.6, Sup.2, p.2.2-102 & 60 & WL & SAW J-13 Well Water \\
\hline 0.96 & 0.06 & Sec.2.2.6, Sup.2, p.2.2-102 & 60 & WL & SAW J-13 Well Water \\
\hline 0.96 & 0.05 & Sec.2.2.6, Sup.2, p.2.2-102 & 60 & WL & SAW J-13 Well Water \\
\hline 0.50 & 0.00 & Sec.2.2.6, Sup.1, p.2.2-45 & 90 & WL & SAW J-13 Well Water \\
\hline 0.50 & 0.00 & Sec.2.2.6, Sup.1, p.2.2-45 & 90 & WL & SAW J-13 Well Water \\
\hline 0.50 & 0.00 & Sec.2.2.6, Sup.1, p.2.2-45 & 90 & WL & SAW J-13 Well Water \\
\hline 1.00 & 0.00 & Sec.2.2.6, Sup.1, p.2.2-45 & 90 & WL & SAW J-13 Well Water \\
\hline 1.00 & 0.00 & Sec.2.2.6, Sup.1, p.2.2-45 & 90 & WL & SAW J-13 Well Water \\
\hline 1.00 & 0.02 & Sec.2.2.6, Sup.1, p.2.2-45 & 90 & $W L$ & SAW J-13 Well Water \\
\hline 0.50 & 0.09 & Sec.2.2.6, Sup.1, p.2.2-46 & 90 & WL & SAW J-13 Well Water \\
\hline 0.50 & 0.00 & Sec.2.2.6, Sup.1, p.2.2-46 & 90 & WL & SAW J-13 Well Water \\
\hline 0.50 & 0.01 & Sec.2.2.6, Sup.1, p.2.2-46 & 90 & WL & SAW J-13 Well Water \\
\hline 1.00 & 0.00 & Sec.2.2.6, Sup.1, p.2.2-47 & 90 & WL & SAW J-13 Well Water \\
\hline 1.00 & 0.00 & Sec.2.2.6, Sup.1, p.2.2-47 & 90 & WL & SAW J-13 Well Water \\
\hline 1.00 & 0.00 & Sec.2.2.6, Sup.1, p.2.2-47 & 90 & WL & SAW J-13 Well Water \\
\hline 0.50 & 0.01 & Sec.2.2.6, Sup.2, p.2.2-101 & 90 & WL & SAW J-13 Well Water \\
\hline 0.50 & 0.02 & Sec.2.2.6, Sup.2, p.2.2-101 & 90 & WL & SAW J-13 Well Water \\
\hline 0.50 & 0.00 & Sec.2.2.6, Sup.2, p.2.2-101 & 90 & WL & SAW J-13 Well Water \\
\hline 1.00 & 0.00 & Sec.2.2.6, Sup.2, p.2.2-101 & 90 & WL & SAW J-13 Well Water \\
\hline 1.00 & 0.00 & Sec.2.2.6, Sup.2, p.2.2-101 & 90 & WL & SAW J-13 Well Water \\
\hline 1.00 & 0.00 & Sec.2.2.6, Sup.2, p.2.2-101 & 90 & WL & SAW J-13 Well Water \\
\hline 0.50 & 0.03 & Sec.2.2.6, Sup.2, p.2.2-102 & 90 & WL & SAW J-13 Well Water \\
\hline 0.50 & 0.00 & Sec.2.2.6, Sup.2, p.2.2-102 & 90 & WL & SAW J-13 Well Water \\
\hline 0.50 & 0.00 & Sec.2.2.6, Sup.2, p.2.2-102 & 90 & WL & SAW J-13 Well Water \\
\hline 1.00 & 0.00 & Sec.2.2.6, Sup.2, p.2.2-102 & 90 & WL & SAW J-13 Well Water \\
\hline 1.00 & 0.00 & Sec.2.2.6, Sup.2, p.2.2-102 & 90 & WL & SAW J-13 Well Water \\
\hline 1.00 & 0.00 & Sec.2.2.6, Sup.2, p.2.2-102 & 90 & WL & SAW J-13 Well Water \\
\hline
\end{tabular}

Source: McCright 1998 [DIRS 114637].

NOTE: Negative corrosion values reported as zero in table. $\mathrm{WL}=$ weight loss; SAW $=$ simulated acidified well. 
Table 4-26. Corrosion Rates of Inconel Alloys in Saltwater

\begin{tabular}{|c|c|c|c|c|c|}
\hline $\begin{array}{c}\text { Time } \\
\text { (years) }\end{array}$ & $\begin{array}{l}\text { Corrosion Rate } \\
(\mu \mathrm{m} / \mathrm{yr})\end{array}$ & Table in Source & $\begin{array}{l}\text { Temp } \\
\left({ }^{\circ} \mathrm{C}\right)\end{array}$ & $\begin{array}{l}\text { Type of } \\
\text { Analysis }\end{array}$ & Fluid Type \\
\hline 0.50 & 0.08 & Sec.2.2.6, Sup.1, p.2.2-46 & 60 & WL & SCW J-13 Well Water \\
\hline 0.50 & 0.05 & Sec.2.2.6, Sup.1, p.2.2-46 & 60 & WL & SCW J-13 Well Water \\
\hline 0.50 & 0.10 & Sec.2.2.6, Sup.1, p.2.2-46 & 60 & $\overline{W L}$ & SCW J-13 Well Water \\
\hline 0.50 & 0.10 & Sec.2.2.6, Sup.1, p.2.2-47 & 60 & WL & SCW J-13 Well Water \\
\hline 0.50 & 0.09 & Sec.2.2.6, Sup.1, p.2.2-47 & 60 & WL & SCW J-13 Well Water \\
\hline 0.50 & 0.06 & Sec.2.2.6, Sup.1, p.2.2-47 & 60 & $\overline{W L}$ & SCW J-13 Well Water \\
\hline 0.50 & 0.07 & Sec.2.2.6, Sup.2, p.2.2-101 & 60 & $\overline{W L}$ & SCW J-13 Well Water \\
\hline 0.50 & 0.05 & Sec.2.2.6, Sup.2, p.2.2-101 & 60 & WL & SCW J-13 Well Water \\
\hline 0.50 & 0.08 & Sec.2.2.6, Sup.2, p.2.2-101 & 60 & WL & SCW J-13 Well Water \\
\hline 0.50 & 0.08 & Sec.2.2.6, Sup.2, p.2.2-102 & 60 & $\overline{W L}$ & SCW J-13 Well Water \\
\hline 0.50 & 0.06 & Sec.2.2.6, Sup.2, p.2.2-102 & 60 & WL & SCW J-13 Well Water \\
\hline 0.50 & 0.10 & Sec.2.2.6, Sup.2, p.2.2-102 & 60 & $\overline{W L}$ & SCW J-13 Well Water \\
\hline 0.51 & 0.05 & Sec.2.2.6, Sup.1, p.2.2-46 & 90 & $\overline{W L}$ & SCW J-13 Well Water \\
\hline 0.51 & 0.07 & Sec.2.2.6, Sup.1, p.2.2-46 & 90 & WL & SCW J-13 Well Water \\
\hline 0.51 & 0.04 & Sec.2.2.6, Sup.1, p.2.2-46 & 90 & WL & SCW J-13 Well Water \\
\hline 0.51 & 0.04 & Sec.2.2.6, Sup.1, p.2.2-47 & 90 & WL & SCW J-13 Well Water \\
\hline 0.51 & 0.04 & Sec.2.2.6, Sup.1, p.2.2-47 & 90 & WL & SCW J-13 Well Water \\
\hline 0.51 & 0.03 & Sec.2.2.6, Sup.1, p.2.2-47 & 90 & WL & SCW J-13 Well Water \\
\hline 0.51 & 0.11 & Sec.2.2.6, Sup.2, p.2.2-101 & 90 & WL & SCW J-13 Well Water \\
\hline 0.51 & 0.10 & Sec.2.2.6, Sup.2, p.2.2-101 & 90 & WL & SCW J-13 Well Water \\
\hline 0.51 & 0.24 & Sec.2.2.6, Sup.2, p.2.2-101 & 90 & WL & SCW J-13 Well Water \\
\hline 0.51 & 0.06 & Sec.2.2.6, Sup.2, p.2.2-103 & 90 & WL & SCW J-13 Well Water \\
\hline 0.51 & 0.07 & Sec.2.2.6, Sup.2, p.2.2-103 & 90 & WL & SCW J-13 Well Water \\
\hline 0.51 & 0.12 & Sec.2.2.6, Sup.2, p.2.2-103 & 90 & WL & SCW J-13 Well Water \\
\hline
\end{tabular}

Source: McCright 1998 [DIRS 114637].

NOTE: Negative corrosion values reported as zero in table.

$\mathrm{WL}=$ weight loss; SCW = simulated concentrated well.

\subsubsection{Chrome Plating}

Due to the extremely limited use of chrome plating in waste packages (chrome plating of borated Stainless Steel Type 304L poison wafers in Shippingport PWR assemblies-Section 4.1.1.2), the corrosion of chrome plating will not be investigated in this analysis.

\subsubsection{Densities of Steels and Alloys}

In several cases, conversion of rates from referenced sources to $\mu \mathrm{m} / \mathrm{yr}$ was necessary to obtain a consistent unit of measure of corrosion for this analysis. This was required when the corrosion rate was given as a mass loss per time. To complete the conversions (Appendix II), the densities of the materials (Table 4-27) had to be used. 
Table 4-27. Material Densities

\begin{tabular}{|c|c|c|c|}
\hline \multicolumn{4}{|c|}{ Aluminum Alloys } \\
\hline Type & Density $\left(\mathrm{g} / \mathrm{cm}^{3}\right)$ & Type & Density $\left(\mathrm{g} / \mathrm{cm}^{3}\right)$ \\
\hline 1100 & 2.71 & 5154 & 2.66 \\
\hline 3003 & 2.73 & 6061 & 2.70 \\
\hline 5052 & 2.68 & 7075 & 2.81 \\
\hline 5083 & 2.66 & & \\
\hline \multicolumn{4}{|c|}{ Stainless Steels } \\
\hline Type & Density $\left(\mathrm{g} / \mathrm{cm}^{3}\right)$ & Type & Density $\left(\mathrm{g} / \mathrm{cm}^{3}\right)$ \\
\hline 302 & 7.94 & 316 & 7.98 \\
\hline 304 & 7.94 & $316 \mathrm{~L}$ & 7.98 \\
\hline $304 \mathrm{~L}$ & 7.94 & 321 & 7.94 \\
\hline
\end{tabular}

Source: ASTM G 1-90 [DIRS 103515].

\subsubsection{Miscellaneous Materials}

Miscellaneous materials, as defined in this analysis, are waste package component materials that do not fit neatly into one of the three categories specified above (i.e., fuel types or waste forms, steels, or alloys). These materials are presented below.

\subsubsection{Sintered Compounds}

The sintered compounds here are those contained within the Fort Saint Vrain fuel elements. The fuel elements are graphite blocks that hold the fuel compacts, which contain the fuel kernels. Fuel kernels are composed of a thorium carbide or $\mathrm{Th} / \mathrm{U}$ carbide core surrounded by four protective coatings of $\mathrm{SiC}$ and pyrolytic carbon. These kernels are mixed with a carbonaceous binder material and "baked" to create the fuel compacts (Taylor 2001 [DIRS 154726]). This section is concerned with fuel element materials that do not contain fissile material (graphite, pyrolytic carbon, and $\mathrm{SiC}$ ).

\subsection{Graphite and Pyrolytic Carbon}

The oxidation data for graphite and pyrolytic carbon are found in Table 4-28. 
Table 4-28. Corrosion Behavior of Sintered Carbon Materials

\begin{tabular}{|c|c|}
\hline Reference & Corrosion/Oxidation Rate/Behavior \\
\hline Lide 2002 [DIRS 160832], p. 4-50 & Insoluble in $\mathrm{H}_{2} \mathrm{O}$ \\
\hline Lewis 1990 [DIRS 130543], p. 188 & For Industrial graphite: Excellent corrosion resistance \\
\hline $\begin{array}{l}\text { Lotts et al. } 1992 \text { [DIRS 164916], } \\
\text { Appendix C }\end{array}$ & $\begin{array}{l}\text { Report air oxidation of graphite: at } 150^{\circ} \mathrm{C}=1.05 \times 10^{-17} \mathrm{~g} / \mathrm{cm}^{2} / \mathrm{s} \text {; at } \\
100^{\circ} \mathrm{C}=4.5 \times 10^{-20} \mathrm{~g} / \mathrm{cm}^{2} / \mathrm{s} \text {. Corrosion by water is known to be slower } \\
\text { than that in air (Propp } 1998 \text { [DIRS } 149395]) . \text { Therefore, at } 25^{\circ} \mathrm{C} \text { in } \mathrm{H}_{2} \mathrm{O} \text {, } \\
\text { the graphite can be assumed insoluble. }\end{array}$ \\
\hline Morgan 1981 [DIRS 164920], p. 18 & Insoluble at "ordinary temperatures" \\
\hline Gurwell 1981 [DIRS 164911] & $\begin{array}{l}\text { Reacts very slowly in deionized water }\left(250^{\circ} \mathrm{C}=10^{-7} \mathrm{~g} / \mathrm{cm}^{2} / \text { day, }\right. \\
\left.\text { extrapolated to } 99^{\circ} \mathrm{C}=10^{-10} \mathrm{~g} / \mathrm{cm}^{2} / \text { day }\right)\end{array}$ \\
\hline $\begin{array}{l}\text { Shreir et al. } 1998 \text { [DIRS 164925], } \\
\text { Chapter } 18\end{array}$ & $\begin{array}{l}\text { Generally unreactive at low temperatures. Table } 18.2 \text { shows that it is } \\
\text { completely resistant to a great number of highly corrosive environments. }\end{array}$ \\
\hline Propp 1998 [DIRS 149395], p. 21 & $\begin{array}{l}\text { Air oxidation of graphite at } 400^{\circ} \mathrm{C}=1 \mathrm{mg} / \mathrm{cm}^{2} \text { in } 1.9 \text { years and at } \\
200^{\circ} \mathrm{C}=1 \mathrm{mg} / \mathrm{cm}^{2} \text { in } 190,000 \text { years }\end{array}$ \\
\hline
\end{tabular}

\subsection{Silicon Carbide (SiC)}

Most information on the oxidation of $\mathrm{SiC}$ comes from high temperature data in humid atmospheres, many times with the temperatures exceeding $1,000^{\circ} \mathrm{C}$. Those data are located in Table 4-29. In Section 6.3.1, these high temperature data are used to approximate an oxidation rate of $\mathrm{SiC}$ at $25^{\circ} \mathrm{C}$. Lide (2002 [DIRS 160832]) indicates that $\mathrm{SiC}$ is insoluble in water at $25^{\circ} \mathrm{C}$.

Table 4-29. High Temperature Values for SiC Oxidation

\begin{tabular}{|c|c|c|}
\hline Temperature $\left({ }^{\circ} \mathrm{C}\right)$ & Condition & Rate $\left(\mathrm{mg} / \mathrm{cm}^{2} \cdot \mathrm{h}\right)$ \\
\hline 1,100 & $10 \%$ vol $\mathrm{H}_{2} \mathrm{O} / \mathrm{O}_{2}$ & $2.96 \times 10^{-3}$ \\
\hline 1,100 & $25 \%$ vol $\mathrm{H}_{2} \mathrm{O} / \mathrm{O}_{2}$ & $6.52 \times 10^{-3}$ \\
\hline 1,100 & $50 \%$ vol H${ }_{2} \mathrm{O} / \mathrm{O}_{2}$ & $4.34 \times 10^{-3}$ \\
\hline 1,100 & $70 \%$ vol $\mathrm{H}_{2} \mathrm{O} / \mathrm{O} 2$ & $3.32 \times 10^{-3}$ \\
\hline 1,100 & $90 \%$ vol $\mathrm{H}_{2} \mathrm{O} / \mathrm{O}_{2}$ & $2.83 \times 10^{-3}$ \\
\hline 1,100 & 14 vol\% $\mathrm{H}_{2} \mathrm{O} /$ Argon & $1.85 \times 10^{-3}$ \\
\hline 1,100 & 32 vol\% $\mathrm{H}_{2} \mathrm{O} /$ Argon & $3.65 \times 10^{-3}$ \\
\hline 1,100 & 59 vol\% $\mathrm{H}_{2} \mathrm{O} /$ Argon & $4.45 \times 10^{-3}$ \\
\hline 1,100 & 93 vol\% $\mathrm{H}_{2} \mathrm{O} /$ Argon & $4.46 \times 10^{-3}$ \\
\hline 1,200 & $10 \%$ vol $\mathrm{H}_{2} \mathrm{O} / \mathrm{O}_{2}$ & $3.05 \times 10^{-2}$ \\
\hline 1,200 & $50 \%$ vol $\mathrm{H}_{2} \mathrm{O} / \mathrm{O}_{2}$ & $1.27 \times 10^{-2}$ \\
\hline 1,200 & $70 \%$ vol $\mathrm{H}_{2} \mathrm{O} / \mathrm{O}_{2}$ & $2.11 \times 10^{-2}$ \\
\hline 1,200 & $90 \%$ vol $\mathrm{H}_{2} \mathrm{O} / \mathrm{O}_{2}$ & $9.06 \times 10^{-2}$ \\
\hline
\end{tabular}

Source: Temperature data from Opila 1999 [DIRS 155502], Table II.

\subsubsection{Gadolinium Phosphate $\left(\mathrm{GdPO}_{4}\right)$}

In this analysis, the mineral monazite, a mixed lanthanide orthophosphate, is assumed to be a natural analog for $\mathrm{GdPO}_{4}$ (Section 5.3.1). Natural mineral deposits of monazite have been reported to be over 2 billion years old and alluvial deposits indicate that the mineral has survived multiple sedimentation and metamorphic cycles occurring over several hundred million years 
(Boatner and Sales 1988 [DIRS 164908]). This indicates that the mineral (in its natural form) is extremely resistant to corrosion.

Tests on synthetic monazite indicate that $<0.001 \mathrm{~g} / \mathrm{m}^{2}$.day of the lanthanide was released from the material and that the monazite matrix had a corrosion rate of less than $0.002 \mathrm{~g} / \mathrm{m}^{2} \cdot \mathrm{d}$ in water at $90^{\circ} \mathrm{C}$ (Boatner and Sales 1988 [DIRS 164908], Figure 23 and Section 8.2). However, this was not a pure form of the substance, but was combined with $20 \mathrm{wt} \%$ Savannah River defense waste. Therefore, corrosion of pure monazite is expected to be much lower, since mineral impurities usually cause crystal structure defects or stress, which leads to increased mineral solubility.

\subsubsection{Miscellaneous Handbook Data}

Table 4-30 contains various constants used in the spreadsheet analyses for conversions and calculating values of corrosion rates.

Table 4-30. Miscellaneous Constants Used in Conversions

\begin{tabular}{|c|c|c|c|c|c|}
\hline \multicolumn{6}{|c|}{ Constants (Lide 2002 [DIRS 160832]) } \\
\hline \multicolumn{2}{|c|}{ Term } & \multicolumn{2}{|c|}{ Value } & \multicolumn{2}{|c|}{ Source } \\
\hline \multicolumn{2}{|l|}{ Gas Constant (R) } & \multicolumn{2}{|c|}{$8.314510 \mathrm{~Pa} \mathrm{~m}^{3} \mathrm{~K}^{-1} \mathrm{~mol}^{-1}$} & \multicolumn{2}{|c|}{ p. $1-54$} \\
\hline \multicolumn{2}{|l|}{ Kelvin (K) } & \multicolumn{2}{|c|}{${ }^{\circ} \mathrm{C}+273.15$} & \multicolumn{2}{|c|}{ p. 1-46 } \\
\hline \multicolumn{2}{|l|}{ Celsius $\left({ }^{\circ} \mathrm{C}\right)$} & \multicolumn{2}{|c|}{$(5 / 9) \times\left({ }^{\circ} \mathrm{F}-32\right)$} & \multicolumn{2}{|c|}{ p. $1-46$} \\
\hline \multicolumn{2}{|l|}{ SiC density } & \multicolumn{2}{|c|}{$3.16 \mathrm{~g} / \mathrm{cm}^{3}$} & \multicolumn{2}{|c|}{ p. 4-82 } \\
\hline \multicolumn{2}{|l|}{ Graphite density } & \multicolumn{2}{|c|}{$2.2 \mathrm{~g} / \mathrm{cm}^{3}$} & \multicolumn{2}{|c|}{ p. $4-50$} \\
\hline \multicolumn{6}{|c|}{ Atomic weight of elements (Parrington et al. 1996 [DIRS 103896]) } \\
\hline Element & $\begin{array}{l}\text { ATWT } \\
\text { (grams/mole) }\end{array}$ & Element & $\begin{array}{l}\text { ATWT } \\
\text { (grams/mole) }\end{array}$ & Element & $\begin{array}{l}\text { ATWT } \\
\text { (grams/mole) }\end{array}$ \\
\hline $\mathrm{Gd}$ & 157.25 & $\mathrm{P}$ & 30.973761 & $\mathrm{O}$ & 15.9994 \\
\hline $\mathrm{Cl}$ & 35.4527 & $\mathrm{~F}$ & 18.9984032 & & \\
\hline
\end{tabular}

\subsubsection{Titanium Grades 7 and 24}

\subsubsection{Titanium Grade 7}

Since analysis of corrosion rates for Titanium Grade 7 can be found in other sources, this data will not by analyzed in this document. Information about other sources for this data is provided in Section 6.5.1.

\subsubsection{Titanium Grade 24}

Titanium Grade 24, an alloy with $\sim 6 \mathrm{wt} \% \mathrm{Al}$ and $4 \mathrm{wt} \% \mathrm{~V}$ with and $\sim 0.04$ to $0.08 \mathrm{wt} \% \mathrm{Pd}$, is used as the structural material in the design of the drip shield. An experimentally obtained corrosion rate for Titanium Grade 24 under repository conditions is not available at this time. However, the comparative corrosion behavior of Titanium Grade 24 can be estimated based on available data for Titanium Grade 7 (See Section 6.5.2).

The LTCTF (Long Term Corrosion Test Facility) has obtained corrosion rate data for Titanium Grade 7 exposed in both vapor and liquid phases of SAW, SCW and SDW at $60^{\circ} \mathrm{C}$ and $90^{\circ} \mathrm{C}$ for 
two and a half (2.5) years with weight-loss specimens (DTN: LL030410012251.056 [DIRS 169583]). The 2.5-year Titanium Grade 7 aqueous corrosion rate data are shown in Table 4-31 and are used to calculate the corrosion rate for Titanium Grade 24 (Section 6.5.2).

Table 4-31. Titanium Grade 7 Weight-Loss Corrosion Rates in Aqueous Media (2.5-Year Data)

\begin{tabular}{|c|c|c|c|}
\hline $\begin{array}{c}\text { Corrosion Rate } \\
\text { (nm/yr) }\end{array}$ & $\begin{array}{l}\text { Temp } \\
\left({ }^{\circ} \mathrm{C}\right)\end{array}$ & $\begin{array}{l}\text { Type of } \\
\text { Analysis }\end{array}$ & Fluid Type \\
\hline 0.00 & 60 & WL & Aqueous Phase SAW \\
\hline 0.00 & 60 & WL & Aqueous Phase SAW \\
\hline 0.00 & 60 & WL & Aqueous Phase SAW \\
\hline 0.00 & 90 & WL & Aqueous Phase SAW \\
\hline 2.91 & 90 & WL & Aqueous Phase SAW \\
\hline 14.59 & 90 & WL & Aqueous Phase SAW \\
\hline 23.37 & 90 & WL & Aqueous Phase SCW \\
\hline 2.92 & 90 & WL & Aqueous Phase SCW \\
\hline 26.30 & 90 & WL & Aqueous Phase SCW \\
\hline 43.57 & 90 & WL & Aqueous Phase SCW \\
\hline 43.63 & 90 & WL & Aqueous Phase SCW \\
\hline 46.29 & 90 & WL & Aqueous Phase SCW \\
\hline 2.89 & 60 & WL & Aqueous Phase SDW \\
\hline 5.77 & 60 & WL & Aqueous Phase SDW \\
\hline 2.89 & 60 & WL & Aqueous Phase SDW \\
\hline 0.00 & 90 & WL & Aqueous Phase SDW \\
\hline 0.00 & 90 & WL & Aqueous Phase SDW \\
\hline 0.00 & 90 & WL & Aqueous Phase SDW \\
\hline 0.00 & 60 & WL & Aqueous Phase SAW \\
\hline 0.00 & 60 & WL & Aqueous Phase SAW \\
\hline 2.95 & 60 & WL & Aqueous Phase SAW \\
\hline 17.64 & 90 & WL & Aqueous Phase SAW \\
\hline 2.94 & 90 & WL & Aqueous Phase SAW \\
\hline 2.94 & 90 & WL & Aqueous Phase SAW \\
\hline 11.83 & 90 & WL & Aqueous Phase SCW \\
\hline 14.83 & 90 & WL & Aqueous Phase SCW \\
\hline 17.64 & 90 & WL & Aqueous Phase SCW \\
\hline 43.75 & 90 & WL & Aqueous Phase SCW \\
\hline 49.70 & 90 & WL & Aqueous Phase SCW \\
\hline 49.46 & 90 & WL & Aqueous Phase SCW \\
\hline 0.00 & 60 & WL & Aqueous Phase SDW \\
\hline 0.00 & 60 & WL & Aqueous Phase SDW \\
\hline 0.00 & 60 & WL & Aqueous Phase SDW \\
\hline 0.00 & 90 & WL & Aqueous Phase SDW \\
\hline 0.00 & 90 & WL & Aqueous Phase SDW \\
\hline 2.92 & 90 & WL & Aqueous Phase SDW \\
\hline
\end{tabular}




\subsection{CRITERIA}

The technical work plan for this activity (BSC 2004 [DIRS 171583], Table 3-1) has identified the following acceptance criteria (AC) based on the requirements mentioned in Project Requirements Document (PRD) (Canori and Leitner 2003 [DIRS 166275]) and Yucca Mountain Review Plan, Final Report (NRC 2003 [DIRS 163274]):

System Description and Demonstration of Multiple Barriers (NRC 2003 [DIRS 163274], Section 2.2.1.1.3; Canori and Leitner 2003 [DIRS 166275], PRD-002/T-014, PRD-002/T-016)

Specific requirements involve identifying multiple barriers (natural and engineered), describing the capabilities of these barriers to isolate waste, and providing technical bases for capabilities descriptions consistent with the postclosure performance objectives. To comply with these requirements, the following acceptance criteria are identified in the technical work plan (BSC 2004 [DIRS 171583], Table 3-1):

- AC1: Identification of Barriers is Adequate

- AC2: Description of Barrier Capability to Isolate Waste is Acceptable

- AC3: Technical Basis for Barrier Capability is Adequately Presented.

Degradation of Engineered Barriers (NRC 2003 [DIRS 163274], Section 2.2.1.3.1.3; Canori and Leitner 2003 [DIRS 166275], PRD-002/T-015)

Specific requirements include describing deterioration or degradation of engineered barriers and modeling degradation processes using data for performance assessment, including total system performance assessment (TSPA). Consideration of uncertainties and variabilities in model parameters and alternative conceptual models are also required. To fulfill these requirements, the following acceptance criteria are identified in the technical work plan (BSC 2004 [DIRS 171583], Table 3-1):

- AC1: System Description and Model Integration are Adequate

- AC2: Data are Sufficient for Model Justification

- AC3: Data Uncertainty is Characterized and Propagated Through the Model Abstraction

- AC4: Model Uncertainty is Characterized and Propagated Through the Model Abstraction

- AC5: Model Abstraction Output is Supported by Objective Comparisons.

\subsection{CODES, STANDARDS, AND REGULATIONS}

The following standards and regulations were used in Sections 4 and 6 and Appendix II of this analysis:

- ASTM A 240/A 240M-03b. 2003. Standard Specification for Chromium and Chromium-Nickel Stainless Steel Plate, Sheet, and Strip for Pressure Vessels and for General Applications 
- ASTM A 276-03. 2003. Standard Specification for Stainless Steel Bars and Shapes

- ASTM A 516/A 516M-01. 2001. Standard Specification for Pressure Vessel Plates, Carbon Steel, for Moderate- and Lower-Temperature Service

- ASTM A 887-89 (Reapproved 2000). 2000. Standard Specification for Borated Stainless Steel Plate, Sheet, and Strip for Nuclear Application

- ASTM B 209-96. 1996. Standard Specification for Aluminum and Aluminum-Alloy Sheet and Plate

- ASTM B 265-02. 2002. Standard Specification for Titanium and Titanium Alloy Strip, Sheet, and Plate

- ASTM B 932-04. 2004. Standard Specification for Low-Carbon Nickel-ChromiumMolybdenum-Gadolinium Alloy Plate, Sheet, and Strip

- ASTM G 1-90 (Reapproved 1999). 1999. Standard Practice for Preparing, Cleaning, and Evaluating Corrosion Test Specimens

- 10 CFR 63. Energy: Disposal of High-Level Radioactive Wastes in a Geologic Repository at Yucca Mountain, Nevada

- 48 FR 28194. 10 CFR Part 60 Disposal of High-Level Radioactive Wastes in Geologic Repositories Technical Criteria. 


\section{ASSUMPTIONS}

\subsection{ASSUMPTIONS CONCERNING STEEL TYPES INVESTIGATED IN THIS ANALYSIS}

\subsubsection{AM-350 Corrosion Rate}

Assumption: Published degradation rates were not found for Stainless Steel Type AM-350. For this analysis, the degradation rate is assumed to be the same as the degradation rate of Stainless Steel Type 316/316L.

Rationale: These stainless steels are very similar compositionally (Table 6-2) and are, therefore, likely to have similar degradation rates.

Confirmation Status: Information by Hudson and Stanners (1955 [DIRS 159334]), Scarberry et al. (1967 [DIRS 159335]), and Wallen and Olsson (1977 [DIRS 164948]) indicate the strong dependence of corrosion on metal composition, especially alloying elements. This indicates that compositionally similar metals will corrode at similar rates. Therefore, no further confirmation is required.

Use in the Analysis: This assumption is used in Section 6.2.1.2 in which the rates for Stainless Steel Types 316, 316L, and AM-350 are considered to have the same corrosion rates as presented in Table 6-5 and Figure 6-3 through Figure 6-5.

\subsubsection{Use of Corrosion of Stainless Steel Type 302}

Assumption: Published degradation rates for Stainless Steel Type 302 can be used for the corrosion of Stainless Steel Type 304 and 304L.

Rationale: The basis for this assumption is that these stainless steels are very similar compositionally (Table 6-2) and are, therefore, likely to have similar degradation rates.

Confirmation Status: Information in Hudson and Stanners (1955 [DIRS 159334]), Scarberry et al. (1967 [DIRS 159335]), and Wallen and Olsson (1977 [DIRS 164948]) indicate the strong dependence of corrosion to metal composition, especially alloying elements. This indicates that compositionally similar metals will corrode at similar rates. Therefore, no further confirmation is required.

Use in the Analysis: This assumption is used in Appendix II (ECDF_metals.xls) in which the corrosion rates for Stainless Steel Types 304, 304L, and 302 were combined to create the statistical information for these three steel types for use in Section 6.2.1.

\subsection{ASSUMPTIONS CONCERNING ALLOYS INVESTIGATED IN THIS ANALYSIS}

\subsubsection{Corrosion of Inconel Alloys 600 and $X-750$}

Assumption: Published degradation rates were not found for Inconel Alloys 600 and X-750. For this analysis, the degradation rate was assumed the same as the Inconel Alloy 625 degradation rate. 
Rationale: The basis for this assumption is that these alloys are similar compositionally (Table 6-2) and are, therefore, likely to have similar degradation rates.

Confirmation Status: Information provided by Hudson and Stanners (1955 [DIRS 159334]), Scarberry et al. (1967 [DIRS 159335]), and Wallen and Olsson (1977 [DIRS 164948]) indicates the strong dependence of corrosion on metal composition, especially alloying elements. This indicates that compositionally similar metals will corrode at similar rates. Therefore, no further confirmation is required.

Use in the Analysis: This assumption is used in Appendix II (aqueous Inconel-Alloy_.xls) in which these alloys are referred to only as Inconel alloy. These rates were then used for all the statistical information presented in Section 6.2.6.

\subsubsection{Density of Aluminum Alloys}

Assumption: Published density values were not found for several aluminum alloys. The density for Aluminum Alloy Type 5056 is assumed the same as Aluminum Alloy Type 5052; Aluminum Alloy Types 6051 and 6053 (all tempers) are assumed the same as Aluminum Alloy Type 6061; and Aluminum Alloy Type 7072 was assumed the same as Aluminum Alloy Type 7075 . For alloys for which there is a core material surrounded by a different "clad" alloy, the density of the core was taken as the density of the sample.

Rationale: For single metal specimens, the basis for this assumption is that the alloys are similar compositionally (Table 6-2), and are, therefore, likely to have similar densities. For clad material, since the metal core comprises the majority of the volume of the specimen, it is logical that the density of the specimen would be similar to that of the core material.

Confirmation Status: As shown in Table 6-2, aluminum alloys range in density from 2.81 to $2.68 \mathrm{~g} / \mathrm{cm}^{3}$. In addition, it can be seen from this table that compositionally similar metals have the same density (i.e., Aluminum Alloy Types 5052 and 5056). Because of this similarity, no further confirmation is required.

Use in the Analysis: This assumption is used in Appendix II (aluminum_.xls) to calculate the corrosion rate from $\mathrm{g}$ to $\mu \mathrm{m} / \mathrm{yr}$ for use in the statistical analysis presented in ECDF_metals.xls and Section 6.2.4.

\subsection{ASSUMPTIONS CONCERNING MISCELLANEOUS MATERIALS}

\subsection{1 $\quad \mathrm{GdPO}_{4}$ Mineralogy}

Assumption: The general properties and density of $\mathrm{GdPO}_{4}$ were taken to be the same as those of the mineral monazite ((Ce,La,Nd,Th) $\left.\mathrm{PO}_{4}\right)$.

Rationale: The rationale is that since gadolinium is a lanthanide and will have similar properties to the actinide and lanthanides occurring in monazite, monazite properties can be used to bound characteristics of $\mathrm{GdPO}_{4}$.

Confirmation Status: This assumption is considered realistic. Therefore, no further confirmation is required. 
Use in the Analysis: This assumption is used in Appendix II (Misc_Materials.xls) to calculate a rate in units of $\mu \mathrm{m} / \mathrm{yr}$ for use in Section 6.3.3. It is also used in Section 6.3.3 for verification of the durability of $\mathrm{GdPO}_{4}$.

\subsection{ASSUMPTIONS CONCERNING GLASS FRACTURING}

\subsubsection{Cooling Fractures}

Assumption: The fracture factor corresponding to glass cooling is assumed to be 12.5 .

Rationale: The rationale is that a fracture factor range for typical cooling rates of DHLW glass is 10 to 15 (CRWMS M\&O 1998 [DIRS 100362], p. 6-79). The average of this is 12.5, which also corresponds to a fracture factor for free air convection cooled glass (CRWMS M\&O 1998 [DIRS 100362], Figure 6-32).

Confirmation status: This assumption is considered to be realistic. Therefore, no further confirmation is required.

Use in the analysis: This assumption is used in Appendix II (glass-fracturefac.xls) to calculate the fracture factor of high-level waste glass as presented in Table 6-16 and Table 6-17 in Section 6.4.3.1.

\subsubsection{Fractures Due to a Drop}

Assumption: The fracture factor corresponding to fractures created in a situation where the glass pour canisters are dropped is 5.

Rationale: Smith and Ross (1975 [DIRS 102088], pp. 12 to 14 and Figure 41) indicate that if a drop occurred, facilities under consideration would have a usual drop distance of 10 feet. A 30-foot drop would be near the upper limit of the drop distance and also matches the transportation packing requirements. Therefore, the upper limit of 30 feet was used for this analysis to derive the fractional increase in surface area for dropped canisters. Figure 41 of Impact Testing of Vitreous Simulated High-Level Waste in Canisters (Smith and Ross 1975 [DIRS 102088]) shows that at the velocity the package would be traveling when impact occurred from 30 feet (44 feet per second, (Smith and Ross 1975 [DIRS 102088], Table 1) the corresponding fractional increase in surface area (from best estimate curve) would be approximately 4. A value of 5 (4 plus 1 for the geometric surface area) is therefore used here.

Confirmation status: This assumption is considered to be realistic. Therefore, no further confirmation is required.

Use in the analysis: This assumption is used in Appendix II (glass-fracturefac.xls) to calculate the fracture factor of high-level waste glass as presented in Table 6-16 and Table 6-17 in Section 6.4.3.1. 


\subsubsection{Availability of Cooling Fractures}

Assumption: Fractures created during glass cooling will not add to the degradation rate of the high level waste glass in a glass pour canister that has not been dropped.

Rationale: Most references dealing with fractures discuss how surface area increases. To obtain a fracture factor, more information in the form of availability of fracture surfaces and reactivity of fracture surfaces must be taken into account. Since references do not take these additional parameters into account, surface area measurements represent a total surface area instead of an effective surface area. The rationale for this assumption is that cracks created during cooling would be small, on the order of several tens of microns in width (Smith and Baxter 1981 [DIRS 102089], p. 15). It has been shown (Perez and Westsik 1981 [DIRS 111044]) that, even though total surface area may increase, fractures of this size have no appreciable contribution to the degradation rate of glass.

Confirmation status: This assumption is considered to be realistic. Therefore, no further confirmation is required.

Use in the analysis: This assumption is used in Appendix II (glass-fracturefac.xls) to calculate the fracture factor of high-level waste glass as presented in Table 6-16 and Table 6-17 in Section 6.4.3.1.

\subsubsection{Reactivity of Glass Fractures}

Assumption: It is also assumed that the reactivity of surfaces within fractures would be $1 / 2$ of the reactivity of unrestricted glass surfaces.

Rationale: Studies have shown that for highly fractured material (such as glass in a dropped canister), fractures display lower degradation rates than the outer surface area of the material. The degradation rate only increases slightly because fluid in the fractures will presumably not flow very freely and degradation will slow due to back reactions or precipitation. To be consistent with glass fracture reactivity used by Defense HLW Glass Degradation Model (BSC 2004 [DIRS 167619], Section 6.5.6), this analysis also uses $1 / 2$ for the glass fracture reactivity.

Confirmation status: This assumption is considered to be realistic. Therefore, no further confirmation is required.

Use in the analysis: This assumption is used in Appendix II (glass-fracturefac.xls) to calculate the fracture factor of high-level waste glass as presented in Table 6-16 and Table 6-17 in Section 6.4.3.1. 


\section{SCIENTIFIC ANALYSIS DISCUSSION}

Where possible, the empirical cumulative distribution function (ECDF) is used to describe the corrosion data. Given the limited number of data points for corrosion of materials, the underlying distribution is not obvious and, therefore, the ECDF is considered appropriate for use. D’Agostino and Stephens (1986 [DIRS 160320], Chapter 2) define ECDF for any random sample as:

$$
F_{n}(x)=\frac{\#\left(X_{j} \leq x\right)}{n},-\infty<x<\infty
$$

where the numerator is read as the number of samples $\left(X_{j}\right)$ less than or equal to $x$ (for this analysis, the corrosion value) with $n$ equaling the number of samples. The ECDF is a step function that estimates the population distribution function. Thus, for $F_{n}(x)$, as $x$ increases it takes a step up of height $1 / n$ as each sample observation is reached. The $\operatorname{ECDF}\left[F_{n}(x)\right]$ differs from the $\operatorname{CDF}[F(x)]$ in that it records the proportion of observations less than or equal to $x$ while the CDF represents the probability of an observation less than or equal to $x . F_{n}(x)$ can be used to estimate $F(x)$, and is a consistent estimator of $F(x)$ as $n$ approaches infinity.

The use of the ECDF plot does not depend upon assumptions concerning the underlying distribution and has several advantages over other statistical devices such as:

- It is invariant under monotone transformations with regard to quantities (however, the appearance may change)

- Its complexity is independent of the number of observations

- It supplies immediate and direct information regarding the shape of underlying distribution (i.e., skewness and bimodality)

- It is an effective indicator of peculiarities such as outliers

- It supplies robust information on location and dispersion

- It does not involve grouping difficulties such as would arise in a histogram.

One of the disadvantages to the use of the ECDF is that they can be sensitive to random occurrences in the data, especially when the data set is small. Another limitation encountered with the use of the ECDF is that if the user is interested in any value outside of the range of values used to create the ECDF, the probability is zero. However, all inputs used to create the ECDF plots in this analysis are presented in Section 4 and are archived in output DTN: MO0409SPAACRWP.000 and may be used to obtain a probability curve by the user.

Due to limited information or the nature of some data or populations, there are several materials for which the ECDF could not be used. The distributions for these cases are described in the following sections as they arise. 
The developed statistical parameters and ECDFs for aqueous degradation rates affecting waste package materials degradation will encompass various aqueous parameters such as temperature (up to $100^{\circ} \mathrm{C}$ ), water type (i.e., fresh versus saline), and $\mathrm{pH}$. Corrosion of materials at $\mathrm{pH}$ extremes (below 4 and above 10) is not included in this analysis since this is a special condition for which materials commonly display different corrosion behaviors. The output from this analysis is to be used in corrosion analysis to determine likelihood of corrosion scenarios and most likely corrosion rates for waste package materials to be used in corrosion studies.

The output from this analysis will support the determination of the probability of criticality for DOE SNF codisposal waste packages. Outputs can also be used for corrosion analyses to determine realistic (most probable) values of corrosion for various materials in waste packages.

\subsection{TYPES OF CORROSION}

The purpose of this report is not to describe the performance of engineered barriers for the TSPA-LA. Instead, the analysis provides simple statistics on aqueous corrosion rates of steels and alloys. In the EQ6 cases used to characterize corrosion of DOE waste packages, the rate is represented as a general corrosion over the entire surface of the material. For example, localized corrosion weight loss rates for aluminum are converted to general corrosion rates in $\mu \mathrm{m} / \mathrm{yr}$ for use in EQ6 calculations (BSC 2001 [DIRS 157640]; BSC 2002 [DIRS 158828]). Since the purpose of this analysis is to support EQ6 reaction path calculations and analyses, it will also focus on general corrosion rates. However, for some materials (such as aluminum), the primary corrosion behavior is a form of localized corrosion called pitting. In addition, galvanic coupling of materials in proximity to each other can have a strong effect on the rates of corrosion. Therefore, some data from specimens exhibiting localized or galvanic corrosion weight loss have been converted to general corrosion rates in $\mu \mathrm{m} / \mathrm{yr}$ and included in the rates presented in Section 6.2. This section presents a short overview of these corrosion types.

\subsubsection{General Corrosion}

General corrosion describes the process by which the entire surface of the metal is attacked uniformly.

\subsubsection{Localized Corrosion}

Localized corrosion of passive metals includes various phenomena such as pitting, crevice corrosion, intergranular attack, and stress corrosion cracking.

Crevice corrosion is a form of localized corrosion that can occur within crevices or shielded surfaces in which a solution can stagnate. Crevices can form from 1) the geometry of a structure (riveted plates, threaded joints, etc.), 2) Contact of the metal with nonmetallic solids (plastics, rubber, or glass associated with rivets, bolts, gaskets, welds, etc.), and 3) Deposits of sand, dirt, or corrosion products, or microbial growths on the metal surface (Shreir et al. 1998 [DIRS 100891], Section 1.6; Sedriks 1996 [DIRS 164036], Section 5). This corrosion can range from small pits to extensive corrosion over the whole surface. 
Pitting corrosion includes the formation of cavities within the passivated area. With this type of corrosion, the metal may be fully penetrated despite having a low general corrosion rate. Pitting may also range from a few deep holes in the metal to the metal surface being completely covered with pits of differing depth (Sedriks 1996 [DIRS 164036], Section 4).

Crevice and pitting corrosion depend on the stability of the passive film, the metal, the aqueous environment, and temperature. As a general rule, increasing temperature and halide content of the corrosive medium increase localized attack. As with general corrosion, the elements chromium, nickel, and molybdenum help decrease the corrosion rate during localized corrosion. Stainless steel is usually quite resistant to localized attack. Sedriks (1996 [DIRS 164036], p. 214) cites $300 \mathrm{ppm}$ as a safe chloride level for Stainless Steel Type 316, but other experiments indicate that halide levels can be much higher before localized corrosion becomes a problem. Carbon steel, lacking the alloying elements, is more susceptible. Aluminum alloys are very susceptible to pitting and usually display this behavior over long periods of exposure, in conditions causing the oxide film to break down. Rates for localized corrosion and depth of attack have been recorded by Gdowski and Bullen (1988 [DIRS 100860]), Sedriks (1996 [DIRS 164036]), Aziz (1956 [DIRS 159379]), and Kain et al. (1984 [DIRS 159385]).

\subsubsection{Galvanic Corrosion}

In a bimetallic couple, one metal (the anodic metal) corrodes sacrificially, protecting the cathode from corrosion. In most cases, this causes the corrosion rate of the anode to increase and the corrosion rate of the cathode to decrease or for corrosion to halt. Factors affecting the galvanic corrosion of a bimetallic couple include (Zhang 2000 [DIRS 164938], Figure 1):

- Reversible electrode potentials

- Reactions: dissolution, oxygen reduction, and hydrogen evolution

- Metallurgical factors: alloying, heat treatment, and mechanical working

- Surface conditions: surface treatment, passive film, and corrosion products

- Geometric factors: area; distance, position, shape, and orientation

- Environmental effects: forms of moisture, cyclic wet-dry, solar radiation, climate, and seasonal variations

- Electrolyte properties: ionic species, $\mathrm{pH}$, conductivity, temperature, volume, and flow rate.

Possible bimetallic partners inside waste packages and their relation to each other can be found in Table 6-1. 
Table 6-1. Major Bi-Metallic Couples Located Within Waste Packages

\begin{tabular}{|c|c|c|c|}
\hline Metal & Coupled With & Couple Anodic Member & Effect on Anode \\
\hline Aluminum & Carbon Steel & Aluminum $^{a}$ & Slight to marked increase in corrosion \\
\hline Aluminum & Stainless Steel & Aluminum $^{a}$ & Slight to marked increase in corrosion \\
\hline Aluminum & Aluminum & $\mathrm{N} / \mathrm{A}^{\mathrm{a}}$ & none \\
\hline Aluminum & Zircaloy & Aluminum $^{c}$ & Increase in corrosion \\
\hline Carbon Steel & Stainless Steel & Carbon Steel $^{a}$ & Marked increase in corrosion \\
\hline Carbon Steel & Carbon Steel & $\mathrm{N} / \mathrm{A}^{\mathrm{a}}$ & none \\
\hline Carbon Steel & Zircaloy & Zircaloy $^{\mathrm{b}}$ & Increase in corrosion \\
\hline Stainless Steel & Stainless Steel & $\mathrm{N} / \mathrm{A}^{\mathrm{a}}$ & none \\
\hline Stainless Steel & Zircaloy & Zircaloy $^{\mathrm{b}}$ & Increase in corrosion \\
\hline Stainless Steel & Alloy 22 & Stainless Steel $^{d}$ & Increase in corrosion $^{d}$ \\
\hline
\end{tabular}

Sources: ${ }^{a}$ Shreir et al. 1998, Table 1.25 [DIRS 100891].

${ }^{\mathrm{b}}$ Cole 1976 [DIRS 159369] and Smith 1987 [DIRS 159375]; Zr degrades sacrificially in the presence of stainless steel and ferric iron.

'Yau and Webster 1987 [DIRS 165063].

NOTE: $\quad{ }^{d} A$ typical galvanic series indicates that the stainless steels are less noble than Hastalloy Alloy C, which is similar to Alloy 22.

\subsection{Steels and Alloys}

As presented in Section 4.1.3, corrosion data of steels and alloys is divided according to the type of corroding medium and, where appropriate, temperature. The freshwater rates are representative of those solutions, which are dilute, such as lake water and J-13 well water. The saltwater in this case is ocean water with an average chloride content of 17,115 to 17,357 ppm (Forgeson et al. 1958 [DIRS 159343]) to simulate natural waters that have been concentrated by evaporation or contact with engineered materials. Any additions or modification to these definitions will be discussed below with the specific materials.

For solutions indicated by SDW, SCW, and SAW, this is indicative of Simulated Dilute Well Water, Simulated Concentrated Well Water, and Simulated Acidified Well Water (McCright 1998 [DIRS 114637]). The SDW simulates J-13 well water at $10 \times$ concentration to account for minor effects of water evaporation and boiling, while SCW simulates J-13 well water concentrated $1,000 \times$ to account for long-term water evaporation and boiling in the repository environment. The SAW represents J-13 well water that has been acidified and concentrated to simulate microbial metabolic products. Further information on these simulated solutions can be found in Corrosion Data and Modeling, Update for Viability Assessment (McCright 1998 [DIRS 114637], Section 2.2.4).

Compositions of alloys used in the analysis of corrosion rates are located in Table 6-2. 
Table 6-2. Composition (wt \%) of Steel and Alloys

\begin{tabular}{|c|c|c|c|c|c|c|c|c|c|c|c|c|c|c|c|c|c|c|}
\hline $\begin{array}{l}\text { Density } \\
\left(\mathrm{g}_{\mathrm{g}} \mathrm{cm}^{3}\right)\end{array}$ & Material & C & $\mathrm{Mn}$ & $\mathbf{P}$ & $\mathrm{Cu}$ & $s$ & $\mathrm{Fe}$ & $\mathrm{Si}$ & $\mathrm{Cr}$ & $\mathrm{Ni}$ & $\mathbf{N}$ & Al & Mo & $\mathrm{Mg}$ & $\mathrm{Zn}$ & $\mathrm{Ti}$ & Other & Total \\
\hline $304 \mathrm{~L}=7.94^{\mathrm{b}}$ & $302^{\mathrm{a}}$ & 0.15 & 2.00 & 0.045 & - & 0.030 & $\mathrm{Bal}$ & 1.00 & 17.0 to 19.0 & 8 to 10 & 0.1 & - & - & - & - & - & - & - \\
\hline $304 \mathrm{~L}=7.94^{\mathrm{b}}$ & $304^{a}$ & 0.08 & 2.00 & 0.045 & - & 0.030 & Bal & 1.00 & 18.0 to 20.0 & 8 to 11 & - & - & - & - & - & - & - & - \\
\hline $304 \mathrm{~L}=7.94^{\mathrm{b}}$ & $304 \mathrm{~L}^{\mathrm{a}}$ & 0.030 & 2.00 & 0.045 & - & 0.030 & Bal & 1.00 & 18.0 to 20.0 & 8 to 12 & - & - & - & - & - & - & - & - \\
\hline $\mathrm{N} / \mathrm{A}$ & B-304L Poison ${ }^{\mathrm{C}}$ & 0.030 & 1.95 & 0.04 & - & 0.03 & $\mathrm{Bal}$ & 0.73 & 18.56 & 9.77 & 0.10 & - & - & - & - & - & $2.33 \mathrm{~B}$ & - \\
\hline N/A & $825^{d}$ & 0.02 & 0.37 & - & 1.90 & 0.004 & Bal & 0.08 & 22.70 & 43.98 & - & 0.06 & 2.92 & - & - & 1.07 & - & - \\
\hline $316 \mathrm{~L}=7.98^{\mathrm{b}}$ & $316^{a}$ & 0.08 & 2.00 & 0.045 & - & 0.030 & $\mathrm{Bal}$ & 1.00 & 16.0 to 18.0 & 10 to 14 & - & - & 2.00 to 3.00 & - & - & - & - & - \\
\hline $316 \mathrm{~L}=7.98^{\mathrm{b}}$ & $316 \mathrm{~L}^{\mathrm{a}}$ & 0.030 & 2.00 & 0.045 & - & 0.030 & $\mathrm{Bal}$ & 1.00 & 16.0 to 18.0 & 10 to 14 & - & - & 2.00 to 3.00 & - & - & - & - & - \\
\hline $316 \mathrm{~L}=7.98^{\mathrm{b}}$ & $316 \mathrm{NG}^{\mathrm{n}}$ & 0.020 & 2.00 & 0.045 & - & 0.030 & Bal & 1.00 & 16.0 to 18.0 & 10 to 14 & 0.06 to 0.10 & - & 2.00 to 3.00 & - & - & - & - & - \\
\hline $7.76^{\mathrm{e}}$ & Neutronit $(\text { A978) })^{f}$ & 0.04 & - & - & - & - & Bal & - & 18.5 & 13 & - & - & 2.2 & - & - & - & \multicolumn{2}{|c|}{$0.20 \mathrm{Co} / 1.245 \mathrm{E}$} \\
\hline 7.90 & $\mathrm{AM}-350^{\circ}$ & 0.07 to 0.11 & 0.5 to 1.25 & 0.04 & - & 0.03 & Bal & 0.50 & 16.0 to 17.0 & 4.0 to 5.0 & 0.07 to 0.13 & - & 2.5 to 3.25 & - & - & - & - & - \\
\hline N/A & $\mathrm{A} 516^{\mathrm{m}}$ & 0.28 & 0.85 to 1.20 & 0.035 & - & 0.035 & Bal & 0.15 to 0.40 & - & - & - & - & - & - & - & - & - & - \\
\hline $\mathrm{N} / \mathrm{A}$ & Alloy N06464 ${ }^{\mathrm{p}}$ & $0.010 \max$ & $0.5 \max$ & $0.005 \max$ & - & $0.005 \max$ & $1.0 \max$ & $0.08 \max$ & 14.5 to 17.1 & Bal. & $0.010 \max$ & - & 13.1 to 16.0 & - & - & - & \multicolumn{2}{|c|}{$\begin{array}{c}2.0 \mathrm{max} \mathrm{Co} \\
1.9 \text { to } 2.1 \mathrm{Gd} \\
0.005 \mathrm{O}\end{array}$} \\
\hline $\mathrm{N} / \mathrm{A}$ & $24 \mathrm{~S}-\mathrm{T} 3^{9}$ & - & 0.58 & - & 4.54 & - & 0.30 & 0.15 & - & - & - & $\mathrm{Bal}$ & - & 1.46 & - & - & - & - \\
\hline N/A & $\begin{array}{l}\text { Alclad 24S-T3 } \\
\text { Coating }^{9}\end{array}$ & - & 0.01 & - & 0.27 & - & 0.13 & 0.07 & - & - & - & Bal & - & 0.13 & - & - & - & - \\
\hline N/A & $\begin{array}{l}\text { Alclad 24S-T3 } \\
\text { Core }^{g}\end{array}$ & - & 0.56 & - & 4.19 & - & 0.28 & 0.11 & - & - & - & Bal & - & 1.39 & - & - & - & - \\
\hline $\mathrm{N} / \mathrm{A}$ & $52 \mathrm{~S}-1 / 2 \mathrm{H}^{9}$ & - & 0.05 & - & 0.02 & - & 0.15 & 0.12 & 0.21 & - & - & $\mathrm{Bal}$ & - & 2.53 & - & - & - & - \\
\hline N/A & $\begin{array}{c}\text { Alclad 75S-T6 } \\
\text { Coating }^{9}\end{array}$ & - & 0.01 & - & 0.09 & - & 0.36 & 0.07 & & - & - & Bal & - & 0.15 & 1.31 & - & - & - \\
\hline N/A & $\begin{array}{l}\text { Alclad 75S-T6 } \\
\text { Core }^{g}\end{array}$ & - & 0.12 & - & 1.58 & - & 0.15 & 0.08 & 0.24 & - & - & Bal & - & 2.61 & 5.9 & 0.05 & - & - \\
\hline N/A & $3 \mathrm{~S}-1 / 2 \mathrm{H}^{\mathrm{h}}$ & - & 1.2 & - & - & - & - & - & - & - & - & Bal & - & - & - & - & - & - \\
\hline N/A & Alclad 3S-1/2H & \multicolumn{17}{|c|}{ Unavailable } \\
\hline $\mathrm{N} / \mathrm{A}$ & $61 \mathrm{~S}-\mathrm{T}^{\mathrm{h}}$ & - & - & - & 0.25 & - & - & 0.6 & 0.25 & - & - & Bal & - & 1.0 & - & - & - & - \\
\hline $2.71^{b}$ & $1100-\mathrm{H} 14^{i}$ & - & 0.05 & - & 0.05 to 0.20 & - & \multicolumn{2}{|c|}{$0.95(\mathrm{Fe}+\mathrm{Si})$} & - & - & - & - & - & - & 0.10 & - & 0.05 & 0.15 \\
\hline $2.73^{b}$ & $3003-\mathrm{H} 14^{i}$ & - & 1.0 to 1.5 & - & 0.05 to 0.20 & - & 0.7 & 0.6 & - & - & - & Bal & - & - & 0.10 & - & 0.05 & 0.15 \\
\hline $2.68^{\mathrm{b}}$ & $5052-\mathrm{H} 34^{i}$ & - & 0.10 & - & 0.10 & - & 0.4 & 0.25 & 0.15 to 0.35 & - & - & Bal & - & 2.2 to 2.8 & 0.10 & - & 0.05 & 0.15 \\
\hline $2.68^{r}$ & $5056^{j}$ & - & 0.05 to 0.20 & - & 0.10 & - & 0.4 & 0.3 & 0.05 to 0.20 & - & - & Bal & - & 4.5 to 5.6 & 0.10 & - & 0.05 & 0.15 \\
\hline $2.66^{\mathrm{b}}$ & $5083^{i}$ & - & 0.40 to 1.0 & - & 0.1 & - & 0.4 & 0.4 & 0.05 to 0.25 & - & - & Bal & - & 4.0 to 4.9 & 0.25 & 0.15 & 0.05 & 0.15 \\
\hline $2.66^{\mathrm{b}}$ & $5154^{i}$ & - & 0.10 & - & 0.10 & - & 0.4 & 0.25 & 0.15 to 0.35 & - & - & Bal & - & 3.1 to 3.9 & 0.20 & 0.20 & 0.05 & 0.15 \\
\hline $2.70^{\mathrm{s}}$ & 6051-T4 & \multicolumn{17}{|c|}{ Unavailable } \\
\hline $2.70^{\mathrm{s}}$ & 6051-T6 & \multirow{2}{*}{\multicolumn{17}{|c|}{$\begin{array}{l}\text { Unavailable } \\
\text { Unavailable }\end{array}$}} \\
\hline $2.70^{\mathrm{s}}$ & 6053-T6 & & & & & & & & & & & & & & & & & \\
\hline $2.70^{\mathrm{b}}$ & $6061-\mathrm{T}^{\mathrm{i}}$ & - & 0.15 & - & 0.15 to 0.40 & - & 0.7 & \begin{tabular}{|l|}
0.40 to 0.8 \\
\end{tabular} & 0.04 to 0.35 & - & - & Bal & - & 0.8 to 1.2 & 0.25 & 0.15 & 0.05 & 0.15 \\
\hline $2.70^{\mathrm{b}}$ & 6061-T6 & - & 0.15 & - & 0.15 to 0.40 & - & 0.7 & 0.40 to 0.8 & 0.04 to 0.35 & - & - & Bal & - & 0.8 to 1.2 & 0.25 & 0.15 & 0.05 & 0.15 \\
\hline $2.81^{\mathrm{t}}$ & $7072^{i}$ & - & 0.10 & - & 0.10 & - & $0.7(\mathrm{Fe}+\mathrm{Si})$ & - & - & - & & $\mathrm{Bal}$ & - & 0.10 & 0.8 to 1.3 & - & 0.05 & 0.15 \\
\hline $2.81^{b}$ & 7075-T6' & - & 0.30 & - & 1.2 to 2.0 & - & 0.50 & 0.40 & 0.18 to 0.28 & - & - & $\mathrm{Bal}$ & - & 2.1 to 2.9 & 5.1 to 6.1 & 0.20 & 0.05 & 0.15 \\
\hline $2.73^{u}$ & 3003-Alcladi & \multicolumn{17}{|c|}{ Nloy Type 7072} \\
\hline $2.70^{u}$ & 6061-Alcladi & \multirow{2}{*}{\multicolumn{17}{|c|}{ Aluminum Alloy Type 6061 clad with Aluminum Alloy Type 7072}} \\
\hline $2.81^{u}$ & 7075-Alclad' & & & & & & & & & & & & & & & & & \\
\hline
\end{tabular}


Table 6-2. Composition (wt \%) of Steel and Alloys (Continued)

\begin{tabular}{|c|c|c|c|c|c|c|c|c|c|c|c|c|c|c|c|c|c|c|}
\hline $\begin{array}{l}\text { Density } \\
\left(\mathrm{g} / \mathrm{cm}^{3}\right)\end{array}$ & Material & c & $\mathrm{Mn}$ & $\mathbf{P}$ & $\mathrm{Cu}$ & $\mathbf{s}$ & $\mathrm{Fe}$ & $\mathrm{Si}$ & $\mathrm{Cr}$ & $\mathrm{Ni}$ & $\mathbf{N}$ & Al & Mo & $\mathrm{Mg}$ & $\mathrm{Zn}$ & $\mathrm{Ti}$ & Other & Total \\
\hline $2.71^{9}$ & Alcan $2 \mathrm{~S}-\mathrm{O}^{\mathrm{k}}$ & - & 0.01 & - & 0.01 & - & 0.36 & 0.22 & No data & - & - & Bal & - & No data & - & 0.01 & - & - \\
\hline N/A & Inconel $600^{\prime}$ & 0.15 & 1.0 & - & 0.5 & 0.015 & 6.0 to 10.0 & 0.5 & 14.0 to 17.0 & $72 \mathrm{~min}$ & - & - & - & - & - & - & - & - \\
\hline N/A & Inconel $625^{1}$ & 0.1 & 0.50 & 0.015 & - & 0.015 & 5.0 & 0.5 & 20.0 to 23.0 & $58.0 \mathrm{~min}$ & - & 0.40 & 8.0 to 10.0 & - & - & 0.40 & \multicolumn{2}{|c|}{$\begin{array}{c}3.15 \text { to } 4.15 \\
\mathrm{Nb}+\mathrm{Ta} \text { and } 1.0 \mathrm{Co} \\
\end{array}$} \\
\hline $\mathrm{N} / \mathrm{A}$ & Inconel X-750' & 0.08 & 1.00 & - & 0.50 & 0.01 & 5.0 to 9.0 & 0.50 & 14.0 to 17.0 & $70.0 \mathrm{~min}$ & - & 0.4 to 1.00 & - & - & - & 2.25 to 2.75 & \multicolumn{2}{|c|}{$\begin{array}{c}0.70 \text { to } 1.20 \mathrm{Nb}, \\
1.00 \mathrm{Co}\end{array}$} \\
\hline $\mathrm{N} / \mathrm{A}$ & Titanium Grade $7^{v}$ & $0.08 \max$ & - & - & - & - & $0.30 \max$ & - & - & - & $0.03 \max$ & - & - & - & - & Bal & \multicolumn{2}{|c|}{$\begin{array}{c}0.015 \mathrm{max} \mathrm{H}, \\
0.25 \mathrm{max} \mathrm{O} \text {, and } \\
0.12 \text { to } 0.25 \mathrm{Pd}\end{array}$} \\
\hline N/A & Titanium Grade $24^{v}$ & $0.08 \max$ & - & - & - & - & $0.40 \max$ & - & - & - & $0.05 \max$ & 5.5 to 6.75 & - & - & - & Bal & \multicolumn{2}{|c|}{$\begin{array}{c}0.015 \mathrm{max} \mathrm{H} \\
0.20 \mathrm{max} \mathrm{O} \\
3.5 \text { to } 4.5 \mathrm{~V} \text {, and } \\
0.04 \text { to } 0.08 \mathrm{Pd}\end{array}$} \\
\hline
\end{tabular}

Sources: aASTM A 276-03 [DIRS 165006].

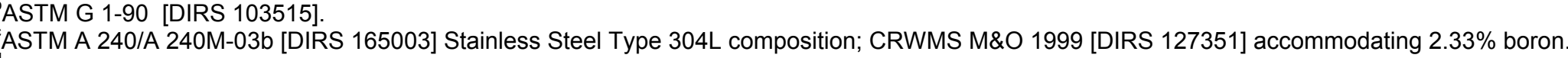

${ }^{\mathrm{d}}$ Beavers et

DTN: MO0109RIB00049.001 IRTS 155964

'DTN: MO0109RIB00049.001 [DIRS 155964]; ASTM A $887-89$ [DIRS 154062], Table 1, S30463, S30464, and S30466 for boron content.

BBomberger et al. 1954 [DRS 163599$]$.

Sawyer and Brown 1947 [DIRS 164921 .

Ailor 1969 [DIRS 164907] Table 11.

Aziz 1956 [DIRS 159379].

Anco Alloys International 1988 [DIRS 130835 .

ASTM A56/A 51GM 2001 [DIRS 162723] (Table 1, 2" to 4" thickness, Grade 65)

Danko 1987 [DIRS 15439001, p. 931 - Stainless Stel 1 .

VASTM B 265-02 2002 [DIRS 162726].

NOTES: ${ }^{9}$ Assumed the same as Aluminum Alloy 1100 (Section 5.2.2).

'Assumed the same as Aluminum Alloy 5052 (Section 5.2.2).

Assumed the same as Aluminum Alloy 6061 (Section 5.2.2.

Assumed the same as Aluminum Alloy 7075 (Section 5.2.2).
"Assumed same density as aluminum-alloy comprising core (Section 5.2 .2 ). 


\subsubsection{Stainless Steel}

The extent to which corrosion proceeds is highly dependent on the elemental composition of the material. Hudson and Stanners (1955 [DIRS 159334]) show that even small amounts of chromium will slow damage to steel. Even in highly oxidizing environments, chromium lends strong resistance to attack (Scarberry et al. 1967 [DIRS 159335]). The addition of nickel further fortifies the steel, as it is effective in reducing corrosion in reducing conditions and in neutral salt solutions and alkalies (Scarberry et al. 1967 [DIRS 159335]). The introduction of molybdenum to stainless steel is done to aid in corrosion resistance, but specifically, to add resistance to localized corrosion (Scarberry et al. 1967 [DIRS 159335]). However, the addition of molybdenum can have a negative effect in highly oxidizing conditions (Wallen and Olsson 1977 [DIRS 164948]).

Stainless steels planned for use in the waste packages (Stainless Steel Type 304 series and 316 series) contain significant amounts of chromium, nickel, and molybdenum (ASTM A 240/A 240M-03b [DIRS 165003], ASTM A 276-03 [DIRS 165006], and Bauccio 1993 [DIRS 131480]). These three elements have been shown to significantly decrease the corrosion of stainless steel in aqueous environments ranging from mild freshwaters to caustic saltwater. This is accomplished through the formation of a passive layer (film). The passive films of stainless steels are very thin hydrous oxides enriched in chromium, silicon, and molybdenum. At high temperatures a more stable chromium-nickel spinel structure can form. By solid-state diffusion through the passive film, continuous dissolution and precipitation of the film occurs (Wallen and Olsson 1977 [DIRS 164948]; McCright et al. 1987 [DIRS 159336]) providing a protective barrier to attack from the surrounding media. Passive film formation causes the continual decrease of the degradation rate seen in many studies (i.e., Gdowski and Bullen 1988 [DIRS 100860]; Larrabee 1953 [DIRS 159337]). As time passes, unless otherwise disturbed, the passive film stabilizes and sometimes (in the case of freshwater corrosion) can halt corrosion of the steel completely.

Breakdown of the passive film can occur in highly oxidizing or reducing environments, in the presence of chloride or sulfates, and harsh, localized environments caused by physical properties such as galvanic coupling, surface blemishes on the material, and the growth of bacterial colonies. These effects usually contribute to forms of corrosion other than the general corrosion discussed here (discussion of localized and galvanic corrosion is in Sections 6.1.2 and 6.1.3).

As shown in the tables in Section 4.1.3, most of the corrosion data is from short-term experiments ranging from a few weeks to sixteen years. It has been generally shown that there is a continual decrease of the degradation rate over time (Gdowski and Bullen 1988 [DIRS 100860]; Larrabee 1953 [DIRS 159337]; and Southwell et al. 1976 [DIRS 100927]). Therefore, the rates may eventually reach a steady state in which the overall corrosion approaches $0 \mu \mathrm{m} / \mathrm{yr}$. Since corrosion rates over longer periods have not been measured, the data from short-term experiments will be used here to describe the corrosion of materials. Given the information above, the rates are expected to be low. The corrosion rates for Stainless Steel Types $304 \mathrm{~L}$ and $316 \mathrm{~L}$ in fresh or dilute waters expected at the repository can be found in Table 4-2 and Table 4-3. 
Another factor affecting stainless steel corrosion is the concentration of halides in the corroding medium. The chloride $\left(\mathrm{Cl}^{-}\right)$and fluoride $\left(\mathrm{F}^{-}\right)$content of several water types is presented in Table 6-3.

Table 6-3. Halide Content of Several Waters from Yucca Mountain Strata Compared to J-13 Well Water

\begin{tabular}{|c|c|c|c|c|c|}
\hline $\begin{array}{c}\text { Water } \\
\text { Type }\end{array}$ & $\begin{array}{c}\text { J-13 Well } \\
\text { Water }^{\mathbf{a}}\end{array}$ & $\begin{array}{c}\text { Tptpll Crown } \\
\text { Seepage }^{\mathbf{b}}\end{array}$ & $\begin{array}{c}\text { Evaporated } \\
\text { Tptpmn Crown } \\
\text { Seepage Water }^{\mathbf{c}}\end{array}$ & $\begin{array}{c}\text { Tptpll Crown } \\
\text { Seepage / Grout } \\
\text { Leachate }^{\mathbf{c}}\end{array}$ & $\begin{array}{c}\text { Evaporated Perched Water / } \\
\text { Grout Leachate }^{\mathbf{d}}\end{array}$ \\
\hline $\mathrm{Cl}^{-}(\mathrm{ppm})$ & 7.14 & 117 & 7,587 & 117 & 1,858 \\
\hline $\mathrm{F}^{-}(\mathrm{ppm})$ & 2.18 & 5.81 & 6.63 & 5.97 & None Reported \\
\hline
\end{tabular}

Sources: ${ }^{a}$ DTN:MO0006J13WTRCM.000 [DIRS 151029].

${ }^{b}$ BSC 2001 [DIRS 155859], Transition to ambient temperature, Table 11.

${ }^{\mathrm{C} B S C} 2001$ [DIRS 156183], Transition to ambient temperature, Tables 6.1-6 and 6.2-10.

${ }^{d}$ BSC 2001 [DIRS 156183], Ambient temperature, Table 6.2-16.

NOTE: All values converted to ppm in ppm_.xls on Appendix II.

For comparison, an example of the $\mathrm{Cl}^{-}$concentration in seawater at the Panama Canal Zone lies between 17,357 and 17,415 ppm (Forgeson et al. 1958 [DIRS 159343]). These components inside the corroding medium are important because they interfere with the maintenance of the passive film (Wallen and Olsson 1977 [DIRS 164948]; Davison et al. 1987 [DIRS 162971]) and accelerate localized corrosion of the metal. General corrosion rates for Stainless Steel Types 304L and 316L in saltwater used for this analysis are found in Table 4-4 and Table 4-5.

As indicated in Section 4.1.3, the corrosion resistance of stainless steels in freshwater and saltwater differs quite markedly. Therefore, they have been analyzed separately here.

\subsubsection{Stainless Steel Types 302/304/304L}

Probabilities for general corrosion rates of Stainless Steel Type 304L are presented in Degradation of Stainless Steel Structural Material (BSC 2001 [DIRS 156356]). However, these rates come primarily from data on the corrosion of stainless steel in seawater. As the waters entering the drift at Yucca Mountain will be dilute solutions (similar to J-13 well water, DTN: MO0006J13WTRCM.000 [DIRS 151029]), it is not expected that the corrosion rates of waste package materials will be as fast as those occurring in sea water. For this reason, the full suite of information of corrosion in fresh and saltwater, including time duration, and environmental conditions of experiments is presented in Section 4.1.3.1.

The statistical information on the corrosion of Stainless Steel Type 304L is located in Table 6-4 and Figure 6-1 and Figure 6-2. 
Freshwater Corrosion Rate $(\mu \mathrm{m} /$ year)

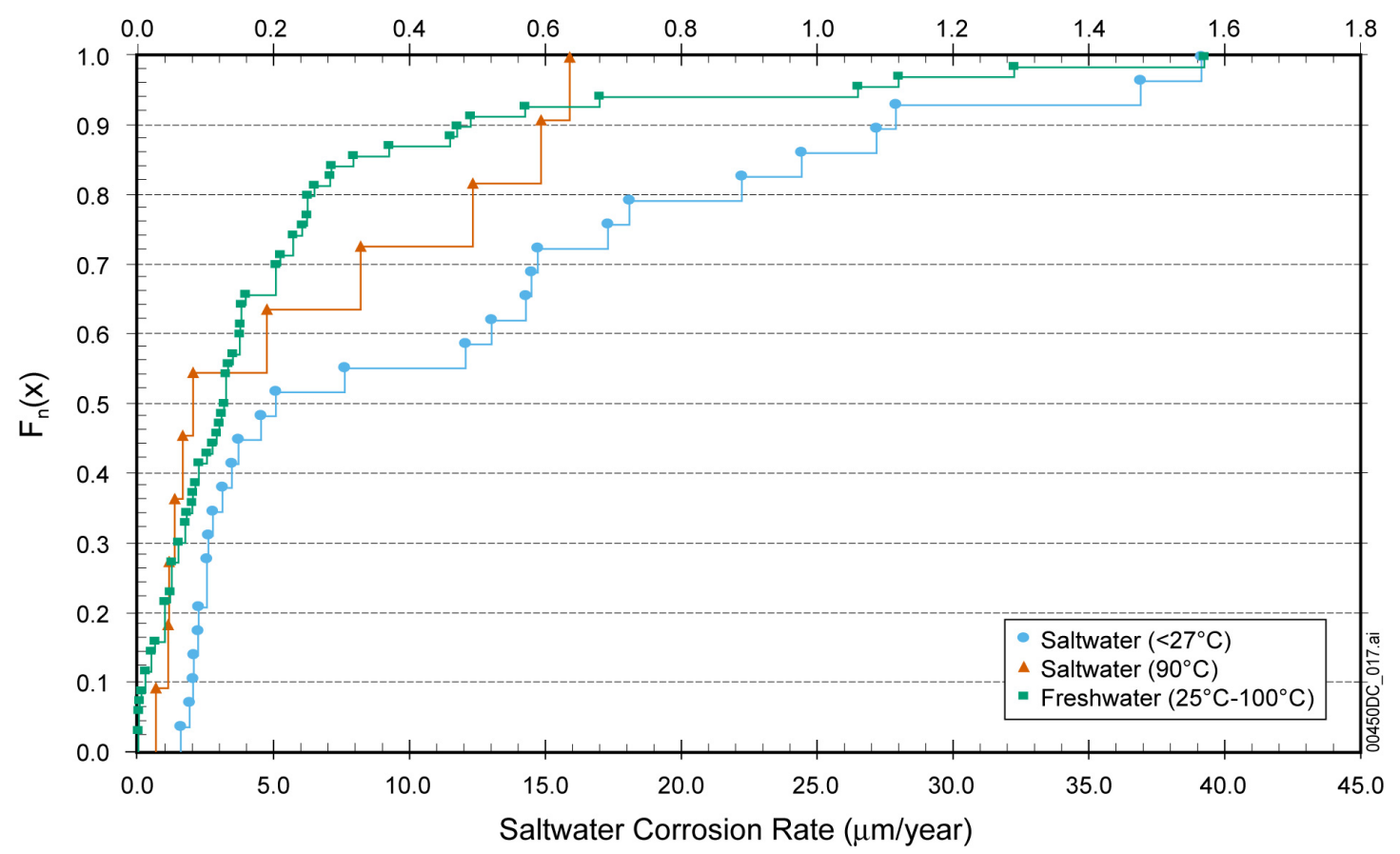

NOTE: $\quad$ ECDF calculated in ECDF_metals.xls in Appendix II from values in Table 4-2, Table 4-2, and Table 4-4. Archived in output DTN: MO0409SPAACRWP.000.

Figure 6-1. ECDF for Stainless Steel Types 302/304/304L in All Water Types Investigated

\section{Freshwater Corrosion Rate ( $\mu \mathrm{m} /$ year)}

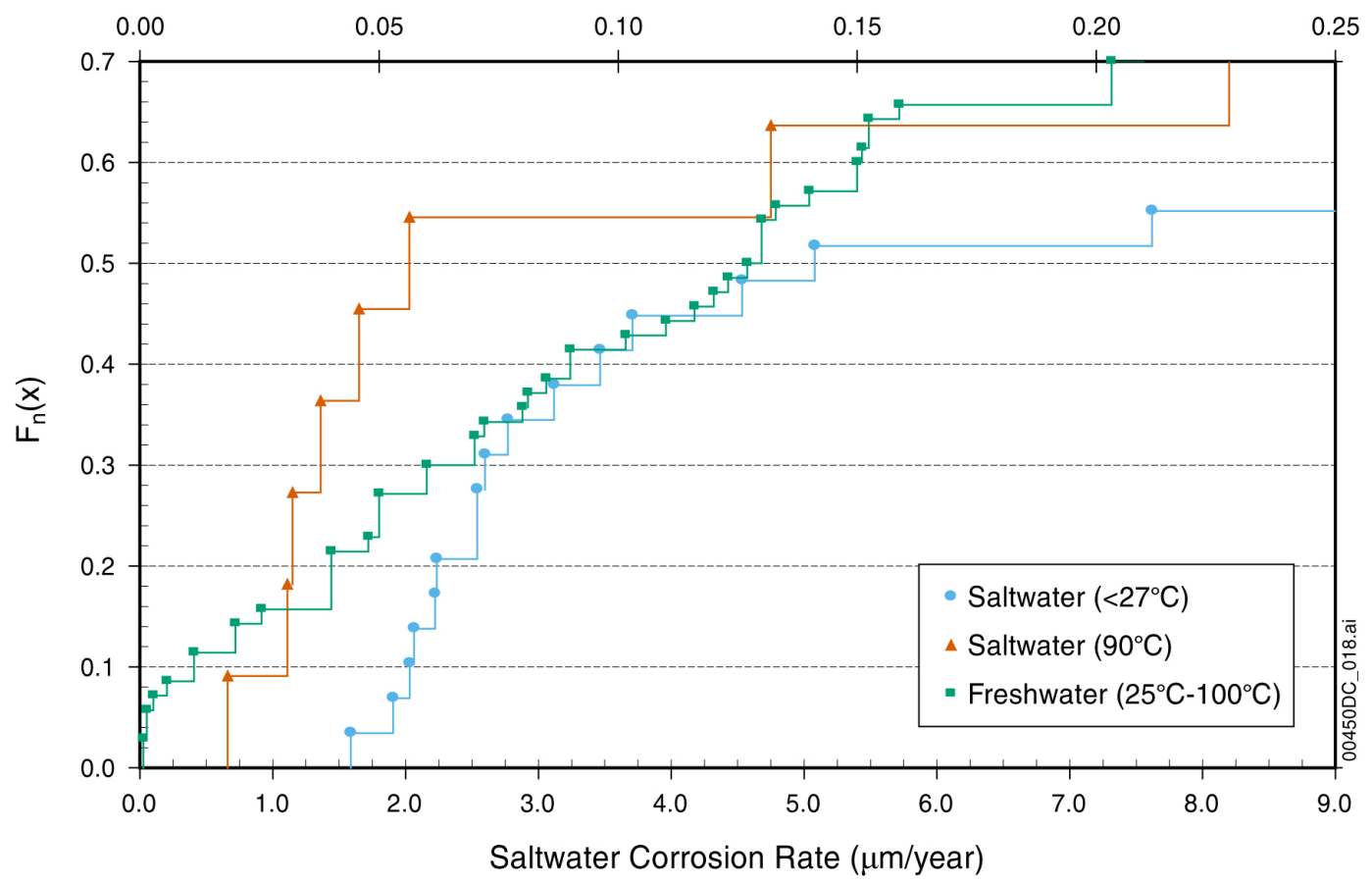

NOTE: $\quad$ ECDF calculated in ECDF_metals.xls in Appendix II from values in Table 4-2 and Table 4-4. Archived in output DTN: MO0409SPAACRWP.000.

Figure 6-2. ECDF for Stainless Steel Types 302/304/304L Showing Detail for Slower Rates 
Table 6-4. Minimum, Maximum, Mean, and Median Aqueous Corrosion Rates of Stainless Steel Types 302/304/304L

\begin{tabular}{|l|c|c|c|c|c|}
\hline \multirow{2}{*}{ Environment } & \multicolumn{4}{|c|}{ Corrosion Rate $(\mu \mathrm{m} / \mathbf{y r})$} & Standard \\
\cline { 2 - 6 } & Minimum & Maximum & Median & Mean & 0.298 \\
\hline $\begin{array}{l}\text { Freshwater }\left(25^{\circ} \mathrm{C}\right. \\
\left.\text { to } 100^{\circ} \mathrm{C}\right)\end{array}$ & 0.001 & 1.570 & 0.1285 & 0.214 & 11.134 \\
\hline Saltwater $\left(26.7^{\circ} \mathrm{C}\right)$ & 1.588 & 39.147 & 5.08 & 11.441 & 5.953 \\
\hline Saltwater $\left(90^{\circ} \mathrm{C}\right)$ & 0.660 & 15.900 & 2.03 & 5.816 & \\
\hline
\end{tabular}

NOTE: Calculated in aqueous-304L.x/s in Appendix II from values in Table 4-2 and Table 4-4. Archived in output DTN: MO0409SPAACRWP.000.

\subsubsection{Stainless Steel Types 316/316L/AM-350}

Probabilities for general corrosion rates of Stainless Steel Type 316L are also presented in Degradation of Stainless Steel Structural Material (BSC 2001 [DIRS 156356]). However, as for Stainless Steel Type 304L, these rates come primarily from data on the corrosion of stainless steel in seawater. As the waters entering the drift at Yucca Mountain will be dilute solutions (similar to J-13 well water, DTN: MO0006J13WTRCM.000 [DIRS 151029]), it is not expected that the corrosion rates of waste package materials will be as fast as those occurring in sea water. For this reason, the full suite of information of corrosion in fresh and saltwater, including time duration, and environmental conditions of experiments is presented Section 4.1.3.1.

The statistical information on the corrosion of Stainless Steel Type 316L is located in Table 6-5 and Figure 6-3 through Figure 6-5. 


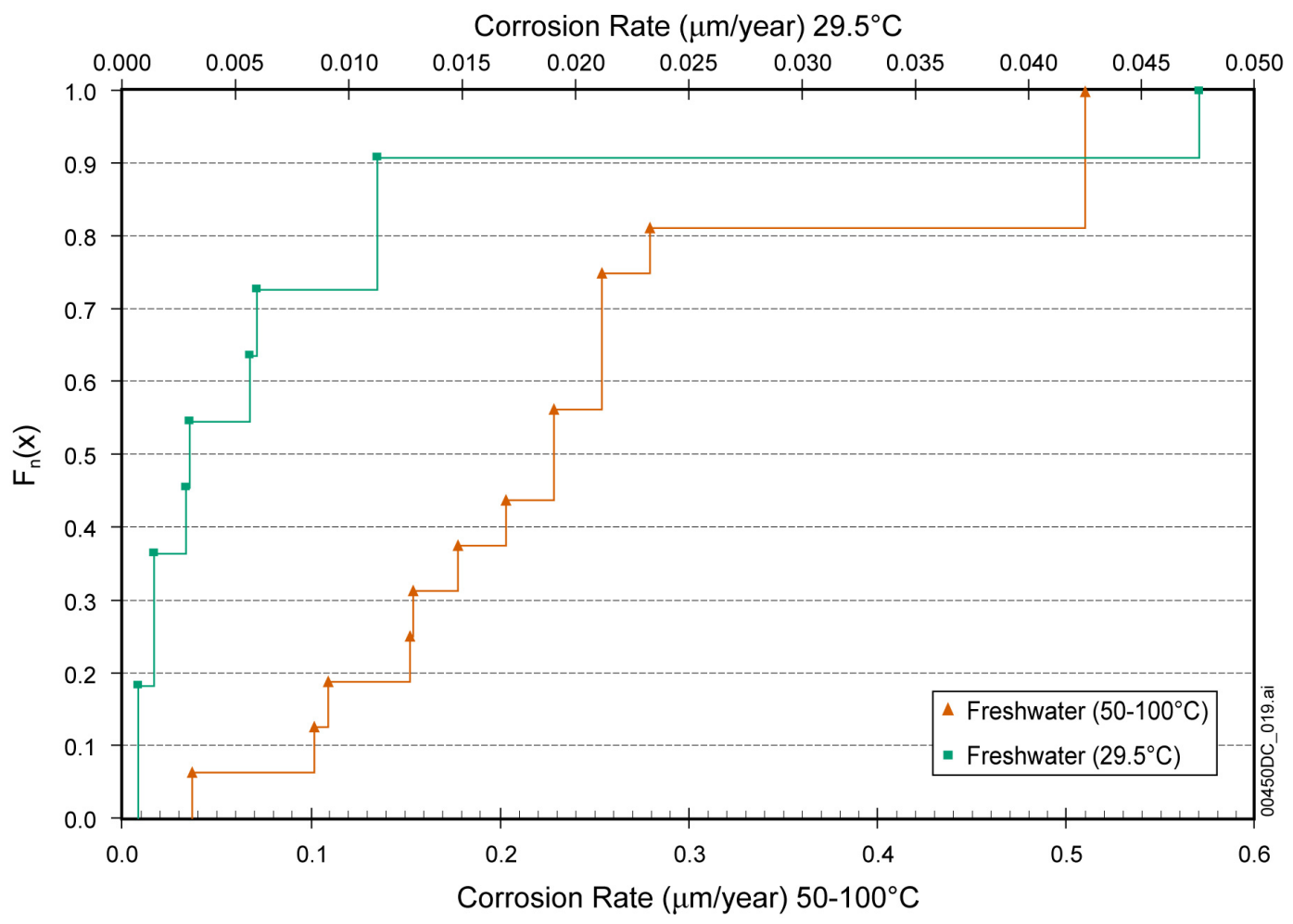

NOTE: $\quad$ ECDF calculated in ECDF_metals.x/s in Appendix II from values in Table 4-3. Archived in output DTN: MO0409SPAACRWP.000.

Figure 6-3. ECDF for Stainless Steel Types 316/316L/AM-350 in Freshwater

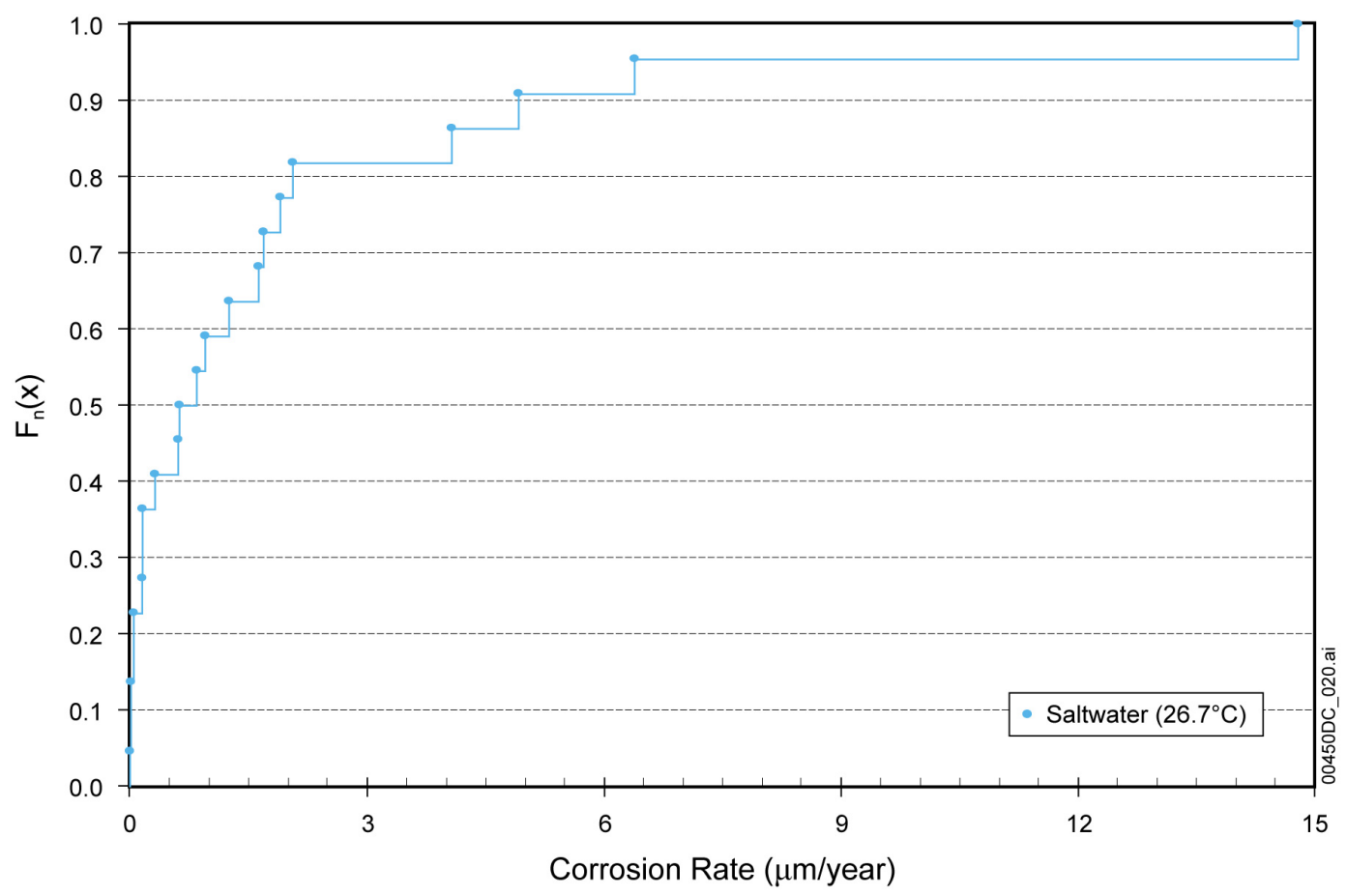

NOTE: $\quad$ ECDF calculated in ECDF_metals.xls in Appendix II from values in Table 4-5. Archived in output DTN: MO0409SPAACRWP.000.

Figure 6-4. ECDF for Stainless Steel Types 316/316L/AM-350 in Saltwater 


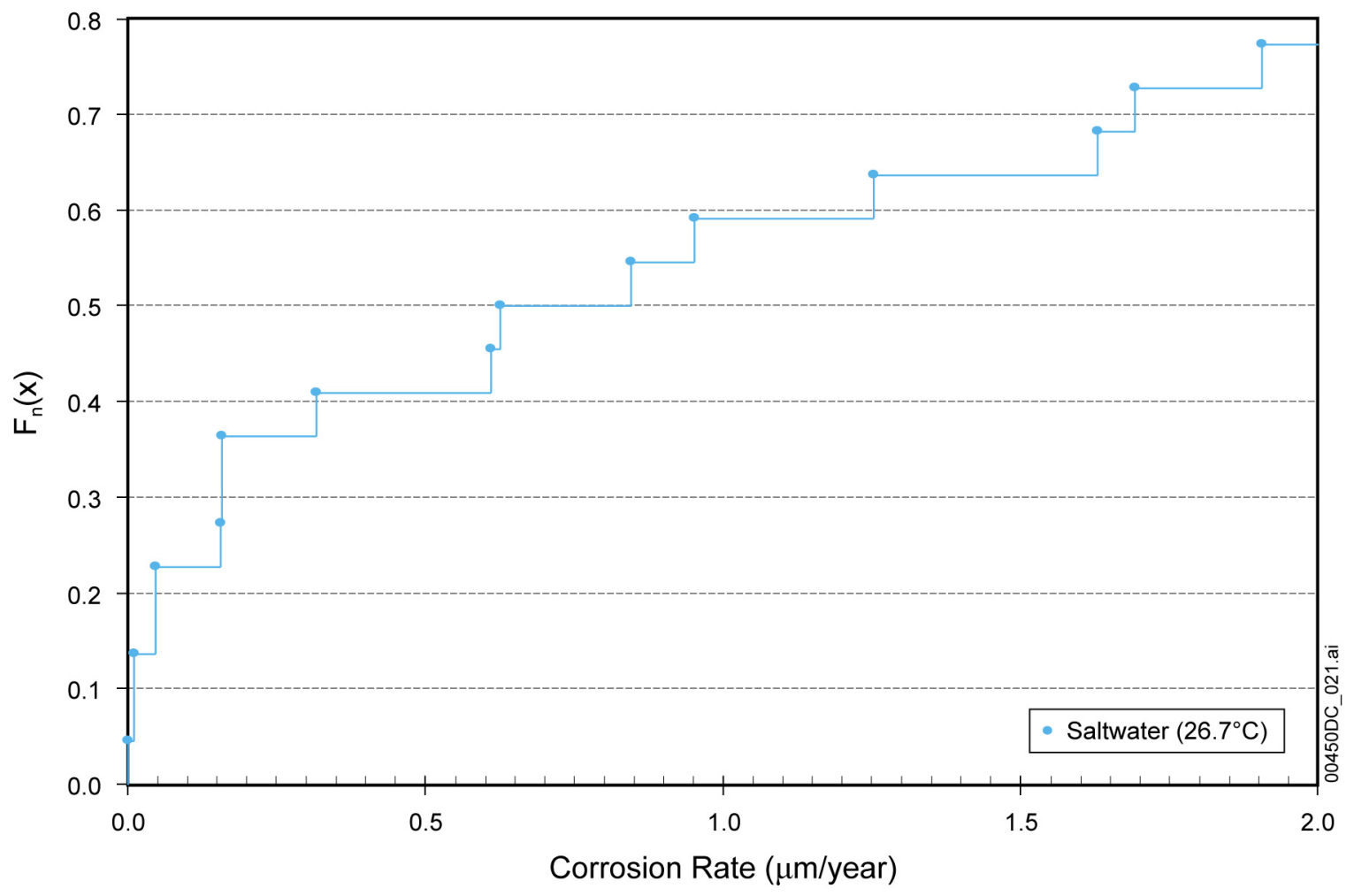

NOTE: $\quad$ ECDF calculated in ECDF_metals.x/s in Appendix II from values in Table 4-5. Archived in output DTN: MO0409SPAACRWP.000.

Figure 6-5. ECDF for Stainless Steel Types 316/316L/AM-350 in Saltwater Showing More Detail for Corrosion Rates Below $2.5 \mu \mathrm{m} / \mathrm{yr}$

Table 6-5. Minimum, Maximum, Mean, and Median Aqueous Corrosion Rates of Stainless Steel Types 316/316L/AM-350

\begin{tabular}{|l|c|c|c|c|c|}
\hline \multirow{2}{*}{ Environment } & \multicolumn{4}{|c|}{ Corrosion Rate $(\mu \mathrm{m} / \mathrm{yr})$} & \multirow{2}{*}{$\begin{array}{c}\text { Standard } \\
\text { Deviation }\end{array}$} \\
\cline { 2 - 5 } & Minimum & Maximum & Median & Mean & 0.0136 \\
\hline Freshwater $\left(29.5^{\circ} \mathrm{C}\right)$ & 0.0007 & 0.0475 & 0.003 & 0.0083 & 0.146 \\
\hline $\begin{array}{l}\text { Freshwater } \\
\left(50^{\circ} \mathrm{C} \text { to } 100^{\circ} \mathrm{C}\right)\end{array}$ & 0.037 & 0.51 & 0.229 & 0.248 & 3.346 \\
\hline Saltwater $\left(26.7^{\circ} \mathrm{C}\right)$ & 0.0014 & 14.787 & 0.7362 & 1.939 & \\
\hline
\end{tabular}

NOTE: Calculated in aqueous-316L.x/s in Appendix II from values in Table 4-3 and Table 4-5. Archived in output DTN: MO0409SPAACRWP.000. 


\subsubsection{Boil-Down Tests}

The results from 80-week boil-down tests are located in Table 4-6. These results are included as they show the trend of Stainless Steel Type 304L corrosion as the test solution is gradually concentrated by evaporation. The data on the corrosion of Stainless Steel Type 304L in a boil-down environment is located in Figure 6-6. As there are no published data on the solution chemistry of these tests, other than that the solution was slowly concentrated through evaporation, no statistical analysis was done for this set of corrosion values. However, Figure 6-6 below shows that after approximately one year, the rate of corrosion levels off at around $3 \mu \mathrm{m} / \mathrm{yr}$.

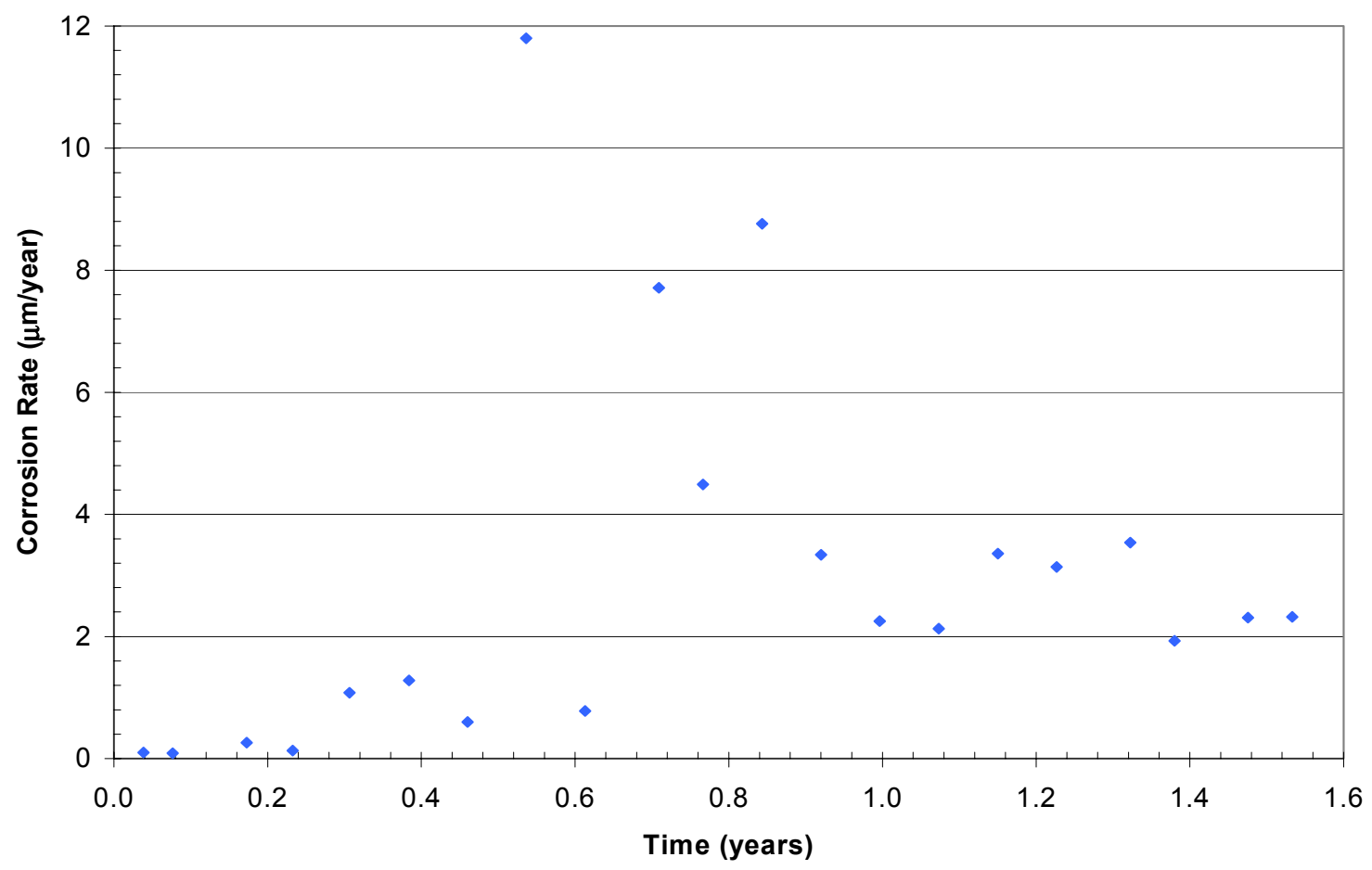

NOTE: Graphical representation of values in Table 4-6. Graphed in aqueous-304L.x/s in Appendix II. Archived in output DTN: MO0409SPAACRWP.000.

Figure 6-6. Experimental Results of Boil-Down Tests

\subsubsection{Effects of Radiolysis}

Radiolysis is defined as chemical decomposition caused by radiation. In aqueous solutions, radiolysis can produce a number of transient radicals, ions, and molecular species, including $\mathrm{H} \cdot, \cdot \mathrm{OH}, e_{\text {aq }}^{-}, \mathrm{H}_{3} \mathrm{O}^{+}, \mathrm{OH}^{-}, \mathrm{H}_{2}, \mathrm{H}_{2} \mathrm{O}_{2}, \mathrm{O}_{2}, \mathrm{O}_{2}^{-}, \mathrm{HO}_{2}$, oxides of nitrogen, and dilute nitric acid (Beavers and Durr 1991 [DIRS 159341], McCright et al. 1987 [DIRS 159336], Glass et al. 1986 [DIRS 105021]). When J-13 well water undergoes radiolysis, the oxygenated water would allow $\mathrm{O}_{2}$ and $\mathrm{H}_{2} \mathrm{O}_{2}$ to form as the dominant species in solution with smaller concentrations of $\mathrm{O}_{2}{ }^{-}$and much smaller steady state concentrations of $\mathrm{H}_{2}, \cdot \mathrm{OH}$ and $\mathrm{H} \cdot$ (Glass et al. 1986 [DIRS 105021]). Therefore, radiolysis is expected to increase the oxidizing nature of the aqueous fluid entering the waste package. From the report by Wallen and Olsson (1977 [DIRS 164948]), it is known 
that the addition of molybdenum can have a corrosive effect in highly oxidizing conditions. This indicates that if radiolysis increases the oxidizing conditions of the waste package, the corrosion rate of the Stainless Steel Type 316 series and Neutronit will increase. Beavers and Durr (1991 [DIRS 159341]) show that with increasing $\mathrm{H}_{2} \mathrm{O}_{2}$ concentration, the corrosion rate of Stainless Steel Type 304L increases (Table 4-7). The results of those experiments are shown in Figure 6-7.

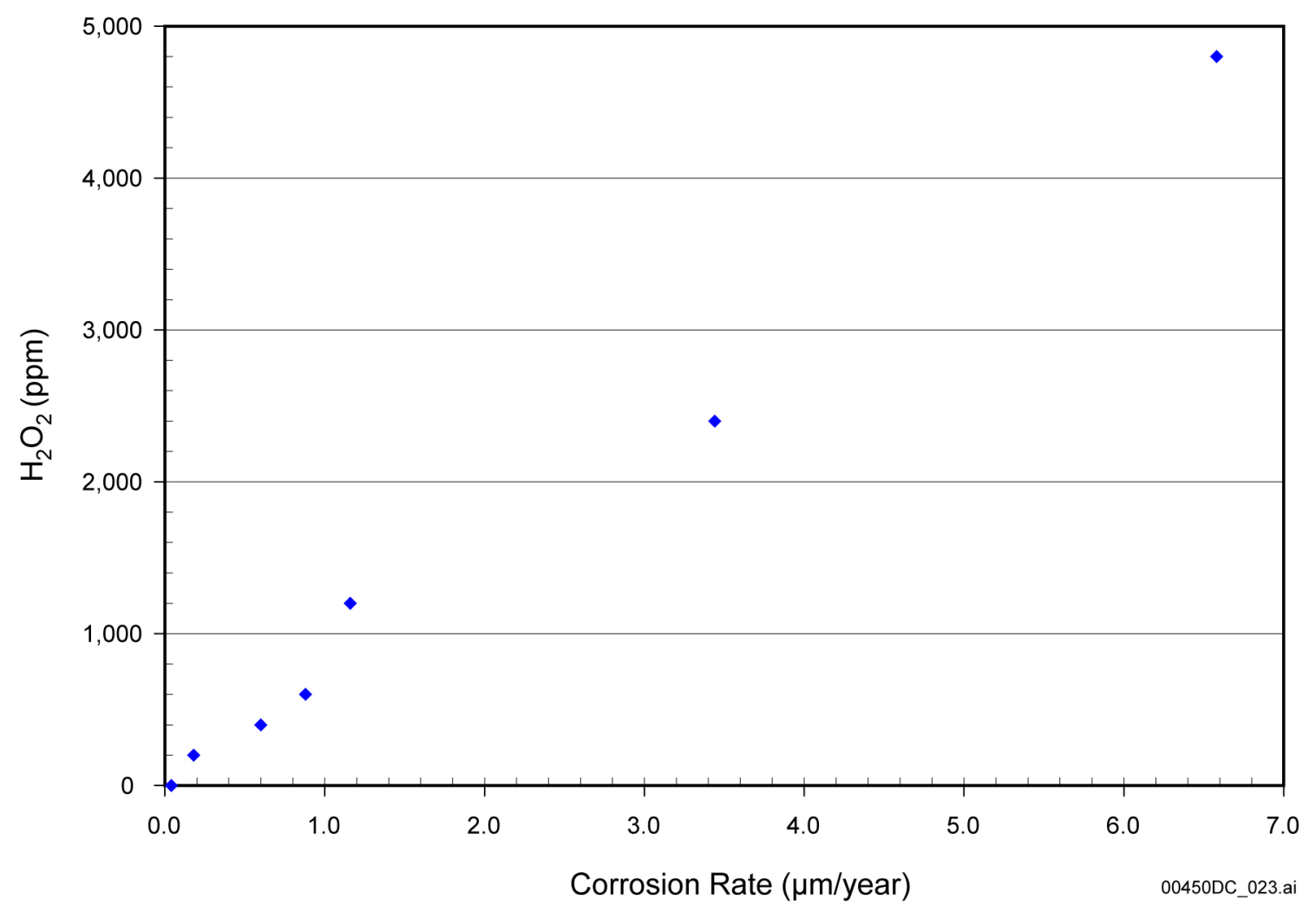

NOTE: Graphical representation of values in Table 4-7. Graphed in aqueous-304L.xls in Appendix II. Archived in output DTN: MO0409SPAACRWP.000.

Figure 6-7. Corrosion Rate of Stainless Steel Type 304L as a Function of $\mathrm{H}_{2} \mathrm{O}_{2}$ from Radiolysis

\subsubsection{Stainless Steel Sensitization}

Stainless steels are susceptible to developing a "sensitized" microstructure when exposed to high temperatures over short time periods. Sensitization causes chromium rich carbides to precipitate from solid solution in the steel, producing chromium-depleted zones around the carbides (McCright et al. 1987 [DIRS 159336]; Fox and McCright 1983 [DIRS 159344]). The protective passive film that forms on the sensitized grain boundary is, therefore, not as stable as that on the bulk of the material because of the depleted zones of chromium. This leaves the stainless steel surface open to preferential attack (localized corrosion), especially by oxidizing media.

High carbon-content steels (Stainless Steel Types 316/304) are very susceptible to sensitization. Lower carbon-content steels are preferable (Stainless Steel Types 316L/304L), as these have been shown to hold up better to sensitization than their higher carbon counterparts (Gdowski and Bullen 1988 [DIRS 100860]; Shreir et al. 1998 [DIRS 100891], pp. 3-54 through 3-57). For 
Stainless Steel Type 316 NG, with carbon content below $0.02 \%$, no sensitization has been observed (Gdowski and Bullen 1988 [DIRS 100860]). In fact, Stainless Steel Type 316NG is the material suggested to replace Stainless Steel Type 304L in BWRs, since sensitization of Stainless Steel Type 304L causes it to corrode (Fox and McCright 1983 [DIRS 159344]). Other work shows that the molybdenum in the Stainless Steel Type 316 series impedes the diffusion of carbon atoms, slowing the growth of carbide nuclei, thus making the Stainless Steel Type 316 more resistant to sensitization (McCright et al. 1987 [DIRS 159336]).

Chromium carbide precipitation usually occurs in the temperature range from $500^{\circ} \mathrm{C}$ to $850^{\circ} \mathrm{C}$ (through heat treatments) with the rate of precipitation controlled by chromium diffusion (Gdowski and Bullen 1988 [DIRS 100860]; Fox and McCright 1983 [DIRS 159344]). However, steels are rarely kept at these high temperatures for sufficient amounts of time to become sensitized. Therefore, it must be determined whether stainless steel placed in the repository environment, with temperatures ranging from $85^{\circ} \mathrm{C}$ to $280^{\circ} \mathrm{C}$, will undergo low temperature sensitization (LTS). Drift-Scale Coupled Processes (DST and THC Seepage) Models (BSC 2004 [DIRS 168848], Section 6.5.5.1) indicates that the repository temperatures will decrease over time. Briant et al. (1982 [DIRS 159345]) estimate that at $200^{\circ} \mathrm{C}$, it would take 4,000 years for Stainless Steel Type 304L to become sensitized. From these two references, it can be inferred that sensitization of the Stainless Steel Types 304 series, 316 series, and Neutronit) by early repository temperatures is unlikely. Therefore, no analysis on the corrosion of a sensitized stainless steel is presented here.

\subsubsection{Steel Containing Neutron Absorbers}

\subsubsection{Neutronit}

Kügler (1997 [DIRS 134327]) states that the corrosion resistance of Neutronit is similar to that of AISI Stainless Steel Type 321. The corrosion rates of Neutronit (based on the corrosion of Stainless Steel Type 321) can be found in Tables 4-8 and 4-9. Like other stainless steels, it displays excellent corrosion resistance, especially in dilute waters. A rate of $40 \mu \mathrm{m} / \mathrm{yr}$ has been suggested in Scoping Corrosion Tests on Candidate Waste Package Basket Materials for the Yucca Mountain Project (Van Konynenburg et al. 1998 [DIRS 100948]) after 96-hour exposure to highly corrosive media containing 0.01 -molar concentrations of radiolysis products (nitric acid and hydrogen peroxide) and chloride buffered at $\mathrm{pH}$ 4. In-Package Chemistry Abstraction (BSC 2004 [DIRS 167621], Appendix III) has shown that lower concentrations of nitric acid and hydrogen peroxide will be produced by radiolysis, having little effect on the $\mathrm{pH}$ and chemistry inside the waste package. Therefore, the test conditions used by Van Konynenburg et al. (1998 [DIRS 100948]) are not representative of expected repository conditions and their rate value was not used for this analysis. The statistical information on the corrosion of Stainless Steel Type 321 is located in Table 6-6, Figure 6-8 and Figure 6-9. 


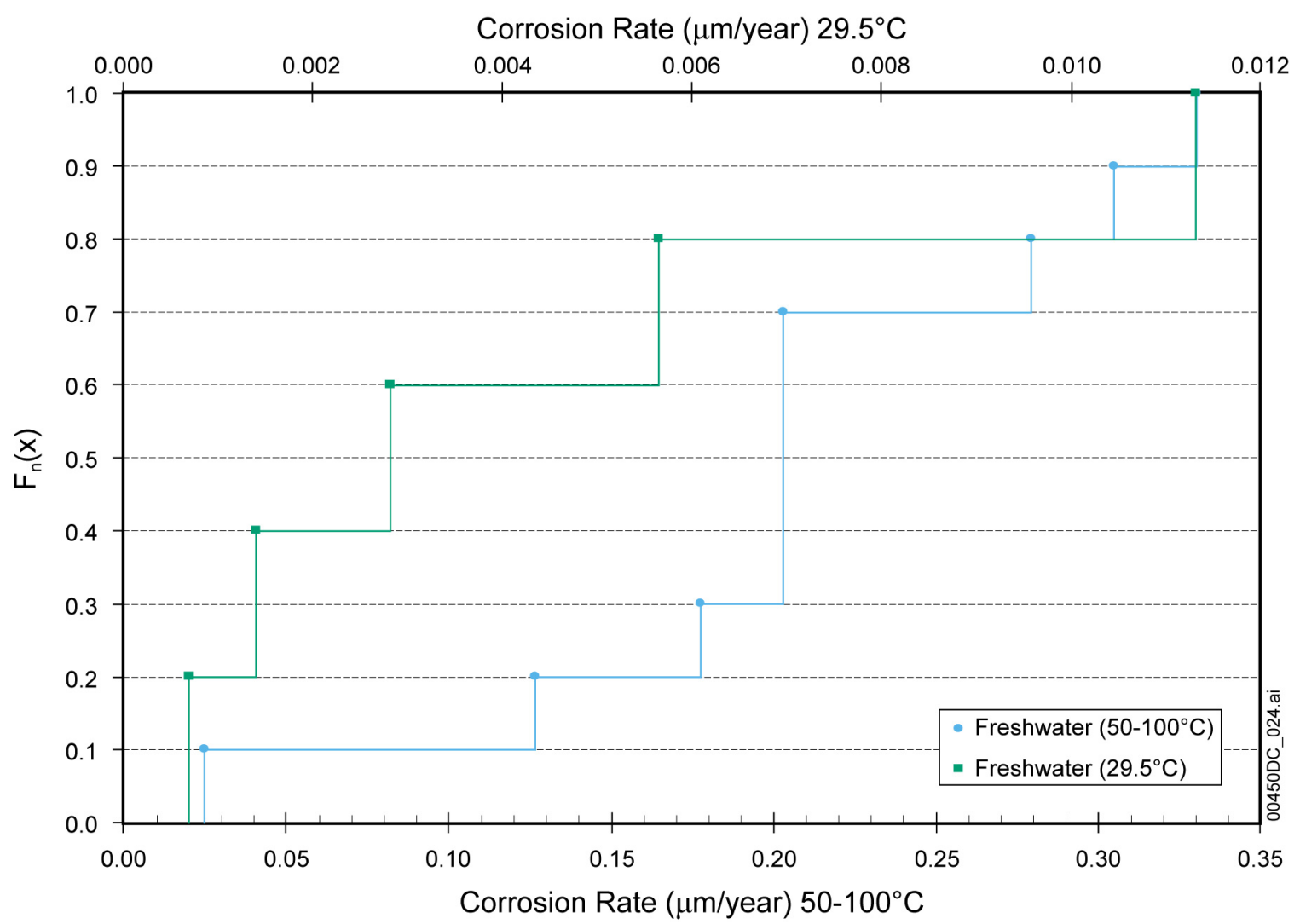

NOTE: $\quad$ ECDF calculated in ECDF_metals.x/s in Appendix II from values in Table 4-8. Archived in output DTN: MO0409SPAACRWP.000.

Figure 6-8. ECDF for Neutronit (Using Stainless Steel Type 321 Surrogate) in Freshwater

Table 6-6. Minimum, Maximum, Mean, and Median Aqueous Corrosion Rates of Neutronit (Using Stainless Steel Type 321 Surrogate)

\begin{tabular}{|l|c|c|c|c|c|}
\hline \multirow{2}{*}{ Environment } & \multicolumn{4}{|c|}{ Corrosion Rate $(\mu \mathrm{m} / \mathrm{yr})$} & $\begin{array}{c}\text { Standard } \\
\text { Deviation }\end{array}$ \\
\cline { 2 - 6 } & Minimum & Maximum & Median & Mean & 0.004 \\
\hline Freshwater $\left(29.5^{\circ} \mathrm{C}\right)$ & 0.001 & 0.011 & 0.003 & 0.004 & 0.088 \\
\hline $\begin{array}{l}\text { Freshwater }\left(50^{\circ} \mathrm{C} \text { to }\right. \\
\left.100^{\circ} \mathrm{C}\right)\end{array}$ & 0.025 & 0.330 & 0.203 & 0.206 & 10.19 \\
\hline Saltwater $\left(26.7^{\circ} \mathrm{C}\right)$ & 1.81 & 29.22 & 7.38 & 11.06 & \\
\hline
\end{tabular}

NOTE: Calculated in aqueous-B-Gd-steels.x/s in Appendix II from values in Table 4-8 and Table 4-9. Archived in output DTN: MO0409SPAACRWP.000. 


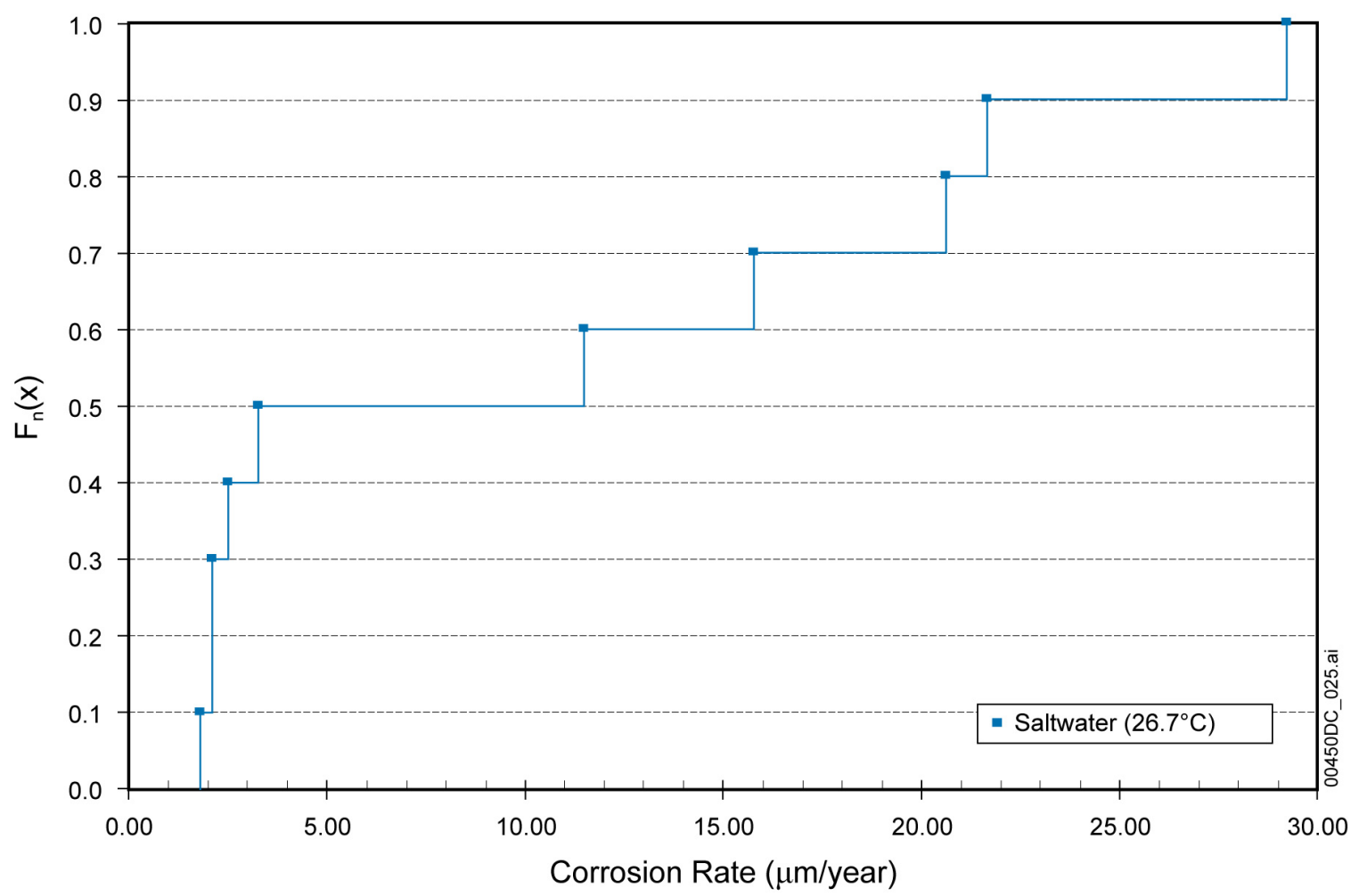

NOTE: $\quad$ ECDF calculated in ECDF_metals.xls in Appendix II from values in Table 4-9. Archived in output DTN: MO0409SPAACRWP.000.

Figure 6-9. ECDF for Neutronit (Using Stainless Steel Type 321 Surrogate) in Saltwater

\subsubsection{Borated Stainless Steel Type 304L}

The effects of boron content have also been studied for Stainless Steel Type 304. Most corrosion tests for this material have been conducted in extremely hostile environments such as boiling nitric acid, sulfuric acid and 7N-HNO 3 (Smith et al. 1992 [DIRS 103441]; EPRI 1986 [DIRS 159367]; and Butler 1963 [DIRS 159368]).

Cole (1976 [DIRS 159369]) presents data on corrosion of borated Stainless Steel Type 304 in environments, which may be encountered in the waste package, and had the following conclusions:

- Stainless steel alloyed with 1.5 percent boron degraded faster than steel containing only 0.3 percent boron showing that increasing boron content increases degradation rate

- Corrosion at $50^{\circ} \mathrm{C}$ in concentrated solutions was much greater than at ambient temperatures in dilute solutions.

Other conclusions on the effects of zirconium and aluminum on the corrosion rates included:

- Corrosion decreased as aluminum complexing of the solution increased, and

- Zirconium serves as a sacrificial metal to reduce the corrosion of borated Stainless Steel Type 304 with 1.5 percent boron. 
The statistical information on the corrosion of Stainless Steel Type 304L is located in Table 6-7, Figure 6-10, and Figure 6-11. For this analysis, the values for 1.5 percent boron at $100^{\circ} \mathrm{C}$ corresponding to 6.04 and 6.44 mils per month (Table 4-11) as well as 6.14 and 5.99 mils per month (Table 4-11) for ambient temperatures were not used $(1 \mathrm{mil}=25.4 \mu \mathrm{m})$. Because these rates are so much higher than the other rates shown for similar conditions, it is likely that these values represent localized corrosion data and are therefore inappropriate for use in the analysis.

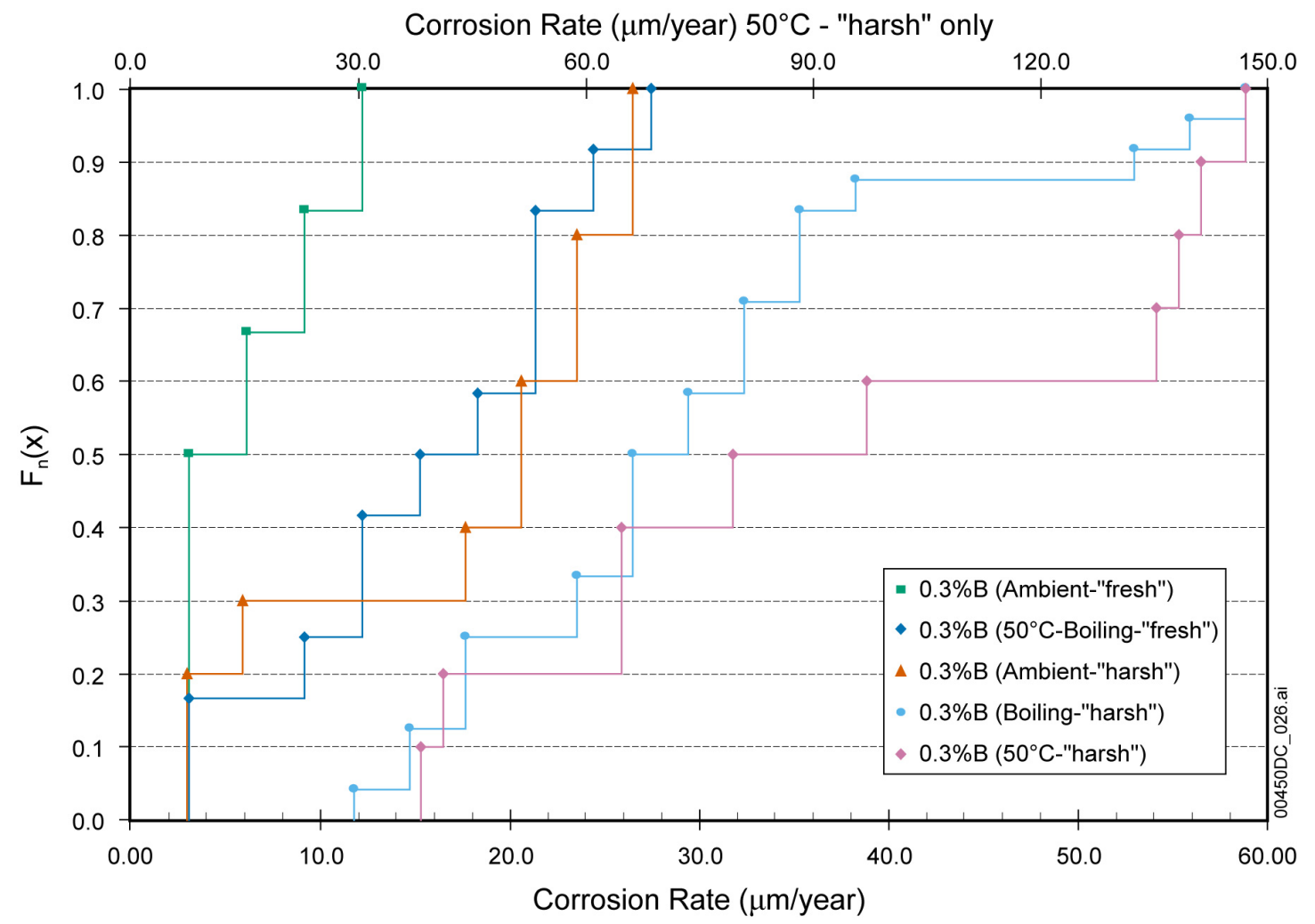

NOTE: $\quad$ ECDF calculated in ECDF_metals.x/s in Appendix II from values in Table 4-10 and Table 4-11. Archived in output DTN: MO0409SPAACRWP.000.

Figure 6-10. ECDF for 0.3 Percent Borated Stainless Steel Type 304 in All Water Types Investigated 


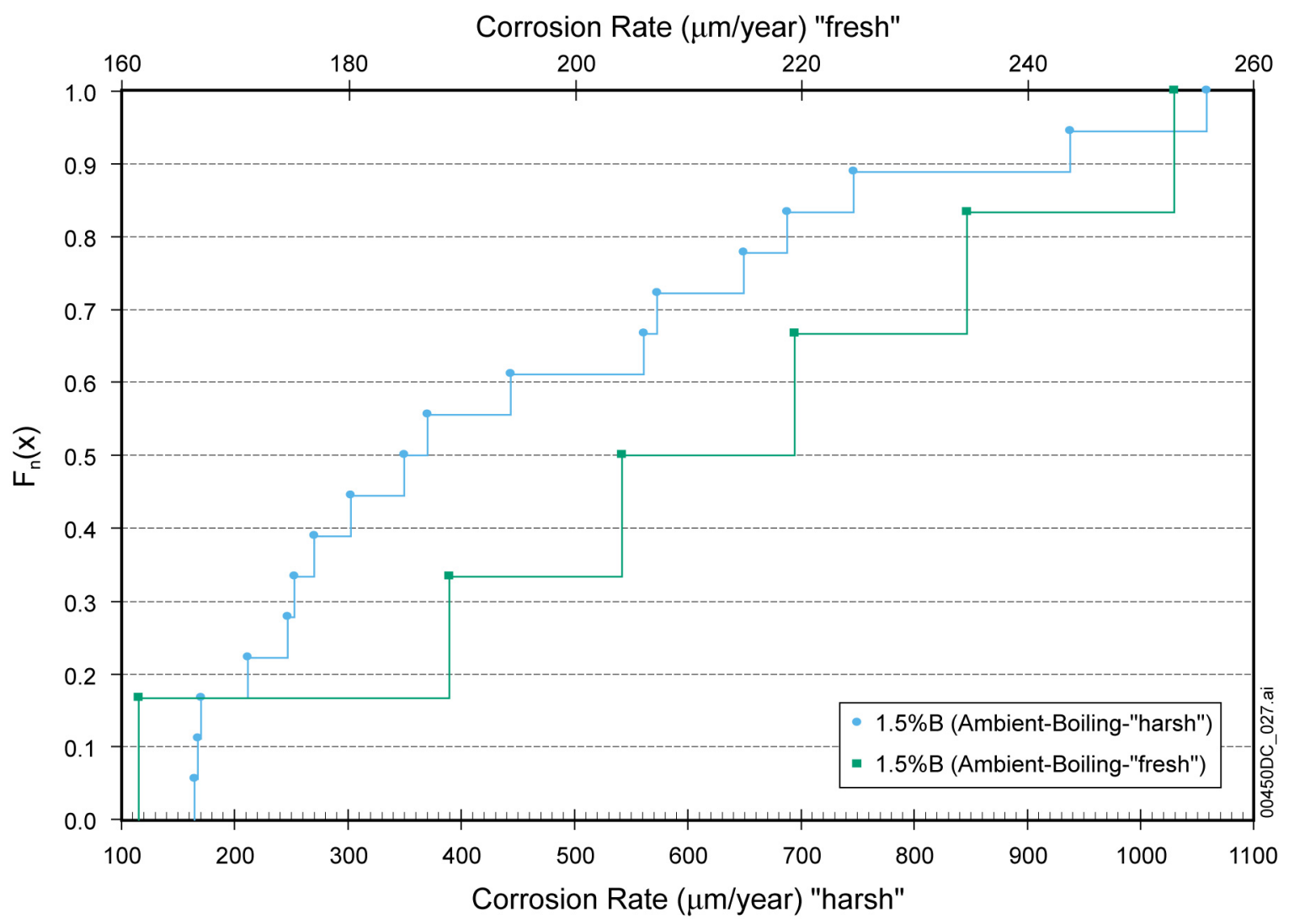

NOTE: $\quad$ ECDF calculated in ECDF_metals.xls in Appendix II from values in Table 4-10 and Table 4-11. Archived in output DTN: MO0409SPAACRWP.000.

Figure 6-11. ECDF for 1.5 Percent Borated Stainless Steel Type 304 in All Water Types Investigated

Table 6-7. Minimum, Maximum, Mean, and Median Aqueous Corrosion Rates of Borated Stainless Steel Type 304

\begin{tabular}{|c|c|c|c|c|c|}
\hline \multirow{2}{*}{ Environment } & \multicolumn{4}{|c|}{ Corrosion Rate $(\mu \mathrm{m} / \mathrm{yr})$} & \multirow{2}{*}{$\begin{array}{l}\text { Standard } \\
\text { Deviation }\end{array}$} \\
\hline & Minimum & Maximum & Median & Mean & \\
\hline $\begin{array}{l}0.3 \% \mathrm{~B} \text { (Ambient- } \\
\text { "Fresh") }\end{array}$ & 3.05 & 12.19 & 4.57 & 6.10 & 3.86 \\
\hline $\begin{array}{l}0.3 \% \mathrm{~B}\left(50^{\circ} \mathrm{C}-\text { Boiling- }\right. \\
\text { "Fresh") }\end{array}$ & 3.05 & 27.43 & 16.76 & 15.75 & 7.99 \\
\hline $\begin{array}{l}1.5 \% B \text { (Ambient- } \\
\text { Boiling-"Fresh") }\end{array}$ & 161.54 & 252.98 & 211.84 & 210.31 & 32.77 \\
\hline $\begin{array}{l}0.3 \% B \text { (Ambient- } \\
\text { "Harsh") }\end{array}$ & 2.94 & 26.46 & 20.58 & 17.05 & 9.48 \\
\hline $\begin{array}{l}0.3 \% \mathrm{~B}\left(50^{\circ} \mathrm{C}-\right. \\
\text { "Harsh") }\end{array}$ & 38.22 & 147.00 & 88.20 & 94.69 & 42.85 \\
\hline $\begin{array}{l}0.3 \% B \text { (Boiling- } \\
\text { "Harsh") }\end{array}$ & 11.76 & 58.80 & 27.93 & 29.77 & 12.48 \\
\hline $\begin{array}{l}1.5 \% B \text { (Ambient- } \\
\text { Boiling-"Harsh") }\end{array}$ & 164.64 & $1,058.40$ & 360.15 & 453.74 & 272.96 \\
\hline
\end{tabular}

NOTE: Calculated in aqueous-B-Gd-steels.x/s in Appendix II from values in Table 4-10 and Table 4-11. Archived in output DTN: MO0409SPAACRWP.000. 


\subsubsection{Gadolinium-Alloyed Stainless Steel Type 304L}

Cole (1976 [DIRS 159369]) presents data on corrosion of a gadolinium-alloyed Stainless Steel Type 304 in environments that may be encountered in the waste package. The statistical information on the corrosion of gadolinium-alloyed Stainless Steel Type 304L is located in Table 6-8.

Table 6-8. Comparison of Minimum, Maximum, Mean, and Median Corrosion Rates of GadoliniumAlloyed and Non Gadolinium-Alloyed Stainless Steel Type 304L

\begin{tabular}{|c|c|c|c|}
\hline \multirow[b]{2}{*}{ Environment } & \multicolumn{3}{|c|}{ Corrosion Rate $(\mu \mathrm{m} / \mathrm{yr})$} \\
\hline & Minimum & Maximum & Mean \\
\hline $0.3 \%$ Gd 304 (Ambient-Boiling-"Fresh") & 3.05 & 15.24 & 9.14 \\
\hline $304 \mathrm{~L}$ Freshwater $\left(25^{\circ} \mathrm{C} \text { to } 100^{\circ} \mathrm{C}\right)^{\mathrm{b}}$ & 0.001 & 1.57 & 0.214 \\
\hline Increase in Corrosion & 3,050 times & 9.71 times & 33.27 times \\
\hline $0.3 \%$ Gd 304 (Ambient-"Harsh") & 2.94 & 2.94 & 2.94 \\
\hline 304L Saltwater $\left(<27^{\circ} \mathrm{C}\right)^{\mathrm{b}}$ & 1.588 & 39.147 & 11.44 \\
\hline Increase in Corrosion & 1.85 times & 0.08 times & 0.26 times \\
\hline $0.3 \%$ Gd 304 (Boiling-"Harsh") & 5.88 & 23.52 & 11.03 \\
\hline 304L Saltwater $\left(90^{\circ} \mathrm{C}\right)^{\mathrm{b}}$ & 0.66 & 15.9 & 5.816 \\
\hline Increase in Corrosion & 8.91 times & 1.48 times & 1.90 times \\
\hline
\end{tabular}

NOTES: ${ }^{a}$ Values calculated in aqueous-B-Gd-steels.xls in Appendix II from values in Table 4-12; archived in output DTN: MO0409SPAACRWP.000.

balues from Table 6-4.

\subsubsection{Carbon Steel Type A516}

As pointed out in the sections above, the extent to which corrosion proceeds is highly dependent on the elemental composition of the material. Carbon steel does not have the protective alloying effects from the addition of chromium, nickel, and molybdenum. Therefore, its rates of corrosion tend to be much higher than those of stainless steel. The corrosion rates of Carbon Steel Type A516 can be found in aqueous-A516.xls in Appendix II and Tables 4-13 through 4-20.

Unlike stainless steel, the corrosion rate of carbon steel is highly dependent on the temperature of the system. From the experimental data of McCright (1998 [DIRS 114637]) presented in Table 4-13 through Table 4-20, it is shown that corrosion at $60^{\circ} \mathrm{C}$ is more aggressive than corrosion at $90^{\circ} \mathrm{C}$. This is corroborated by Brasher and Mercer (1968 [DIRS 100883]), whose data on the corrosion of mild/carbon steel for different temperatures is shown in Figure 6-12. The highest corrosion rate occurs at $60^{\circ} \mathrm{C}$. From this temperature $\left(60^{\circ} \mathrm{C}\right)$, the corrosion rate decreases when the temperature either increases or decreases (i.e., corrosion rates for both $25^{\circ} \mathrm{C}$ and $90^{\circ} \mathrm{C}$ will be lower than the $60^{\circ} \mathrm{C}$ rates). As can be seen from Figure 6-12, the rates for the mild steel are within the same range as those for the Carbon Steel Type A516 from Corrosion Data and Modeling, Update for Viability Assessment (McCright 1998 [DIRS 114637]). Although no specific data have been located for corrosion of Carbon Steel Type A516 at $25^{\circ} \mathrm{C}$, 
Figure 6-12 shows that the rates for mild steel at $25^{\circ} \mathrm{C}$ are slightly lower than mild steel at $90^{\circ} \mathrm{C}$. Therefore, in the absence of $25^{\circ} \mathrm{C}$ data for Carbon Steel Type A516, those presented for mild steel at $90^{\circ} \mathrm{C}$ can be used as a surrogate.

In addition, unlike stainless steel, carbon steel corrosion decreases with increasing salinity of the corroding medium. Tables 4-13 through 4-20 in Section 4.1.3.3 show that the corrosion rates for water concentrated to $1,000 \times$ that of $\mathrm{J}-13$ well water $(\mathrm{SCW})$ are significantly lower than those at the $10 \times$ concentration (SDW). These values are for laboratory conditions and not a natural setting. In the case of carbon steel, this has a great effect on the corrosion rates. In natural saline water (i.e., ocean or tidal), many other factors can increase corrosion rates, which may not be seen in waste package corrosion. These effects include water flow rates, biofouling, and pollution. With stainless steels, there was little difference between the corrosion rates in natural and laboratory settings. However, for carbon steel, the results of laboratory experiments may more closely represent the actual rates since they do not include the three factors mentioned above.

In the experiments conducted by McCright (1998 [DIRS 114637]), the corrosion rate decreases from the 0.5-year to the 1-year time period. The same behavior is shown in Figure 1 of "Carbon Steel-Corrosion by Seawater" (Matsushima 2000 [DIRS 164942]) in which the rates decrease significantly in the first 10 to 20 years. As with stainless steel, this is caused by the formation of an oxide film at the surface of the metal. However, because of the absence of chromium, nickel, and molybdenum, these films are composed of iron oxides. While intact, these films can effectively slow the corrosion of the metal, but are not as efficient as the thin hydrous chromium, silicon, and molybdenum enriched oxides that protect stainless steels. Therefore, even though a protective film forms, it cannot completely stop corrosion on the surface of the steel (Matsushima 2000 [DIRS 164944]).

Other factors affecting the corrosion of carbon steel in freshwaters include $\mathrm{pH}, \mathrm{Cl}^{-}$, bicarbonate $\left(\mathrm{HCO}_{3}{ }^{-}\right.$), and $\mathrm{SO}_{4}{ }^{2-}$ (Matsushima 2000 [DIRS 164944]). It is generally considered that over the $\mathrm{pH}$ range of approximately 4 to 10 , the corrosion rate of carbon steel is independent of the $\mathrm{pH}$ (Matsushima 2000 [DIRS 164944]). However, this changes when high concentrations of $\mathrm{Cl}^{-}$and $\mathrm{SO}_{4}{ }^{2-}$ are present in solution. The data from Corrosion Data and Modeling, Update for Viability Assessment (McCright 1998 [DIRS 114637]) shows that even in solutions with high levels of these two anions, the corrosion rates were still lower in concentrated solutions than in dilute solutions. Matsushima (2000 [DIRS 164944]) points out that as long as dissolved oxygen is controlling corrosion, the $\mathrm{Cl}^{-}$and $\mathrm{SO}_{4}{ }^{2-}$ ions will essentially have no effect on the overall general corrosion rate of steel because they do not effect the solubility of oxygen. However, they may cause a breakdown of the passive oxide film and accelerate localized corrosion. This effect is even more pronounced at lower $\mathrm{pH}$ values as shown by Satyanarayana (1981 [DIRS 159371], Figure 8). On the other hand, $\mathrm{HCO}_{3}{ }^{-}$may counteract this effect. In the presence of $\mathrm{Ca}^{2+}$, a protective $\mathrm{CaCO}_{3}$ film forms. In the absence of $\mathrm{Ca}^{2+}, \mathrm{FeCO}_{3}$ can form. Though not as protective as minerals such as $\mathrm{Fe}(\mathrm{OH})_{2}$, this layer will still have a passivating effect on the surface of the steel. 


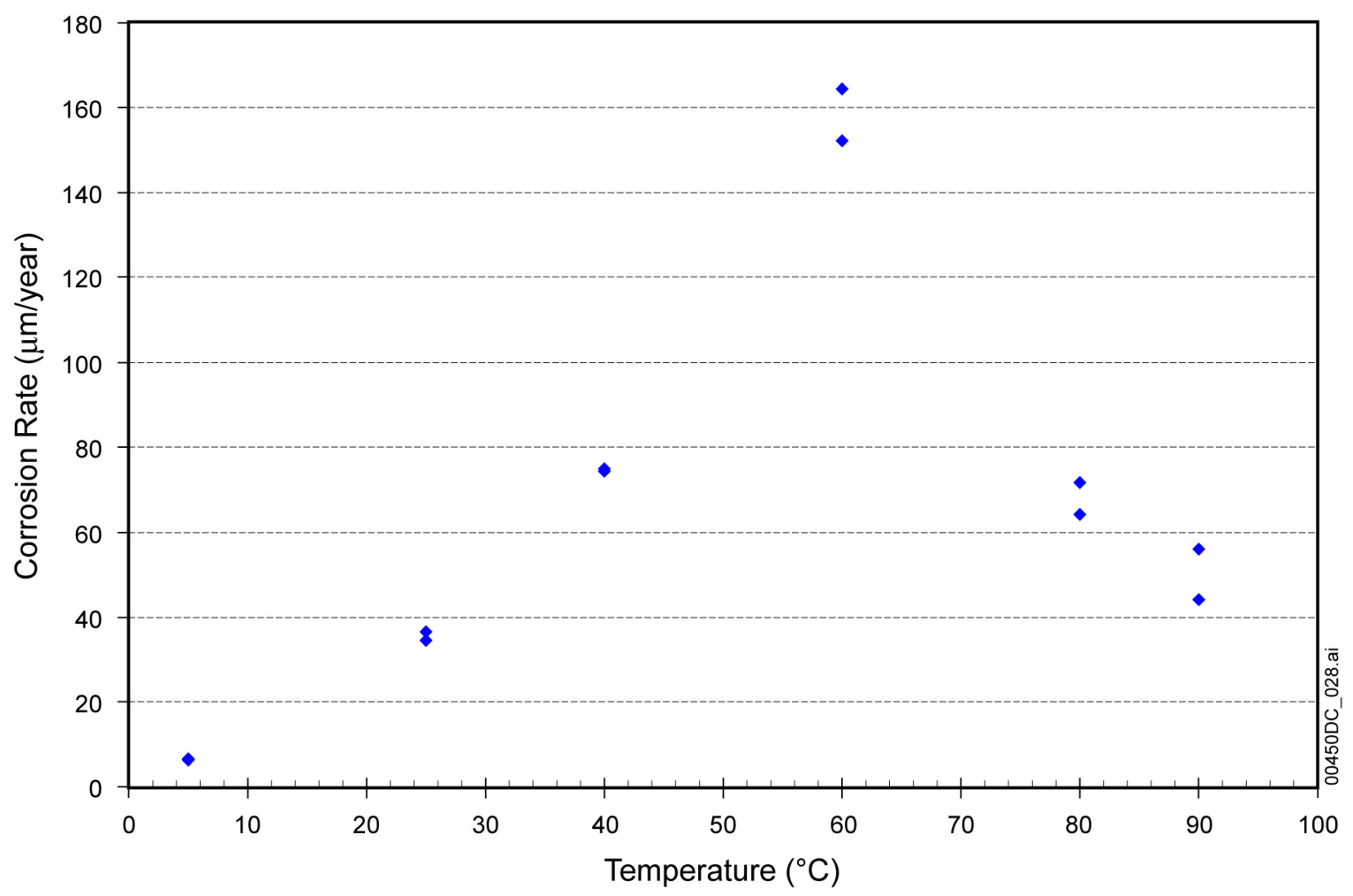

NOTE: Graphical representation of values from Brasher and Mercer 1968 [DIRS 100883]. Graphed in aqueousA516.xls in Appendix II. Archived in DTN: MO0409SPAACRWP.000.

Figure 6-12. Corrosion Rates versus Temperature for Mild Steel

The rates used to describe the corrosion of Carbon Steel Type A516 are found in Tables 4-13 through 4-20. Since carbon and low alloy steels are used extensively by many industries, a large amount of corrosion data is available in the literature. For this analysis, the data from McCright (1998 [DIRS 114637]) for Carbon Steel Types A516 and A27 was used since this study was conducted using environmental conditions expected to be present in the repository. Since Carbon Steel Type A27 is low-alloy carbon steel similar to Carbon Steel Type A516, and their corrosion rates are, therefore, similar, Carbon Steel Type A27 was also used to describe the corrosion of Carbon Steel Type A516. The statistical information on the corrosion of Carbon Steel Type A516 is located in Table 6-9 and Figure 6-13. 


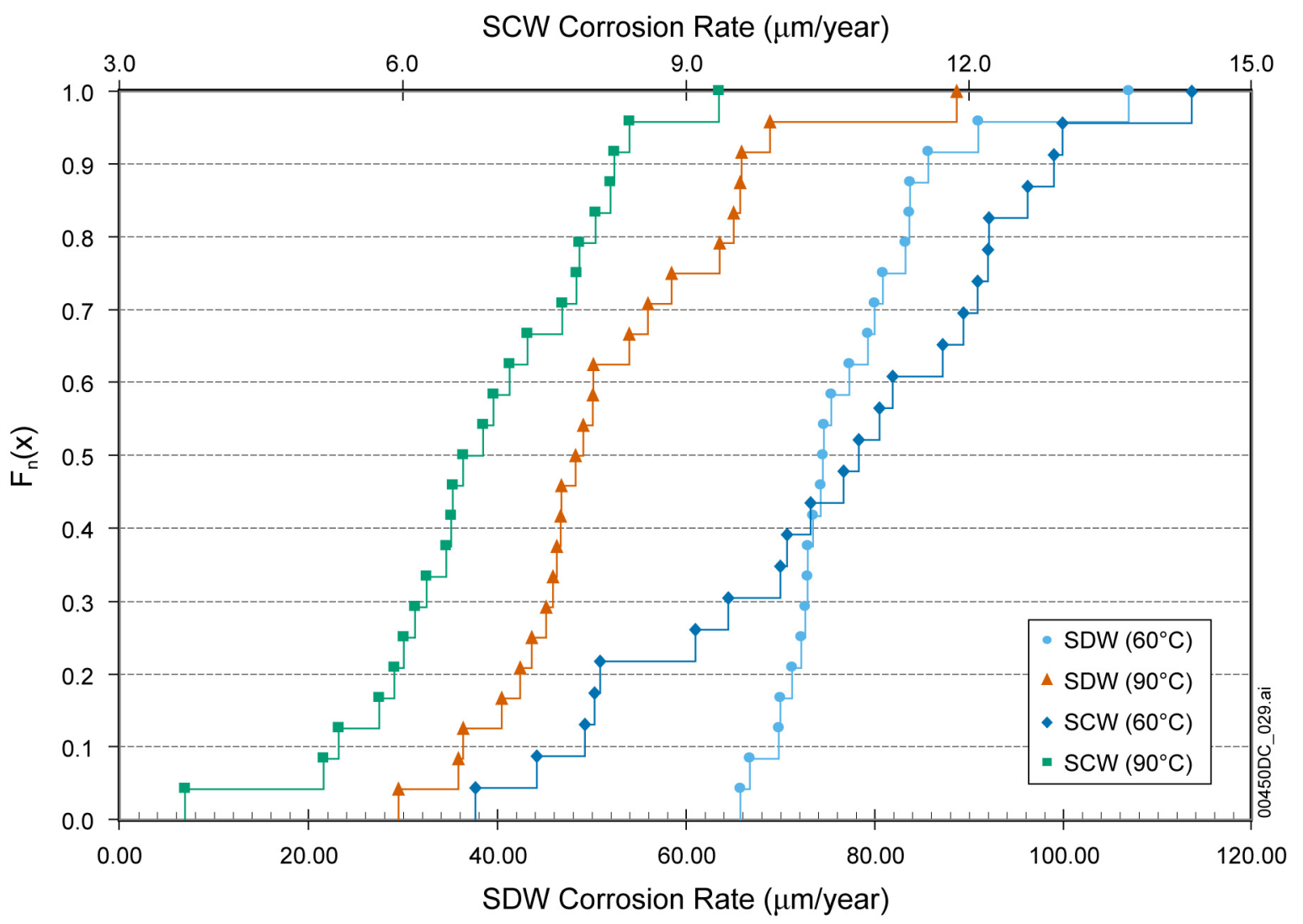

NOTE: $\quad$ ECDF calculated in ECDF_metals.x/s in Appendix II from values in Table 4-13 through Table 4-16. Archived in output DTN: MO0409SPAACRWP.000.

Figure 6-13. ECDF for Carbon Steel Type A516 in All Water Types Investigated (Time $\geq 1$ Year)

Table 6-9. Minimum, Maximum, Mean, and Median Aqueous Corrosion Rates of Carbon Steel Type A516

\begin{tabular}{|c|c|c|c|c|c|}
\hline \multirow{2}{*}{ Environment } & \multicolumn{4}{|c|}{ Corrosion Rate $(\mu \mathrm{m} / \mathrm{yr})$} & \multirow{2}{*}{$\begin{array}{l}\text { Standard } \\
\text { Deviation }\end{array}$} \\
\hline & Minimum & Maximum & Median & Mean & \\
\hline \multicolumn{6}{|c|}{ Short term ( $\leq 0.53$ years) } \\
\hline $\operatorname{SDW}\left(60^{\circ} \mathrm{C}\right)$ & 78.71 & 130.70 & 101.95 & 102.71 & 12.37 \\
\hline $\operatorname{SDW}\left(90^{\circ} \mathrm{C}\right)$ & 58.08 & 130.02 & 77.05 & 81.14 & 15.13 \\
\hline $\operatorname{SCW}\left(60^{\circ} \mathrm{C}\right)$ & 50.25 & 104.20 & 62.77 & 68.08 & 14.17 \\
\hline $\operatorname{SCW}\left(90^{\circ} \mathrm{C}\right)$ & 7.39 & 22.06 & 12.42 & 12.78 & 3.77 \\
\hline \multicolumn{6}{|c|}{ Long term ( $\geq 1$ year) } \\
\hline $\operatorname{SDW}\left(60^{\circ} \mathrm{C}\right)$ & 65.77 & 106.93 & 74.56 & 77.43 & 8.83 \\
\hline $\operatorname{SDW}\left(90^{\circ} \mathrm{C}\right)$ & 29.53 & 88.68 & 48.70 & 51.80 & 12.99 \\
\hline $\operatorname{SCW}\left(60^{\circ} \mathrm{C}\right)$ & 6.77 & 14.36 & 10.83 & 10.61 & 2.02 \\
\hline $\operatorname{SCW}\left(90^{\circ} \mathrm{C}\right)$ & 3.69 & 9.35 & 6.75 & 6.84 & 1.25 \\
\hline
\end{tabular}

NOTE: Calculated in aqueous-A516.x/s in Appendix II from values in Tables 4-13 through 4-20. Archived in output DTN: MO0409SPAACRWP.000. 


\subsubsection{Aluminum Alloys}

A brief synopsis of aluminum corrosion (Hollingsworth and Hunsicker 1987 [DIRS 150403]; Ghali 2000 [DIRS 164946]) is presented below.

Aluminum Alloy Type 1100 ("commercially pure aluminum") contains 99.0 to 99.3 percent aluminum and has a higher resistance to corrosion in most acid and neutral solutions than lower purity aluminum or aluminum alloys. The nonheat-treatable alloys (1 $\mathrm{xxx}, 3 \mathrm{xxx}, 4 \mathrm{xxx}$, and $5 \mathrm{xxx}$ series), as well as the heat-treatable alloys of the group $6 \mathrm{xxx}$ and noncopper containing $7 \mathrm{xxx}$ alloys are highly resistant to general corrosion, with the $6 \mathrm{xxx}$ and $7 \mathrm{xxx}$ being the least resistant. Alloys of the $2 \mathrm{xxx}$ and copper containing $7 \mathrm{xxx}$ series generally have lower resistance to general corrosion. Aluminum is a surface active metal and its resistance to corrosion thus depends highly on the formation of a protective oxide film. Aluminum passivity develops in the $\mathrm{pH}$ range of about 5 to 8.5 . This passivity depends greatly on the temperature, the form of the oxide, $\mathrm{O}_{2}$ content, $\mathrm{pH}$, and the aqueous medium.

At low temperatures, aqueous solutions are usually less corrosive than at high temperatures. However, for some solutions, increasing temperatures (usually above $80^{\circ} \mathrm{C}$ ) results in a decrease in aluminum corrosion. The protective oxide film at ambient temperatures is only a few nanometers thick and usually amorphous or predominantly bayerite $\left(\mathrm{Al}(\mathrm{OH})_{3}\right)$. At high temperatures, thicker films may form. Highly protective crystalline films of boehmite (AlOOH) may form in water near the boiling point. The formation of crystalline films is more likely in alkaline solutions. During aging of aluminum hydroxides, gibbsite $\left(\mathrm{Al}(\mathrm{OH})_{3}\right)$ may also form. A protective film does not form above $230^{\circ} \mathrm{C}$.

Oxygen also affects the rate of corrosion for aluminum. In deaerated solutions, the corrosion of aluminum tends to be very slow, whereas in the presence of oxygen, corrosion is accelerated. This is even more pronounced in acidic solutions. Other dissolved gases such as carbon dioxide and hydrogen sulfide appear to have slight inhibiting action on the corrosion of aluminum by aqueous mediums whereas hydrogen chloride is very corrosive to aluminum.

As mentioned above, aluminum and its alloys tend to be passivated between $\mathrm{pH} 5$ and 8.5. It corrodes under both acidic and alkaline conditions to produce $\mathrm{Al}^{3+}$ and $\mathrm{AlO}_{2}{ }^{-}$(aluminate) ions respectively. There is no general relationship between $\mathrm{pH}$ and corrosion rate because other ions present in solution largely influence corrosion rates. For example, concentrated solutions of nitric or acetic acid may not effect the corrosion rate while dilute solutions of nitric, sulfuric, and hydrochloric acid solutions are very corrosive. Likewise, alkaline solutions may not attack aluminum in the presence of silicates, but in the absence of silicates, corrosion rates may be very high.

In distilled water, even at high temperatures, aluminum corrosion is quite low. This is also true of clean natural waters. However, some waters can cause severe localized corrosion such as pitting. In natural freshwaters, the important factors for corrosion have been presented above. For seawater, corrosion is primarily through pitting. Rates of pitting usually range from 3 to $6 \mu \mathrm{m} / \mathrm{yr}$ during the first year. An average over a 10-year period shows the rate of pitting to be 0.8 to $1.5 \mu \mathrm{m} / \mathrm{yr}$. The decreasing rates over time indicate the tendency of older pits to become inactive. This is corroborated by Table 6-10, which shows the occurrence of pits on aluminum 
when exposed to tap water from Kingston, Ontario. Pitting tends to be the main corrosion behavior of aluminum. Pitting weight-loss data for aluminum are converted to general rates for use in EQ6 for this analysis.

Table 6-10. Pitting in Aluminum Alloys

\begin{tabular}{|c|c|c|c|c|c|c|}
\hline & \multicolumn{5}{|c|}{ Number of Pits within the Pit Depth Ranges in Time Period } \\
\hline $\begin{array}{c}\text { Pit Depth Range } \\
\text { (microns) }\end{array}$ & $\mathbf{2}$ weeks & $\mathbf{1}$ Month & 2 Months & 4 Months & 6 Months & 1 year \\
\hline 0 to 99 & 176 & 216 & 155 & 171 & 111 & 140 \\
\hline 100 to 199 & 54 & 49 & 22 & 24 & 27 & 11 \\
\hline 200 to 299 & 49 & 73 & 19 & 24 & 26 & 17 \\
\hline 300 to 399 & 21 & 98 & 48 & 37 & 41 & 18 \\
\hline 400 to 499 & 14 & 71 & 56 & 65 & 52 & 47 \\
\hline 500 to 599 & 1 & 68 & 51 & 60 & 50 & 48 \\
\hline 600 to 699 & 0 & 30 & 38 & 33 & 37 & 36 \\
\hline 700 to 799 & 1 & 16 & 32 & 43 & 43 & 22 \\
\hline 800 to 899 & - & 4 & 4 & 15 & 3 & 7 \\
\hline 900 to 999 & - & 1 & - & 3 & 3 & 1 \\
\hline 1,000 to 1,099 & - & - & - & - & & 1 \\
\hline
\end{tabular}

Source: Aziz 1956 [DIRS 159379].

NOTE: Kingston, Ontario tap water (no other data on this water was available).

Since aluminum alloys are used in many industries, saltwater corrosion data for many different types can be found in the literature. For this analysis, it was decided to use the data from one large study since using a full bibliography of values is unreasonable. The chosen values come from Hollingsworth and Hunsicker (1987 [DIRS 150403]). The corrosion rates for aluminum alloys used in this analysis for fresh and seawater are located in Section 4.1.3.4 and in aluminum_.xls on Appendix II. The statistical information on the corrosion of aluminum alloys is located in Table 6-11 and Figure 6-14. More detail of the ECDF is presented in Figure 6-15 through Figure 6-17. 


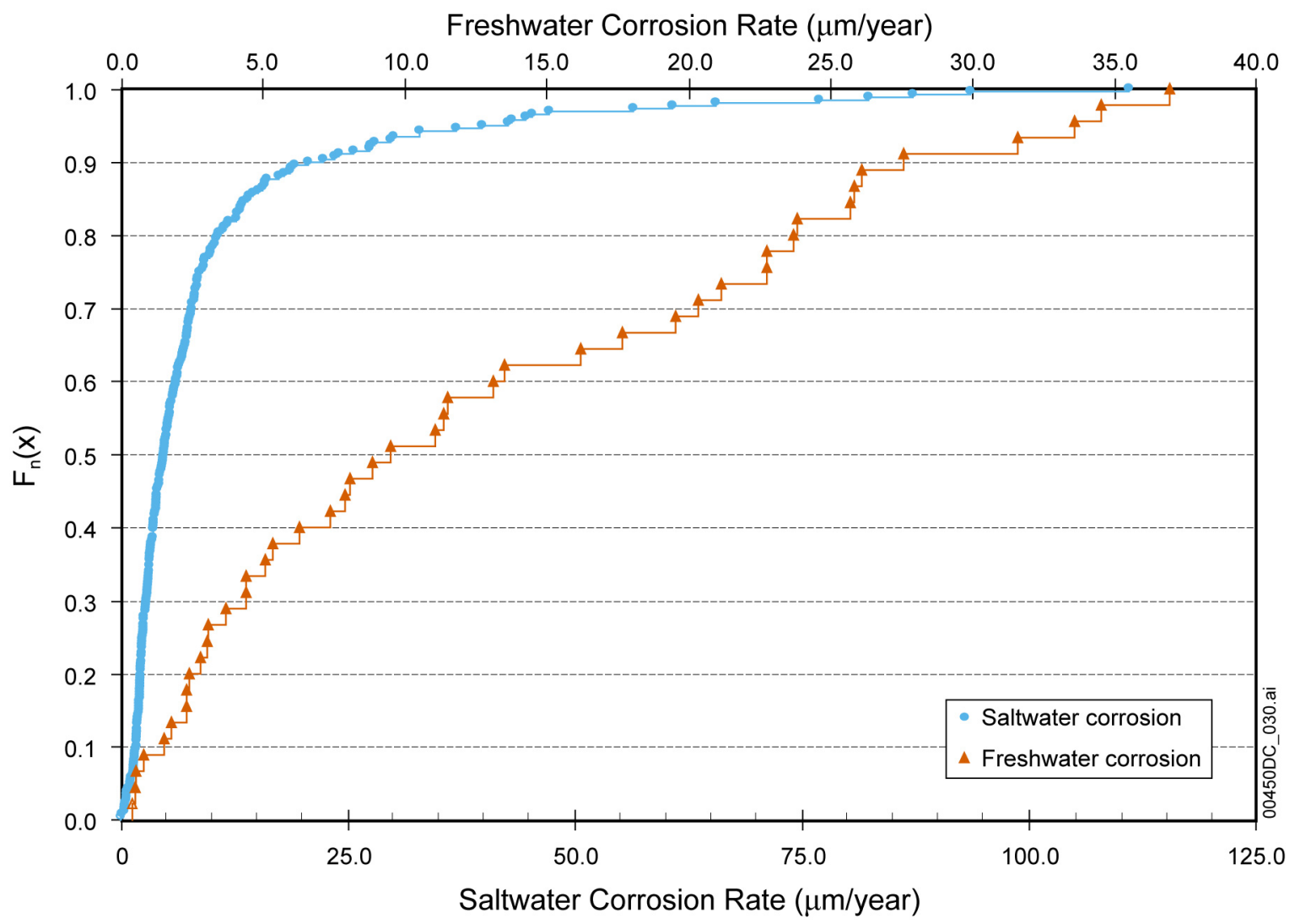

NOTE: ECDF calculated in ECDF_metals.xls in Appendix II from values in Table 4-21 and Table 4-22. Archived in output DTN: MO0409SPAACRWP.000.

Figure 6-14. ECDF for Aluminum Alloy 


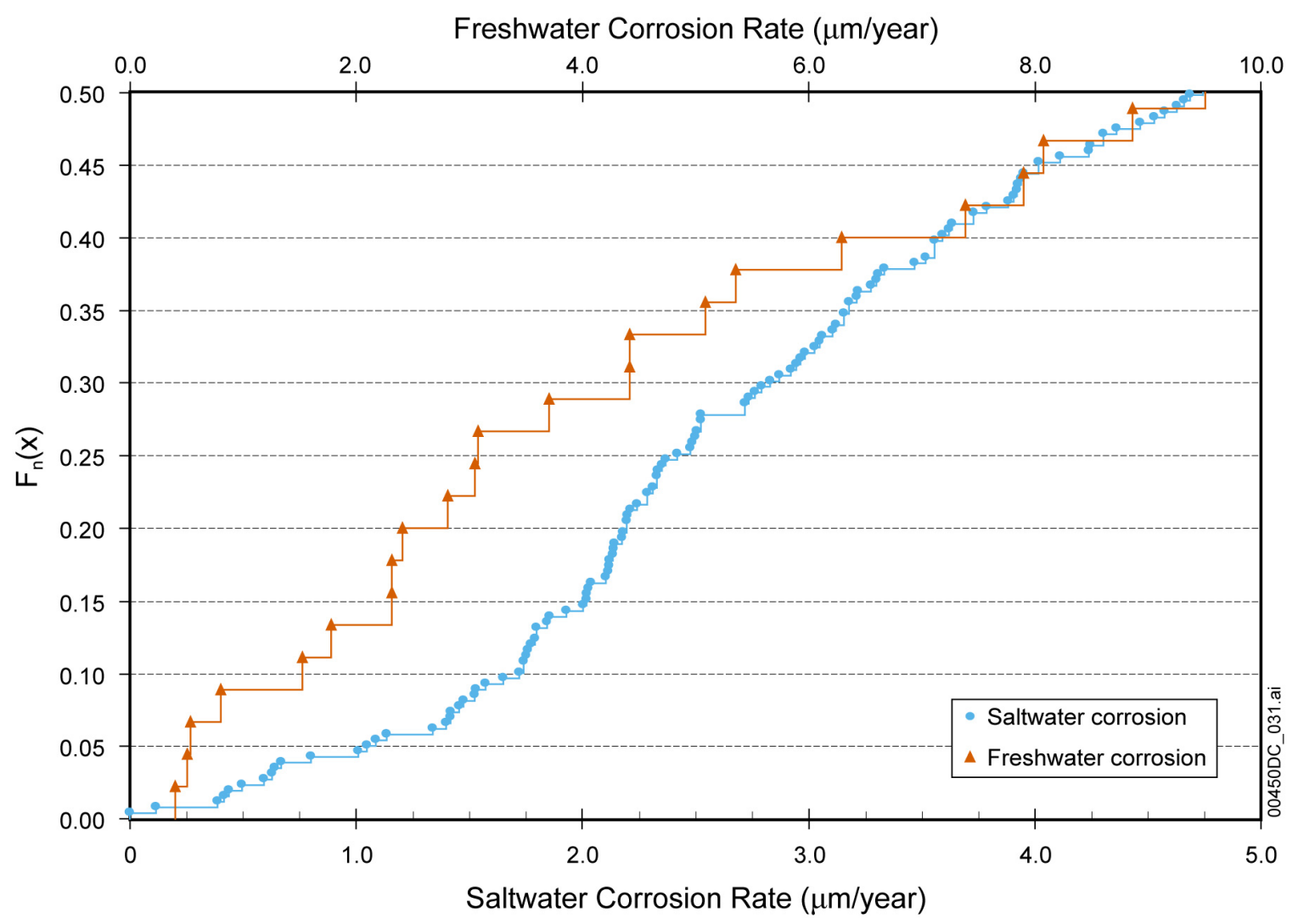

NOTE: ECDF calculated in ECDF_metals.xls in Appendix II from values in Table 4-21 and Table 4-22. Archived in output DTN: MO0409SPAACRWP.000.

Figure 6-15. ECDF for Aluminum Alloy For $F_{n}(x)$ Less than 0.50 


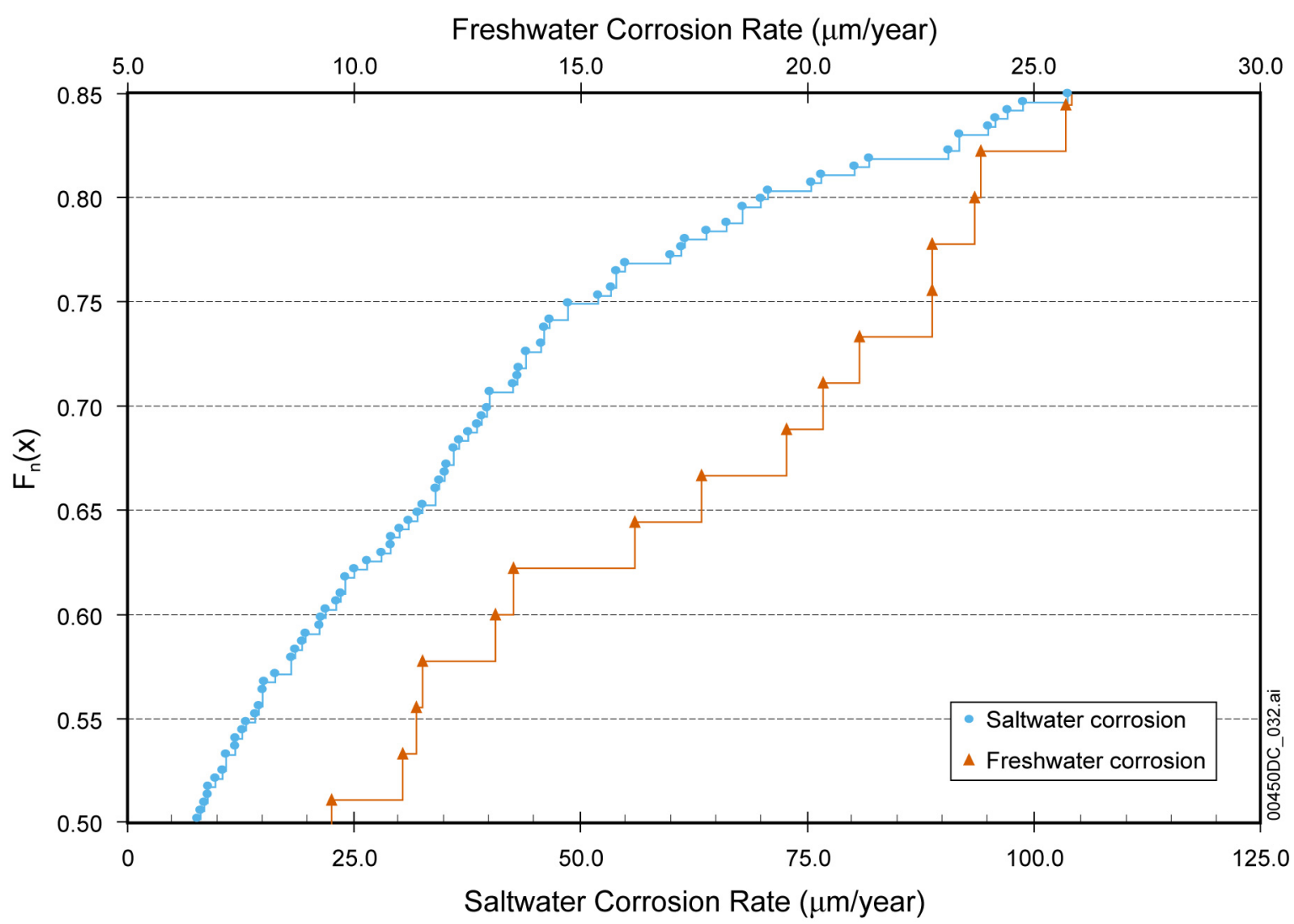

NOTE: $\quad$ ECDF calculated in ECDF_metals.xls in Appendix II from values in Table 4-21 and Table 4-22. Archived in output DTN: MO0409SPAACRWP.000.

Figure 6-16. ECDF for Aluminum Alloy For $F_{n}(x)$ Between 0.50 and 0.85

Table 6-11. Minimum, Maximum, Mean, and Median Aqueous Corrosion Rates of Aluminum Alloy

\begin{tabular}{|l|c|c|c|c|c|}
\hline \multirow{2}{*}{ Environment } & \multicolumn{4}{|c|}{ Corrosion Rate $(\mu \mathrm{m} / \mathrm{yr})$} & \multirow{2}{*}{$\begin{array}{c}\text { Standard } \\
\text { Deviation }\end{array}$} \\
\cline { 2 - 6 } & Minimum & Maximum & Median & Mean & 10.84 \\
\hline Freshwater & 0.40 & 36.93 & 9.50 & 12.95 & 15.34 \\
\hline Saltwater & 0.12 & 110.91 & 4.76 & 9.69 & \\
\hline
\end{tabular}

NOTE: Calculated in aluminum_.x/s in Appendix II from values in Table 4-21 and Table 4-22. Archived in output DTN: MO0409SPAACRWP.000. 


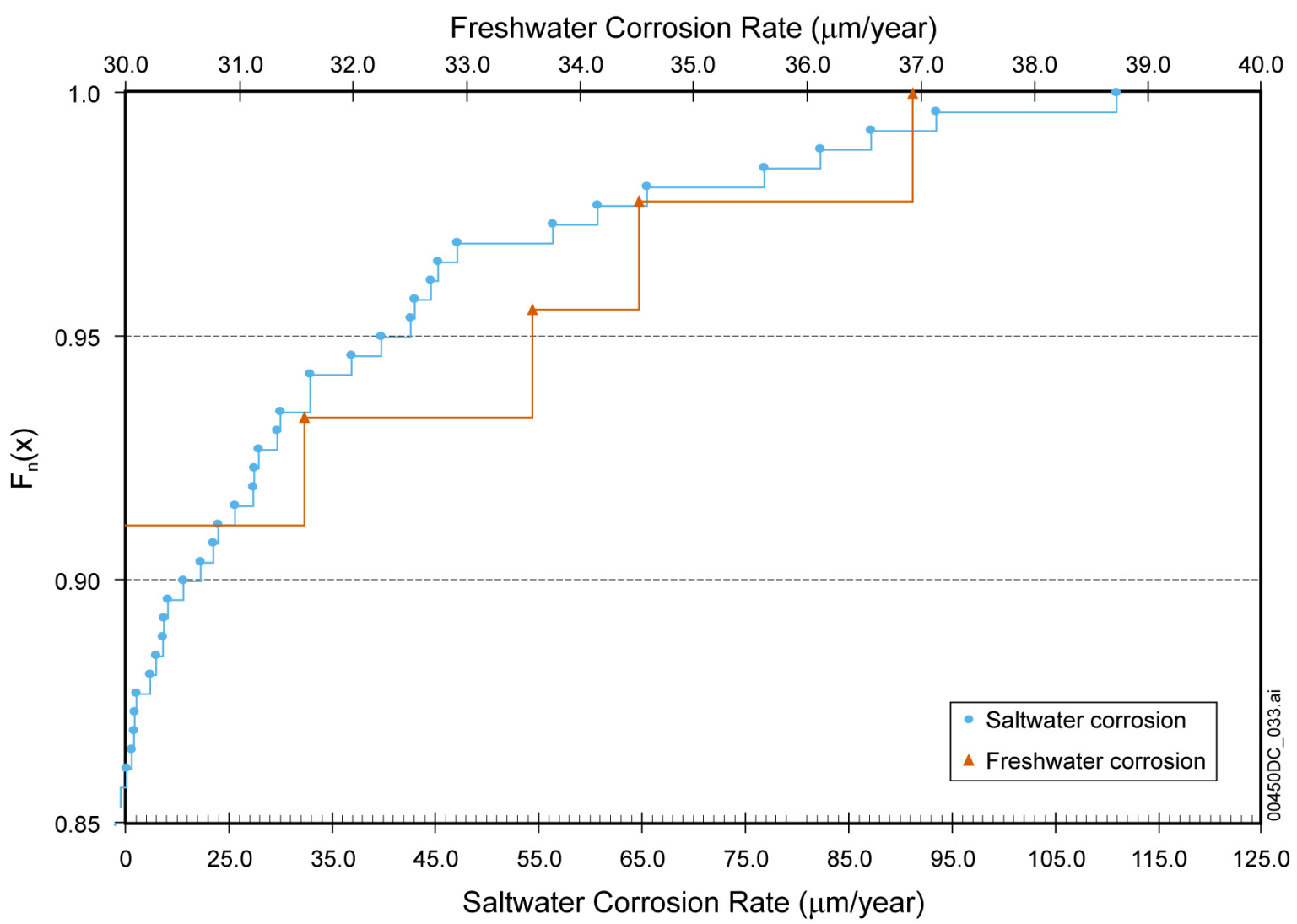

NOTE: $\quad$ ECDF calculated in ECDF_metals.xls in Appendix II from values in Table 4-21 and Table 4-22. Archived in output DTN: MO0409SPAACRWP.000.

Figure 6-17. ECDF for Aluminum Alloy For $F_{n}(x)$ Between 1.0 and 0.85

\subsubsection{Zircaloy (2 and 4)}

As mentioned in Section 4.1.3.5, most information on the corrosion of zircaloy is from high temperature experiments in which the weight gain over time is measured (i.e., Hillner et al. 1998 [DIRS 100455]; Rothman 1984 [DIRS 100417]). Hillner et al. (1998 [DIRS 100455]) conclude that oxide growth in the Yucca Mountain repository over a period of one million years would be extremely small $(0.3 \mathrm{mils}, 1 \mathrm{mil}=25.4 \mu \mathrm{m})$. The data for the corrosion of zircaloy at low temperatures $\left(21.5^{\circ} \mathrm{C}\right.$ to $\left.43^{\circ} \mathrm{C}\right)$ recorded by Uziemblo and Smith (1989 [DIRS 101231]) corroborates this by indicating a degradation rate of zero for all experiments in dilute solution. Rothman (1984 [DIRS 100417]) summarizes data by other authors for a case with a constant temperature of $180^{\circ} \mathrm{C}$ for 10,000 years, which yields a depth of oxidized zircaloy ranging from 4 to $53 \mu \mathrm{m}$ with an average of $17 \mu \mathrm{m}$. However, the repository is not expected to remain at temperatures near $180^{\circ} \mathrm{C}$ for 10,000 years (BSC 2004 [DIRS 168848], Section 6.5.5.1). Therefore, for the purposes of this analysis, this metal is considered insoluble and, consequently, has no associated statistical analysis.

\subsubsection{Alloy N06464}

Corrosion data for a Ni-Cr-Mo-Gd alloy-Alloy N06464 (presented in Table 4-23) shows the metal to hold up well to corrosion. These data are presented in Figure 6-18. Statistical 
information on the corrosion of Alloy N06464 is located in Figure 6-19 and Table 6-12. The first point at 0.03 years $(415.8 \mathrm{~nm} / \mathrm{yr})$ is not used as it is not consistent with the rest of the data.

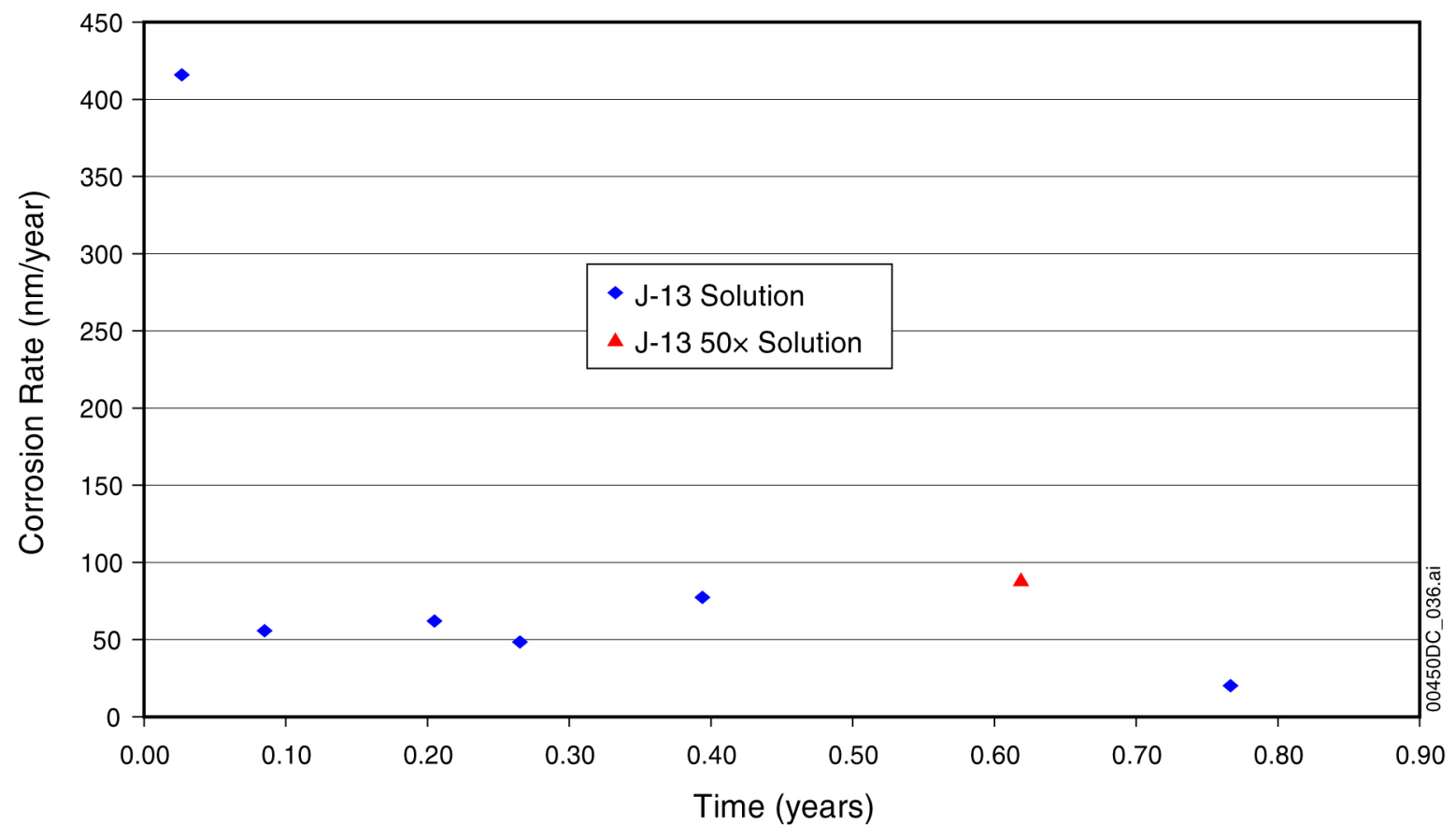

NOTE: Graphical representation of values in Table 4-23. Graphed in NiCrMoGdalloy.xls in Appendix II. Archived in output DTN: MO0409SPAACRWP.000.

Figure 6-18. Corrosion of Alloy N06464

Table 6-12. Minimum, Maximum, Mean, and Median Corrosion Rates of Alloy N06464 in Solution

\begin{tabular}{|l|c|c|c|c|c|}
\hline \multirow{2}{*}{ Environment } & \multicolumn{4}{|c|}{ Corrosion Rate (nm/yr) } & \multirow{2}{*}{$\begin{array}{c}\text { Standard } \\
\text { Deviation }\end{array}$} \\
\cline { 2 - 6 } & Minimum & Maximum & Median & Mean & 21.1 \\
\hline Freshwater & 20.1 & 77.4 & 55.7 & 52.8 & \\
\hline
\end{tabular}

NOTE: Calculated in NiCrMoGdalloy.x/s in Appendix II from values in Table 4-23; archived in output DTN: MO0409SPAACRWP.000. 


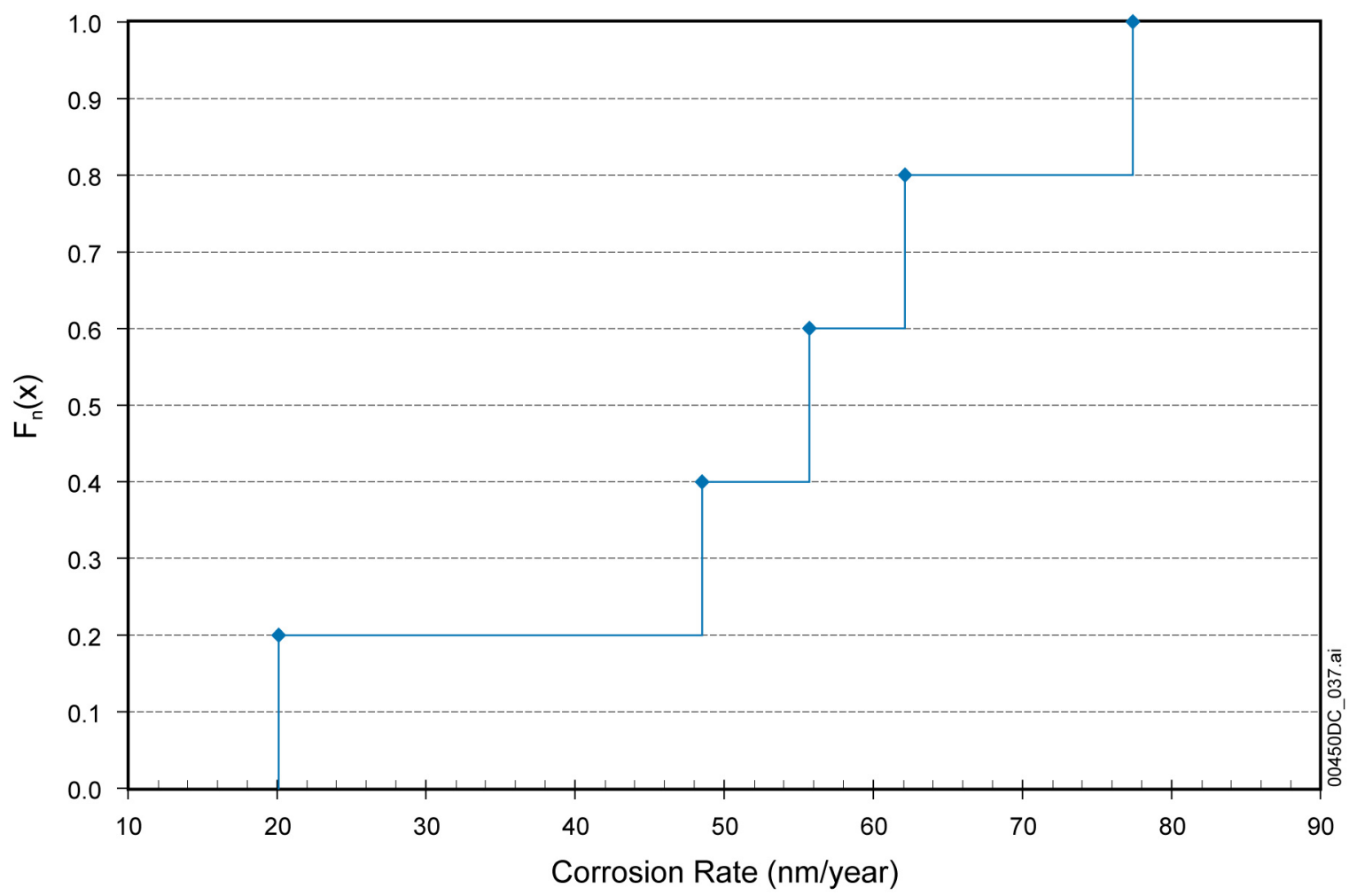

NOTE: $\quad$ ECDF calculated in ECDF_metals.xls in Appendix II from values in Table 4-23; archived in output DTN: MO0409SPAACRWP.000.

Figure 6-19. ECDF for Alloy N06464 in Freshwater at Time $\leq 0.8$ Years

\subsubsection{Inconel Alloys}

Due to the lack of corrosion data on Inconel Alloy 600 and X-750, Inconel Alloy 625 was used as a surrogate for Inconel Alloys X-750 and 600. This approach is reasonable since the composition of Inconel Alloys X-750 and 600 is similar to that of Inconel Alloy 625 (Section 5.2.1). The most in-depth study found for the corrosion of Inconel Alloy 625 is Corrosion Data and Modeling, Update for Viability Assessment (McCright 1998 [DIRS 114637]). Given the short duration of the tests (one year), the values for the corrosion rates in Table 4-24 through Table 4-26 might be slightly high for long-term predictions. The statistical information on the corrosion of Inconel alloys is located in Table 6-13 and Figure 6-20. 


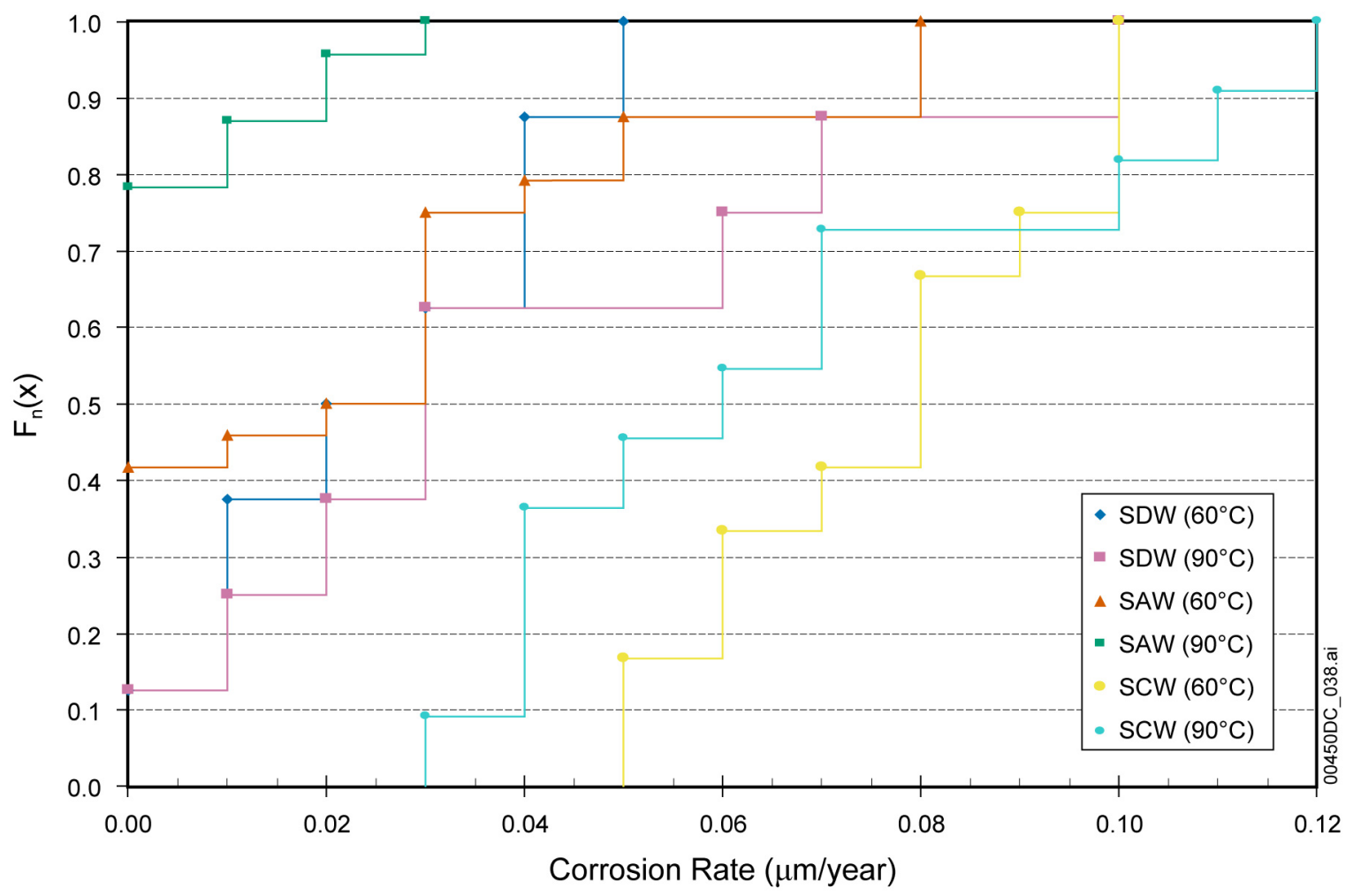

NOTE: $\quad$ ECDF calculated in ECDF_metals.x/s in Appendix II from values in Table 4-24 through Table 4-26. Archived in DTN: MO0409SPAACRWP.000.

Figure 6-20. ECDF for Inconel Alloys in All Water Types Investigated

Table 6-13. Minimum, Maximum, Mean, and Median Aqueous Corrosion Rates of Inconel Alloys

\begin{tabular}{|c|c|c|c|c|c|}
\hline \multirow[b]{2}{*}{ Environment } & \multicolumn{4}{|c|}{ Corrosion Rate $(\mu \mathrm{m} / \mathrm{yr})$} & \multirow{2}{*}{$\begin{array}{l}\text { Standard } \\
\text { Deviation }\end{array}$} \\
\hline & Minimum & Maximum & Median & Mean & \\
\hline $\operatorname{SDW}\left(60^{\circ} \mathrm{C}\right)$ & 0.00 & 0.05 & 0.025 & 0.025 & 0.018 \\
\hline $\operatorname{SDW}\left(90^{\circ} \mathrm{C}\right)$ & 0.00 & 0.10 & 0.030 & 0.040 & 0.034 \\
\hline $\operatorname{SAW}\left(60^{\circ} \mathrm{C}\right)$ & 0.00 & 0.08 & 0.025 & 0.027 & 0.027 \\
\hline SAW $\left(90^{\circ} \mathrm{C}\right)$ & 0.00 & 0.03 & 0.00 & 0.004 & 0.008 \\
\hline $\operatorname{SCW}\left(60^{\circ} \mathrm{C}\right)$ & 0.05 & 0.10 & 0.08 & 0.077 & 0.019 \\
\hline $\operatorname{SCW}\left(90^{\circ} \mathrm{C}\right)$ & 0.03 & 0.12 & 0.06 & 0.066 & 0.031 \\
\hline
\end{tabular}

NOTE: $\quad$ Calculated in aqueous Inconel-Alloy_.x/s in Appendix II from values in Table 4-24 through Table 4-26; archived in output DTN: MO0409SPAACRWP.000.

\subsubsection{Alloy 22}

Corrosion rate information for Alloy 22 can be found in General Corrosion and Localized Corrosion of Waste Package Outer Barrier (BSC 2003 [DIRS 166834]). 


\subsection{MISCELLANEOUS MATERIALS}

Section 6.3 contains the rate analyses of the sintered compounds and $\mathrm{GdPO}_{4}$. Values for $25^{\circ} \mathrm{C}$ were extrapolated from high temperature rate information. The extrapolated values are provided for comparison to previously utilized rates rather than attempting a statistical analysis.

\subsubsection{Silicon Carbide (SiC) Oxidation}

For SiC corrosion, the following equation was suggested by Rechard (1995 [DIRS 101084], Section 11.1.3, Equation 11.1.3-3) to use when the surface area of the material is difficult to estimate. The equation applies to both humid and wet oxic conditions (Section 11.3, for "TRISO Coating for Metal Carbide Fuel").

$$
M=A \mathrm{e}^{-B / T}\left(t_{2}{ }^{C}-t_{1}{ }^{C}\right) D E M_{\text {layer }}
$$

where:

$$
\begin{array}{ll}
M & =\text { Mass of layer corroded in time step }(\mathrm{kg}) \\
A & =\text { Arrhenius-type pre-exponential term }(1 / \mathrm{s}) \\
B & =\text { Arrhenius-type activation energy term }(\mathrm{K}) \\
T & =\text { Temperature of the material }(\mathrm{K}) \\
t_{2}, t_{1} & =\text { Time at beginning and end of time step }(\mathrm{s}) \\
C & =\text { Time-dependent term }(-) \\
D & =\text { Saturation-dependence term }(-) \\
E & =\text { Oxygen Concentration-dependence term }(-) \\
M_{\text {layer }} & =\text { Mass of layer at time zero }(\mathrm{kg}) .
\end{array}
$$

Sections 11.3 and 11.4 of Rechard (1995 [DIRS 101084]) indicate that $A=3 \times 10^{-12}, B=0, C=1$, $D=1$, and $E=0.2$. The Arrhenius-type term is set at $3.0 \times 10^{-12}$ based on the assumption by Rechard (1995 [DIRS 101084]) that TRISO will not fail in the first 10,000 years after repository closure. Lotts et al. (1992 [DIRS 164916]) indicate that TRISO particles will only breach (SiC layer breached) under "unknown mechanisms." DOE Spent Nuclear Fuel Information in Support of TSPA-SR (DOE 2002 [DIRS 158405]), using the equation above, reports a corrosion rate of $6.0 \times 10^{-13} \mathrm{~kg} / \mathrm{m}^{2} \cdot \mathrm{sec}$. However, the results of the equation above are a mass loss ratio, $6.0 \times 10^{-13}$ mass corroded/total mass layer $\left(M / M_{\text {layer }}\right)$, and not an actual corrosion rate.

Most of the studies on the oxidation of $\mathrm{SiC}$ are done in excess of $1,000^{\circ} \mathrm{C}$ in humid air environments. These studies (Jorgensen et al. 1959 [DIRS 164912]; Opila 1999 [DIRS 155502]) show that even at these temperatures, $\mathrm{SiC}$ holds up well to oxidation and is still a good barrier. For comparison, the high temperature data of Opila (1999 [DIRS 155502]) are extrapolated in Misc_Materials.xls (Appendix II). These values are archived in output DTN: MO0409SPAACRWP.000 and give an approximate calculated rate of oxidation at $25^{\circ} \mathrm{C}$ of $1.61 \times 10^{-9} \mu \mathrm{m} / \mathrm{yr}$ (equal to an EQ6 rate of $1.61 \times 10^{-22} \mathrm{~mol} / \mathrm{cm}^{2} \cdot \mathrm{s}$ ). In agreement with this calculated value, Lide (2002 [DIRS 160832], p. 4-82) indicates that $\mathrm{SiC}$ is essentially insoluble in water at $25^{\circ} \mathrm{C}$. Therefore, $\mathrm{SiC}$ is considered insoluble for the purposes of this analysis and, consequently, has no statistical analysis associated with it. 


\subsubsection{Pyrolytic Carbon and Graphite Oxidation}

Graphite and pyrolytic carbon are also resistant to oxidation (Section 4.1.4, Table 4-28). The $25^{\circ} \mathrm{C}$ extrapolated values of graphite oxidation are located in Table 6-14 (Appendix II, Misc_Materials.xls). The values are archived in output DTN: MO0409SPAACRWP.000. Propp (1998 [DIRS 149395]) indicates that oxidation of graphite in water should be slower than air oxidation. However, Gurwell (1981 [DIRS 164911]) indicates a rate for water oxidation faster than that indicated for air oxidation by Lotts et al. (1992 [DIRS 164916]) and Propp (1998 [DIRS 149395]). These rates are for experiments carried out in deionized water and may be faster than oxidation in natural waters. However, values for both air and water oxidation indicate an extremely low oxidation rate for this material. Therefore, for the purposes of this analysis, these substances are also considered insoluble. Consequently, graphite and pyrolytic carbon have no statistical analysis associated with them.

Table 6-14. Approximate Values of Oxidation for Graphite and Pyrolytic Carbon

\begin{tabular}{|l|c|c|c|}
\hline & $\begin{array}{c}\text { From Extrapolation of } \\
\text { Lotts et al. 1992 } \\
\text { [DIRS 164916] data }\end{array}$ & $\begin{array}{c}\text { From Extrapolation of } \\
\text { Gurwell 1981 } \\
\text { [DIRS 164911] data }\end{array}$ & $\begin{array}{c}\text { From Extrapolation of } \\
\text { Propp 1998 } \\
\text { [DIRS 149395] Data }\end{array}$ \\
\hline$\mu \mathrm{m} / \mathrm{yr}$ & $1.81 \times 10^{-12}$ & $5.62 \times 10^{-6}$ & $9.59 \times 10^{-10}$ \\
\hline $\begin{array}{l}\text { Equivalent EQ6 Rate } \\
\left(100 \mathrm{~g}-\mathrm{mol} / \mathrm{cm}^{2} \cdot \mathrm{s}\right)\end{array}$ & $1.26 \times 10^{-25}$ & $3.92 \times 10^{-19}$ & $6.69 \times 10^{-23}$ \\
\hline
\end{tabular}

NOTE: Values calculated in Misc_Materials.xls in Appendix II. Archived in output DTN: MO0409SPAACRW̄P.000.

\subsubsection{Gadolinium Phosphate $\left(\mathrm{GdPO}_{4}\right)$ Dissolution}

Natural analogue data (Boatner and Sales 1988 [DIRS 164908]) and data from manufacturers (Lansdowne n.d. [DIRS 164914]) indicate that gadolinium phosphate is essentially insoluble at low temperatures and pressures. However, several studies indicate that the material corrodes in harsh environments or when containing impurities. Tests on synthetic monazite mixed with 20 $\mathrm{wt} \%$ Savannah River defense waste indicate that $<0.001 \mathrm{~g} / \mathrm{m}^{2}$.day of the lanthanide was released from the material and that the monazite matrix had a corrosion rate of less than $0.002 \mathrm{~g} / \mathrm{m}^{2} \cdot$ day in water at $90^{\circ} \mathrm{C}$ (Boatner and Sales 1988 [DIRS 164908], Figure 23 and Section 8.2). This translates into a corrosion rate of approximately $0.12 \mu \mathrm{m} / \mathrm{yr}$ (Appendix II, Misc_Materials.xls). Values are archived in output DTN: MO0409SPAACRWP.000. Van Konynenburg et al. (1998 [DIRS 100948]) report the dissolution of $\mathrm{GdPO}_{4}$ at $0.19 \mathrm{~mm} / \mathrm{yr}$ after 96-hour exposure to highly corrosive media containing 0.01 molar concentrations of radiolysis products (nitric acid and hydrogen peroxide) and chloride buffered at $\mathrm{pH}$ 4. In-Package Chemistry Abstraction (BSC 2004 [DIRS 167621], Appendix III) has shown that lower concentrations of nitric acid and hydrogen peroxide will be produced by radiolysis, having little effect on the $\mathrm{pH}$ and chemistry inside the waste package. Therefore, the test conditions used by Van Konynenburg et al. (1998 [DIRS 100948]) are not representative of expected repository conditions and their rate value was not used for this analysis. For the purpose of this analysis, $\mathrm{GdPO}_{4}$ is considered insoluble since there is strong natural analogue evidence for extreme stability at earth surface temperature and pressure. Therefore, $\mathrm{GdPO}_{4}$ has no statistical analysis associated with it. 


\subsection{SPENT FUELS AND WASTE FORMS}

\subsubsection{DOE Spent Nuclear Fuel}

Dissolution rate models for these fuel types are presented in DSNF and Other Waste Form Degradation Abstraction (BSC 2004 [DIRS 167618], Section 6.3).

\subsubsection{Commercial Spent Nuclear Fuel}

A CSNF degradation rate model is developed and presented in CSNF Waste Form Degradation: Summary Abstraction (BSC 2004 [DIRS 169987]).

\subsubsection{Defense High-Level Waste Glass}

\subsubsection{Fracture Factor}

In EQ6 calculations (i.e., BSC 2003 [DIRS 169107] and BSC 2001 [DIRS 157640]) the geometric surface area of the glass was increased by a factor of 21 to account for fractures due to cooling and dropping of the glass pour canister during loading. This value was used for consistency with Total System Performance Assessment-Viability Assessment (TSPA-VA) Analyses Technical Basis Document (CRWMS M\&O 1998 [DIRS 100362], p. 6-79). However, in that document, the fracture factor of 21 is an average taken over 100 glass pour canisters and does not take into account loading of damaged canisters into a waste package containing only 5 glass pour canisters. glass-fracturefac.xls (Appendix II) uses the same technique as presented in that document to calculate averaged increase of surface area. This technique is based on determining the increase in surface area by thermal cracking of canistered glass that is cooled by free air convection and for impact cracking using the following equation.

( $\{$ impact cracking $\times$ thermal cracking $\times$ percentage of canisters damaged in a drop $\}+\{$ thermal cracking $\times$ percentage of canisters that have not been damaged in a drop $\}$ )

However, the fracture factor determinations in this analysis (see glass-fracturefac.xls in Appendix II) account for there only being 5 canisters per waste package. The calculations in this analysis also account for only a fraction of the surfaces being available for reaction. For more information on the glass fracture factor see Sections 5.4.1 through 5.4.4.

The probability of loading damaged glass pour canisters is calculated with a binomial distribution (Table 6-15) using the assumed loading of damaged glass pour canisters, number of glass pour canisters damaged in a drop, and 5 glass pour canisters per waste package. Total System Performance Assessment-Viability Assessment (TSPA-VA) Analyses Technical Basis Document (CRWMS M\&O 1998 [DIRS 100362], p. 6-79) assumes that 1 in every 100 glass pour canisters will be dropped and damaged during loading. However, the probabilities of 1 in 250, 1 in 500, and 1 in 1,000 glass pour canisters being dropped are also displayed here since safeguards could presumably be implemented to reduce the occurrence of dropped glass pour canisters. These distributions are then used to determine the probabilities of the fracture factor as indicated below. 
Table 6-15. Probability of Loading Damaged Glass Pour Canisters into Waste Packages.

\begin{tabular}{|l|c|c|c|c|}
\hline \multirow{2}{*}{} & \multicolumn{3}{|c|}{ Number of GPCs Dropped While Loading } \\
\cline { 2 - 5 } & 1 in 100 & 1 in 250 & 1 in 500 & 1 in 1,000 \\
\cline { 2 - 5 } & \multicolumn{3}{|c|}{ Probability of Waste Package Loading } \\
\hline $\begin{array}{l}\text { Waste Packages } \\
\text { Containing 0 Dropped } \\
\text { GPCs }\end{array}$ & $9.51 \mathrm{E}-01$ & $9.80 \mathrm{E}-01$ & $9.90 \mathrm{E}-01$ & $9.95 \mathrm{E}-01$ \\
\hline $\begin{array}{l}\text { Waste Packages } \\
\text { Containing 1 Dropped } \\
\text { GPC }\end{array}$ & $4.80 \mathrm{E}-02$ & $1.97 \mathrm{E}-02$ & $9.92 \mathrm{E}-03$ & $4.98 \mathrm{E}-03$ \\
\hline $\begin{array}{l}\text { Waste Packages } \\
\text { Containing 2 Dropped } \\
\text { GPCs }\end{array}$ & $9.70 \mathrm{E}-04$ & $1.58 \mathrm{E}-04$ & $3.98 \mathrm{E}-05$ & $9.97 \mathrm{E}-06$ \\
\hline $\begin{array}{l}\text { Waste Packages } \\
\text { Containing 3 Dropped } \\
\text { GPCs }\end{array}$ & $9.80 \mathrm{E}-06$ & $6.35 \mathrm{E}-07$ & $7.97 \mathrm{E}-08$ & $9.98 \mathrm{E}-09$ \\
\hline $\begin{array}{l}\text { Waste Packages } \\
\text { Containing 4 Dropped } \\
\text { GPCs }\end{array}$ & $4.95 \mathrm{E}-08$ & $1.27 \mathrm{E}-09$ & $7.98 \mathrm{E}-11$ & $5.00 \mathrm{E}-12$ \\
\hline $\begin{array}{l}\text { Waste Packages } \\
\text { Containing 5 Dropped } \\
\text { GPCs }\end{array}$ & $1.00 \mathrm{E}-10$ & $1.02 \mathrm{E}-12$ & $3.20 \mathrm{E}-14$ & $1.00 \mathrm{E}-15$ \\
\hline
\end{tabular}

NOTE: Values calculated in glass-fracturefac.xls in Appendix II. Archived in output DTN: MO0404SPAHLWGF.001. $\mathrm{GPC}=$ glass pour canister.

Table 6-16 is a summary of the data in glass-fracturefac.xls in Appendix II. Since the degree of availability of reaction surfaces in fractures to aqueous media for glass degradation is unclear, several options are explored here. These provide the range of possible surface area exposure that may occur inside the glass pour canisters due to fracturing of the high level waste glass.

Since references providing the number of fractures in fractured glass are unavailable, a probability distribution for number of fractures or a direct fracture factor is not possible. However, the probability of a particular fracture factor can be derived indirectly from knowing the number of glass pour canisters damaged in a drop, how many damaged glass pour canisters are loaded into a waste package, and the fraction of fractures available for reaction. Table 6-16 provides a range of possible scenarios since it is unknown exactly how many glass pour canisters will be damaged in drops, how many damaged glass pour canisters will be loaded into any one waste package, and the degree of availability of reaction surfaces in fractures. To use Table 6-16, the user will have to make several assumptions about the parameters. An example of this is if we assume that 1 in every 100 glass pour canisters are damaged in a drop. The likelihood of having two of these damaged glass pour canisters in any one waste package is $9.70 \times 10^{-4}$. Then reading across the table, the only way to get a fracture factor of approximately, as an example, 12.50 is to have all fractures available to aqueous media. Therefore, with the combined variables of 1 in 100 dropped, two damaged glass pour canisters loaded in a single waste package, and all fractures available for reaction, the probability of having this fracture factor is $9.70 \times 10^{-4}$. However, if only half of the fractures are assumed to be available for reaction, the same probability will yield a fracture factor of 6.25 instead of 12.50. The fracture factor of 21 used in previous EQ6 calculations (i.e., BSC 2003 DIRS 169107]; BSC 
2001 [DIRS 157640]) is, therefore, not possible even if all fractures are available and 3 damaged glass pour canisters are loaded into a waste package. This high fracture factor is only possible if 4 or 5 damaged glass pour canisters are loaded into a waste package, which (Table 6-15) has a very small probability.

Table 6-16. Fracture Factor Probabilities (Fracture Factor Averaged Over 5 Glass Pour Canisters) for High Level Waste Glass Based on Loading Damaged Glass Pour Canisters into Waste Packages.

\begin{tabular}{|c|c|c|c|c|c|c|c|}
\hline & \multicolumn{4}{|c|}{ Number of GPCs Dropped While Loading } & \multicolumn{3}{|c|}{$\begin{array}{l}\text { Fraction of total fractures } \\
\text { available for reaction }\end{array}$} \\
\hline & 1 in 100 & 1 in 250 & 1 in 500 & 1 in 1,000 & $\begin{array}{c}1 / 4 \\
\text { available }\end{array}$ & $\begin{array}{c}1 / 3 \\
\text { available }\end{array}$ & $\begin{array}{c}1 / 2 \\
\text { available }\end{array}$ \\
\hline & \multicolumn{4}{|c|}{ Probability of Waste Package Loading } & \multicolumn{3}{|c|}{$\begin{array}{l}\text { Fracture Factor Associated with } \\
\text { Available Fractures }\end{array}$} \\
\hline $\begin{array}{l}\text { WPs containing } 0 \\
\text { dropped GPCs }\end{array}$ & $9.51 \mathrm{E}-01$ & $9.80 \mathrm{E}-01$ & $9.90 \mathrm{E}-01$ & 9.95E-01 & 1 & 1 & 1 \\
\hline $\begin{array}{l}\text { WPs containing } 1 \\
\text { dropped GPC }\end{array}$ & $4.80 \mathrm{E}-02$ & $1.97 \mathrm{E}-02$ & $9.92 \mathrm{E}-03$ & $4.98 \mathrm{E}-03$ & 1.56 & 2.08 & 3.13 \\
\hline $\begin{array}{l}\text { WPs containing } 2 \\
\text { dropped GPCs }\end{array}$ & $9.70 \mathrm{E}-04$ & $1.58 \mathrm{E}-04$ & $3.98 \mathrm{E}-05$ & 9.97E-06 & 3.13 & 4.17 & 6.25 \\
\hline \multirow[t]{4}{*}{$\begin{array}{l}\text { WPs containing } 3 \\
\text { dropped GPCs }\end{array}$} & $9.80 \mathrm{E}-06$ & $6.35 \mathrm{E}-07$ & 7.97E-08 & $9.98 \mathrm{E}-09$ & 4.69 & 6.25 & 9.38 \\
\hline & \multicolumn{4}{|c|}{ Number of GPCs Dropped While Loading } & \multicolumn{3}{|c|}{$\begin{array}{l}\text { Fraction of Total Fractures } \\
\text { Available for Reaction }\end{array}$} \\
\hline & 1 in 100 & 1 in 250 & 1 in 500 & 1 in 1,000 & $\begin{array}{l}2 / 3 \\
\text { available }\end{array}$ & $\begin{array}{l}3 / 4 \\
\text { available }\end{array}$ & $\begin{array}{l}\text { all } \\
\text { available }\end{array}$ \\
\hline & \multicolumn{4}{|c|}{ Probability of waste package loading } & \multicolumn{3}{|c|}{$\begin{array}{l}\text { Fracture factor associated with } \\
\text { available fractures }\end{array}$} \\
\hline $\begin{array}{l}\text { WPs containing } 0 \\
\text { dropped GPCs }\end{array}$ & $9.51 \mathrm{E}-01$ & $9.80 \mathrm{E}-01$ & $9.90 \mathrm{E}-01$ & $9.95 \mathrm{E}-01$ & 1 & 1 & 1 \\
\hline $\begin{array}{l}\text { WPs containing } 1 \\
\text { dropped GPC }\end{array}$ & $4.80 \mathrm{E}-02$ & 1.97E-02 & $9.92 \mathrm{E}-03$ & 4.98E-03 & 4.17 & 4.69 & 6.25 \\
\hline $\begin{array}{l}\text { WPs containing } 2 \\
\text { dropped GPCs }\end{array}$ & $9.70 \mathrm{E}-04$ & $1.58 \mathrm{E}-04$ & $3.98 \mathrm{E}-05$ & 9.97E-06 & 8.33 & 9.38 & 12.50 \\
\hline $\begin{array}{l}\text { WPs containing } 3 \\
\text { dropped GPCs }\end{array}$ & $9.80 \mathrm{E}-06$ & $6.35 \mathrm{E}-07$ & 7.97E-08 & $9.98 \mathrm{E}-09$ & 12.50 & 14.06 & 18.75 \\
\hline
\end{tabular}

NOTE: $\quad$ GPC $=$ Glass Pour Canister, WP $=$ Waste Package.

Values calculated in glass-fracturefac.xls in Appendix II using assumptions presented in Section 5.4. Archived in output DTN: MO0404SPAHLWGF.001.

The fracture factors presented in Table 6-16 were calculated using the assumptions in Section 5.4. All fractures due to only cooling are considered to have no effect on the reactive surface area (Section 5.4.3) so that the fracture factor for any glass within glass pour canisters that have not been dropped is 1 (or geometric surface area). For canisters that have been dropped, the factor of cooling (12.5) and increase in fracture factor due to a drop (5), from Sections 5.4.1 and 5.4.2, are multiplied by the reactivity of fracture surfaces as compared to unrestricted surfaces $(1 / 2$, Section 5.4.4). 
Another approach would be to use fracture factors only for those canisters that are considered to be damaged and use the geometric surface area for those that have not been dropped or otherwise damaged. Fracture factors calculated using this approach are presented in Table 6-17.

Table 6-17. True Fracture Factors for High-Level Waste Glass in One Glass Pour Canister

\begin{tabular}{|c|c|c|c|}
\hline Total Fractures available & Fracture Factor & Total Fractures available & Fracture Factor \\
\hline $1 / 4$ & 7.81 & $2 / 3$ & 20.83 \\
\hline $1 / 3$ & 10.42 & $3 / 4$ & 23.44 \\
\hline $1 / 2$ & 15.63 & All & 31.25 \\
\hline
\end{tabular}

NOTE : Values calculated in glass-fracturefac.x/s in Appendix II using assumptions presented in Section 5.4 . Archived in output DTN: MO0404SPAHLWGF.001.

Defense HLW Glass Degradation Model (BSC 2004 [DIRS 167619]) calculates fracture factors between 4 and 17 with 4 being the most likely and 17 as the maximum. To obtain the maximum value, that document also takes the same approach as Total System Performance AssessmentViability Assessment (TSPA-VA) Analyses Technical Basis Document (CRWMS M\&O 1998 [DIRS 100362]) by taking the average over 100 GPCs (1 in 100 dropped), assuming all glass surfaces are available, and that the increase due to a drop is 40 . The fracture factor of 4 is derived from assuming that only $1 / 2$ of the fractures are available to water and the reactivity in these fractures is half that at the surface of the glass. The value of 40 times increase for glass in damaged glass pour canisters comes from using an impact velocity of 117 feet per second, which carries an equivalent drop height of approximately 213 feet (Smith and Ross 1975 [DIRS 102088], pp. 12 to 14 and Figure 41). This extreme velocity represents velocities associated with severe train accidents. Smith and Ross (1975 [DIRS 102088], pp. 12 to 14 and Figure 41) continue to indicate that $>99 \%$ of freight train accidents occur at velocities lower than 117 feet per second. Also, objects on board trains would experience lower impact velocities than the train, the deformation of the cars and mountings providing cushioning. Therefore this fracture factor of 40 is considered an upper bound. When looking at total repository performance, the fracture factors in Defense HLW Glass Degradation Model (BSC 2004 [DIRS 167619]) would be useful as an upper bound. However, this approach does not provide a suitable fracture factor for calculations dealing with single waste packages.

\subsubsection{2 pH-Dependent Corrosion Rate}

The high-level waste glass degradation rate is a function of a $\mathrm{pH}$-dependent corrosion rate combined with the surface area exposure of the glass. This rate is developed and presented in Defense HLW Glass Degradation Model (BSC 2004 [DIRS 167619]).

\subsection{TITANIUM GRADES 7 AND 24}

\subsubsection{Titanium Grade 7}

Corrosion rate data for Titanium Grade 7 is analyzed in General Corrosion and Localized Corrosion of the Drip Shield (BSC 2004 [DIRS 169845]) and will not be discussed further in this report. The corrosion rates for Titanium Grade 7 are used to calculate the corrosion rates for Titanium Grade 24 in Section 6.5.2. 


\subsubsection{Titanium Grade 24}

Titanium Grade 24 , an alloy with $\sim 6 \mathrm{wt} \% \mathrm{Al}, 4 \mathrm{wt} \% \mathrm{~V}$, and $\sim 0.04$ to $0.08 \mathrm{wt} \% \mathrm{Pd}$, is used as the structural material in the design of the drip shield. An experimentally obtained corrosion rate for Titanium Grade 24 under repository conditions is not available at this time. However, the comparative corrosion behavior of Titanium Grade 24 can be estimated based on available data for other titanium alloys.

General Corrosion and Localized Corrosion of the Drip Shield (BSC 2004 [DIRS 169845]) plots all of the 2.5-year weight-loss data (vapor, aqueous, and water line) for Titanium Grade 7 from DTN: LL030410012251.056 [DIRS 169583] in the CDF shown in Figure 6-21. The corrosion rate from all weight-loss specimens at the $85 \%$ percentile of the cumulative distribution function is about $15 \mathrm{~nm} / \mathrm{yr}$.

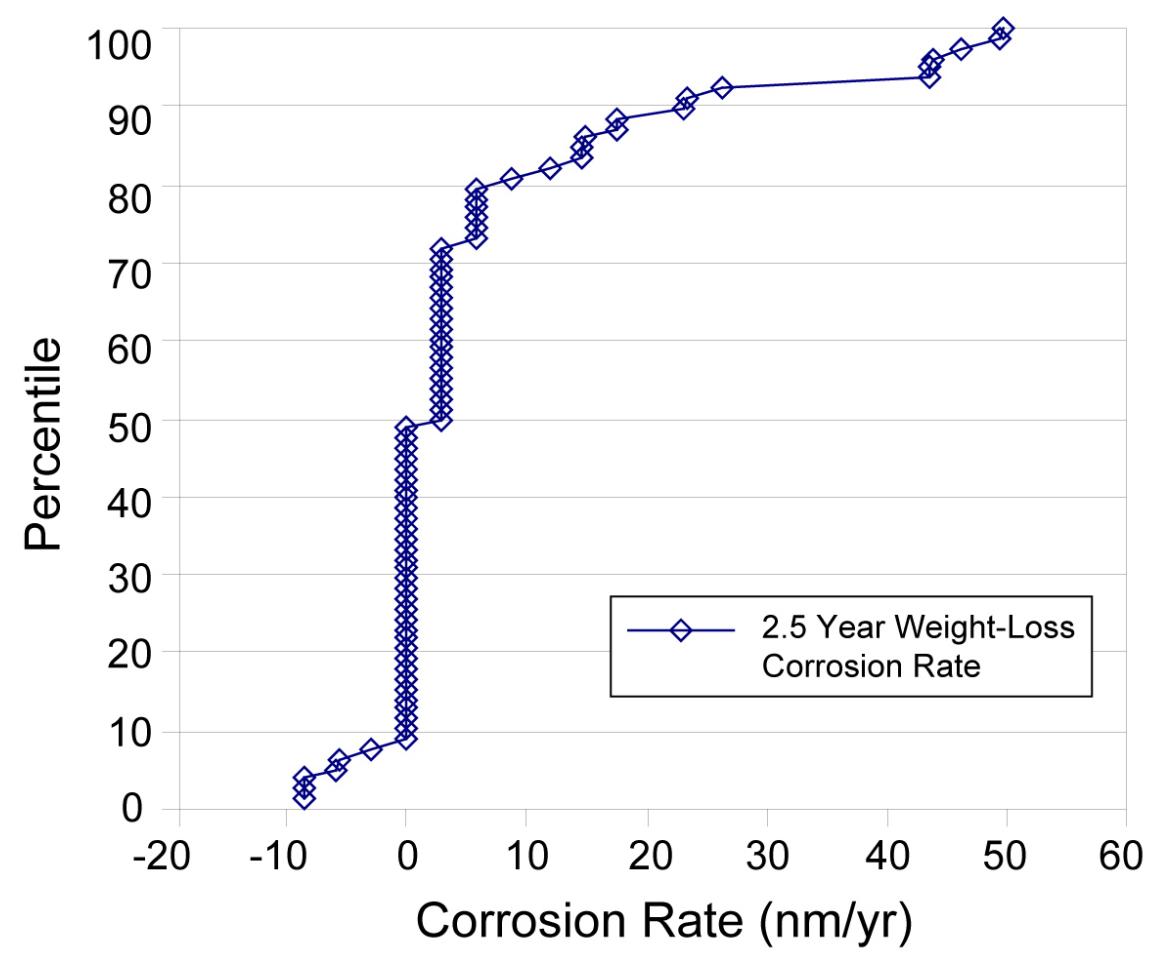

Source: BSC 2004 [DIRS 169845] Figure 26.

Figure 6-21. Titanium Grade 7 Weight-Loss Corrosion Rates after 2.5 Years

The corrosion rates of Titanium Grades 2 (Titanium with no Pd) and 5 (Ti-6Al-4V with no Pd) in hydrochloric acid solutions, a very aggressive test media for titanium alloys, are shown in Figure 6-22 along with those of Titanium Grades 7, 16, and 24. The addition of 0.04 to $0.08 \mathrm{wt} \%$ of Pd to Titanium Grades 2 and 5 (to produce Titanium Grades 16 and 24) significantly improves the corrosion resistance of the alloy as demonstrated in Figure 6-22. From Figure 6-22, it can be seen that the corrosion rate of Titanium Grade 24 is about five times higher than that of Titanium Grade 7 in $3 \%$ boiling $\mathrm{HCl}$. On this basis, a conservative estimate of the corrosion rate of Titanium Grade 24 in less aggressive repository environments would be a corrosion rate five times higher than that of Titanium Grade 7. 


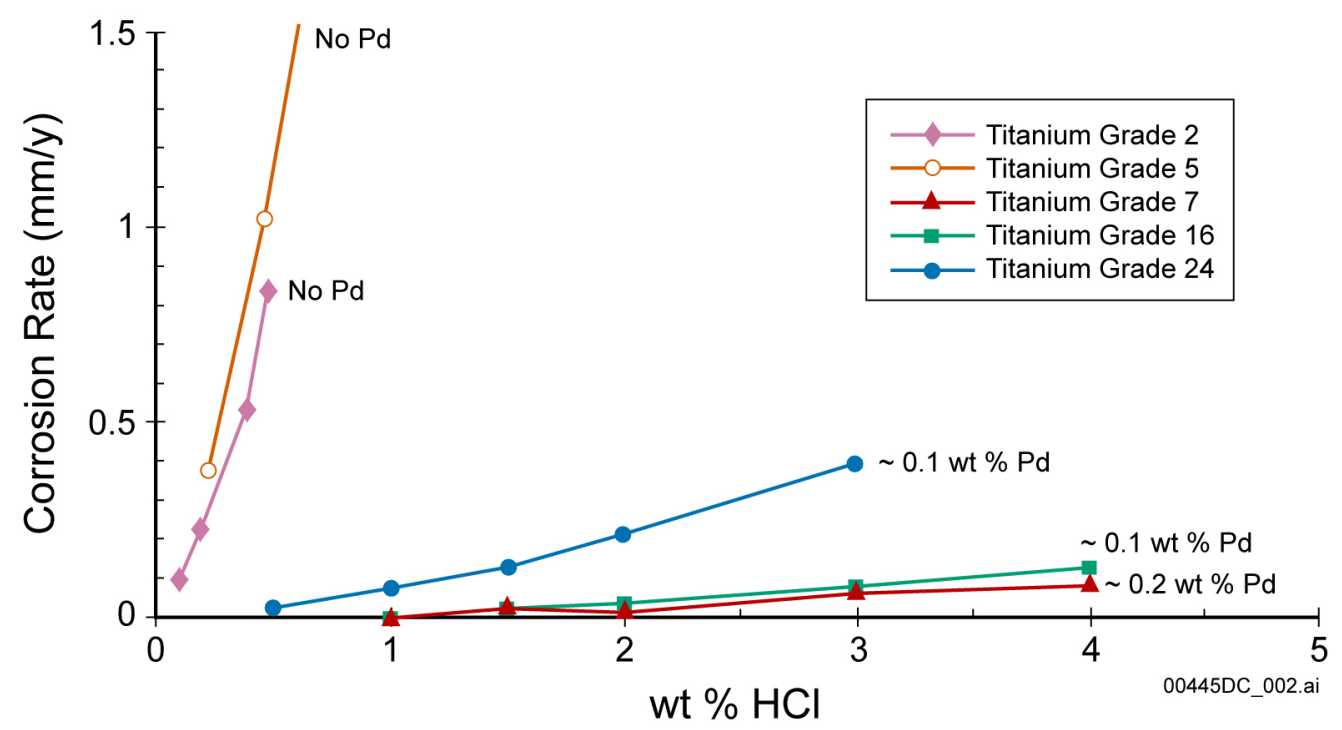

Source: BSC 2004 [DIRS 169847] (Figure 10).

NOTE: This figure shows the significant improvement in corrosion resistance due to addition of palladium.

Figure 6-22. Corrosion Rates of Titanium Grades 2, 5, 7, 16, and 24 in Boiling $\mathrm{HCl}$

A corrosion allowance of $1 \mathrm{~mm}$ per exposed surface is accounted for in the drip shield design (BSC 2004 [DIRS 169220]). The 85th percentile on the cumulative distribution curve (Figure 6-1, based on data collected in all test environments) for the general corrosion rate of Titanium Grade 7 is about $15 \mathrm{~nm} / \mathrm{yr}$. Therefore, the estimated corrosion rate of Titanium Grade 24 at the 85 th percentile is estimated to be a factor of five greater or about $75 \mathrm{~nm} / \mathrm{yr}$. Over a 10,000 -year period, this corrosion rate results in a metal loss of about $0.75 \mathrm{~mm}$ per exposed surface.

Figure 6-23 and Table 6-18 present simple statistical information on the corrosion of Titanium Grade 24 (based on Titanium Grade 7 data from Table 4-31 multiplied by 5 to account for the increased corrosion of Titanium Grade 24 over that of Titanium Grade 7). The information presented below is only for aqueous corrosion, as vapor (or atmospheric) corrosion is not within the scope of this document. Figure 6-23 shows that a corrosion rate of $0.075 \mu \mathrm{m} / \mathrm{yr}(75 \mathrm{~nm} / \mathrm{yr})$ corresponds to the $92^{\text {nd }}$ percentile for the ECDF for corrosion rates in SAW solutions. Over a 10,000 -year period, this corrosion rate results in a metal loss of about $0.75 \mathrm{~mm}$ per exposed surface. 
Table 6-18. Minimum, Maximum, Mean, and Median Corrosion Rates of Titanium Alloy Grade 24 in Solution

\begin{tabular}{|c|c|c|c|c|c|}
\hline \multirow{2}{*}{ Environment } & \multicolumn{4}{|c|}{ Corrosion Rate $(\mu \mathrm{m} / \mathrm{yr})$} & \multirow{2}{*}{$\begin{array}{c}\text { Standard } \\
\text { Deviation }\end{array}$} \\
\cline { 2 - 5 } & Minimum & Maximum & Median & Mean & 0.0301 \\
\hline SAW $\left(60^{\circ} \mathrm{C}\right.$ to $\left.90^{\circ} \mathrm{C}\right)$ & 0.00 & 0.0882 & 0.0183 & 0.00728 & 0.00966 \\
\hline SDW $\left(60^{\circ} \mathrm{C}\right.$ to $\left.90^{\circ} \mathrm{C}\right)$ & 0.00 & 0.0289 & 0.00603 & 0.00 & 0.0837 \\
\hline SCW $\left(90^{\circ} \mathrm{C}\right)$ & 0.0146 & 0.249 & 0.156 & 0.175 & \\
\hline
\end{tabular}

NOTE: Calculated in titanium.x/s in Appendix II from values in Table 4-31; archived in output

DTN: MO0409SPAACRWP.000.

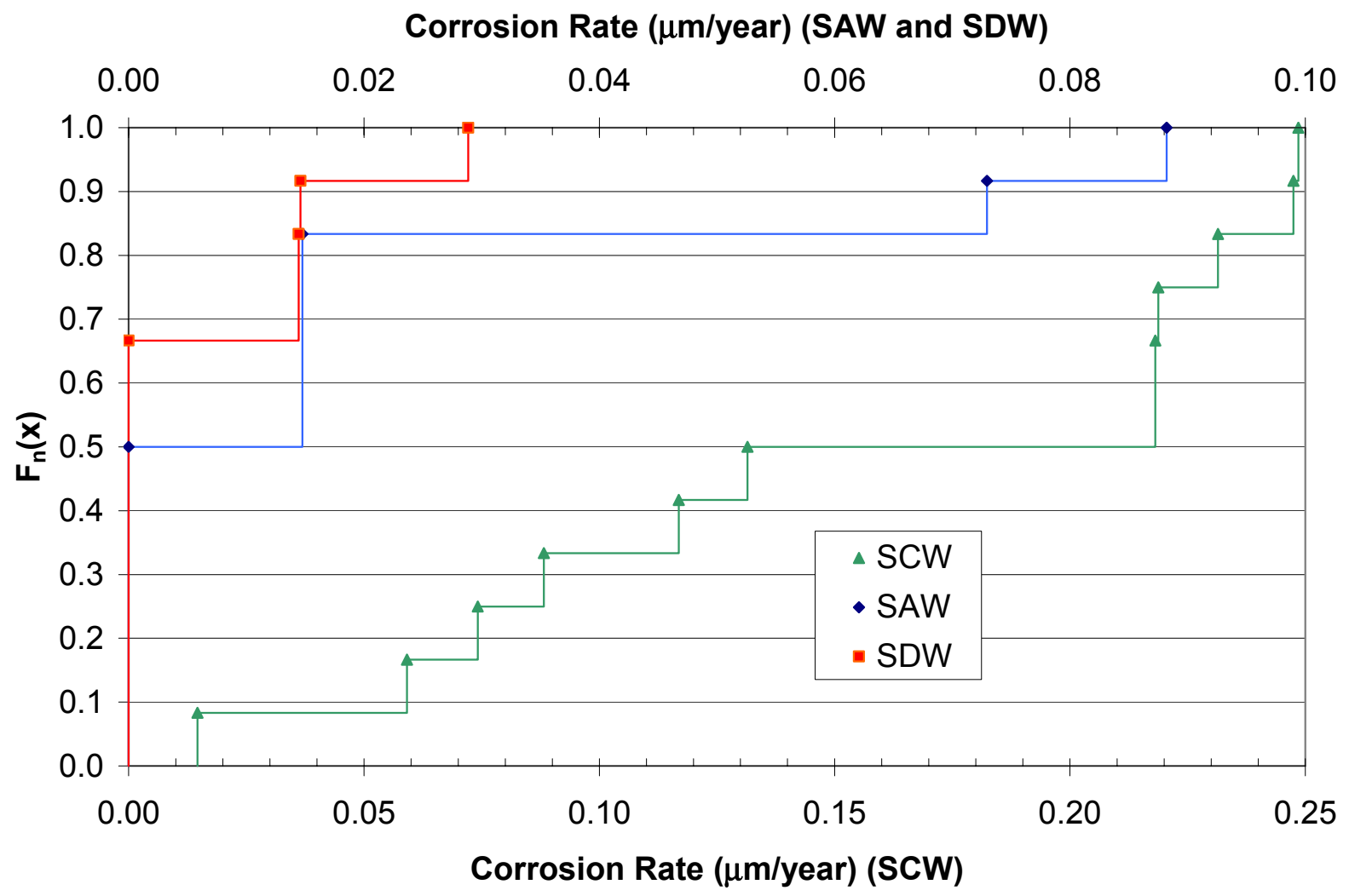

NOTE: Calculated in titanium.x/s in Appendix II from values in Table 4-31; archived in output DTN: MO0409SPAACRWP.000.

Figure 6-23. ECDF for Titanium Grade 24 in All Water Types Investigated 


\section{INTENTIONALLY LEFT BLANK}




\section{CONCLUSIONS}

This analysis presents statistical analyses and ECDFs for aqueous degradation rates affecting waste package material degradation for various DOE SNF types and drip shield materials. These rates are limited by various aqueous parameters such as temperature (up to $100^{\circ} \mathrm{C}$ ), water type (i.e., fresh versus saline), and $\mathrm{pH}$. Corrosion data of materials at $\mathrm{pH}$ extremes (below 4 and above 9) are not included in this analysis as materials commonly display different corrosion behaviors under these conditions. The exception is highly corrosion-resistant materials (Inconel Alloys and Titanium Grade 24) for which rate data from corrosion tests at a $\mathrm{pH}$ of approximately 3 were included.

\subsection{STEELS AND ALLOYS}

Corrosion rates were used to describe statistics of aqueous corrosion of steels and alloys inside the waste package. These rates describe only general corrosion and should not be used for analysis of effects of localized attack on waste package materials. If possible (i.e., if sufficient data existed), the ECDF was used to describe the corrosion data. These are provided in the figures within Section 6. To provide further analysis of the data, the minimum, maximum, mean, and median values (along with their standard deviations) are also presented in Table 7-1. These values are archived in output DTN: MO0409SPAACRWP.000.

Table 7-1. Summary of Statistical Values of Corrosion for Waste Package Metals

\begin{tabular}{|c|c|c|c|c|c|c|}
\hline \multirow[b]{2}{*}{ Metal } & \multirow[b]{2}{*}{ Conditions } & \multicolumn{4}{|c|}{ Corrosion Rate $(\mu \mathrm{m} / \mathrm{yr})$} & \multirow{2}{*}{$\begin{array}{l}\text { Standard } \\
\text { Deviation }\end{array}$} \\
\hline & & Minimum & Maximum & Median & Mean & \\
\hline \multirow{3}{*}{$\begin{array}{l}\text { Stainless Steel } \\
\text { Types } \\
\text { 302/304/304L }\end{array}$} & Freshwater $\left(25^{\circ} \mathrm{C}\right.$ to $\left.100^{\circ} \mathrm{C}\right)$ & 0.001 & 1.570 & 0.1285 & 0.214 & 0.298 \\
\hline & Saltwater $\left(26.7^{\circ} \mathrm{C}\right)$ & 1.588 & 39.147 & 5.08 & 11.441 & 11.134 \\
\hline & Saltwater $\left(90^{\circ} \mathrm{C}\right)$ & 0.660 & 15.900 & 2.03 & 5.816 & 5.953 \\
\hline \multirow{3}{*}{$\begin{array}{l}\text { Stainless Steel } \\
\text { Types } \\
\text { 316/316L/AM-350 }\end{array}$} & Freshwater $\left(29.5^{\circ} \mathrm{C}\right)$ & 0.0007 & 0.0475 & 0.003 & 0.0083 & 0.0136 \\
\hline & Freshwater $\left(50^{\circ} \mathrm{C}\right.$ to $\left.100^{\circ} \mathrm{C}\right)$ & 0.037 & 0.51 & 0.229 & 0.248 & 0.146 \\
\hline & Saltwater $\left(26.7^{\circ} \mathrm{C}\right)$ & 0.0014 & 14.787 & 0.7362 & 1.939 & 3.346 \\
\hline \multirow{3}{*}{ Neutronit } & Freshwater $\left(29.5^{\circ} \mathrm{C}\right)$ & 0.001 & 0.011 & 0.003 & 0.004 & 0.004 \\
\hline & Freshwater $\left(50^{\circ} \mathrm{C}\right.$ to $\left.100^{\circ} \mathrm{C}\right)$ & 0.025 & 0.330 & 0.203 & 0.206 & 0.088 \\
\hline & Saltwater $\left(26.7^{\circ} \mathrm{C}\right)$ & 1.81 & 29.22 & 7.38 & 11.06 & 10.19 \\
\hline \multirow{5}{*}{$\begin{array}{l}\text { Stainless Steel } \\
\text { Type } 304 \text { Alloyed } \\
\text { with } 0.3 \% \text { Boron }\end{array}$} & Ambient-"Fresh" Water & 3.05 & 12.19 & 4.57 & 6.10 & 3.86 \\
\hline & $50^{\circ} \mathrm{C}$-boiling-"Fresh"Water & 3.05 & 27.43 & 16.76 & 15.75 & 7.99 \\
\hline & Ambient-"Harsh" Water & 2.94 & 26.46 & 20.58 & 17.05 & 9.48 \\
\hline & $50^{\circ} \mathrm{C}-"$ Harsh" Water & 38.22 & 147.00 & 88.20 & 94.69 & 42.85 \\
\hline & Boiling-"Harsh" Water & 11.76 & 58.80 & 27.93 & 29.77 & 12.48 \\
\hline \multirow{2}{*}{$\begin{array}{l}\text { Stainless Steel } \\
\text { Type } 304 \text { Alloyed } \\
\text { with } 1.5 \% \text { Boron }\end{array}$} & Ambient-Boiling-"Fresh" Water & 161.54 & 252.98 & 211.84 & 210.31 & 32.77 \\
\hline & Ambient-Boiling-"Harsh" Water & 164.64 & $1,058.40$ & 360.15 & 453.74 & 272.96 \\
\hline \multirow{4}{*}{$\begin{array}{l}\text { Carbon Steel } \\
\text { Type A516 (Times } \\
\text { Less Than } 0.53 \\
\text { Year) }\end{array}$} & $\operatorname{SDW}\left(60^{\circ} \mathrm{C}\right)$ & 78.71 & 130.70 & 101.95 & 102.71 & 12.37 \\
\hline & $\operatorname{SDW}\left(90^{\circ} \mathrm{C}\right)$ & 58.08 & 130.02 & 77.05 & 81.14 & 15.13 \\
\hline & $\operatorname{SCW}\left(60^{\circ} \mathrm{C}\right)$ & 50.25 & 104.20 & 62.77 & 68.08 & 14.17 \\
\hline & $\operatorname{SCW}\left(90^{\circ} \mathrm{C}\right)$ & 7.39 & 22.06 & 12.42 & 12.78 & 3.77 \\
\hline
\end{tabular}


Table 7-1. Summary of Statistical Values of Corrosion for Waste Package Metals (Continued)

\begin{tabular}{|c|c|c|c|c|c|c|}
\hline \multirow{2}{*}{ Metal } & \multirow{2}{*}{ Conditions } & \multicolumn{4}{|c|}{ Corrosion Rate $(\mu \mathrm{m} / \mathrm{yr})$} & \multirow{2}{*}{$\begin{array}{l}\text { Standard } \\
\text { Deviation }\end{array}$} \\
\hline & & Minimum & Maximum & Median & Mean & \\
\hline \multirow{4}{*}{$\begin{array}{l}\text { Carbon Steel } \\
\text { Type A516 (Times } \\
\text { Greater Than } 1.0 \\
\text { Year) }\end{array}$} & $\operatorname{SDW}\left(60^{\circ} \mathrm{C}\right)$ & 65.77 & 106.93 & 74.56 & 77.43 & 8.83 \\
\hline & $\operatorname{SDW}\left(90^{\circ} \mathrm{C}\right)$ & 29.53 & 88.68 & 48.70 & 51.80 & 12.99 \\
\hline & $\operatorname{scW}\left(60^{\circ} \mathrm{C}\right)$ & 6.77 & 14.36 & 10.83 & 10.61 & 2.02 \\
\hline & $\operatorname{SCW}\left(90^{\circ} \mathrm{C}\right)$ & 3.69 & 9.35 & 6.75 & 6.84 & 1.25 \\
\hline \multirow{2}{*}{ Aluminum Alloy } & Freshwater & 0.40 & 36.93 & 9.50 & 12.95 & 10.84 \\
\hline & Saltwater & 0.12 & 110.91 & 4.76 & 9.69 & 15.34 \\
\hline Alloy N06464 & Freshwater & $20.1^{\mathrm{a}}$ & $77.4^{\mathrm{a}}$ & $55.7^{\mathrm{a}}$ & $52.8^{a}$ & $21.1^{\mathrm{a}}$ \\
\hline \multirow{6}{*}{ Inconel Alloy } & $\operatorname{SDW}\left(60^{\circ} \mathrm{C}\right)$ & 0.00 & 0.05 & 0.025 & 0.025 & 0.018 \\
\hline & $\operatorname{SDW}\left(90^{\circ} \mathrm{C}\right)$ & 0.00 & 0.10 & 0.030 & 0.040 & 0.034 \\
\hline & $\operatorname{SAW}\left(60^{\circ} \mathrm{C}\right)$ & 0.00 & 0.08 & 0.025 & 0.026 & 0.026 \\
\hline & $\operatorname{SAW}\left(90^{\circ} \mathrm{C}\right)$ & 0.00 & 0.03 & 0.00 & 0.004 & 0.008 \\
\hline & $\operatorname{scW}\left(60^{\circ} \mathrm{C}\right)$ & 0.05 & 0.10 & 0.08 & 0.077 & 0.019 \\
\hline & $\operatorname{SCW}\left(90^{\circ} \mathrm{C}\right)$ & 0.03 & 0.12 & 0.06 & 0.066 & 0.031 \\
\hline \multirow{3}{*}{$\begin{array}{l}\text { Titanium Alloy } \\
\text { Grade } 24\end{array}$} & $\operatorname{SAW}\left(60^{\circ} \mathrm{C}\right.$ to $\left.90^{\circ} \mathrm{C}\right)$ & 0.00 & 0.0882 & 0.0183 & 0.00728 & 0.0301 \\
\hline & $\operatorname{SDW}\left(60^{\circ} \mathrm{C}\right.$ to $\left.90^{\circ} \mathrm{C}\right)$ & 0.00 & 0.0289 & 0.00603 & 0.00 & 0.00966 \\
\hline & $\operatorname{SCW}\left(90^{\circ} \mathrm{C}\right)$ & 0.0146 & 0.249 & 0.156 & 0.175 & 0.0837 \\
\hline
\end{tabular}

NOTE: ${ }^{a}$ Values are in units of nanometers/year.

Comparison of the change in the rate of Stainless Steel Type 304 and gadolinium-alloyed Stainless Steel Type 304 is presented in (Table 7-2). These values are archived in output DTN: MO0409SPAACRWP.000.

Table 7-2. Comparison of Minimum, Maximum, Mean, and Median Corrosion Rates of GadoliniumAlloyed and Non Gadolinium-Alloyed Stainless Steel Type 304

\begin{tabular}{|c|c|c|c|}
\hline \multirow[b]{2}{*}{ Environment } & \multicolumn{3}{|c|}{ Corrosion Rate ( $\mu \mathrm{m} / \mathrm{yr})$} \\
\hline & Minimum & Maximum & Mean \\
\hline $0.3 \%$ Gd 304 (Ambient-Boiling-"Fresh") ${ }^{a}$ & 3.05 & 15.24 & 9.14 \\
\hline $304 \mathrm{~L}$ Freshwater $\left(25^{\circ} \mathrm{C} \text { to } 100^{\circ} \mathrm{C}\right)^{\mathrm{b}}$ & 0.001 & 1.57 & 0.214 \\
\hline Increase in Corrosion & 3,050 times & 9.71 times & 33.27 times \\
\hline $0.3 \%$ Gd 304 (Ambient-"Harsh") $^{a}$ & 2.94 & 2.94 & 2.94 \\
\hline $\begin{array}{l}\text { Stainless Steel Type 304L Saltwater } \\
\left(<27^{\circ} \mathrm{C}\right)^{\mathrm{b}}\end{array}$ & 1.588 & 39.147 & 11.44 \\
\hline Increase in Corrosion & 1.85 times & 0.08 times & 0.26 times \\
\hline $0.3 \%$ Gd 304 (Boiling-"Harsh") & 5.88 & 23.52 & 11.03 \\
\hline 304L Saltwater $\left(90^{\circ} \mathrm{C}\right)^{\mathrm{b}}$ & 0.66 & 15.9 & 5.816 \\
\hline Increase in Corrosion & 8.91 times & 1.48 times & 1.90 times \\
\hline
\end{tabular}

NOTES: ${ }^{a}$ Values calculated in aqueous-B-Gd-steels.x/s in Appendix II from values in Table 4-12; archived in output DTN: MO0409SPAACRWP.000.

${ }^{\mathrm{b}}$ Values taken from Table 6-4. 


\subsection{MISCELLANEOUS MATERIALS}

Section 6.3 shows that these materials are essentially insoluble. Rates derived from high temperature data (Table 7-3), indicate extremely slow corrosion of these materials. Therefore, they are all considered insoluble for the purposes of this analysis. These values are archived in output DTN: MO0409SPAACRWP.000.

Table 7-3. Summary of Corrosion Behavior and Rates for Graphite, SiC, and Gadolinium Phosphate

\begin{tabular}{|l|c|l|}
\hline \multicolumn{1}{|c|}{ Material } & Rate $(\mu \mathrm{m} / \mathrm{yr})$ & \multicolumn{1}{c|}{ Derived from } \\
\hline \multirow{4}{*}{ Graphite } & $1.84 \times 10^{-12}$ & $\begin{array}{l}\text { From extrapolation data by Lotts et al. 1992 } \\
\text { [DIRS 164916] }\end{array}$ \\
\cline { 2 - 3 } & $5.72 \times 10^{-6}$ & $\begin{array}{l}\text { From extrapolation of Gurwell 1981 } \\
\text { [DIRS 164911] data }\end{array}$ \\
\cline { 2 - 3 } & $9.77 \times 10^{-10}$ & $\begin{array}{l}\text { From extrapolation by Propp 1998 } \\
\text { [DIRS 149395] data }\end{array}$ \\
\cline { 2 - 3 } & Insoluble & $\begin{array}{l}\text { Lide 2002 [DIRS 160832]; Morgan 1981 } \\
\text { [DIRS 164920] }\end{array}$ \\
\hline \multirow{3}{*}{$\mathrm{SiC}$} & $1.61 \times 10^{-9}$ & $\begin{array}{l}\text { From extrapolation of Opila 1999 [DIRS 155502] } \\
\text { data }\end{array}$ \\
\cline { 2 - 3 } & Insoluble & Lide 2002 [DIRS 160832] \\
\hline $\mathrm{GdPO}_{4}$ & Insoluble & $\begin{array}{l}\text { Boatner and Sales 1988 [DIRS 164908], } \\
\text { Lansdowne (n.d. [DIRS 164914]) }\end{array}$ \\
\hline
\end{tabular}

\subsection{GLASS FRACTURE FACTOR}

The corrosion rate of the defense high-level waste glass is dependent on two parameters: a $\mathrm{pH}$ dependent dissolution rate combined with a fracture factor. Most references dealing with fractures discuss how surface area increases. To obtain a fracture factor, more information in the form of availability of fracture surfaces and reactivity of fracture surfaces must be taken into account. Since references do not take these additional parameters into account, surface area measurements represent a total surface area instead of an effective surface area. Therefore, a probability distribution for number of fractures or a direct fracture factor is not possible. However, the probability of a particular fracture factor can be derived indirectly from knowing the number of glass pour canisters damaged in a drop, how many damaged glass pour canisters are loaded into a waste package, and the percentage of fractures available for reaction. Table 7-4 exhibits the probability of a fracture factor based on how many glass pour canisters are loaded into a waste package including those from previous calculations where the fracture factor was averaged over all five glass logs within a waste package. However, using another approach, calculations should account for there being undamaged glass pour canisters in the waste package and apply a fracture factor to only those that are damaged. These values can be found in Table 7-5. Values for the fracture factor are archived in output DTN: MO0404SPAHLWGF.001. 
Table 7-4. Fracture Factor Probabilities (Fracture Factor Averaged Over 5 Glass Pour Canisters) for High-Level Waste Glass Based on Loading Damaged Glass Pour Canisters into Waste Packages

\begin{tabular}{|c|c|c|c|c|c|c|c|}
\hline & \multicolumn{4}{|c|}{ Number of GPCs Dropped While Loading } & \multicolumn{3}{|c|}{$\begin{array}{l}\text { Amount of total fractures } \\
\text { available for reaction }\end{array}$} \\
\hline & 1 in 100 & 1 in 250 & 1 in 500 & 1 in 1,000 & $\begin{array}{c}1 / 4 \\
\text { available }\end{array}$ & $\begin{array}{c}1 / 3 \\
\text { available }\end{array}$ & $\begin{array}{c}1 / 2 \\
\text { available }\end{array}$ \\
\hline & \multicolumn{4}{|c|}{ Probability of waste package loading } & \multicolumn{3}{|c|}{$\begin{array}{c}\text { Fracture factor associated with } \\
\text { available fractures }\end{array}$} \\
\hline $\begin{array}{l}\text { WPs containing } 0 \\
\text { dropped GPCs }\end{array}$ & $9.51 \mathrm{E}-01$ & $9.80 \mathrm{E}-01$ & $9.90 \mathrm{E}-01$ & 9.95E-01 & 1 & 1 & 1 \\
\hline $\begin{array}{l}\text { WPs containing } 1 \\
\text { dropped GPC }\end{array}$ & 4.80E-02 & 1.97E-02 & 9.92E-03 & 4.98E-03 & 1.56 & 2.08 & 3.13 \\
\hline $\begin{array}{l}\text { WPs containing } 2 \\
\text { dropped GPCs }\end{array}$ & $9.70 \mathrm{E}-04$ & $1.58 \mathrm{E}-04$ & $3.98 \mathrm{E}-05$ & $9.97 \mathrm{E}-06$ & 3.13 & 4.17 & 6.25 \\
\hline $\begin{array}{l}\text { WPs containing } 3 \\
\text { dropped GPCs }\end{array}$ & $9.80 \mathrm{E}-06$ & $6.35 \mathrm{E}-07$ & 7.97E-08 & $9.98 \mathrm{E}-09$ & 4.69 & 6.25 & 9.38 \\
\hline & \multicolumn{4}{|c|}{ Number of GPCs Dropped While Loading } & \multicolumn{3}{|c|}{$\begin{array}{l}\text { Amount of total fractures } \\
\text { available for reaction }\end{array}$} \\
\hline & 1 in 100 & 1 in 250 & 1 in 500 & 1 in 1,000 & $\begin{array}{l}2 / 3 \\
\text { available }\end{array}$ & $\begin{array}{l}3 / 4 \\
\text { available }\end{array}$ & $\begin{array}{l}\text { all } \\
\text { available }\end{array}$ \\
\hline & \multicolumn{4}{|c|}{ Probability of waste package loading } & \multicolumn{3}{|c|}{$\begin{array}{c}\text { Fracture factor associated with } \\
\text { available fractures }\end{array}$} \\
\hline $\begin{array}{l}\text { WPs containing } 0 \\
\text { dropped GPCs }\end{array}$ & $9.51 \mathrm{E}-01$ & $9.80 \mathrm{E}-01$ & $9.90 \mathrm{E}-01$ & 9.95E-01 & 1 & 1 & 1 \\
\hline $\begin{array}{l}\text { WPs containing } 1 \\
\text { dropped GPC }\end{array}$ & 4.80E-02 & 1.97E-02 & 9.92E-03 & 4.98E-03 & 4.17 & 4.69 & 6.25 \\
\hline $\begin{array}{l}\text { WPs containing } 2 \\
\text { dropped GPCs }\end{array}$ & $9.70 \mathrm{E}-04$ & $1.58 \mathrm{E}-04$ & $3.98 \mathrm{E}-05$ & 9.97E-06 & 8.33 & 9.38 & 12.50 \\
\hline $\begin{array}{l}\text { WPs containing } 3 \\
\text { dropped GPCs }\end{array}$ & $9.80 \mathrm{E}-06$ & $6.35 \mathrm{E}-07$ & 7.97E-08 & $9.98 \mathrm{E}-09$ & 12.50 & 14.06 & 18.75 \\
\hline
\end{tabular}

NOTE: GPC = Glass Pour Canister, WP = Waste Package.

Table 7-5. True Fracture Factors for High-Level Waste Glass in One Damaged Glass Pour Canister

\begin{tabular}{|c|c|c|c|}
\hline $\begin{array}{c}\text { Total Fractures } \\
\text { Available }\end{array}$ & Fracture Factor & $\begin{array}{c}\text { Total Fractures } \\
\text { Available }\end{array}$ & Fracture Factor \\
\hline $1 / 4$ & 7.81 & $2 / 3$ & 20.83 \\
\hline $1 / 3$ & 10.42 & $3 / 4$ & 23.44 \\
\hline $1 / 2$ & 15.63 & All & 31.25 \\
\hline
\end{tabular}

\subsection{YUCCA MOUNTAIN REVIEW PLAN ACCEPTANCE CRITERIA}

Yucca Mountain Review Plan, Final Report (NRC 2003 [DIRS 163274]) contains Acceptance Criteria that are intended to establish the basis for the review of the material contained in the License Application.

Technical Work Plan for: Regulatory Integration Modeling and Analysis of the Waste Form and Waste Package (BSC 2004 [DIRS 171583], Table 3-1 indicates Acceptance Criteria for this analysis report. The Acceptance Criteria are based on the requirements mentioned in Project 
Requirements Document (Canori and Leitner 2003 [DIRS 166275] and Yucca Mountain Review Plan, Final Report (NRC 2003 [DIR 163274]).

\section{System Description and Demonstration of Multiple Barriers (NRC 2003 [DIRS 163274], Section 2.2.1.1.3)}

For the Yucca Mountain Review Plan criterion entitled System Description and Demonstration of Multiple Barriers (NRC 2003 [DIRS 163274], Section 2.2.1.1.3; Canori and Leitner 2003 [DIRS 166275], PRD-002/T-014, PRD-002/T-016), the acceptance criteria AC1, AC2, and AC3 do not apply because the purpose of this report is not to describe the performance of engineered barriers for the TSPA-LA. Instead, the analysis provides simple statistics on aqueous corrosion rates of steels and alloys.

\section{Degradation of Engineered Barriers (NRC 2003 [DIRS 163274], Section 2.2.1.3.1.3)}

For the Yucca Mountain Review Plan criterion entitled Degradation of Engineered Barriers (NRC 2003 [DIRS 163274], Section 2.2.1.3.1.3; Canori and Leitner 2003 [DIRS 166275], PRD-002/T-015), the acceptance criteria AC1, AC2, AC3, AC4, and AC5 do not apply because the purpose of this report is not to describe deterioration or degradation of engineered barriers for TSPA-LA. Instead, the analysis provides simple statistics on aqueous corrosion rates of steels and alloys. Description of the degradation or deterioration of engineered barriers is covered by other analysis and model reports. 


\section{INTENTIONALLY LEFT BLANK}




\section{INPUTS AND REFERENCES}

\subsection{DOCUMENTS CITED}

164907 Ailor, W.H., Jr. 1969. "A Review of Aluminum Corrosion in Tap Waters.” Journal of Hydronautics, 3, (3), 105-114. Reston, Virginia: American Institute of Aeronautics and Astronautics. TIC: 252903.

162265 Alexander, A.L.; Southwell, C.R.; and Forgeson, B.W. 1961. "Corrosion of Metals in Tropical Environments.” Corrosion, 17, 97-104. Houston, Texas: National Association of Corrosion Engineers. TIC: 223352.

159379 Aziz, P.M. 1956. "Application of the Statistical Theory of Extreme Values to the Analysis of Maximum Pit Depth Data for Aluminum." Corrosion, 12, (10), 35-46. Houston, Texas: National Association of Corrosion Engineers. TIC: 241560.

131480 Bauccio, M., ed. 1993. ASM Metals Reference Book. 3rd Edition. 4th Printing 1999. Volume 1. Materials Park, Ohio: ASM International. TIC: 240701.

106164 Baxter, R.G. 1988. Defense Waste Processing Facility Wasteform and Canister Description. DP-1606, Rev. 2. Aiken, South Carolina: E.I. du Pont de Nemours \& Company, Savannah River Plant. TIC: 8704.

159341 Beavers, J.A. and Durr, C.L. 1991. Immersion Studies on Candidate Container Alloys for the Tuff Repository. NUREG/CR-5598. Washington, D.C.: U.S. Nuclear Regulatory Commission. TIC: 209162.

159339 Beavers, J.A.; Thompson, N.G.; and Durr, C.L. 1992. Pitting, Galvanic, and LongTerm Corrosion Studies on Candidate Container Alloys for the Tuff Repository. NUREG/CR-5709. Washington, D.C.: Nuclear Regulatory Commission. TIC: 212042.

164908 Boatner, L.A. and Sales, B.C. 1988. "Monazite." Chapter 8 of Radioactive Waste Forms for the Future. Lutze, W. and Ewing, R.C., eds. New York, New York: NorthHolland. TIC: 103525.

163699 Bomberger, H.B.; Cambourelis, P.J.; and Hutchinson, G.E. 1954. "Corrosion Properties of Titanium in Marine Environments." Journal of the Electrochemical Society, 101, (9), 442-447. New York, New York: Electrochemical Society. TIC: 254366.

100883 Brasher, D.M. and Mercer, A.D. 1968. "Comparative Study of Factors Influencing the Action of Corrosion Inhibitors for Mild Steel in Neutral Solution, I. Sodium Benzoate.” British Corrosion Journal, 3, 120-129. London, England: British Joint Corrosion Group. TIC: 236434. 
159345 Briant, C.L.; Mulford, R.A.; and Hall, E.L. 1982. "Sensitization of Austenitic Stainless Steels, I. Controlled Purity Alloys." Corrosion, 38, (9), 468-477. Houston, Texas: National Association of Corrosion Engineers. TIC: 225507.

168467 Bryson, J.H.; Preban, A.G.; Coburn, S.K.; Kim, Y-W.; and Lee, H.H. 1987. "Corrosion of Carbon Steels." In Corrosion, Volume 13, Pages 509-530 of Formerly 9th Edition, Metals Handbook. 9th Edition. Metals Park, Ohio: ASM International. TIC: 209807.

156356 BSC (Bechtel SAIC Company) 2001. Degradation of Stainless Steel Structural Material. ANL-EBS-MD-000007 REV 00 ICN 01. Las Vegas, Nevada: Bechtel SAIC Company. ACC: MOL.20010713.0048.

155859 BSC 2001. EBS Incoming Water and Gas Composition Abstraction Calculations for Different Drift Temperature Environments. CAL-EBS-PA-000013 REV 00. Las Vegas, Nevada: Bechtel SAIC Company. ACC: MOL.20011214.0126.

157640 BSC 2001. EQ6 Calculation of Source Terms for DOE Codisposal Waste Packages (Shippingport LWBR, N-Reactor, Melt and Dilute, and Fort St. Vrain). CAL-EDCMD-000017 REV 00. Las Vegas, Nevada: Bechtel SAIC Company. ACC: MOL.20020114.0316.

156183 BSC 2001. Seepage Grout Interactions Model Calculations. CAL-EBS-PA-000014 REV 00. Las Vegas, Nevada: Bechtel SAIC Company. ACC: MOL.20011212.0254.

158828 BSC 2002. EQ6 Calculation for Chemical Degradation of 21 PWR CSNF Waste Packages. CAL-UDC-MD-000002 REV A. Las Vegas, Nevada: Bechtel SAIC Company. ACC: MOL.20020601.0195.

166834 BSC 2003. General Corrosion and Localized Corrosion of Waste Package Outer Barrier. ANL-EBS-MD-000003 REV 01, with 2 errata. Las Vegas, Nevada: Bechtel SAIC Company. ACC: DOC.20030916.0010; DOC.20031222.0002; DOC.20031222.0001.

169110 BSC 2003. PWR Assembly End-Effect Reactivity Evaluation. CAL-UDC-NU-000006 REV 00, with 1 errata. Las Vegas, Nevada: Bechtel SAIC Company.

ACC: MOL.20010412.0158; DOC.20031014.0007.

169107 BSC (Bechtel SAIC Company) 2003. EQ6 Calculation for Chemical Degradation of Enrico Fermi Codisposal Waste Packages: Effects of Updated Design and Rates. CAL-EDC-MD-000015 REV 00 [Errata 001]. Las Vegas, Nevada: Bechtel SAIC Company. ACC: MOL.20020102.0190; DOC.20031014.0012.

171319 BSC (Bechtel SAIC Company) 2004. 21 PWR A, B, D \& E Fuel Plates. 000-MW0DSU0-01201-000-00B. Las Vegas, Nevada: Bechtel SAIC Company. ACC: ENG.20040803.0004. 
171320 BSC (Bechtel SAIC Company) 2004. 21 PWR C Fuel Plate. 000-MW0-DSU001301-000-00B. Las Vegas, Nevada: M\&O. ACC: ENG.20040804.0001.

169220 BSC (Bechtel SAIC Company) 2004. D\&E / PA/C IED Interlocking Drip Shield and Emplacement Pallet. 800-IED-WIS0-00401-000-00D. Las Vegas, Nevada: Bechtel SAIC Company. ACC: ENG.20040503.0018.

169987 BSC (Bechtel SAIC Company) 2004. CSNF Waste Form Degradation: Summary Abstraction. ANL-EBS-MD-000015 REV 02. Las Vegas, Nevada: Bechtel SAIC Company. ACC: DOC.20040908.0001.

169480 BSC 2004. D\&E / PA/C IED Typical Waste Package Components Assembly. 800IED-WIS0-00201-000-00E. Las Vegas, Nevada: Bechtel SAIC Company. ACC: ENG.20040517.0007.

169472 BSC (Bechtel SAIC Company) 2004. D\&E/PA/C IED Typical Waste Package Components Assembly. 800-IED-WIS0-00202-000-00C. Las Vegas, Nevada: Bechtel SAIC Company. ACC: ENG.20040517.0008.

167619 BSC 2004. Defense HLW Glass Degradation Model. ANL-EBS-MD-000016 REV 01 ICN 01. Las Vegas, Nevada: Bechtel SAIC Company. ACC: DOC.20040223.0006.

166862 BSC 2004. Design \& Engineering, 2-MCO/2-DHLW Waste Package Configuration. 000-MW0-DS00-00301-000-00A. Las Vegas, Nevada: Bechtel SAIC Company. ACC: ENG.20040120.0018.

166861 BSC 2004. Design \& Engineering, 5 DHLW/DOE SNF - Long Waste Package Configuration. 000-MW0-DS00-00201-000-00A. Las Vegas, Nevada: Bechtel SAIC Company. ACC: ENG.20040120.0022.

166860 BSC 2004. Design \& Engineering, 5 DHLW/DOE SNF - Short Waste Package Configuration. 000-MW0-DS00-00101-000-00A. Las Vegas, Nevada: Bechtel SAIC Company. ACC: ENG.20040121.0001.

168848 BSC 2004. Drift-Scale Coupled Processes (DST and THC Seepage) Models. MDLNBS-HS-000001 REV 02, with 2 errata. Las Vegas, Nevada: Bechtel SAIC Company. ACC: DOC.20030804.0004; DOC.20040219.0002; DOC.20040405.0005.

167618 BSC (Bechtel SAIC Company) 2004. DSNF and Other Waste Form Degradation Abstraction. ANL-WIS-MD-000004 REV 03. Las Vegas, Nevada: Bechtel SAIC Company.

169845 BSC (Bechtel SAIC Company) 2004. General Corrosion and Localized Corrosion of the Drip Shield. ANL-EBS-MD-000004 REV 02. Las Vegas, Nevada: Bechtel SAIC Company. ACC: DOC.20040921.0002. 
169847 BSC (Bechtel SAIC Company) 2004. Hydrogen-Induced Cracking of the Drip Shield. ANL-EBS-MD-000006 REV 02. Las Vegas, Nevada: Bechtel SAIC Company. ACC: DOC.20040909.0004.

167621 BSC 2004. In-Package Chemistry Abstraction. ANL-EBS-MD-000037, Rev. 03. Las Vegas, Nevada: Bechtel SAIC Company.

168361 BSC 2004. Q-List. 000-30R-MGR0-00500-000-000 REV 00. Las Vegas, Nevada: Bechtel SAIC Company. ACC: ENG.20040721.0007.

171583 BSC 2004. Technical Work Plan For: Regulatory Integration Modeling and Analysis of the Waste Form and Waste Package. TWP-WIS-MD-000009, REV 00, ICN 01. Las Vegas, Nevada: Bechtel SAIC Company. ACC: DOC.20040910.0001.

159368 Butler, F.E. 1963. Corrosion Studies on 304 Stainless Steel Containing One Percent Boron-10. RFP-307. Washington, D.C.: U.S. Atomic Energy Commission. TIC: 252108.

166275 Canori, G.F. and Leitner, M.M. 2003. Project Requirements Document. TER-MGRMD-000001 REV 02. Las Vegas, Nevada: Bechtel SAIC Company. ACC: DOC.20031222.0006.

159369 Cole, H.S. 1976. Corrosion of Stainless Steel Type 304 Alloyed with Boron or Gadolinium by Plant Process Solutions Containing $\mathrm{HNO}_{3}$ and $\mathrm{HF}$. ICP-1097. Idaho Falls, Idaho: Idaho National Engineering Laboratory. TIC: 252106.

100362 CRWMS M\&O 1998. "Waste Form Degradation, Radionuclide Mobilization, and Transport Through the Engineered Barrier System." Chapter 6 of Total System Performance Assessment-Viability Assessment (TSPA-VA) Analyses Technical Basis Document. B00000000-01717-4301-00006 REV 01. Las Vegas, Nevada: CRWMS M\&O. ACC: MOL.19981008.0006.

127351 CRWMS M\&O 1999. EQ6 Calculation for Chemical Degradation of Shippingport PWR (HEU Oxide) Spent Nuclear Fuel Waste Packages. CAL-EDC-MD-000002 REV 00. Las Vegas, Nevada: CRWMS M\&O. ACC: MOL.19991220.0322.

160320 D'Agostino, R.B. and Stephens, M.A., eds. 1986. Goodness-Of-Fit Techniques. Statistics, Textbooks and Monographs Volume 68. New York, New York: Marcel Dekker. TIC: 253256.

154395 Danko, J.C. 1987. "Corrosion in the Nuclear Power Industry." In Corrosion, Volume 13, Pages 927-984 of Metals Handbook. 9th Edition. Metals Park, Ohio: ASM International. TIC: 209807.

162971 Davison, R.M.; DeBold, T.; and Johnson, M.J. 1987. "Corrosion of Stainless Steels." In Corrosion, Volume 13, Pages 547-565 of ASM Handbook. Formerly 9th Edition, Metals Handbook. Materials Park, Ohio: ASM International. TIC: 240704. 
140225 DOE (U.S. Department of Energy) 1999. Design Specification. Volume 1 of Preliminary Design Specification for Department of Energy Standardized Spent Nuclear Fuel Canisters. DOE/SNF/REP-011, Rev. 3. Washington, D.C.: U.S. Department of Energy, Office of Spent Fuel Management and Special Projects. TIC: 246602.

104110 DOE (U.S. Department of Energy) 1999. Fermi (U-Mo) Fuel Characteristics for Disposal Criticality Analysis. DOE/SNF/REP-035, Rev. 0. Washington, D.C.: U.S. Department of Energy. TIC: 242461.

105007 DOE (U.S. Department of Energy) 1999. Shippingport LWBR (Th/U Oxide) Fuel Characteristics for Disposal Criticality Analysis. DOE/SNF/REP-051, Rev. 0. Washington, D.C.: U.S. Department of Energy, Office of Environmental Management. TIC: 245631.

104940 DOE (U.S. Department of Energy) 1999. Shippingport PWR (HEU Oxide) Fuel Characteristics for Disposal Criticality Analysis. DOE/SNF/REP-040, Rev. 0. Washington, D.C.: U.S. Department of Energy. TIC: 243528.

103891 DOE (U.S. Department of Energy) 1999. TRIGA (UZrH) Fuel Characteristics for Disposal Criticality Analysis. DOE/SNF/REP-048, Rev. 0. Washington, D.C.: U.S. Department of Energy. TIC: 244162.

150095 DOE (U.S. Department of Energy) 2000. N Reactor (U-Metal) Fuel Characteristics for Disposal Criticality Analysis. DOE/SNF/REP-056, Rev. 0. Washington, D.C.: U.S. Department of Energy, Office of Environmental Management. TIC: 247956.

158405 DOE (U.S. Department of Energy) 2002. DOE Spent Nuclear Fuel Information in Support of TSPA-SR. DOE/SNF/REP-047, Rev. 2. Idaho Falls, Idaho: U.S. Department of Energy, Idaho Operations Office. TIC: 252089.

168434 DOE (U.S. Department of Energy) 2004. Interim Report on the Corrosion Performance of a Neutron Absorbing Ni-Cr-Mo-Gd Alloy. DOE/SNF/REP-086, Rev. 0. Idaho Falls, Idaho: U.S. Department of Energy, Idaho Operations Office. ACC: DOC.20040412.0001.

171539 DOE (U.S. Department of Energy) 2004. Quality Assurance Requirements and Description. DOE/RW-0333P, Rev. 16. Washington, D.C.: U.S. Department of Energy, Office of Civilian Radioactive Waste Management.

ACC: DOC.20040907.0002.

159367 EPRI (Electric Power Research Institute) 1986. Behavior of High-Density Spent-Fuel Storage Racks. EPRI NP-4724. Palo Alto, California: Electric Power Research Institute. TIC: 252124.

159343 Forgeson, B.W.; Southwell, C.R.; Alexander, A.L.; Mundt, H.W.; and Thompson, L.J. 1958. "Corrosion of Metals in Tropical Environments, (Part 1-Five Non- 
Ferrous Metals and a Structural Steel)." Corrosion, 14, (2), 73t-81t. Houston, Texas: National Association of Corrosion Engineers. TIC: 223373.

159344 Fox, M.J. and McCright, R.D. 1983. An Overview of Low Temperature Sensitization. UCRL-15619. Livermore, California: Lawrence Livermore National Laboratory. ACC: HQS.19880517.2438.

118106 Gdowski, G. E. 1998. Long-Term Corrosion Studies. Activity Plan E-20-50, Rev. 4. Livermore, California: Lawrence Livermore National Laboratory. ACC: MOL.19980810.0274.

100860 Gdowski, G.E. and Bullen, D.B. 1988. Oxidation and Corrosion. Volume 2 of Survey Of Degradation Modes of Candidate Materials for High-Level Radioactive-Waste Disposal Containers. UCID-21362. Livermore, California: Lawrence Livermore National Laboratory. ACC: MOL.19980715.0384.

164946 Ghali, E. 2000. "Aluminum and Aluminum Alloys." Chapter 40 of Uhlig's Corrosion Handbook. Revie, R.W., ed. 2nd Edition. New York, New York: John Wiley \& Sons. TIC: 248360.

159340 Glass, R.S.; Overturf, G.E.; Garrison, R.E.; and McCright, R.D. 1984. Electrochemical Determination of the Corrosion Behavior of Candidate Alloys Proposed for Containment of High Level Nuclear Waste in Tuff. UCID-20174. Livermore, California: Lawrence Livermore National Laboratory. ACC: NNA.19870506.0142.

105021 Glass, R.S.; Overturf, G.E.; Van Konynenburg, R.A.; and McCright, R.D. 1986. "Gamma Radiation Effects on Corrosion-I. Electrochemical Mechanisms for the Aqueous Corrosion Processes of Austenitic Stainless Steels Relevant to Nuclear Waste Disposal in Tuff." Corrosion Science, 26, (8), 577-590. Oxford, Great Britain: Pergamon. TIC: 226179.

164911 Gurwell, W.E. 1981. A Survey of Matrix Materials for Solidified Radioactive HighLevel Waste. PNL-3512. Richland, Washington: Pacific Northwest Laboratory. TIC: 229672.

100455 Hillner, E.; Franklin, D.G.; and Smee, J.D. 1998. The Corrosion of Zircaloy-Clad Fuel Assemblies in a Geologic Repository Environment. WAPD-T-3173. West Mifflin, Pennsylvania: Bettis Atomic Power Laboratory. TIC: 237127.

150403 Hollingsworth, E.H. and Hunsicker, H.Y. 1987. "Corrosion of Aluminum and Aluminum Alloys." In Corrosion, Volume 13, Pages 583-609 of Metals Handbook. 9th Edition. Metals Park, Ohio: ASM International. TIC: 209807.

159334 Hudson, J.C. and Stanners, J.F. 1955. "The Corrosion Resistance of Low-Alloy Steels." Journal of the Iron and Steel Institute, 180, 271-284. London, England: Iron and Steel Institute. TIC: 223378. 
130835 Inco Alloys International. 1988. Product Handbook. Huntington, West Virginia: Inco Alloys International. TIC: 239397.

158820 INEEL (Idaho National Engineering and Environmental Laboratory) 2002. FFTF (MOX) Fuel Characteristics for Disposal Criticality Analysis. DOE/SNF/REP-032, Rev. 1. Idaho Falls, Idaho: U.S. Department of Energy, Idaho National Operations Office. TIC: 252933.

164912 Jorgensen, P.J.; Wadsworth, M.E.; and Cutler, I.B. 1959. “Oxidation of Silicon Carbide." Journal of the American Ceramic Society, 42, (12), 613-616. Easton, Pennsylvania: American Ceramic Society. TIC: 253889.

159385 Kain, R.M.; Tuthill, A.H.; and Hoxie, E.C. 1984. "The Resistance of Types 304 And 316 Stainless Steels to Crevice Corrosion in Natural Waters." Journal of Materials for Energy Systems, 5, (4), 205-211. Metals Park, Ohio: American Society for Metals. TIC: 252968.

134327 Kügler, A. 1997. Sheet and Plate for Nuclear Engineering, Böhler Neutronit A976. Houston, Texas: Böhler Bleche GmbH. TIC: 246410.

164914 Lansdowne Chemicals [n.d.]. Safety Data Sheet, Gadolinium Phosphate. Oxford, England: Lansdowne Chemicals. TIC: 253970.

159337 Larrabee, C.P. 1953. "Corrosion Resistance of High-Strength Low-Alloy Steels as Influenced by Composition and Environment." Corrosion, 9, 259-271. Houston, Texas: National Association of Corrosion Engineers. TIC: 223386.

130543 Lewis, C.F., ed. 1990. Materials Engineering, Materials Selector 1991. Pages 105, 188, and 189. Cleveland, Ohio: Penton Publishing. TIC: 239718.

160832 Lide, D.R., ed. 2002. CRC Handbook of Chemistry and Physics. 83rd Edition. Boca Raton, Florida: CRC Press. TIC: 253582.

164916 Lotts, A.L.; Bond, W.D.; Forsberg, C.W.; Glass, R.W.; Harrington, F.E.; Michaels, G.E.; Notz, K.J.; and Wymer, R.G. 1992. Options for Treating High-Temperature Gas-Cooled Reactor Fuel for Repository Disposal. ORNL/TM-12027. Oak Ridge, Tennessee: Oak Ridge National Laboratory. TIC: 206808.

164942 Matsushima, I. 2000. “Carbon Steel—Corrosion by Seawater.” Chapter 32 of Uhlig's Corrosion Handbook. 2nd Edition. Revie, R.W., ed. New York, New York: John Wiley \& Sons. TIC: 248360.

164944 Matsushima, I. 2000. “Carbon Steel—Corrosion in Fresh Waters.” Chapter 31 of Uhlig's Corrosion Handbook. 2nd Edition. Revie, R.W., ed. New York, New York: John Wiley \& Sons. TIC: 248360.

114637 McCright, R.D. 1998. Corrosion Data and Modeling, Update for Viability Assessment. Volume 3 of Engineered Materials Characterization Report. UCRL-ID- 
119564, Rev. 1.1. Livermore, California: Lawrence Livermore National Laboratory. ACC: MOL.19980806.0177.

159336 McCright, R.D.; Halsey, W.G.; and Van Konynenburg, R.A. 1987. Progress Report on the Results of Testing Advanced Conceptual Design Metal Barrier Materials Under Relevant Environmental Conditions for a Tuff Repository. UCID-21044. Livermore, California: Lawrence Livermore National Laboratory. ACC: HQX.19880201.0016.

164920 Morgan, W.C. 1981. Graphite Matrix Materials for Nuclear Waste Isolation. PNL3556. Richland, Washington: Pacific Northwest Laboratory. TIC: 229659.

163274 NRC (U.S. Nuclear Regulatory Commission) 2003. Yucca Mountain Review Plan, Final Report. NUREG-1804, Rev. 2. Washington, D.C.: U.S. Nuclear Regulatory Commission, Office of Nuclear Material Safety and Safeguards. TIC: 254568.

155502 Opila, E.J. 1999. "Variation of the Oxidation Rate of Silicon Carbide with WaterVapor Pressure." Journal of the American Ceramic Society, 82, (3), 625-636. Westerville, Ohio: American Ceramic Society. TIC: 250063.

167978 Paige, B.E. 1969. Description of Test Reactor Fuel Elements and Associated Behavior in Reprocessing. CI-1152. Idaho Falls, Idaho: U.S. Atomic Energy Commission, Idaho Operations Office. ACC: MOL.20040303.0031.

103896 Parrington, J.R.; Knox, H.D.; Breneman, S.L.; Baum, E.M.; and Feiner, F. 1996. Nuclides and Isotopes, Chart of the Nuclides. 15th Edition. San Jose, California: General Electric Company and KAPL, Inc. TIC: 233705.

111044 Perez, J.M., Jr. and Westsik, J.H., Jr. 1981. "Effects of Cracks on Glass Leaching." Nuclear and Chemical Waste Management, 2, 165-168. New York, New York: Pergamon Press. TIC: 246072.

149395 Propp, W.A. 1998. Graphite Oxidation Thermodynamics/Reactions. DOE/SNF/REP018, Rev. 0. Idaho Falls, Idaho: U.S. Department of Energy. TIC: 247663.

155635 Punatar, M.K. 2001. Summary Report of Commercial Reactor Criticality Data for Crystal River Unit 3. TDR-UDC-NU-000001 REV 02. Las Vegas, Nevada: Bechtel SAIC Company. ACC: MOL.20010702.0087.

101084 Rechard, R.P., ed. 1995. Methodology and Results. Volume 2 of Performance Assessment of the Direct Disposal in Unsaturated Tuff of Spent Nuclear Fuel and High-Level Waste Owned by U.S. Department of Energy. SAND94-2563/2. Albuquerque, New Mexico: Sandia National Laboratories. TIC: 237102.

100417 Rothman, A.J. 1984. Potential Corrosion and Degradation Mechanisms of Zircaloy Cladding on Spent Nuclear Fuel in a Tuff Repository. UCID-20172. Livermore, California: Lawrence Livermore National Laboratory. ACC: NNA.19870903.0039. 
159371 Satyanarayana Gupta, D.V. 1981. "Corrosion Behavior of 1040 Carbon Steel. I. Effect of $\mathrm{pH}$ and Sulfide Ion Concentrations in Aqueous Neutral and Alkaline Solutions at Room Temperature." Corrosion, 37, (11), 611-616. Houston, Texas: National Association of Corrosion Engineers. TIC: 252626.

164921 Sawyer, D.W. and Brown, R.H. 1947. "Resistance of Aluminum Alloys to Fresh Waters." Corrosion, 3, 443-457. Houston, Texas: National Association of Corrosion Engineers. TIC: 252901.

159335 Scarberry, R.C.; Graver, D.L.; and Stephens, C.D. 1967. “Alloying for Corrosion Control, Properties and Benefits of Alloy Materials." Materials Protection, 6, 54-57. Houston, Texas: National Association of Corrosion Engineers. TIC: 251196.

164036 Sedriks, A.J. 1996. Corrosion of Stainless Steels. 2nd Edition. Corrosion Monograph Series. New York, New York: John Wiley \& Sons. TIC: 245121.

164923 Sedriks, A.J. 1982. "Corrosion Resistance of Austenitic Fe-Cr-Ni-Mo Alloys in Marine Environments." International Metals Reviews, 27, (6), 321-353. Metals Park, Ohio: American Society for Metals. TIC: 251201.

164925 Shreir, L.L.; Jarman, R.A.; and Burstein, G.T., eds. 1998. Corrosion Control. Volume 2 of Corrosion. 3rd Edition. Woburn, Massachusetts: Butterworth-Heinemann. TIC: 244694.

100891 Shreir, L.L.; Jarman, R.A.; and Burstein, G.T., eds. 1998. Metal/Environment Reactions. Volume 1 of Corrosion. 3rd Edition. Woburn, Massachusetts: Butterworth-Heinemann. TIC: 244694.

159375 Smith, H.D. 1987. The Influence of Copper on Zircaloy Spent Fuel Cladding Degradation Under a Potential Tuff Repository Condition. UCRL-15993. Livermore, California: Lawrence Livermore National Laboratory. ACC: MOL.19971016.0036.

102089 Smith, P.K. and Baxter, C.A. 1981. Fracture During Cooling of Cast Borosilicate Glass Containing Nuclear Wastes. DP-1602. Aiken, South Carolina: E.I. Du Pont de Nemours \& Company, Savannah River Laboratory. TIC: 238536.

103441 Smith, R.J.; Loomis, G.W.; and Deltete, C. P. 1992. Borated Stainless Steel Application in Spent-Fuel Storage Racks. TR-100784. Palo Alto, California: Electric Power Research Institute. TIC: 225730.

102088 Smith, T.H. and Ross, W.A. 1975. Impact Testing of Vitreous Simulated High-Level Waste in Canisters. BNWL-1903. Richland, Washington: Battelle Pacific Northwest Laboratories. TIC: 238924.

100927 Southwell, C.R.; Bultman, J.D.; and Alexander, A.L. 1976. "Corrosion of Metals in Tropical Environments - Final Report of 16-Year Exposures.” Materials Performance, 15, (7), 9-25. Houston, Texas: National Association of Corrosion Engineers. TIC: 224022. 
154726 Taylor, L.L. 2001. Fort Saint Vrain HTGR (Th/U Carbide) Fuel Characteristics for Disposal Criticality Analysis. DOE/SNF/REP-060, Rev. 0. Washington, D.C.: U.S. Department of Energy, Office of Environmental Management. TIC: 249783.

101231 Uziemblo, N.H. and Smith, H.D. 1989. An Investigation of the Influence of Fluoride on the Corrosion of Zircaloy-4: Initial Report. PNL-6859. Draft. Richland, Washington: Pacific Northwest Laboratory. ACC: MOL.19980326.0369.

100948 Van Konynenburg, R.A.; Curtis, P.G.; and Summers, T.S.E. 1998. Scoping Corrosion Tests on Candidate Waste Package Basket Materials for the Yucca Mountain Project. UCRL-ID-130386. Livermore, California: Lawrence Livermore National Laboratory. ACC: MOL.19980727.0385.

164948 Wallén, B. and Olsson, J. 1977. “Corrosion Resistance in Aqueous Media.” Chapter 16 of Handbook of Stainless Steels. Peckner, D. and Bernstein, I.M., eds. New York, New York: McGraw-Hill. TIC: 219638.

164934 Wheatfall, W.L. 1967. "Metal Corrosion in Deep-Ocean Environments." Naval Engineers Journal, 79, (4), 611-618. Washington, D.C.: American Society of Naval Engineers. TIC: 224024.

100097 Wolery, T.J. and Daveler, S.A. 1992. EQ6, A Computer Program for Reaction Path Modeling of Aqueous Geochemical Systems: Theoretical Manual, User's Guide, and Related Documentation (Version 7.0). UCRL-MA-110662 PT IV. Livermore, California: Lawrence Livermore National Laboratory. ACC: MOL.19980701.0459.

165063 Yau, T.L. and Webster, R.T. 1987. “Corrosion of Zirconium and Hafnium.” In Corrosion, Volume 13, Pages 707-721 of Metals Handbook. 9th Edition. Metals Park, Ohio: ASM International. TIC: 209807.

164938 Zhang, X.G. 2000. "Galvanic Corrosion.” Chapter 8 of Uhlig's Corrosion Handbook. 2nd Edition. Revie, R.W, ed. New York, New York: John Wiley \& Sons.

TIC: 248360.

\subsection{CODES, STANDARDS, REGULATIONS, AND PROCEDURES}

15660510 CFR 63. Energy: Disposal of High-Level Radioactive Wastes in a Geologic Repository at Yucca Mountain, Nevada. Readily available.

10047548 FR 28194. 10 CFR Part 60 Disposal of High-Level Radioactive Wastes in Geologic Repositories Technical Criteria. Readily available.

AP-2.14Q, Rev. 3, ICN 0. Document Review. Washington, D.C.: U.S. Department of Energy, Office of Civilian Radioactive Waste Management.

ACC: DOC.20030827.0018. 
AP-6.1Q, Rev. 7, ICN 4. Document Control. Washington, D.C.: U.S. Department of Energy, Office of Civilian Radioactive Waste Management.

ACC: DOC.20040915.0008.

AP-17.1Q, Rev. 3, ICN 3. Records Management. Washington, D.C.: U.S. Department of Energy, Office of Civilian Radioactive Waste Management. ACC: DOC.20040915.0009.

AP-SIII.9Q, Rev. 1 ICN 7. Scientific Analyses. Washington, D.C.: U.S. Department of Energy, Office of Civilian Radioactive Waste Management.

ACC: DOC.20040920.0001.

AP-SV.1Q, Rev. 1, ICN 1. Control of the Electronic Management of Information. Washington, D.C.: U.S. Department of Energy, Office of Civilian Radioactive Waste Management. ACC: DOC.20040308.0001.

165003 ASTM A 240/A 240M-03b. 2003. Standard Specification for Chromium and Chromium-Nickel Stainless Steel Plate, Sheet, and Strip for Pressure Vessels and for General Applications. West Conshohocken, Pennsylvania: American Society for Testing and Materials. TIC: 254845.

165006 ASTM A 276-03. 2003. Standard Specification for Stainless Steel Bars and Shapes. West Conshohocken, Pennsylvania: American Society for Testing and Materials. TIC: 254842.

162723 ASTM A 516/A 516M-01. 2001. Standard Specification for Pressure Vessel Plates, Carbon Steel, for Moderate- and Lower-Temperature Service. West Conshohocken, Pennsylvania: American Society for Testing and Materials. TIC: 253997.

154062 ASTM A 887-89 (Reapproved 2000). 2000. Standard Specification for Borated Stainless Steel Plate, Sheet, and Strip for Nuclear Application. West Conshohocken, Pennsylvania: American Society for Testing and Materials. TIC: 249544.

144744 ASTM B 209-96. 1996. Standard Specification for Aluminum and Aluminum-Alloy Sheet and Plate. West Conshohocken, Pennsylvania: American Society for Testing and Materials. TIC: 247078.

162726 ASTM B 265-02. 2002. Standard Specification for Titanium and Titanium Alloy Strip, Sheet, and Plate. West Conshohocken, Pennsylvania: American Society for Testing and Materials. TIC: 254000.

168403 ASTM B 932-04. 2004. Standard Specification for Low-Carbon Nickel-ChromiumMolybdenum-Gadolinium Alloy Plate, Sheet, and Strip. West Conshohocken, Pennsylvania: American Society for Testing and Materials. TIC: 255846.

103515 ASTM G 1-90 (Reapproved 1999). 1999. Standard Practice for Preparing, Cleaning, and Evaluating Corrosion Test Specimens. West Conshohocken, Pennsylvania: American Society for Testing and Materials. TIC: 238771. 
LP-SI.11Q-BSC, Rev. 0, ICN 1. Software Management. Washington, D.C.: U.S. Department of Energy, Office of Civilian Radioactive Waste Management.

ACC: DOC.20041005.0008.

\subsection{SOURCE DATA, LISTED BY DATA TRACKING NUMBER}

169583 LL030410012251.056. LTCTF Corrosion Rate Calculations for 2 1/2 - Year Exposed Titanium Alloy GR7 Specimens Cleaned Under TIP-CM-51. Submittal date: 07/16/2003.

151029 MO0006J13WTRCM.000. Recommended Mean Values of Major Constituents in J-13 Well Water. Submittal date: 06/07/2000.

155964 MO0109RIB00049.001. Waste Package Material Properties: Neutron Absorbing Materials. Submittal date: 09/17/2001.

\subsection{OUTPUT DATA, LISTED BY DATA TRACKING NUMBER}

MO0409SPAACRWP.000. Aqueous Corrosion Rates for Non-Waste Form Waste Package Materials. Submittal date: 09/16/2004.

MO0404SPAHLWGF.001. HLW Glass Fracture Factor Probabilities for a Single Waste Package. Submittal Date: 04/22/04. 


\section{APPENDIX I}

\section{LISTING OF FILES ON CD}

Directory of $d: \backslash$

$\begin{array}{ll}09 / 14 / 2004 & 10: 51 \mathrm{a} \\ 03 / 08 / 2004 & 04: 29 \mathrm{p} \\ 09 / 04 / 2004 & 11: 45 \mathrm{a} \\ \text { A110y_.x1s } & \\ 03 / 11 / 2004 & 09: 15 \mathrm{a} \\ 04 / 01 / 2004 & 04: 12 \mathrm{p} \\ 04 / 20 / 2004 & 09: 34 \mathrm{a} \\ 09 / 14 / 2004 & 02: 19 \mathrm{p} \\ 09 / 15 / 2004 & 09: 20 \mathrm{a} \\ 08 / 13 / 2004 & 02: 35 \mathrm{p} \\ 08 / 10 / 2004 & 10: 37 \mathrm{a} \\ 08 / 10 / 2004 & 10: 52 \mathrm{a} \\ 10 / 04 / 2004 & 11: 20 \mathrm{a}\end{array}$

Total Files Listed:

12 File (s)

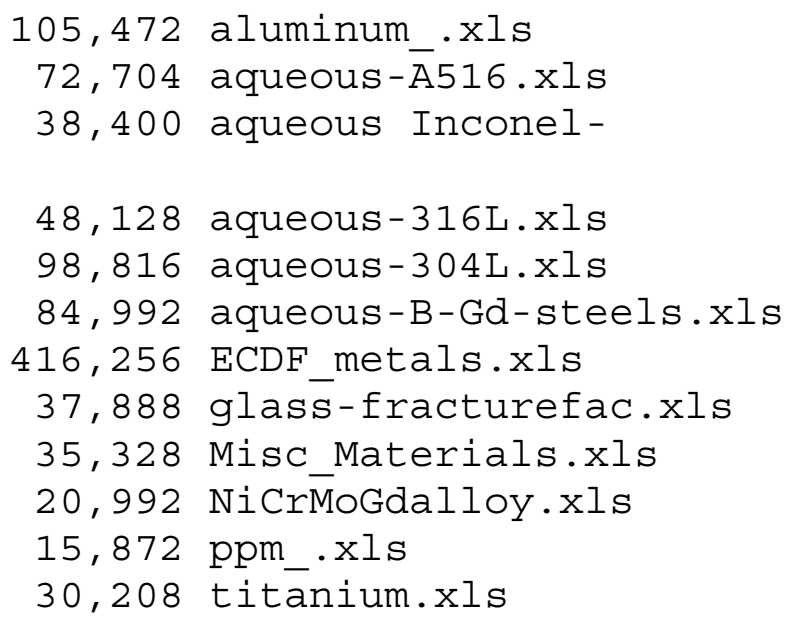

1,005,056 bytes 


\section{INTENTIONALLY LEFT BLANK}




\section{APPENDIX II}

\section{Attached CD-ROM}




\section{INTENTIONALLY LEFT BLANK}




\section{APPENDIX III \\ Qualification of Data That Supports Aqueous Corrosion Rates for Waste Packages Materials (ANL-DSD-MD-000001)}

\section{III.1 PURPOSE}

This analysis relies on data that may not have been collected under an approved quality assurance program that meets the requirements of 10 CFR Part 63, Subpart G [DIRS 156605] or its predecessor, 10 CFR Part 60 (48 FR 28194 [DIRS 100475]). This appendix qualifies the data this analysis uses as direct input.

\section{III.2 METHODS AND CRITERIA}

This appendix qualifies direct input data used in this analysis for intended use per AP-SIII.9Q, Scientific Analyses. The qualification process indicates (as required in AP-SIII.9Q, Section 5.2.11) that the qualification shall address "the extent to which the data demonstrate the properties of interest." This is indicated in this appendix by the heading "Demonstrate properties of interest." The qualification process in this appendix also considers at least one of the following factors:

- Reliability of data source

- Qualifications of personnel or organizations generating the data

- Prior use of the data

- Availability of corroborating data.

\section{III.3 QUALIFICATION OF DATA}

Table III-1 lists all sources whose data is to be qualified for intended use in this appendix. The table designates the attributes or criteria used in conjunction with the corroborating data and discussion of data collection methodology to qualify the information. 
Table III-1. Sources of Data to be Qualified and Criteria Used to Qualify the Data

\begin{tabular}{|c|c|c|}
\hline Data & Source & Criteria \\
\hline Corrosion Rates of Aluminum Alloys & Ailor 1969 [DIRS 164907] & Corroborating Data \\
\hline $\begin{array}{l}\text { Corrosion Rates of Stainless Steel } \\
\text { Types } 302,316 \text {, and } 321\end{array}$ & Alexander et al. 1961 [DIRS 162265] & $\begin{array}{l}\text { Corroborating Data } \\
\text { Personnel/organization } \\
\text { qualifications } \\
\text { Reliability of data source }\end{array}$ \\
\hline Corrosion rate of monazite & Boatner and Sales 1988 [DIRS 164908] & Corroborating Data \\
\hline $\begin{array}{l}\text { Corrosion Rates of Stainless Steel } \\
\text { Types } 302 \text { and } 316\end{array}$ & Bomberger et al. 1954 [DIRS 163699] & Corroborating Data \\
\hline $\begin{array}{l}\text { Corrosion rates of borated and Gd- } \\
\text { alloyed Stainless Steel Types } 304\end{array}$ & Cole et al. 1976 [DIRS 159369] & Corroborating Data \\
\hline Corrosion Rates of Alloy N06464 & DOE 2004 [DIRS 168434] & $\begin{array}{l}\text { Personnel/organization } \\
\text { qualifications } \\
\text { Reliability of data source }\end{array}$ \\
\hline Corrosion Rates of Aluminum Alloys & Forgeson et al. 1958 [DIRS 159343] & $\begin{array}{l}\text { Corroborating Data } \\
\text { Personnel/organization } \\
\text { qualifications } \\
\text { Reliability of data source }\end{array}$ \\
\hline $\begin{array}{l}\text { Corrosion Rates of Stainless Steel } \\
\text { Types } 302,316 \text {, and } 321\end{array}$ & Glass et al. 1984 [DIRS 159340] & Corroborating Data \\
\hline Oxidation rate of graphite in water & Gurwell 1981 [DIRS 164911] & Corroborating Data \\
\hline General Corrosion of Zircaloys & Hillner et al. 1998 [DIRS 100455] & Corroborating Data \\
\hline Oxidation rate of graphite & Lewis 1990 [DIRS 130543] & Corroborating Data \\
\hline Oxidation rate of graphite & Lotts et al. 1992 [DIRS 164916] & Corroborating Data \\
\hline $\begin{array}{l}\text { Corrosion Rates of Stainless Steel } \\
\text { Types } 304 \text { and } 316\end{array}$ & McCright et al. 1987 [DIRS 159336] & $\begin{array}{l}\text { Corroborating Data } \\
\text { Personnel/organization } \\
\text { qualifications } \\
\text { Reliability of data source }\end{array}$ \\
\hline $\begin{array}{l}\text { Corrosion rates of Inconel alloys and } \\
\text { Carbon Steel Types A516 and A27 }\end{array}$ & McCright 1998 [DIRS 114637] & $\begin{array}{l}\text { Corroborating Data } \\
\text { Personnel/organization } \\
\text { qualifications } \\
\text { Reliability of data source } \\
\end{array}$ \\
\hline Oxidation rate of graphite & Morgan 1981 [DIRS 164920] & Corroborating Data \\
\hline Oxidation rate of $\mathrm{SiC}$ & Opila 1999 [DIRS 155502] & $\begin{array}{l}\text { Corroborating Data } \\
\text { Personnel/organization } \\
\text { qualifications } \\
\text { Reliability of data source }\end{array}$ \\
\hline Oxidation rate of graphite in air & Propp 1998 [DIRS 149395] & Corroborating Data \\
\hline General Corrosion of Zircaloys & Rothman 1984 [DIRS 100417] & Corroborating Data \\
\hline Corrosion Rates of Aluminum Alloys & Sawyer and Brown 1947 [DIRS 164921] & $\begin{array}{l}\text { Corroborating DataReliability of } \\
\text { data source }\end{array}$ \\
\hline Oxidation rate of graphite & Shreir et al. 1998 [DIRS 164925] & Corroborating Data \\
\hline $\begin{array}{l}\text { Corrosion Rates of Aluminum Alloys } \\
\text { Corrosion Rates of Stainless Steel } \\
\text { Types } 302,316 \text {, and } 321 \text { and } \\
\text { detection limit }\end{array}$ & Southwell et al. 1976 [DIRS 100927] & $\begin{array}{l}\text { Corroborating Data } \\
\text { Personnel/organization } \\
\text { qualifications } \\
\text { Reliability of data source }\end{array}$ \\
\hline $\begin{array}{l}\text { Corrosion Rates of } 304 \text { Stainless } \\
\text { Steel }\end{array}$ & Wheatfall 1967 [DIRS 164934] & Corroborating Data \\
\hline
\end{tabular}




\section{III.3.1 Qualification of Data in Ailor, W.H. 1969 [DIRS 164907]}

Demonstrate properties of interest: The tests were carried out specifically to assess the corrosion of aluminum in tap waters (dilute waters for the purposes of this analysis).

Corroborating Data: An authoritative source, Hollingsworth and Hunsiker (1987 [DIRS 150403]) corroborate the use of this source, as does Ghali (2000 [DIRS 164946]). Both references provide a synopsis of corrosion behavior of aluminum in different environments. Southwell et al. (1976 [DIRS 100927]) provides quantitative corroboration by showing corrosion rates in good agreement with those of Ailor (1969 [DIRS 164907].

\section{III.3.2 Qualification of Data in Alexander, A.L.; Southwell, C.R.; and Forgeson, B.W. 1961 [DIRS 162265]}

Demonstrate properties of interest: Tests carried out on materials of specific interest in waste package corrosion (Stainless Steel Types 302, 316, and 321) in water types of interest (dilute and salt and concentrated waters)

Corroborating Data: Quantitative corroboration is provided by two authoritative sources, Beavers and Durr 1991 [DIRS 159341] and Beavers et al. 1992 [DIRS 159339], which provide corrosion data on Stainless Steel Type 304L (similar to Stainless Steel Type 302) and Stainless Steel Type 316L (Stainless Steel Type 316 with slightly less carbon content). Glass et al. (1984 [DIRS 159340]) provides corrosion data on Stainless Steel Type 321. Qualitative data used for corroboration through description of stainless steel corrosion behavior in aqueous media include Hudson and Stanners (1955 [DIRS 159334]), Scarberry (1967 [DIRS 159335]), Wallen and Olson (1977 [DIRS 164948]), and Davison et al. (1987 [DIRS 162971]), which is included in ASM International's Metals Handbook, considered an authoritative source.

Reliability of data source and Personnel/organization qualifications: The data source is a publication by the NACE. The NACE is currently the largest provider of information on corrosion and is relied upon globally by industries and governments for standards for materials preservation and corrosion control information. All publications from the NACE are rigorously peer reviewed before publication. The authors are recognized scientists in the field of materials corrosion. They have years of experience in experimental design and data collection in corrosion studies and are eminently qualified to conduct these studies.

\section{III.3.3 Qualification of Data in Boatner, L.A. and Sales, B.C. 1988 [DIRS 164908]}

Demonstrate properties of interest: The purpose of the assessment done by Boatner and Sales (1988 [DIRS 164908]) was to examine the possibility of using monazite as a medium for nuclear waste disposal. This also included an assessment of the pure material, which is directly applicable to this analysis.

Corroborating Data: Boatner and Sales (1988 [DIRS 164908]) present a degradation rate of monazite containing 20\% Savannah River waste at less than $0.001 \mathrm{~g} / \mathrm{m}^{2} /$ day (approximately $0.12 \mu \mathrm{m} / \mathrm{yr}$, presented as an upper bound in Section 6.3.3) and continue by stating that pure monazite would have much greater corrosion resistance. This is corroborated by Lansdowne 
(n.d. [DIRS 164914]) (an authoritative source and manufacturer of $\mathrm{GdPO}_{4}$ material), which states that the material is "insoluble in water."

\section{III.3.4 Qualification of Data in Bomberger, H.B.; Cambourelis, P.J.; and Hutchinson, G.E. 1954 [DIRS 163699]}

Demonstrate properties of interest: Tests carried out on materials of specific interest in waste package corrosion (Stainless Steel Types 302 and 316L) in water types of interest (salt and concentrated waters)

Corroborating Data: Quantitative corroboration is provided by two authoritative sources, Beavers and Durr 1991 [DIRS 159341] and Beavers et al. 1992 [DIRS 159339], which provide corrosion data on Stainless Steel Type 304L (similar to Stainless Steel Type 302) and Stainless Steel Type 316L (Stainless Steel Type 316 with slightly less carbon content). Qualitative data used for corroboration through description of stainless steel corrosion in aqueous media include data by Hudson and Stanners (1955 [DIRS 159334]), Scarberry (1967 [DIRS 159335]), Wallen and Olson (1977 [DIRS 164948]), and Davison et al. (1987 [DIRS 162971]), which is included in ASM International's Metals Handbook, considered an authoritative source.

\section{III.3.5 Qualification of Data in Cole, H.S. 1976 [DIRS 159369]}

Demonstrate properties of interest: Tests carried out on neutron absorbing materials of specific interest in waste package corrosion (boron-alloyed Stainless Steel Type 304) in water types of interest ("fresh" and "harsh" waters).

Corroborating Data: Cole (1976 [DIRS 159369]) presents the degradation of Stainless Steel Type 304 alloyed with boron. These rates indicate that with increasing boron content, the corrosion rate increases significantly. This is corroborated by Van Konynenburg et al. (1998 [DIRS 100948]); who show increase in corrosion with the addition of boron, and Smith et al. (1992 [DIRS 103441]) (a report published by the Electrical Power Research Institute (EPRI), an authoritative source) who show that as boron content of the stainless steel increases, the corrosion rate of the material also increases. Because of their proximity to one another on the periodic table, boron can be likened to carbon. It is well known that increased carbon content of stainless steels and heat-treating induces carbide formation. These carbides then affect the stability of the oxide film allowing the corrosion rate to increase.

\section{III.3.6 Qualification of Data in DOE 2004 [DIRS 168434]}

Demonstrate properties of interest: Tests carried out on Alloy N06464 to qualify material for acceptance in the Yucca Mountain Repository as a neutron absorbing material.

Reliability of data source and Personnel/organization qualifications: The data source was peer reviewed. Preparation and cleaning of test coupons as well as practice for laboratory immersion corrosion testing of metals were conducted following ASTM standards (as stated in Section 3.4 of DOE 2004 [DIRS 168434]). One of the purposes of these tests was to qualify the material for ASTM code qualification. The material was accepted as an ASTM standard and presented in ASTM B 932-04 (2004 [DIRS 168403]). 


\section{III.3.7 Qualification of Data in Forgeson, B.W.; Southwell, C.R.; Alexander, A.L.; Mundt, H.W.; and Thompson, L.J. 1958 [DIRS 159343]}

Demonstrate properties of interest: Tests carried out on materials of specific interest in waste package corrosion (aluminum alloys) in water types of interest (dilute, salt and concentrated waters).

Corroborating Data: An authoritative source, Hollingsworth and Hunsiker (1987 [DIRS 150403]) corroborate the use of this source, as does Ghali (2000 [DIRS 164946]). Both references provide a synopsis of corrosion behavior of aluminum in different environments. Ailor (1969 [DIRS 164907]) provides quantitative corroboration by showing corrosion rates in good agreement with those by Forgeson et al. (1958 [DIRS 159343]).

Reliability of data source and Personnel/organization qualifications: The data source is a publication by the NACE. The NACE is currently the largest provider of information on corrosion and is relied upon globally by industries and governments for standards for materials preservation and corrosion control information. All publications from the NACE are rigorously peer reviewed before publication. The authors are recognized scientists in the field of materials corrosion. They have years of experience in experimental design and data collection in corrosion studies and are eminently qualified to conduct these studies.

\section{III.3.8 Qualification of Data in Glass, R.S.; Overturf, G.E.; Garrison, R.E.; and McCright, R.D. 1984 [DIRS 159340]}

Demonstrate properties of interest: Tests carried out on materials of specific interest in waste package corrosion (Stainless Steel Types 304L, 316L, and 321) in water types of interest (dilute "freshwater").

Corroborating Data: Quantitative corroboration is provided by two authoritative sources, Beavers and Durr 1991 [DIRS 159341] and Beavers et al. 1992 [DIRS 159339], which provide corrosion data on Stainless Steel Types 304L and 316L (Stainless Steel Type 321 is close in composition to Stainless Steel Type 316L, so it should have similar corrosion properties). Qualitative data used for corroboration through description of stainless steel corrosion in aqueous media include Hudson and Stanners (1955 [DIRS 159334]), Scarberry (1967 [DIRS 159335]), Wallen and Olson (1977 [DIRS 164948]), and Davison et al. 1987 [DIRS 162971], which is included in ASM International's Metals Handbook, considered an authoritative source.

\section{III.3.9 Qualification of Data in Gurwell, W.E. 1981 [DIRS 164911]}

Demonstrate properties of interest: Assessment was carried out to evaluate the feasibility of disposal using matrix waste forms (waste pellets or marbles within matrix material). Part of this assessment included evaluation of the oxidation of graphite.

Corroborating Data: Graphite is known to be highly corrosion resistant in both atmospheric and aqueous conditions. Gurwell (1981 [DIRS 164911]) states that graphite reacts very slowly in deionized water with a corrosion rate at $99^{\circ} \mathrm{C}$ of $10^{-10} \mathrm{~g} / \mathrm{cm}^{2} /$ day (or approximately $1.7 \times 10^{-4} \mu \mathrm{m} / \mathrm{yr}$ ). This rate is slow enough essentially to be considered, noncorrosive to insoluble, especially at lower temperatures. This noncorrosive or insoluble behavior is 
corroborated by experimental data from Lotts et al. (1992 [DIRS 164916]) and Propp (1998 [DIRS 149395]) who report corrosion rates even slower than those reported by Gurwell (1981 [DIRS 164911]). Additionally, Lide (2002 [DIRS 160832], an authoritative source), Morgan (1981 [DIRS 164920]), and Shreir et al. 1998 [DIRS 164925] all report that the material is unreactive or insoluble.

III.3.10 Qualification of Data in Hillner, E.; Franklin, D.G.; and Smee, J.D. 1998 [DIRS 100455]

Demonstrate properties of interest: Tests carried out to assess zircaloy cladding corrosion in long term conditions of geologic repository disposal.

Corroborating Data: Three sources corroborate the behavior of zircaloy as described by Hillner et al. (1998 [DIRS 100455]). Yau and Webster (1987 [DIRS 165063]) (an authoritative source) indicate that zirconium and its alloys hold up well to a number of different corrosive environments. Uziemblo and Smith (1989 [DIRS 101231]) further corroborate this by indicating a corrosion rate of zero in the temperature range of $21.5^{\circ} \mathrm{C}$ to $43^{\circ} \mathrm{C}$. Rothman $(1984$ [DIRS 100417]) indicates that after 10,000 years at $180^{\circ} \mathrm{C}$, the depth of oxidation of zircaloy would be between 4 and $53 \mu \mathrm{m}$. All references corroborate the slow corrosion or oxidation rate of zircaloy presented by Hillner et al. (1998 [DIRS 100455]).

\section{III.3.11 Qualification of Data in Lewis, C.F., ed. 1990 [DIRS 130543]}

Demonstrate properties of interest: gives overview of graphite oxidation. This is directly applicable since carbide fuels will be disoposed inside of their original fuel elements composed of high-grade industrial graphite.

Corroborating Data: Graphite is known to be highly corrosion resistant in both atmospheric and aqueous conditions. Lewis (1990 [DIRS 130543]) states that industrial graphite has "excellent corrosion resistance." This corrosion resistant behavior is corroborated by experimental data from Lotts et al. (1992 [DIRS 164916]), Gurwell (1981 [DIRS 164911]), and Propp (1998 [DIRS 149395]), who record very slow corrosion for graphite (nanometers or less per year depending on temperature). Additionally, Lide (2002 [DIRS 160832], an authoritative source), Morgan (1981 [DIRS 164920]), and Shreir et al. 1998 [DIRS 164925] report that the material is unreactive or insoluble.

\section{III.3.12 Qualification of Data in Lotts, A.L.; Bond, W.D.; Forsberg, C.W.; Glass, R.W.; Harrington, F.E.; Michaels, G.E.; Notz, K.J.; and Wymer, R.G. 1992 [DIRS 164916]}

Demonstrate properties of interest: Assessment was carried out to evaluate the feasibility of disposal options for high-temperature gas-cooled (HTGR) reactor fuels. Part of this assessment included evaluation of the oxidation of graphite (material composing the reactor elements). It was this assessment that was used in this analysis.

Corroborating Data: Graphite is known to be highly corrosion resistant in both atmospheric and aqueous conditions. Lotts et al. (1992 [DIRS 164916]) show that air oxidation of graphite 
produces a corrosion rate of $4.5 \times 10^{-20} \mathrm{~g} / \mathrm{cm}^{2} / \mathrm{sec}$ (or approximately $6.6 \times 10^{-9} \mu \mathrm{m} / \mathrm{yr}$ ) at $100^{\circ} \mathrm{C}$. This rate is slow enough to be considered, essentially, noncorrosive to insoluble, especially at lower temperatures. This noncorrosive or insoluble behavior is corroborated by experimental data from Propp (1998 [DIRS 149395]) and Gurwell (1981 [DIRS 164911]). Additionally, Lide (2002 [DIRS 160832]), an authoritative source, Morgan (1981 [DIRS 164920]), and Shreir et al. 1998 [DIRS 164925] all report that the material is unreactive or insoluble.

\section{III.3.13 Qualification of Data in McCright, R.D.; Halsey, W.G.; and Van Konynenburg, R.A. 1987 [DIRS 159336]}

Demonstrate properties of interest: Tests carried out on materials of specific interest in waste package corrosion (Stainless Steel Types 304L and 316L) in water types of interest (dilute or fresh waters).

Corroborating Data: Quantitative corroboration is provided by two authoritative sources, Beavers and Durr (1991 [DIRS 159341]) and Beavers et al. (1992 [DIRS 159339]), who provide corrosion data on Stainless Steel Types 304L and 316L. Qualitative data used for corroboration through description of stainless steel corrosion in aqueous media include Hudson and Stanners (1955 [DIRS 159334]), Scarberry (1967 [DIRS 159335]), Wallen and Olson 1977 [DIRS 164948]), and Davison et al. (1987 [DIRS 162971], which is included in ASM International's Metals Handbook, considered an authoritative source.

Personnel/organization qualifications and reliability of source: The experiments and analysis were performed at the Lawrence Livermore National Laboratory using good engineering practices. Also used a well accepted methodology and experiments were carried out in a thorough and comprehensive manner. Preparation and cleaning of test coupons as well as practice for laboratory immersion corrosion testing of metals were conducted following ASTM standards (as stated by McCright et al. (1987 [DIRS 159336], p. 22)).

\section{III.3.14 Qualification of Data in McCright, R.D. 1998 [DIRS 114637]}

Demonstrate properties of interest: Experiments carried out specifically to determine the corrosive behavior of candidate materials (Carbon Steel Type A516, Inconel Alloy) proposed for waste packages in a repository setting.

Corroborating Data: Quantitative corroboration is provided by Brasher and Mercer (1968 [DIRS 100883]), who indicate rates of corrosion similar to those presented by McCright (1998 [DIRS 114637]). This reference also shows the strong temperature dependence of corrosion rates that is also demonstrated by McCright (1998 [DIRS 114637]). Qualitative data used for corroboration through description of carbon steel corrosion in aqueous media include those provided by Matsushima (2000 [DIRS 164942]), Matsushima (2000 [DIRS 164944]), Satyanarayana (1981 [DIRS 159371]), and Bryson (1987 [DIRS 168467], which is included in ASM International's Metals Handbook, considered an authoritative source.

Personnel/organization qualifications and reliability of source: The experiments and analysis were performed at the Lawrence Livermore National Laboratory using good engineering practices, a well-accepted methodology, and in a thorough and comprehensive manner. Testing 
and analysis were done to an approved activity plan (Gdowski 1998 [DIRS 118106]) and technical implementation plans (McCright 1998 [DIRS 114637], p. 2.2-11 for more detail). Materials were cleaned in accordance with ASTM Standard G 1-90 [DIRS 103515].

\section{III.3.15 Qualification of Data in Morgan, W.C. 1981 [DIRS 164920]}

Demonstrate properties of interest: Study carried out specifically answer question on the feasibility of using graphite matrix materials in a repository environment.

Corroborating Data: Graphite is known to be highly corrosion resistant in both atmospheric and aqueous conditions. Morgan (1981 [DIRS 164920]) states that graphite is insoluble at "ordinary temperatures." This non-corrosive or insoluble behavior is corroborated by experimental data from Lotts et al. (1992 [DIRS 164916]), Gurwell (1981 [DIRS 164911]), and Propp (1998 [DIRS 149395]), which record very slow corrosion for graphite (nanometers or less per year depending on temperature). Additionally, Lide (2002 [DIRS 160832], an authoritative source) and Shreir et al. (1998 [DIRS 164925]) report the material is unreactive or insoluble.

\section{III.3.16 Qualification of Data in Opila, E.J. 1999 [DIRS 155502]}

Demonstrate properties of interest: Study carried out specifically to answer question on $\mathrm{SiC}$ oxidation in water vapor. Comparisons to oxidation in water at like temperatures were then used to derive the rate required for this analysis.

Personnel/organization qualifications and reliability of source: The experiments and analysis were performed good engineering practices, used a well accepted methodology, and in a thorough and comprehensive manner. Testing included the use of pure material obtained from manufacturer. Equipment used was specifically designed to strictly control and monitor experimental conditions.

Corroborating data: Jorgensen et al. (1959 [DIRS 164912]) corroborate the behavior of SiC at different temperatures and show that the material is extremely durable, even at higher temperatures. The references also show that as the temperature decreases, the corrosion rate decreases significantly. Following this continual decrease, Lide (2002 [DIRS 160832]) indicates that $\mathrm{SiC}$ is insoluble at $25^{\circ} \mathrm{C}$ in water, which is also corroborated by Lotts et al. (1992 [DIRS 164916]) who indicate that the $\mathrm{SiC}$ layer on the TRISO particles will fail only under "unknown mechanisms."

\section{III.3.17 Qualification of Data in Propp, W.A. 1998 [DIRS 149395]}

Demonstrate properties of interest: Study carried out specifically to answer question on graphite oxidation in a repository environment.

Corroborating Data: Graphite is known to be highly corrosion resistant in both atmospheric and aqueous conditions. Propp (1998 [DIRS 149395]) states that air oxidation of graphite produces a corrosion rate of $1 \mathrm{mg} / \mathrm{cm}^{2}$ in 190,000 years (or approximately $2.4 \times 10^{-5} \mu \mathrm{m} / \mathrm{yr}$ ). This rate is slow enough to be considered, essentially, noncorrosive to insoluble, especially at lower temperatures. This noncorrosive or insoluble behavior is corroborated by experimental data from Lotts et al. (1992 [DIRS 164916]) and Gurwell (1981 [DIRS 164911]). Additionally, Lide 
(2002 [DIRS 160832], an authoritative source), Morgan (1981 [DIRS 164920]), and Shreir et al. (1998 [DIRS 164925]) report the material is unreactive or insoluble.

\section{III.3.18 Qualification of Data in Rothman, A.J. 1984 [DIRS 100417]}

Demonstrate properties of interest: Tests carried out to assess zircaloy cladding corrosion in long term conditions of geologic repository disposal.

Corroborating Data: Three sources corroborate the behavior of zircaloy as described by Rothman (1984 [DIRS 100417]). Yau and Webster (1987 [DIRS 165063]) (an authoritative source) indicate that zirconium and its alloys hold up well to a number of different corrosive environments. Uziemblo and Smith (1989 [DIRS 101231]) further corroborate this by indicating a corrosion rate of zero in the temperature range of $21.5^{\circ} \mathrm{C}$ to $43^{\circ} \mathrm{C}$. Hillner et al. (1998 [DIRS 100455]) indicates that after one million years, oxide growth on zircaloy would be about 0.3 mils $(1 \mathrm{mil}=25.4 \mu \mathrm{m})$. All references corroborate the slow corrosion or oxidation rate of zircaloy presented by Rothman (1984 [DIRS 100417]).

\section{III.3.19 Qualification of Data in Sawyer, D.W. and Brown, R.H. 1947 [DIRS 164921]}

Demonstrate properties of interest: study carried out for materials of specific interest in waste package corrosion (aluminum alloys) in water types of interest (dilute or freshwater).

Corroborating Data: An authoritative source, Hollingsworth and Hunsiker (1987 [DIRS 150403]) corroborate the use of this source, as does Ghali (2000 [DIRS 164946]). Both references provide a synopsis of corrosion behavior of aluminum in different environments. Ailor (1969 [DIRS 164907]) provides quantitative corroboration by showing corrosion rates in good agreement with those by Sawyer and Brown (1947 [DIRS 164921]).

Reliability of data source: The data source is a publication by the NACE. The NACE is currently the largest provider of information on corrosion and is relied upon globally by industries and governments for standards for materials preservation and corrosion control information. All publications from the NACE are rigorously peer reviewed before publication.

\section{III.3.20 Qualification of Data in Shreir, L.L.; Jarman, R.A.; and Burstein, G.T., eds 1998 [DIRS 164925]}

Demonstrate properties of interest: Specifically looks at carbonaceous material (Shreir et al. 1998 [DIRS 164925], Section 18.1) and gives properties of durability and corrosion resistance in a number of corrosive environments.

Corroborating Data: Graphite is known to be highly corrosion resistant in both atmospheric and aqueous conditions. Shreir et al. (1998 [DIRS 164925]) state that graphite is generally unreactive at low temperatures and provide a table (18.2) showing that the material is resistant to a great number of highly corrosive environments. This noncorrosive or insoluble behavior is corroborated by experimental data by Lotts et al. (1992 [DIRS 164916]), Gurwell (1981 [DIRS 164911]), and Propp (1998 [DIRS 149395]), which record very slow corrosion for graphite (nanometers or less per year depending on temperature). Additionally, Lide (2002 [DIRS 160832]) (an authoritative source) and Morgan (1981 [DIRS 164920]) report that the material is unreactive or insoluble. 


\section{III.3.21 Qualification of Data in Southwell, C.R.; Bultman, J.D.; and Alexander, A.L. 1976 [DIRS 100927]}

Demonstrate properties of interest: Tests carried out on materials of specific interest in waste package corrosion (Stainless Steel Types 302 and 316 and aluminum alloys) in water types of interest (dilute and salt or concentrated waters).

Corroborating Data: For stainless steel corrosion, quantitative corroboration is provided by two authoritative sources, Beavers and Durr (1991 [DIRS 159341]) and Beavers et al. (1992 [DIRS 159339]), which provide corrosion data on Stainless Steel Types 304L (similar to Stainless Steel Type 302) and 316L (similar in composition to Stainless Steel Type 316). Glass et al. (1984 [DIRS 159340]) provides corrosion data on Stainless Steel Type 321. Qualitative data used for corroboration through description of stainless steel corrosion in aqueous media include Hudson and Stanners (1955 [DIRS 159334]), Scarberry (1967 [DIRS 159335]), Wallen and Olson (1977 [DIRS 164948]), and Davison et al. (1987 [DIRS 162971], which is included in ASM International's Metals Handbook, considered an authoritative source. For aluminum corrosion, an authoritative source, Hollingsworth and Hunsicker (1987 [DIRS 150403]) corroborate the use of this source, as does Ghali (2000 [DIRS 164946]). The references provide a synopsis of corrosion behavior of aluminum in different environments. Ailor (1969 [DIRS 164907]) provides quantitative corroboration for aluminum corrosion and shows corrosion rates in good agreement with those by Southwell et al. (1976 [DIRS 100927]).

Reliability of data source and Personnel/organization qualifications: The data source is a publication by the NACE. The NACE is currently the largest provider of information on corrosion and is relied upon globally by industries and governments for standards for materials preservation and corrosion control information. All publications from the NACE are rigorously peer reviewed before publication. The authors are recognized scientists in the field of materials corrosion. They have years of experience in experimental design and data collection in corrosion studies and are eminently qualified to conduct these studies.

\section{III.3.22 Qualification of Data in Wheatfall, W.L. 1967 [DIRS 164934]}

Demonstrate properties of interest: Tests carried out on materials of specific interest in waste package corrosion (Stainless Steel Type 304) in waters of interest (salt or concentrated waters).

Corroborating Data: Quantitative corroboration is provided by two authoritative sources, Beavers and Durr 1991 [DIRS 159341] and Beavers et al. 1992 [DIRS 159339], which provide corrosion data on Stainless Steel Type 304L (Stainless Steel Type 304 with a slightly lower carbon content). Qualitative data used for corroboration through description of stainless steel corrosion in aqueous media include Hudson and Stanners (1955 [DIRS 159334]), Scarberry (1967 [DIRS 159335]), Wallen and Olson (1977 [DIRS 164948]), and Davison et al. (1987 [DIRS 162971], which is included in ASM International's Metals Handbook, considered an authoritative source.

\section{III.4 CONCLUSION}

In conclusion, the data is qualified for its intended use in this analysis. 